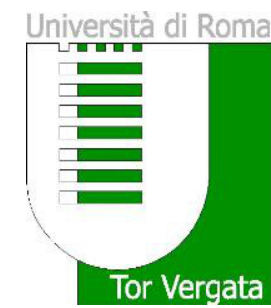

UNIVERSITÀ DEGLI STUDI DI ROMA

"TOR VERGATA"

FACOLTÀ DI SCIENZE MATEMATICHE, FISICHE E NATURALI

TESI DI DOTTORATO DI RICERCA IN FISICA

\title{
Polarization Observables from two-pion and $\rho$ meson photoproduction on polarized HD target at JLab
}

Irene Zonta

Relatore:

Coordinatore:

Prof. Annalisa D'Angelo

Prof. Maurizio De Crescenzi 



\section{Contents}

$\begin{array}{lr}\text { Preface } & 7\end{array}$

1 Introduction and Overview 13

1.1 Motivation for photoproduction experiments . . . . . . . . . . . 13

1.2 Polarization observables: exploiting the spin degree of freedom . . . 23

1.2.1 Another degree of freedom: the Isospin . . . . . . . . . . . 32

1.3 Motivation for this analysis . . . . . . . . . . . . 36

$\begin{array}{lll}2 & \text { The experimental apparatus } & \mathbf{3 7}\end{array}$

2.1 CEBAF: Continuous Electron Beam Accelerator Facility . . . . . . . 39

2.2 Experimental Hall B . . . . . . . . . . . . . . . . . . . . . 40

2.3 Beamline Devices . . . . . . . . . . . . . . . . . . . . . . . . . 42

2.3.1 Beam Position Monitors . . . . . . . . . . . . . . . . 42

2.3.2 Beam Profile Monitors . . . . . . . . . . . . . . . . . 42

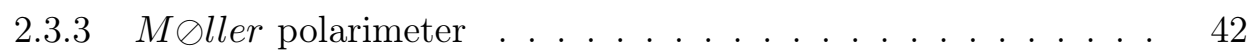

2.3.4 Pair Spectrometer . . . . . . . . . . . . . . . . 43

2.3.5 Total Absorption Shower Counter . . . . . . . . . . . . . 43

2.3.6 Photon Beam Collimation . . . . . . . . . . . . . 47

2.3 .7 The photon tagger . . . . . . . . . . . . . . . 48

2.4 The HD frozen spin polarized target . . . . . . . . . 50

2.5 The CLAS detector . . . . . . . . . . . . . . . . 50

2.5.1 Start counter . . . . . . . . . . . . . . 50

2.5.2 Superconducting Toroidal Magnet . . . . . . . . . . 52

2.5.3 Drift chambers . . . . . . . . . . . . . . . 53

2.5.4 Time-of-flight scintillators . . . . . . . . . . . . 55

2.5.5 Forward electromagnetic calorimeter . . . . . . . . . 55

3 The HD-ice target $\quad 59$

3.1 Introduction . . . . . . . . . . . . . . . . . . . . . . . 59

3.2 HD-ice Target geometry . . . . . . . . . . . . . . . . . . . 60

3.3 HD-ice Target physics . . . . . . . . . . . . . . . . . . . . . . . . 61

3.3.1 HD gas distillation . . . . . . . . . . . . . . . . . 64 
3.4 HD Gas characterization . . . . . . . . . . . . . . . . . . 68

3.4.1 Rotational Raman spectroscopy . . . . . . . . . . . . . 68

3.4.2 Theory of rotational Raman scattering . . . . . . . . . . . . 69

3.4.3 Raman spectroscopy set-up and procedures . . . . . . . . 73

3.5 Raman analysis results . . . . . . . . . . . . . . . 76

3.6 The HD-ice Target equipment: cryostats . . . . . . . . . . . . 83

3.6.1 The Production Dewar . . . . . . . . . . . . . . . . 84

3.6.2 The Transfer Cryostat . . . . . . . . . . . . . . . . . . 84

3.6.3 The Dilution Fridge . . . . . . . . . . . . . . . . 85

3.6 .4 The Storage Dewar . . . . . . . . . . . . . . . . . 87

3.6.5 The In-Beam Cryostat . . . . . . . . . . . . . . . . . 87

3.7 The HD-ice Target production . . . . . . . . . . . . . . . . . . . . 91

3.7.1 Condensing the HD gas . . . . . . . . . . . . . . . . . 91

3.7 .2 Thermal equilibrium calibration . . . . . . . . . . . . 91

3.7.3 Transferring the target to the Dilution Fridge . . . . . . . . . 92

3.7.4 Polarization of the HD-ice Target . . . . . . . . . . . . 92

3.7.5 Measuring the HD-ice Target polarization . . . . . . . . . . 93

3.7.6 Transporting the HD-ice Target to Hall B . . . . . . . . . . 93

3.7.7 Measuring the final HD-ice Target polarization . . . . . . . . 94

3.7 .8 HD gas storage . . . . . . . . . . . . . . . . . 94

$4 \gamma N \rightarrow \pi^{+} \pi^{-} N$ event selection from the g14 data 95

4.1 Running condition . . . . . . . . . . . . . . . . 95

4.2 Organization of the g14 data: collection, calibration and cooking . . 96

4.2.1 The forward electromagnetic calorimeter timing calibration . 97

4.2 .2 Simulation chain . . . . . . . . . . . . . . . . 102

4.3 Data banks and first data skimming . . . . . . . . . . . . . . 103

4.4 Applied corrections to the data . . . . . . . . . . . . . . . 105

4.5 Kinematic Fitting . . . . . . . . . . . . . . . . . . . 105

4.5.1 CLAS tracking parameters . . . . . . . . . . . . 106

4.6 Energy and Momentum Corrections . . . . . . . . . . . . . . . 107

4.6.1 Energy Loss correction . . . . . . . . . . . . . . . . . . 107

4.6.2 Tagger correction . . . . . . . . . . . . . . . . . . . . . . 109

4.6.3 Momentum correction . . . . . . . . . . . . . . . . . . 109

4.6.4 Time-of-flight of neutral particles correction . . . . . . . . . . 112

4.7 Neutron mis-identification . . . . . . . . . . . . . . . . . . . . 114

4.8 Event selection cuts . . . . . . . . . . . . . . . . . . . . . . . . 118

$4.8 .1 \quad$ z-vertex cut . . . . . . . . . . . . . . . . . . . . . . . 118

4.8.2 Incident Photon identification . . . . . . . . . . . . . . 118

4.8.3 Removing accidentals . . . . . . . . . . . . . . . . 120

4.8 .4 Missing mass cut . . . . . . . . . . . . . . . . . . . . . . 122

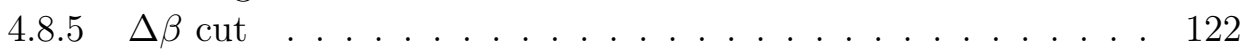


$4.9 \quad$ Final $\pi^{+} \pi^{-} p$ and $\pi^{+} \pi^{-} n$ sample . . . . . . . . . . . . . . . 125

$\begin{array}{llr}5 & \text { Extraction of polarization observables } & 129\end{array}$

5.1 Angles definition and bin choice . . . . . . . . . . . . . . . 129

5.2 Observables for double pion photoproduction on the polarized protons and neutrons . . . . . . . . . . . . . . 130

5.3 Extraction of the Beam Helicity asymmetry $\left(I^{\odot}\right) \ldots \ldots . . \ldots 131$

5.4 Extraction of the Helicity Difference $\left(P_{z}^{\odot}\right) \ldots \ldots \ldots$

5.5 The reaction $\gamma \mathrm{p} \rightarrow \rho^{0} \mathrm{p} \ldots \ldots \ldots \ldots . \ldots \ldots 138$ 5.5.1 The $\rho$ vector meson . . . . . . . . . . . . . . . . . 138

5.6 Invariant Mass distributions . . . . . . . . . . . . . . . . . . . 141

5.7 Background estimation and sPlot method . . . . . . . . . . 153

5.8 Extraction of the Beam-Target asymmetry $(\mathrm{E}) \ldots \ldots \ldots$

$\begin{array}{llr}6 & \text { Conclusions } & 157\end{array}$

$\begin{array}{lc}\text { APPENDICES } & 157\end{array}$

A Least squares fitting with Lagrange multipliers $\quad 159$ 


\section{Preface}

In this section I will give a brief overview of my research experience. The preliminary results discussed in this theses have been carried out with the Nuclear Physics group of the Department of Physics at the University of Rome Tor Vergata, under the supervision of Prof. A. D'Angelo. On October 2010 I won a scholarship for a $\mathrm{PhD}$ position at the University of Rome Tor Vergata; I started my work as a PhD student on November 2010. During the first PhD year I took three exams and attended other three classes especially on theoretical physics to complete my cultural background and expanding my horizon as a experimental physicist.

On March 2012 I joined the CLAS collaboration at the Thomas Jefferson National Laboratory, in Virginia, USA, and became a term member after a probation period of 9 months. As a member I could participate to the g14 data taking, started on November 18th 2011 and finished on May 18th 2012. In that period I was in charge of the timing calibration of the CLAS forward electromagnetic spectrometer.

For the duration of the experiment, I was also responsible of the Raman laboratory located at the University of Rome Tor Vergata, where the Raman measurements were performed. The Raman measurements were crucial for determining the relative concentrations of $\mathrm{H}_{2}$ and $\mathrm{D}_{2}$ in the Hydrogen-Deuteride gas which was used for the target of the g14 experiment.

After the end of the experiment, I started my analysis on the g14 data focusing on the reaction channels $\gamma \mathrm{N} \rightarrow \pi^{+} \pi^{-} \mathrm{N}$ where two charged pions are detected in coincidence with the recoil nucleon (either a proton or a neutron). The final scope of this thesis work was the extraction of two of the polarization observables for double charged pion photoproduction both on proton and neutron target: the beam asymmetry $\left(I^{\odot}\right)$, which arises if the photon beam is circularly polarized, and the helicity difference, $P_{z}^{\odot}$ which arises if the photon beam is circularly polarized and the target is longitudinal polarized.

In the summer 2014 I developed a correction for the neutron time-of-flight for the g14 data, which basically took into account where the neutron hit the electromagnetic calorimeter.

I analyzed the reaction $\gamma \mathrm{N} \rightarrow \rho \mathrm{N}$ and where $\mathrm{N}$ is the proton and the neutron, supporting my analysis also with Monte Carlo simulations.

The photoproduction of the $\rho$ vector meson is not an easy subject to study, especially 
at the photon energy range of the g14 experiment $(\sim 1-2 \mathrm{GeV})$, since the signal contribution cannot be easily disentangled form the background coming from the reactions $\gamma p \rightarrow \pi^{+} \Delta^{0}, \gamma p \rightarrow \pi^{-} \Delta^{++}$and the non-resonant photoproduction of two charged pions, $\gamma p \rightarrow \pi^{+} \pi^{-} p$. As a first approach, is it possible to disentangle the reaction $\gamma \mathrm{N} \rightarrow \rho$ from the background by imposing values of the moment transfer to the recoil proton smaller than $0.5 \mathrm{GeV} / \mathrm{c}$. I also studied and tried to apply different sophisticated statistical methods, the QValue and the sPlot in order to give a correct evaluation of the remaining background. This permitted to extract the double polarization observable asymmetry $\mathrm{E}$, for the proton target, which is measured for the first time.

The last month has been almost entirely dedicated to write this thesis.

I'm co-authors of the following scientific works:

During this period I have been involved in the following scientific works:

1. "Experimental study of the P11(1440) and D13(1520) resonances from the CLAS data on ep $\rightarrow e^{\prime} \pi^{+} \pi^{-} p^{\prime \prime}$

V. I. Mokeev and the CLAS Collaboration

Phys. Rev. C 86, 035203 (2012)

2. "Measurement of Exclusive $\pi^{0}$ Electroproduction Structure Functions and their Relationship to Transversity GPDs"

I.Bedlinskly and the CLAS Collaboration

Phys. Rev. Lett. 109, 112001 (2012)

3. "Deep exclusive $\pi^{+}$electroproduction off the proton at CLAS"

K. Park and the CLAS Collaboration

Eur. Phys. J. A 49 (2013) 16

4. "Near-threshold neutral pion electroproduction at high momentum transfer and generalized form factors"

P. Khetarpal and the CLAS Collaboration

Phys. Rev. C 87, 045205 (2013)

5. "Measurement of transparency ratios for protons from short-range correlated pairs"

O. Hen and the CLAS Collaboration

Phys. Lett. B 722, 63-68 (2013)

6. "HD gas purification for polarized HDice targets production at Jefferson Lab" C. S. Whisnant et al.

PoS PSTP2013 017

7. "Separated Structure Function for Exclusive $K^{+} \Lambda$ and $K^{+} \Sigma^{0}$ Electroproduction at $5.5 \mathrm{GeV}$ with CLAS" 
D.S. Carman and the CLAS Collaboration

Phys. Rev. C 87, 025204 (2013)

8. "Measurement of the $\Sigma \pi$ photoproduction line shapes near the $\Lambda(1405)$ "

Moriya and the CLAS Collaboration

Phys. Rev. C 87, 035206 (2013)

9. "Transverse Polarization of the $\Sigma^{+}(1189)$ in Photoproduction on a Hydrogen Target in CLAS"

C.S. Nepali and the CLAS Collaboration

Phys. Rev. C 87, 045206 (2013)

10. "Hard Two-Body Photodisintegration of 3He"

I. Pomerantz and the CLAS Collaboration

Phys. Rev. Lett. 110, 242301 (2013)

11. "Cross sections for the $\gamma p \rightarrow K^{*+} \Lambda$ and $\gamma p \rightarrow K^{*+} \Sigma^{0}$ reactions measured at CLAS"

W. Tang and the CLAS Collaboration

Phys. Rev. C 87, 065204 (2013)

12. "Differential photoproduction cross sections of the $\Sigma^{0}(1385), \Lambda(1405)$ and $\Lambda(1520) "$

K. Moriya and the CLAS Collaboration

Phys. Rev. C 88, 045201 (2013)

13. "Demonstration of a novel technique to measure two-photon exchange effects in elastic $e^{ \pm} \mathrm{p}$ scattering"

M. Moteabbed and the CLAS Collaboration

Phys. Rev. C 88, 025210 (2013)

14. "First observation of the $\Lambda(1405)$ line shape in electroproduction"

H. Y. Lu and the CLAS Collaboration

Phys. Rev. C 88, 045202 (2013)

15. "Beam asymmetry $\Sigma$ for $\pi^{+}$and $\pi^{0}$ photoproduction on the proton for photon energies from 1.102 to $1.862 "$

M. Dugger and the CLAS Collaboration

Phys. Rev. C 88, 065203 (2013)

16. "Separated Structure Functions for Exclusive $K^{+} \Lambda$ and $K^{+} \Sigma^{0}$ Electroproduction at $5.5 \mathrm{GeV}$ with CLAS"

D. Carman and the CLAS Collaboration

Phys. Rev. C 87, 025204 (2013) 
17. "Measurement of the free neutron structure function using spectator tagging in inelastic $d\left(e, e^{\prime} p_{s}\right) X$ scattering with CLAS"

S. Tkachenko and the CLAS Collaboration

Phys. Rev. C 89, 045206 (2014)

18. " $\Phi$-meson photoproduction on Hydrogen in the neutral decay mode"

H. Serydaryan and the CLAS Collaboration

Phys. Rev. C 89, 055206 (2014)

19. "Spin and Parity Measurement of the $\Lambda(1405)$ baryon"

K. Moryia and the CLAS Collaboration

Phys. Rev. Lett. 112, 082004 (2014)

20. "Beam-spin Asymmetries from Semi-inclusive Pion Electroproduction"

W. Gohn and the CLAS Collaboration

Phys. Rev. D 89, 072011 (2014)

21. "Near Threshold Neutral Pion Electroproduction at High Momentum Transfers and Generalized Form Factors"

D. Carman and the CLAS Collaboration

Phys. Rev. C 87, 045205 (2014)

22. "Precision measurement of $\mathrm{g} 1$ of the proton and the deuteron with $6 \mathrm{GeV}$ electrons"

Y. Prok and the CLAS Collaboration

Phys. Rev. C. 90, 025212 (2014)

23. "Data analysis techniques, differential cross sections and spin density matrix elements for the reaction $\gamma p \rightarrow \phi p "$

B. Dey and the CLAS Collaboration

Phys. Rev. C. 89, 055208 (2014)

24. "Exclusive $\pi^{0}$ electroproduction at $\mathrm{W}>2 \mathrm{GeV}$ with CLAS"

I. Bedlinskiy

Phys. Rev. C. 90, 039901 (2014)

25. "Momentum sharing in imbalanced Fermi systems"

O. Hen and the CLAS Collaboration

Science 346, 6209 (2014)

26. "Induced polarization of $\Lambda(1116)$ in kaon electroproduction"

M. Gabrielyan and the CLAS Collaboration

Phys. Rev. C. 90, 035202 (2014) 
27. "Strangeness Suppression of $q \bar{q}$ Creation Observed in Exclusive Reactions" M. Mestayer and the CLAS Collaboration Phys. Rev. Lett 113, 152004 (2014)

28. "Beam asymmetry Sigma in eta-prime photoproduction off the proton at the GRAAL experiment" G. Mandaglio and the GRAAL Collaboration EPJ Web Conf 7304006 (2014)

29. "Design and realization of a facility for the characterization of Silicon Avalanche PhotoDiodes"

A. Celentano et al. JINST 9 09, T09002 (2014)

30. "Towards a resolution of the proton from factor problem: new electron and positron scattering data"

D. Adikaram and the CLAS Collaboration

Phys. Rev. Lett. 114, 062003 (2015)

31. "Longitudinal target-spin asymmetries for deeply virtual Compton scattering" E. Seder and the CLAS Collaboration Phys. Rev. Lett 114, 032001 (2015)

32. "Single and double spin asymmetries for deeply virtual Compton scattering measured with CLAS and a longitudinally polarized proton target"

S. Pisano and the CLAS Collaboration Phys. Rev. D 91, 052014 (2015) 


\section{Chapter 1}

\section{Introduction and Overview}

\subsection{Motivation for photoproduction experiments}

Hadrons are composite objects consisting of quarks which carry, in addiction to electric charge, a strong charge or color. The field quanta, or gauge bosons, of the strong interactions are called gluons, zero mass particles, which, like quarks, carry color. The fundamental coupling for the strong interaction is then between quarks and gluons, with a strength proportional to $\sqrt{\alpha_{s}}$ where $\alpha_{s}$ is the strong coupling constant; a quark-quark interaction proceeds via the exchange of a virtual intermediate gluon. In a similar way, the electromagnetic interaction between charged particles is mediated by a massless gauge boson, a photon; the strength of the electromagnetic coupling is proportional to $\sqrt{\alpha_{e m}}$. The analogy with the electromagnetic interactions is not as close as it could appear. Let us see why.

First of all, the fine-structure constants are not actually constants; these quantities are function of the distance because of vacuum polarization, an effect which may be described in analogy with the electrostatic of dielectrics.

If a medium, containing molecules with a permanent electric dipole moment, is introduced into an electric field, that field is reduced within the dielectric by a factor $\epsilon$, being $\epsilon$ the dielectric constant. If the field is due to a pair of charges \pm q placed within the medium, the molecules of the medium will align along the field direction and produce a screening or reduction in the effective value of the two charges, as shown in Figure 1.1.

In proximity of the charges, the effective charge is the bare charge q reduced by the "surface" charge. The electrostatic potential may be written:

$$
V=\frac{1}{4 \pi \epsilon_{0} \epsilon(r)} \frac{q^{2}}{r}
$$

which tells us that over short distances the dielectric constant $\epsilon$ is a function of the distance $r$. For values of $r$ much larger than the molecular diameter $d, \epsilon(r)$ 


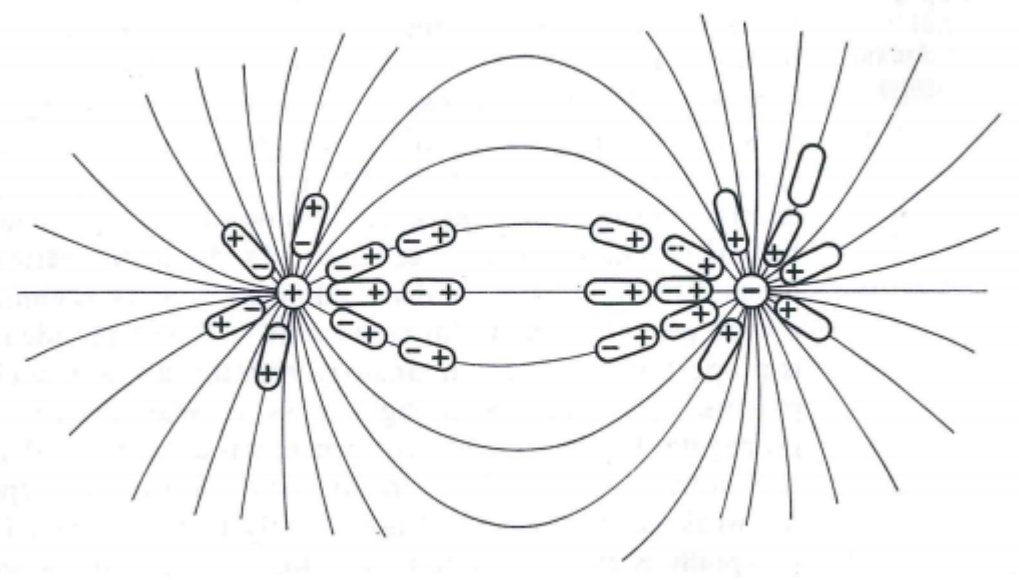

Figure 1.1: Schematic representation of macroscopic charge screening in a dielectric medium [1].

tends to the dielectric constant $\epsilon$, while for $\mathrm{r} \ll \mathrm{d} \epsilon(r)$ tends to 1 . The effective charge $q / \sqrt{\epsilon(r)}$ varies with the distance as shown in Figure 1.2.

For distances small compared with the molecular diameter, the screening effect diminishes; the effective charge increases and approaches the value of the full bare charge q.

Similar screening effect are seen when considering the charge of the electron. According to the Quantum Electrodynamics (QED) an electron can spontaneously emit a photon which may materialize into an electron-positron pair, the electron and positron may emit further photons an so on. The electron is thus surrounded by a fluctuating cloud of virtual electro-positron pairs with radius of the order of the Compton wavelength, $\lambda=\hbar / m c$, as shown in Figure 1.3.

Because of the Coulomb attraction, the positrons will, on average, be closer to the bare electron and the vacuum will be polarized. At distances large compared to the Compton wavelength the effective charge is normally quoted as the charge on the electron $e=1.602 \times 10^{-19}$ coulomb, corresponding to a value for the fine structure $\alpha=e^{2} / 4 \pi \epsilon_{0} \hbar c \sim 1 / 137$.

In analogy with the macroscopic effect described before, as the charge is probed at increasingly shorter distances the effective charge increases, corresponding to a growth of the fine-structure constant, as shown in Figure 1.4.

This effect is rather small, about $1 \%$, but detectable. It must be noted that since the photon carries no charge, there is no direct photon-photon coupling in QED. This leads to what is known as an Abelian field theory.

Vacuum polarization has effect also on the values of the strong charges but in a 


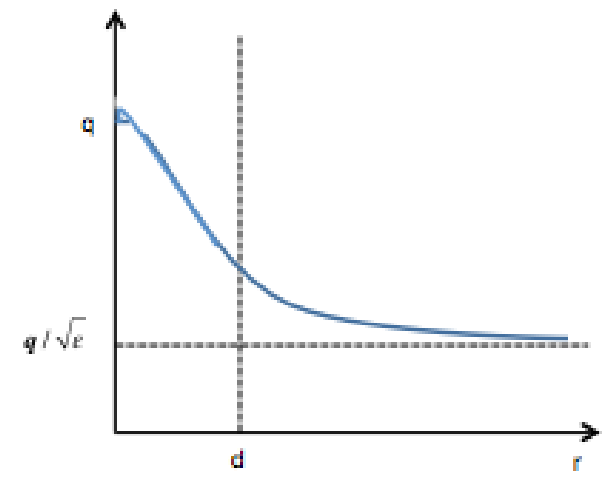

Figure 1.2: Sketch of the effective charge as a function of separation distance $r$; $d$ is the molecular diameter in a general case.

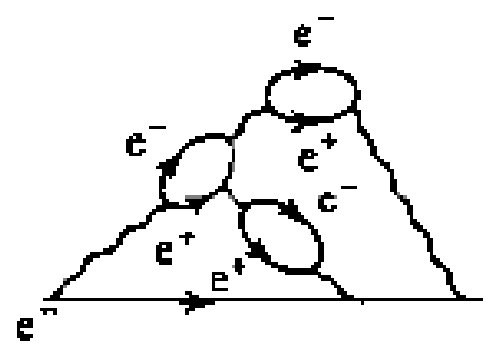

Figure 1.3: Vacuum polarization in QED [1]. 


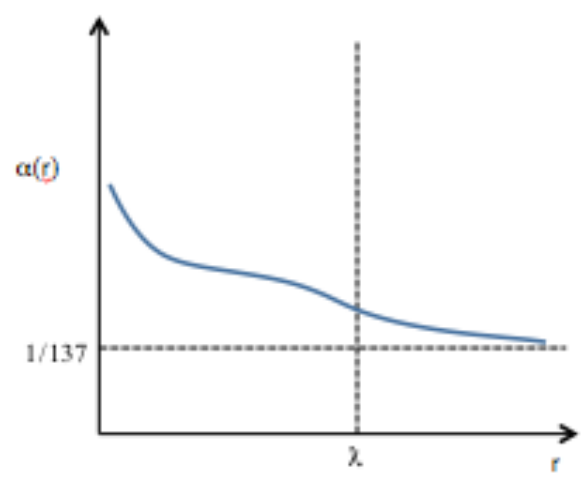

Figure 1.4: Variation of the fine-structure constant $\alpha$ as a function of distance.

crucially different way ${ }^{1}$. The difference lies in the fact that the gluon carries color, unlike the photon which has no electric charge. Now, the gluons can couple together; this self-coupling leads to the so-called non-Abelian field theories.

The bare color charge of a quark is modified by vacuum polarization described in QCD by diagrams like those shown in Figure 1.5.
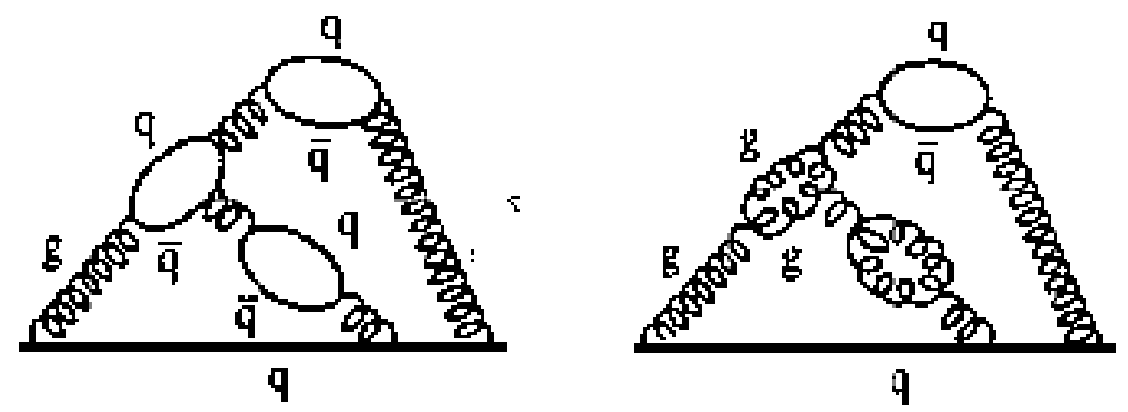

Figure 1.5: Two contributions from the vacuum polarization effect in QCD. Left: a contribution from $g q g$ coupling. Right: a contribution including triple-gluon coupling [1].

If the polarization was described only by diagrams like the one in the left-hand side of Figures 1.5, the behavior of the strong coupling constant $\alpha_{s}$ as a function of the distance would be the same as in the electromagnetic case.

${ }^{1}$ Vacuum polarization has effect also on weak charges. 
It was discovered by Gross, Wilczek and Politzer, that diagrams of the type shown in the right-hand side of Figures 1.5, which include self-coupling vertices, have an antiscreening effect. We can imagine the effect of the gluon self-interaction like if the gluon "tended to carry color away from a quark" or dilute the color charge, so that, in contrast to the electromagnetic case, the color charge decreases as it is probed to shorter and shorter distances. Indeed $\alpha_{s}$ approaches zero as $r \rightarrow 0$. This behavior is called asymptotic freedom, and it implies that at very small distances, which can be achieved using a high energy probe, the quarks behave like free particles. The behavior of $\alpha_{s}$ as a function of the distance $\mathrm{r}$ and the transferred momentum $\mathrm{Q}$ is schematically shown in Figure 1.6 and 1.7, respectively.

One fundamental "practical" consequence of this behavior is that, at high energies, perturbative methods can be used in the evaluation of QCD diagrams, and the success of this approach has been confirmed by several experiments; in the low energy regime, corresponding to the scale typical of the nucleon masses and it excited states $(\sim 1 \mathrm{GeV})$, the perturbative approach is no more valid and a solution to QCD cannot be found.

As the physicist Y. L. Dokshitzar said: "QCD is the strangest of all theories. On one hand it is beyond any doubt the microscopic theory of the hadron world. On the other hand it has a split personality. It embodies both hard and soft physics, but the soft part is the harder".

Another spectacular results of the anti-screening of the strong interaction is the mass origin of the hadrons. According to its quark content (uud triplet), the proton should have a mass of approximately $9.4 \mathrm{MeV}$. In reality, the proton is 100 times heavier. This means that $99 \%$ of the proton mass is generated dynamically by the self-interaction of the gluons which bind the quarks. The proton can be conceived as a bound state of three so-called "constituent quarks" which have a mass one third of the proton mass. A constituent quark is a quark dressed by a cloud of virtual gluons and quarks, from which it acquires its effective mass, as shown in Figure 1.8.

In order to explain properties of hadrons, such as their masses and resonance spectra, a different theoretical approach is needed.

The more recent and promising results in this sense are those coming from Lattice QCD calculations, where the strong interaction theory is formulated on discretized space-time and treated numerically [9]; some groups carried out simulations with pion masses as light as $156 \mathrm{MeV}$, but they mostly focused on states with lower values of angular momentum, as shown in Figures 1.9 [12].

In other calculations carried out using larger pion masses $\left(m_{\pi} \sim 400 \mathrm{MeV}\right)$, a rich spectrum of excited states was obtained, which is shown in Figure 1.10. The lowlying states have the same quantum numbers as the states in models based on three constituent quarks with wave functions based on the irreducible representations of $S U(3) \otimes S U(6)$.

In these approaches, called "constituent quark models", where the valence quarks 


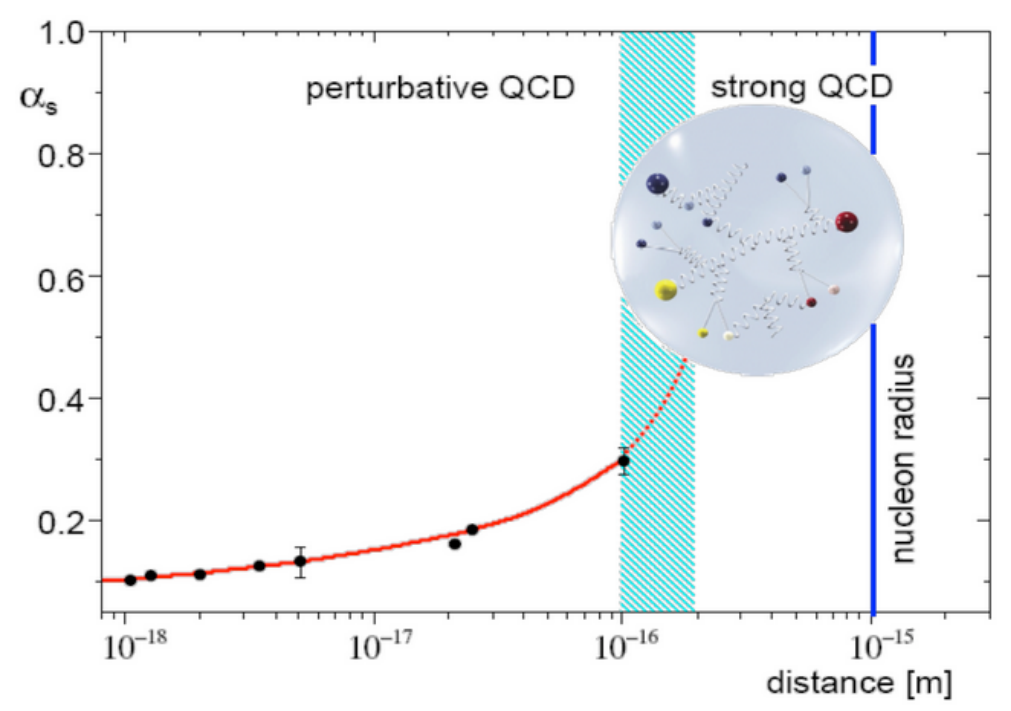

Figure 1.6: The strong coupling constant, $\alpha_{s}$, as a function of the distance scale. Towards larger distances, QCD becomes non-perturbative, and gives rise to spectacular phenomena such as the generation of hadron masses and quark confinement [2].

are replaced by constituent quarks, excited states of hadrons emerge from different arrangements of the constituent quarks in an effective potential. These models are able to explain the observed nucleon and $\Delta$-resonance spectrum, but also predict a number of excited state that are not observed experimentally, the so-called missing resonances.

An alternative approach, based on different effective degrees of freedom, is the quarkdiquark model, where the internal baryon dynamics is described by single constituent quarks joined by a tightly bound constituent quark pair. This approach effectively reduces the number of degrees of freedom, leading to fewer predicted excited states in the predicted baryon spectra.

However, inconsistencies with the observed excited spectra remain: the higher part of the excitation spectra in quark-diquark models still predicts resonances that are not observed.

The question of whether this mismatch between experiment and theoretical prediction is due to a bias in experiment or to a "wrong choice" of the internal degrees of freedom considered by the current models remains open and it could be solved only through joint efforts of experimentalists and theorists.

From the experimental side already in 1960 Koniug and Isgur in their work titled "Where have all the resonances gone?" [14] tried to address the question suggesting that the missing states may be weakly coupled to channels where the pion is in the 


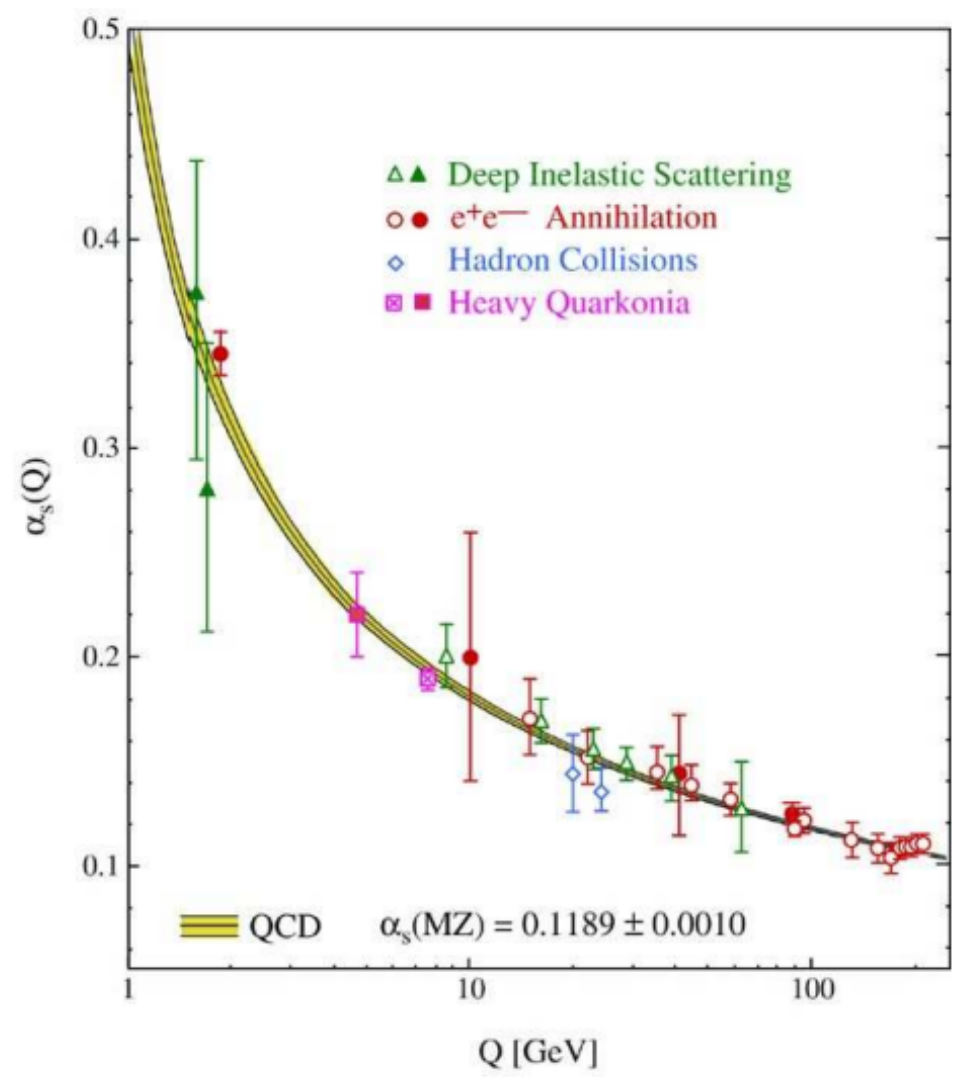

Figure 1.7: The strong coupling constant, $\alpha_{s}$, as a function of the momentum transfer. It decreases with increasing momentum transfer. It's value is most precisely known from $e^{+} e^{-}$scattering near the mass of the $Z_{0}$ boson $\left(91.187 \mathrm{GeV} / \mathrm{c}^{2}\right)[6]$. 


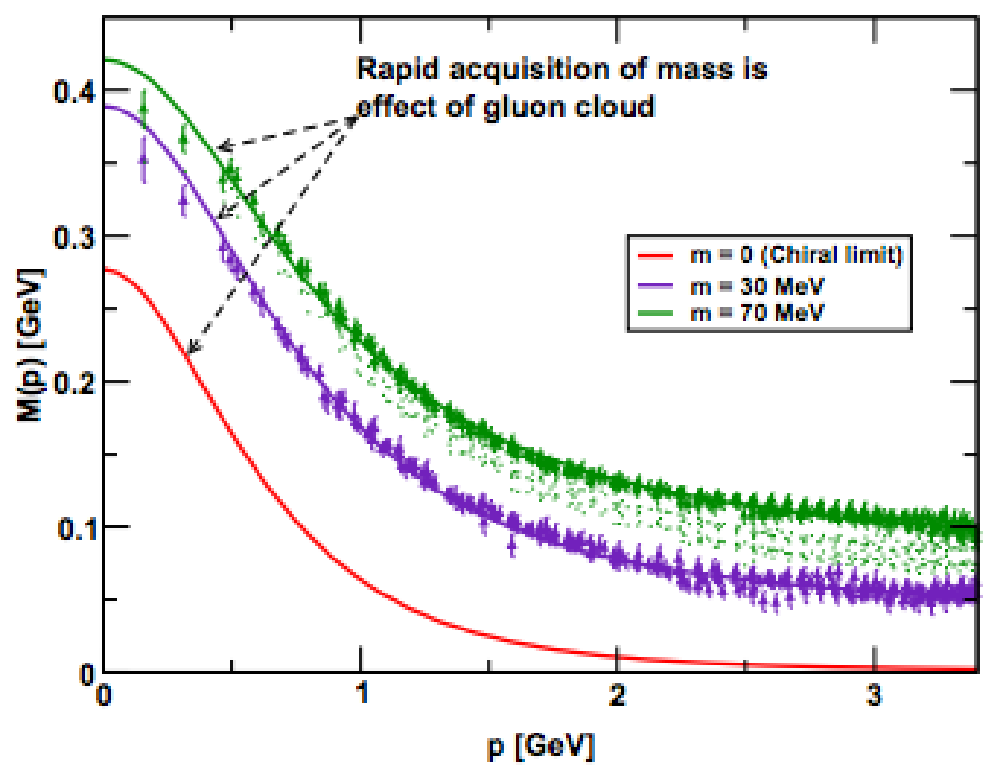

Figure 1.8: Dressed-quark mass function, $\mathrm{M}(\mathrm{p})$ : solid curves Dyson-Schwinger equations (DSE) results, obtained as explained in [3, 4]. Points are numerical simulations of lattice-QCD [5]. (NB. $\mathrm{m}=70 \mathrm{MeV}$ is the uppermost curve and current-quark mass decreases from top to bottom.) One observes the current-quark of perturbative QCD evolving into a constituent-quark as its momentum becomes smaller. The constituent quark mass arises from a cloud of low-momentum gluons attaching themselves to the current-quark. This is dynamical chiral symmetry breaking (DCSB): an essentially non perturbative effect that generates a quark mass from nothing; namely, it occurs even in the chiral limit. 

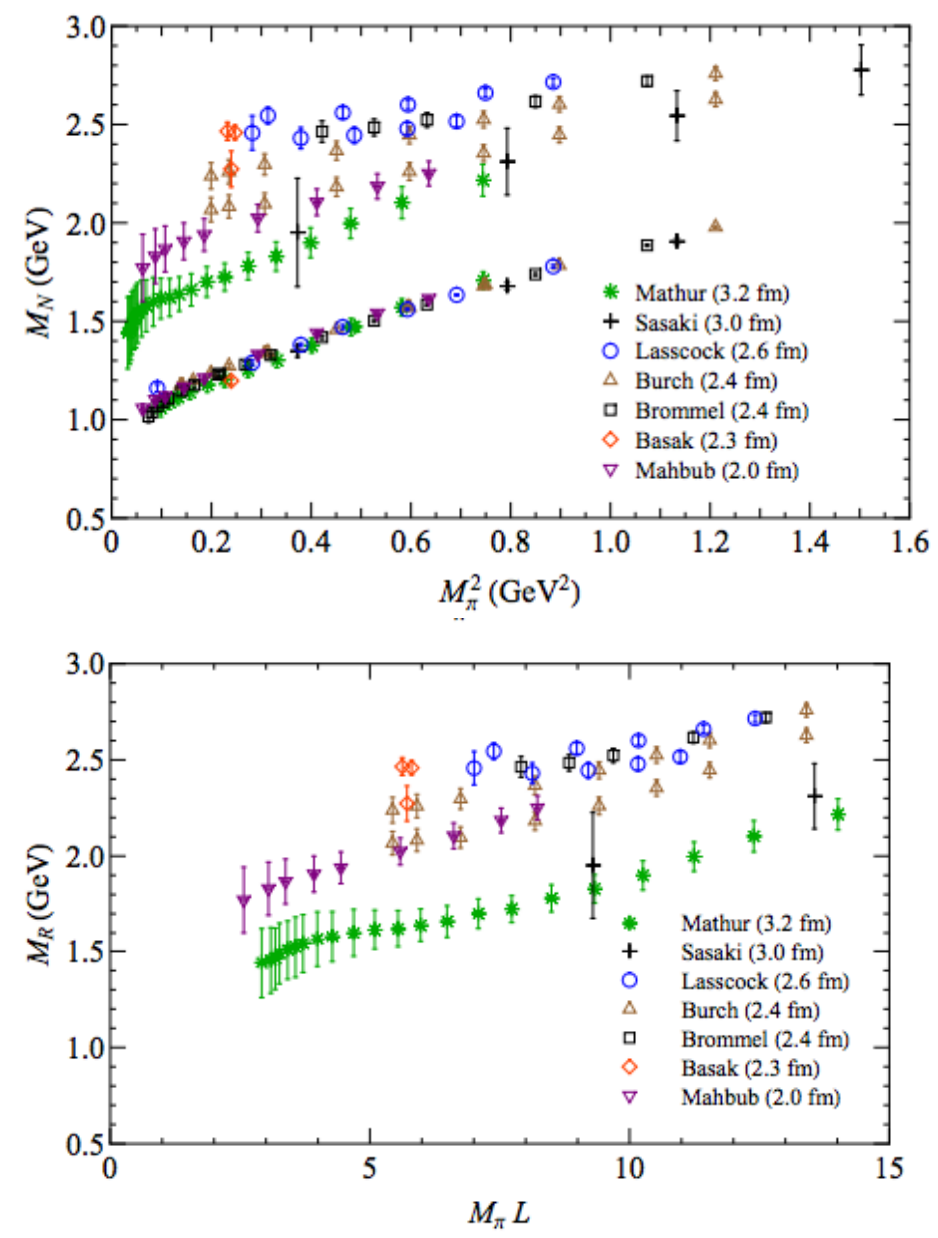

Figure 1.9: Summary of published LQCD calculations of the nucleon mass in GeV (top) as functions of $m_{\pi}^{2}$ and Roper mass as a function of the dimensionless product of the pion masses and lattice size (bottom). Note that the error bars are only statistical. [12]. 


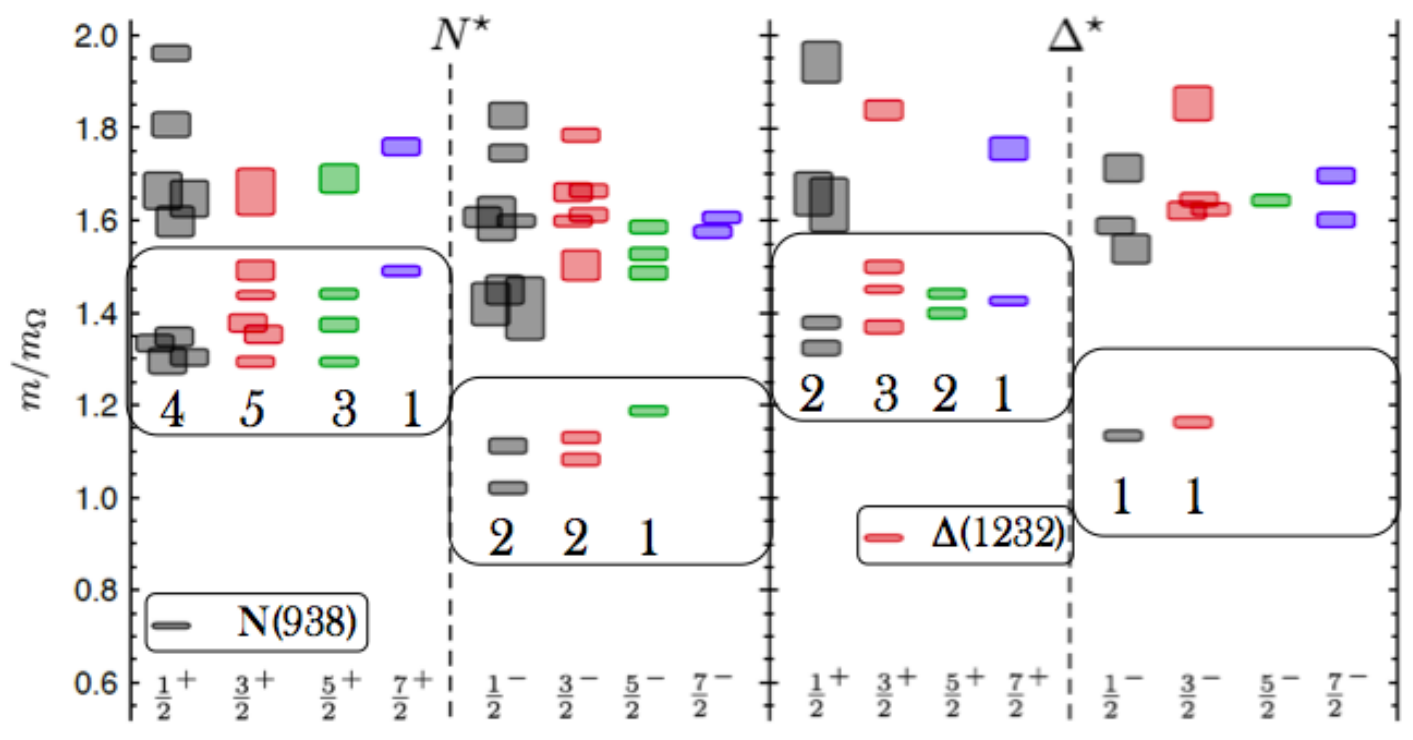

Figure 1.10: Spin-identified spectrum of Nucleons and Deltas from the lattices at $m_{\pi}=396 \mathrm{MeV}$, in units of the calculated $\Omega$ mass [11]. 
initial and final state, but they could be observed in other reactions where other initial e/o final states are investigated.

This observation is one of the major motivation behind the world-wide program for the study of photon- and electro-induced reactions. The price to pay with respect to meson-induced reaction is that the electromagnetic cross sections are much smaller than the hadronic ones and, even more important, photon-induced reactions can have significant non-resonant contribution identified as background. For example nucleon Born terms or vector meson exchange terms complicate the extraction of the resonance properties, therefore is mandatory to use reliable reaction models for the analysis of the photoproduction data.

Nowadays two different techniques are used to produce photon beams: Bremsstrahlung and Compton backscattering. In the former case, the electron beam from the accelerator hits a radiator; scattered electrons produce Bremsstrahlung with the typical $1 / E_{\gamma}$ spectral distribution. In the latter case, photons from a laser are scattered from electrons circulating in a storage ring. In both cases the energies of the photon and the scattered electron are correlated via the known incident electron and photon energy is currently used. The Bremsstrahlung technique is used at ELSA, JLAB (CLAS) and MAMI. Laser backscattering is employed at BNL (LEGS), at ESRF (GRAAL) and Sping8 (LEPS).

The advent of polarized beam and polarized target, has greatly enlarged the scope of photoproduction experiment, providing a direct access to the measurement of polarization observables, which permit to exploit at 360 degrees an intrinsic property of the matter: the spin.

\subsection{Polarization observables: exploiting the spin degree of freedom}

"Experiments with spin have killed more theories than any other single physical parameters" Elliot Leader.

An essential property for classifying particles is the "spin" or intrinsic angular momentum, whose existence was demonstrated in 1921 when the two physicists, named Otto Stern and Walther Gerlach, made an interesting experiment. They took a beam of electrically-neutral silver atoms and let it pass through a non-uniform magnetic field. The magnetic field deflected the silver atoms like it would do with little dipole magnets if you threw them through the field. After passing through the field, the deflected atoms hit a photografic plate and left a sign of their presence by creating little visible dots. The reason for using silver atoms is that they behave like hydrogen atoms with only one electron, but they are much easier to handle in the lab and to detect with a photo-plate. The result of this experiment was totally unexpected. The atoms which were coming out of an oven where silver was evaporated had no preferred orientation in space, therefore the spins of the outer 
electrons in these atoms should have pointed into all possible directions in space. Depending on their orientation, the magnetic force, that this little dipole magnets felt, was different and therefore the deflection for each of them was different too.

As a result, some of them would be oriented in a way that the deflection was very strong, others would have an orientation which results in almost no deflection at all. So Stern and Gerlach expected to find on the photographic plate a pattern like the one indicated in Fig. 1.11 as "Classical Expectation": a spot produced by several tiny dots caused by silver atoms hitting the plate all over the place.

They got instead something different: a pattern where only a contour was hit by the atoms and nothing in the middle. The explanation for that unexpected result was that the magnetic moments, and therefore the spins, can have only two certain orientations in space, that is they are quantized.

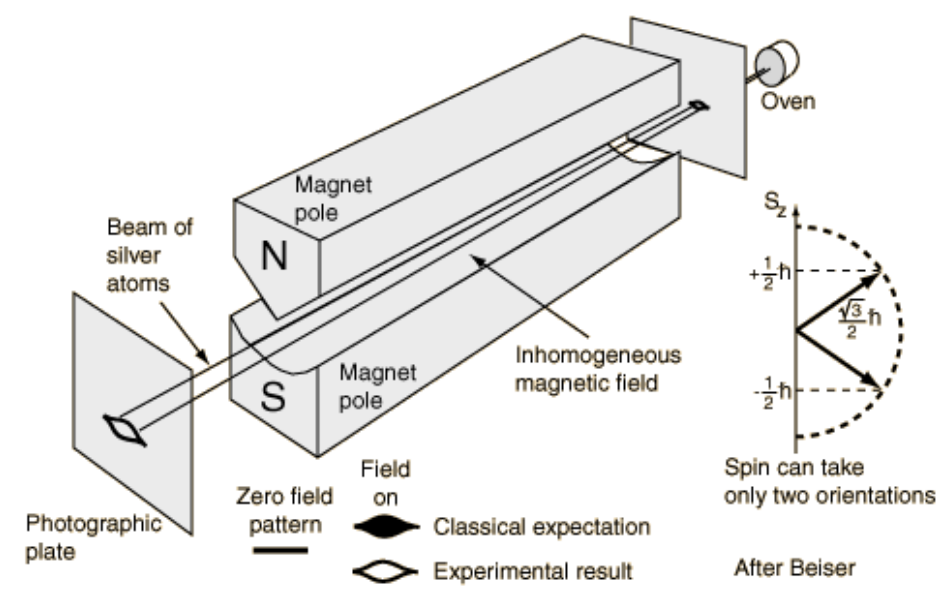

Figure 1.11: Stern-Gerlach experiment overview.

It is easy at this point imagining the analogy with a top spinning about an axis, just to recall to our mind something that we understand, but this is not what spin is. Spins are much more complex creatures. An intuitive but more effective way to look at the spin is to consider that it tells us something about the symmetry a particle has. A nice example is provided in Figure 1.12 [15].

A particle with spin 0 behaves like a dot as it looks the same way regardless of the direction from which you look at it. Something which has a symmetry like the playing card on the left needs a full rotation of $360^{\circ}$ until it looks again the same. That is the kind of symmetry a spin-1 particle has: after a full rotation it is again in the same state. A spin-2 particle behaves under rotation like the playing card on the right hand side. It already looks the same (that is, is in the same state) after 


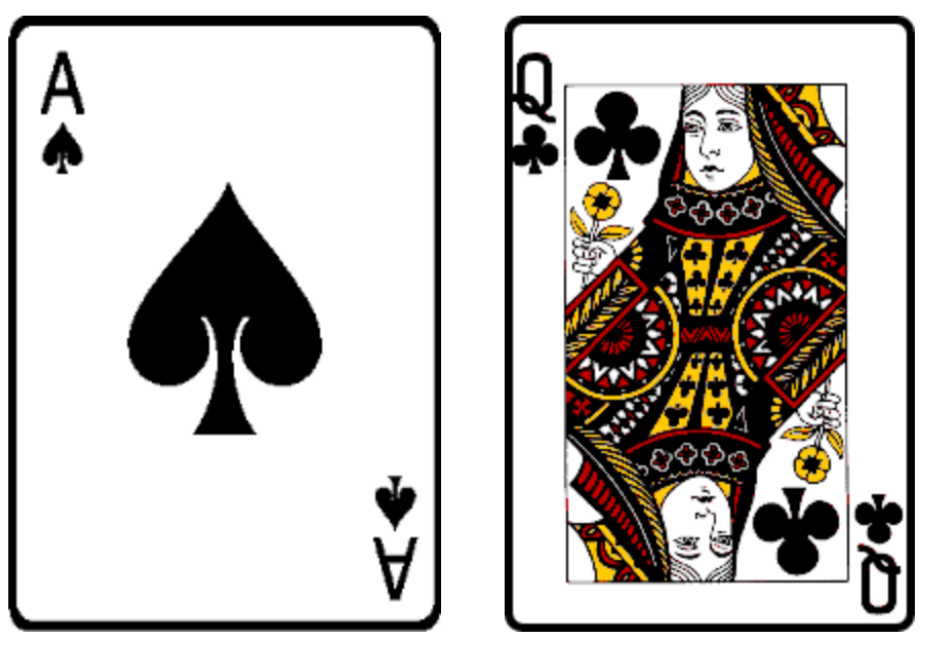

Figure 1.12: Spin 1 particles has, under rotation, the same symmetry property of the card on the left which needs a full rotation of $360^{\circ}$ degrees until it look again the same; spin 2 particles are like the playing card on the right side: it looks the same after a rotation of $180^{\circ}$ [15].

half a rotation $\left(180^{\circ}\right)$. Similarly, particles with higher spin look the same turning them through smaller faction of a complete revolution. For the electron, which is a spin $1 / 2$ particle, two full rotations are instead needed to bring it back to the same state, $\left(2 \times 360^{\circ}=720^{\circ}\right)$.

Ultimately the spin of a particle is an "internal" degree of freedom, it is intrinsically associated with the particle and determines the statistical behavior of the particle itself: particles possessing half-integer spin will obey the Fermi-Dirac statistic and they are named "Fermions". Those are what the world is made of: fundamental particle of spin $1 / 2$.

Particles possessing integer spin will obey Bose-Einstein statistic and will be called "Bosons": all the forces we know are mediated by these particles.

But other than a way to classify particles, spin can do much more for us. This property plays a crucial role in the dynamics of a reaction; the question that naturally arises is: how do can we exploit the spin information?

If we consider the photoproduction reaction of two pions from the nucleon:

$$
\gamma N \rightarrow \pi_{1} \pi_{2} N ;
$$

one important quantity associated to the reaction is the helicity of a particle, 
which is defined as the projection of the spin along the particle's momentum direction. The helicities involved in reaction 1.2 can have the values $\lambda_{\gamma}= \pm 1$ for the real photon, $\lambda_{N}= \pm 1 / 2$ and $\lambda_{N^{\prime}}= \pm 1 / 2$ for the initial and final state nucleon, respectively. Therefore eight matrix elements are possible:

$$
H_{\lambda_{N^{\prime}}, \lambda_{N}-\lambda_{\gamma}}=<\lambda_{N^{\prime}}|T| \lambda_{\gamma} \lambda_{N}>
$$

where $\mathrm{T}$ is the transition amplitude.

In case of the photoproduction of a single pion these eight helicity amplitudes are reduced, by parity invariance, to four helicity amplitudes:

$$
\begin{aligned}
& H_{1}=H_{+1 / 2,+3 / 2}=+H_{-1 / 2,-3 / 2} \\
& H_{2}=H_{+1 / 2,+1 / 2}=-H_{-1 / 2,-1 / 2} \\
& H_{3}=H_{-1 / 2,+3 / 2}=-H_{+1 / 2,-3 / 2} \\
& H_{4}=H_{+1 / 2,-1 / 2}=+H_{-1 / 2,+1 / 2}
\end{aligned}
$$

Taking combinations of these four helicity amplitudes gives sixteen possible quantities, all real and measurable. The sixteen observables are not all independent, as many relations between them hold, known as "Fierz Identities"; these relations reduce the number of observables which is necessary to measure to eight. Some of them are:

$$
\begin{aligned}
& d \sigma / d \Omega \sim\left|H_{1}\right|^{2}+\left|H_{2}\right|^{2}+\left|H_{3}\right|^{2}+\left|H_{4}\right|^{2} \\
& \Sigma \sim \operatorname{Re}\left(H_{1} H_{4}^{*}-H_{2} H_{3}^{*}\right) \\
& T \sim \operatorname{Re}\left(H_{1} H_{2}^{*}+H_{3} H_{4}^{*}\right) \\
& P \sim \operatorname{Re}\left(H_{1} H_{3}^{*}-H_{2} H_{4}^{*}\right)
\end{aligned}
$$

Where $d \sigma / d \Omega$ is the unpolarized differential cross section, $\Sigma$ is the photo beam asymmetry which arises if the photon beam is linearly polarized, $\mathrm{T}$ is the target asymmetry which arises if the target is longitudinally polarized and finally $\mathrm{P}$ is the recoil nucleon polarization. The unpolarized differential cross section is calculated by averaging the spin states of the particles in the initial state and summing over those of the final state. In this averaging process details of the interaction are often lost. Moreover, by looking at the first equation in 1.5 one can see that if, for example, the amplitude $H_{1}$ is small its (possibly interesting) contribution will be covered by other bigger amplitude contributions in the differential cross section. Conversely, if $H_{4}$ is large, the structure of $H_{1}$ would be emphasized in the photon beam asymmetry $\Sigma$ through the interference with $H_{4}$. We can state that polarization observables effectively measure interference and are therefore sensitive to small effects. Ultimately 
they provide a way to recover precious informations, in contrast to the measurement of a cross section alone which provides only limited information about a reaction. Sometimes transversely amplitudes can also be used instead of helicity amplitudes; they are expressed as a combination of the helicity amplitudes themselves. The only difference is that in this case the axis of quantization is chosen perpendicular to the scattering plane. A certain advantage of this representation is that the differential cross section and the single polarization observables can be expresses as linear combinations of the sums of the squared amplitudes rather than as bilinear functions of the amplitudes.

Since, as we saw above, for the photoproduction of a single pion there are eight independent observables, it turns out that complete information about the reaction mechanism, the so-called "complete experiment", requires the determination of seven independent real quantities (since the overall phase is arbitrary) at each kinematic point choosing, for example, the value of the incident photon energy and the mesons emissions angles. For discussion about the kind of measurements needed the interested reader is remanded to the work of [22], [23].

For the photoproduction reaction of two pseudoscalar pions, the invocation of parity invariance doesn't allow to reduce the number of helicity amplitudes ${ }^{2}$. Taking all combinations of the eight helicity amplitudes gives sixty-four polarization observables; by using 28 relations among these observables arising from consideration of absolute magnitudes of the helicity or transversity amplitudes and another 21 from consideration of their phases, it leaves 15 independent quantities. It turns out that a complete experiment in case of photoptoduction of two pions requires the measurement of at least 15 observable of single, double an triple polarizations, using both linearly and circularly polarized photon beams, in addition to the differential cross section.

The polarization observables which arise for the single- and two- pion photoproduction processes are given in tables 1.13 and 1.14 both from [21], for an unpolarized photon beam and for a circularly-polarized photon beam; polarization observables which require measurements linearly-polarized photons are not reported, since they are not relevant to this theses work. The interested reader is remanded to [21] for a complete list of all the possible polarization observables. The polarization observables are reported as a function of both helicity (indicated with a calligraphic M) and transversity amplitudes (indicated with b), defined as above. In the last columns the type of the experiment needed to measure the corresponding observable is indicated; for example $\mathrm{T}$ means that a polarized target is required, $\mathrm{R}$ requires an unpolarized target but the recoil polarization must be measured and so on.

These observables can be related to the reaction rate for the two processes of single- and double-pion photoproduction. In order to do that, it is convenient to

\footnotetext{
${ }^{2}$ The parity relations in case of the photoproduction of two pseudoscalar mesons can be only used to determine which observables are even or odd under the transformation $\Phi \rightarrow 2 \pi-\Phi[21]$.
} 


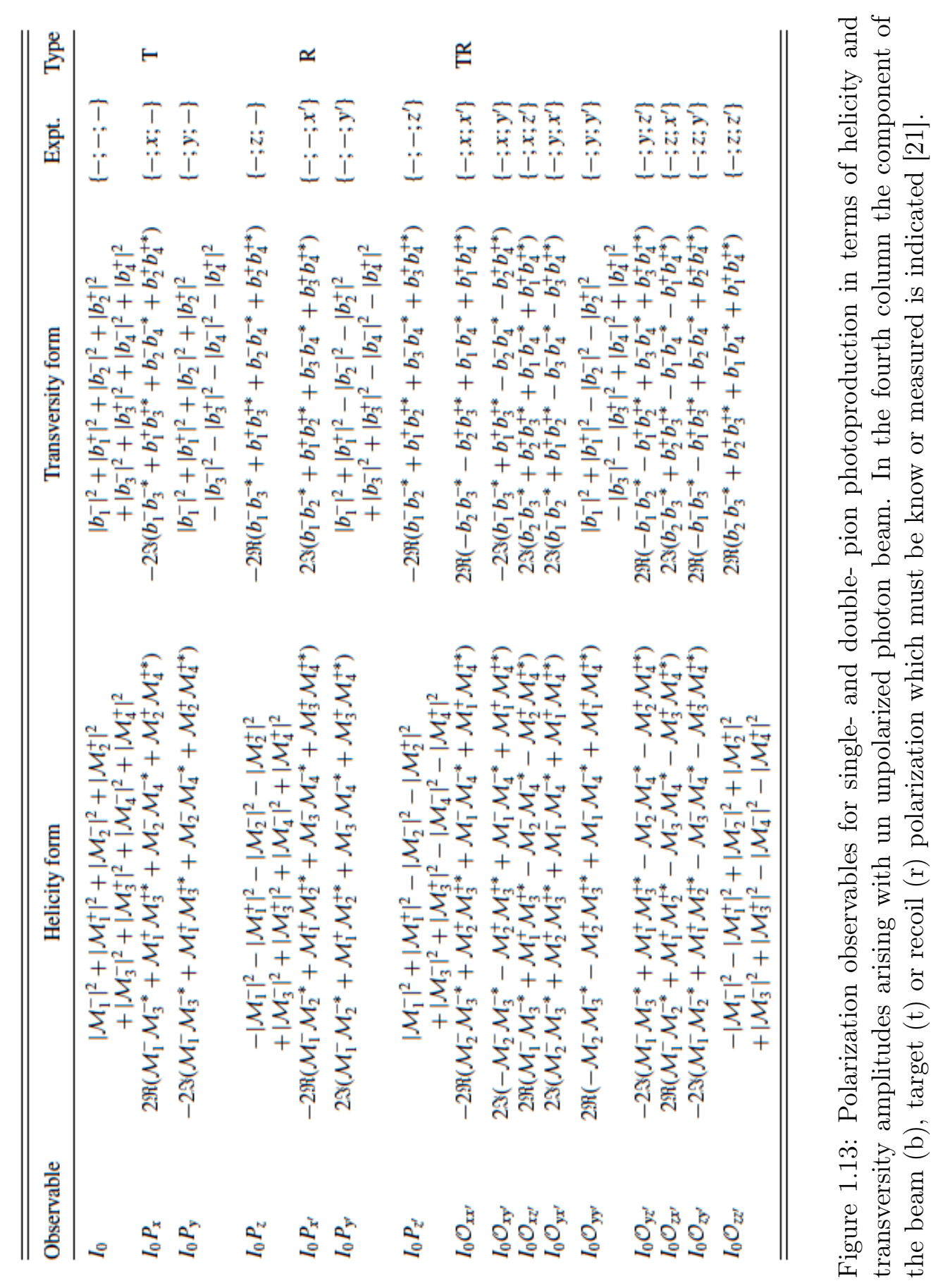




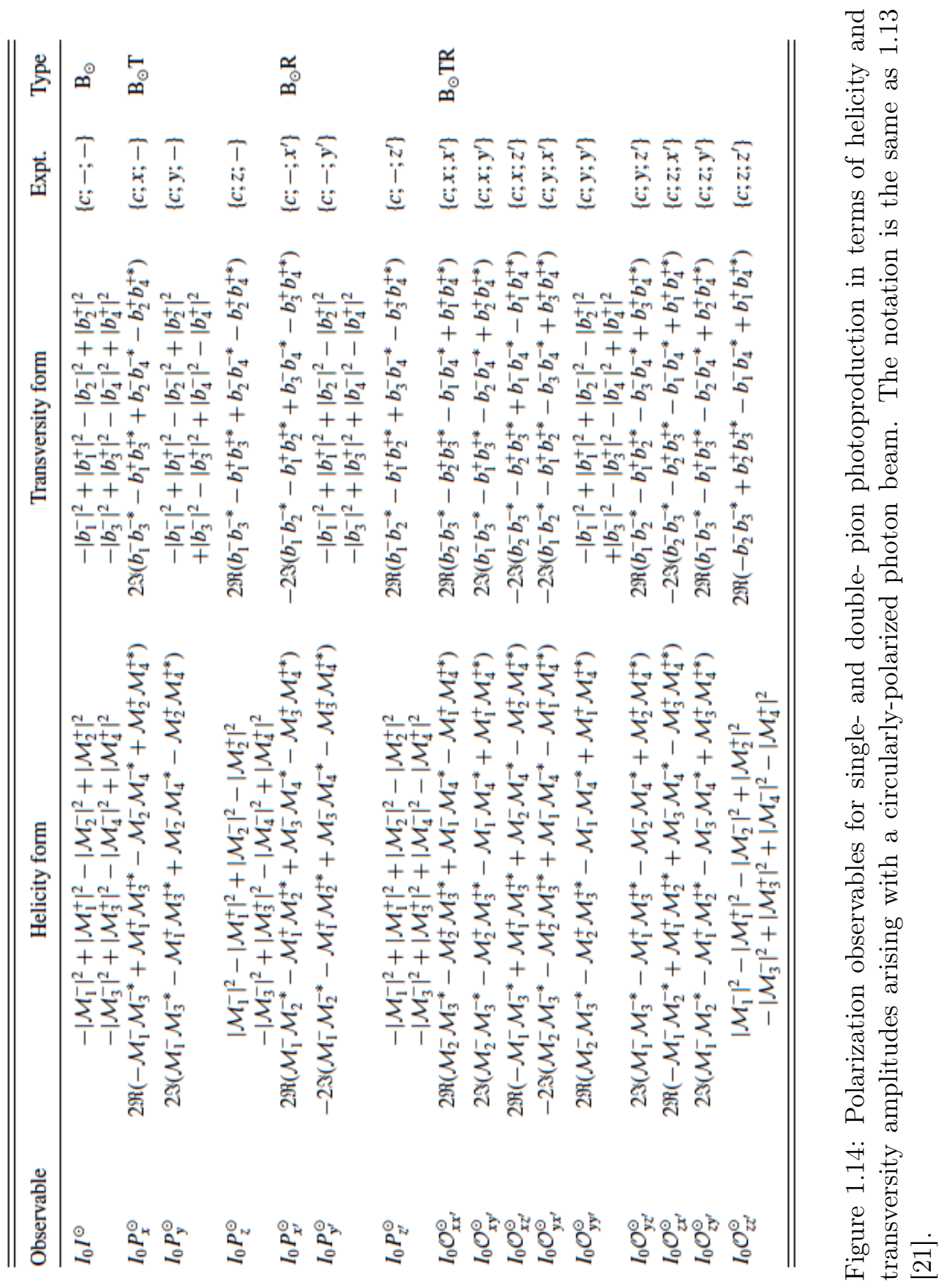


introduce the following kinematic variables in the center-of-mass frame:

- $\mathbf{K}$ is the tri-momentum of the photon beam particle.

- $\mathbf{Q}$ is the tri-momentum of the pion, in case of a single pion photoproduction, or the pair of pions, in case of a double pion photoproduction.

- $\mathbf{P}=-\mathbf{Q}$ is the tri-momentum of the recoil nucleon.

We can define the following set of axes:

$$
\hat{z}=\hat{\mathbf{K}} \quad \hat{y}=\frac{\mathbf{K} \times \mathbf{Q}}{|\mathbf{K} \times \mathbf{Q}|} \quad \hat{x}=\hat{y} \times \hat{z}
$$

It is also useful to define a set of axes in which the $z^{\prime}$ axis is parallel to the momentum of the recoil nucleon. In this system, the y axis coincides with the y axis of the collision plane. In terms of momenta, the axes for this system are:

$$
\hat{z}^{\prime}=\hat{\mathbf{P}} \quad \hat{y}^{\prime}=\frac{\mathbf{P} \times \mathbf{K}}{|\mathbf{P} \times \mathbf{K}|} \quad \hat{x}^{\prime}=\hat{y}^{\prime} \times \hat{z}^{\prime}
$$

The polarization observables are related to the reaction rate I as follows [21]:

$$
\begin{array}{r}
\rho_{f} I=I_{0}\left\{\left(1+\vec{\Lambda}_{i} \cdot \vec{P}+\vec{\sigma} \cdot \vec{P}^{\prime}+\Lambda_{i}^{\alpha} \sigma^{\beta^{\prime}} O_{\alpha \beta^{\prime}}\right)\right. \\
+\delta_{\odot}\left(I^{\odot}+\vec{\Lambda}_{i} \cdot \vec{P} \odot+\vec{\sigma} \cdot P^{\odot^{\prime}}+\Lambda_{i}^{\alpha} \sigma^{\beta^{\prime}} O_{\alpha \beta^{\prime}}^{\odot}\right) \\
+\delta_{l}\left[\sin 2 \beta\left(I^{s}+\vec{\Lambda}_{i} \cdot \vec{P}^{s}+\vec{\sigma} \cdot \vec{P}^{s^{\prime}}+\Lambda_{i}^{\alpha} \sigma^{\beta^{\prime}} O_{\alpha \beta^{\prime}}^{\odot}\right)\right. \\
\left.\left.+\cos 2 \beta\left(I^{c}+\vec{\Lambda}_{i} \cdot \vec{P}^{c}+\vec{P}^{c^{\prime}}+\Lambda_{i}^{\alpha} \sigma^{\beta^{\prime}} O_{\alpha \beta^{\prime}}^{c}\right)\right]\right\}
\end{array}
$$

where:

- $\vec{\Lambda}_{i}$ denotes the polarization of the initial nucleon

- $\vec{P}$ represents the polarization asymmetry that arises if the target nucleon is polarized

- $\rho_{f}=\frac{1}{2}\left(1+\vec{\sigma} \cdot \vec{P}^{\prime}\right)$ is the density matrix of the recoiling nucleon

- $O_{\alpha \beta^{\prime}}$ is the observable if both the target and the recoil polarization are measured; the prime indicates that the recoil observable is measured with respect to a set of axis $x^{\prime}, y^{\prime}, z^{\prime}$ where $z^{\prime}$ is along the direction of motion of the recoiling nucleon and $y^{\prime}=y$

- $\delta_{\odot}$ is the degree of circular polarization of the photon beam 
- $\delta_{l}$ is the degree of linear polarization, with the direction of polarization being at an angle $\beta$ to the $\mathrm{x}$-axis

- $I^{\odot}$ describes the beam asymmetry for an unpolarized target and a circularlypolarized photon beam

- $I^{c}$ describes the photon beam asymmetry for an unpolarized target and a linearly-polarized photon beam

- $I^{s}$ describes the photon beam asymmetry for an unpolarized target and a linearly-polarized photon beam

- $P^{\odot}$ and $P^{s, c}$ describes the polarization observables if, in addition to the target nucleon, the incoming photon is also polarized, either circularly or linearly, respectively

As for the photoproduction reaction of two pseudoscalar mesons we have 64 possible polarization observables, a minimum of eight measurement must be made at each kinematic point to obtain the absolute magnitudes of the helicity or transversity basis amplitudes. The eight phases of the transversity amplitude mean that there are seven independent phase differences that can be extracted, and seven measurements are needed. A complete set of experiments will therefore require measurement of single, double and triple polarization observables, in addition to the differential cross section.

The case of vector meson photoproduction is much more complicated: the spin of the meson contributes three additional degrees-of-freedom, so we have 2 spin states from the photon $\left(\lambda_{\text {gamma }}= \pm 1\right), 2$ spin states from the initial nucleon $\left(\lambda_{n}= \pm 1 / 2\right), 2$ spin states from the final nucleon $\left(\lambda_{n^{\prime}}= \pm 1 / 2\right)$ and three spin states from the vector meson $\left(\lambda_{V}= \pm 1,0\right)$; this leads to 24 complex numbers. However, 12 relations due to parity invariance hold between these numbers, leaving 12 independent complex helicity numbers or 24 numbers at each energy and angle. Similarly to the pseudoscalar case, we denotes these helicity amplitudes as:

$$
H_{a \lambda_{V}}(\theta)=\left\langle\lambda_{V} \lambda_{2}|T| \lambda \lambda_{1}\right\rangle
$$

where $\mathrm{a}=1, \ldots, 4$.

The vector meson amplitude can then be written as a $6 \times 4$ matrix in helicity space:

$$
\mathfrak{F}=\left(\begin{array}{cccc}
H_{21} & H_{11} & H_{3^{\prime}-1} & -H_{4-1} \\
H_{41} & H_{31} & -H_{1-1} & H_{2^{\prime}-1} \\
H_{20} & H_{10} & -H_{30} & H_{2-1} \\
H_{40} & H_{30} & H_{10} & -H_{20} \\
H_{2-1} & H_{1-1} & H_{31} & -H_{41} \\
H_{4-1} & H_{3-1} & -H_{11} & H_{21}
\end{array}\right)
$$


where we used the Jacob Wick parity relations.

The rows and the columns for 1.10 are labeled for the final state helicities $\left(\lambda_{V}, \lambda_{2}\right)$ and by the initial photon and nucleon helicities $\left(\lambda, \lambda_{1}\right)$. More specifically, the helicity amplitudes in 1.10 are defined as follows:

$$
\begin{aligned}
& H_{1 \lambda_{V}} \equiv\left\langle\lambda_{V,} \lambda_{2}=+1 / 2|T| \lambda=1, \lambda_{1}=-1 / 2\right\rangle \\
& H_{2 \lambda_{V}} \equiv\left\langle\lambda_{V,} \lambda_{2}=+1 / 2|T| \lambda=1, \lambda_{1}=+1 / 2\right\rangle \\
& H_{3 \lambda_{V}} \equiv\left\langle\lambda_{V,} \lambda_{2}=-1 / 2|T| \lambda=1, \lambda_{1}=-1 / 2\right\rangle \\
& H_{4 \lambda_{V}} \equiv\left\langle\lambda_{V,} \lambda_{2}=-1 / 2|T| \lambda=1, \lambda_{1}=+1 / 2\right\rangle
\end{aligned}
$$

One should note that the simpler case of pseudoscalar mesons would be recovered by taking only a subset $\lambda_{V}=0(2 \times 4)$ of the above matrix. The number of observables increases to 8 single, 51 double, 123 triple and 108 quadruple polarization observables for a total of 290 observables. It turns out that a complete measurements in this case is absolutely out of reach.

This makes the vector meson photoproduction a hard subject to study, and this is possibly the reason why the scientific literature is so poor compared to that published on the pseudoscalar mesons.

The general formalism of polarization observables has been treated in details in the authoritative work of Pichowsky et al. [24].

\subsubsection{Another degree of freedom: the Isospin}

An extra complication appears if one consider another degree of freedom: the isospin. The amplitudes for the photoproduction of pseudoscalar mesons have three components, which arise from the iso-scalar and iso-vector nature of the photon field. For photoproduction reactions on the proton and neutron targets these amplitude can be written [28]:

$$
\begin{aligned}
& A_{\gamma p \rightarrow\left(\begin{array}{c}
\pi^{0} p \\
K^{+} \Sigma^{0}
\end{array}\right)}=\quad \mp\left[\frac{1}{\sqrt{3}} \mathbf{A}^{(\mathbf{0})} \underset{(\underset{K \Sigma}{\pi N})}{ }-\frac{1}{3} \mathbf{A}^{(\mathbf{1})}\left(\begin{array}{c}
\pi N \\
K \Sigma
\end{array}\right)\right]^{\left(I=\frac{1}{2}\right)}+\frac{2}{3} \mathbf{A}_{\left(\begin{array}{c}
I=3 / 2) \\
K \Sigma
\end{array}\right)}^{(N)} \\
& A_{\gamma p \rightarrow\left(\begin{array}{c}
\pi^{+} n \\
K^{0} \Sigma^{+}
\end{array}\right)}= \pm \sqrt{2}\left[\frac{1}{\sqrt{3}} \mathbf{A}^{(\mathbf{0})} \underset{\substack{\pi N \\
K \Sigma}}{\pi N}-\frac{1}{3} \mathbf{A}^{(\mathbf{1})}\left(\begin{array}{c}
\pi N \\
K \Sigma
\end{array}\right)\right]^{\left(I=\frac{1}{2}\right)}+\frac{\sqrt{2}}{3} \mathbf{A}_{\left(\begin{array}{c}
\pi N \\
K \Sigma
\end{array}\right)}^{(I=3 / 2)} \\
& A_{\gamma p \rightarrow\left(\begin{array}{c}
\eta p \\
K^{+} \Lambda
\end{array}\right)}=\quad+\left[\mathbf{A}^{(\mathbf{0})}\left(\begin{array}{c}
\eta N \\
K \Lambda
\end{array}\right)-\frac{1}{\sqrt{3}} \mathbf{A}^{(\mathbf{1})}\left(\begin{array}{c}
\eta N \\
K \Lambda
\end{array}\right)\right]^{\left(I=\frac{1}{2}\right)}
\end{aligned}
$$




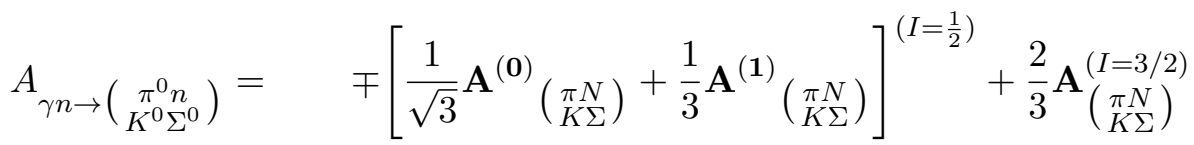

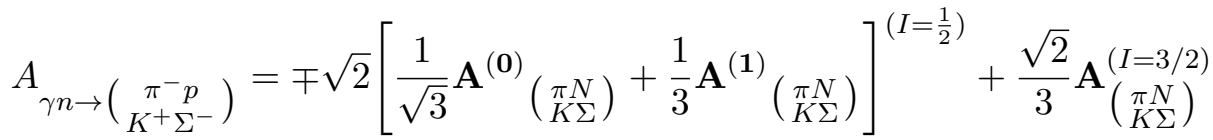

$$
\begin{aligned}
& A_{\gamma n \rightarrow\left(\begin{array}{c}
\eta n \\
K^{0} \Lambda
\end{array}\right)}=\quad+\left[\mathbf{A}^{(\mathbf{0})}\left(\begin{array}{c}
\eta N \\
K \Lambda
\end{array}\right)+\frac{1}{\sqrt{3}} \mathbf{A}^{(\mathbf{1})}\left(\begin{array}{c}
\eta N \\
K \Lambda
\end{array}\right)\right]^{\left(I=\frac{1}{2}\right)}
\end{aligned}
$$

Here the $\mathbf{A}^{(\mathbf{0})}$ and $\mathbf{A}^{(\mathbf{1})}$ components result from coupling the $\mathrm{I}=1 / 2$ nucleon with the iso-scalar and iso-vector components of the photon field to yield a total isospin of $I=1 / 2$. The terms in the square bracket are the projections of the isospin $1 / 2 \mathrm{am}-$ plitudes onto proton (1.12) or neutron (1.13) targets. As one can see from the first of the equations 1.12 , these combinations with $\mathrm{I}=1 / 2$ are the same for the two different charged channels of $\pi$ and K production from a proton target alone. It follows that measurements of the two charged channels in $\pi$ or $\mathrm{K}$ production from protons alone are sufficient to isolate the $\mathrm{I}=3 / 2$ amplitudes, which are associated with the $\Delta$ states, but they are not enough to disentangle the amplitude components $\mathbf{A}^{(\mathbf{0})}$ and $\mathbf{A}^{(\mathbf{1})}$. The same holds for the two $\mathrm{I}=1 / 2$ amplitudes which contribute to photoproduction of $\eta$ or $\Lambda$. If one looks at the amplitudes for the same photoproduction processes but on a neutron target (equation 1.13), one can see that the two $I=1 / 2$ amplitudes appear with opposite signs. This implies that it is necessary to perform measurements on both proton and neutron targets to separate the contributions of the amplitudes $\mathbf{A}^{(\mathbf{0})}$ and $\mathbf{A}^{(\mathbf{1})}$.

The total photoabsorption cross sections for the reaction $\gamma N \rightarrow N X$ on the proton and the neutron, show different structures (fig. 1.15 ) [29]. The latter was measured in a quasi-free kinematic from the deuteron target. In this figure the cross section was fitted with a smooth background and Breit-Wigner curves for the nucleon excited states which are labeled as $L_{2 I 2 J}(W)$, where $\mathrm{W}$ is the mass of the state, $\mathrm{L}=0,1,2, \ldots$ the angular momentum for the decay into the $N \pi$ channel and I,J are the isospin and spin of the resonance, respectively.

These plots also confirm the difficulty in the identification and investigation of individual states from cross section measurement alone; indeed only the lowest lying excited state of the nucleon, the $\Delta$ resonance $\left(P_{33}(1232)\right)$, corresponds to an isolated peak in the spectrum. At masses around $1500 \mathrm{MeV}$, several resonances ( $\left.P_{11}(1440), D_{13}(1520), S_{11}(1535)\right)$ contribute to the broad structure observed in the spectrum. This energy regime is called the second resonance region. A third resonance region at $\mathrm{W} \sim 1600-1700 \mathrm{MeV}$ is clearly seen on the proton, but is less pronounced on the neutron target.

This isospin dependence of electromagnetic interactions is also seen by looking 


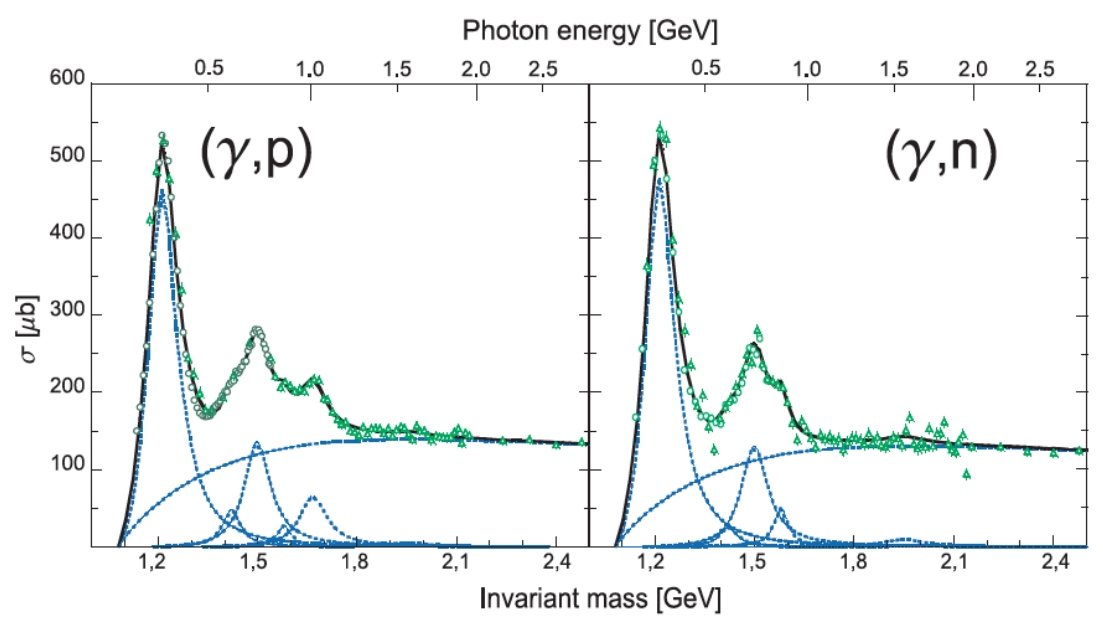

Figure 1.15: Cross section for total photo absorption on the proton (left hand side) and the neutrons (right end side) [29]. Points: measured data, curves: fit of Breit-Wigner shapes of nucleon resonance $\left(P_{33}(1232), P_{11}(1440)\right.$, $D_{13}(1520), S_{11}(1535), F_{15}(1680)$ (only for proton) and $F_{37}(1950)$ and a smoothly varying background [20].

at the difference of dependent cross sections, $\sigma^{1 / 2}-\sigma^{3 / 2}$. Predictions for these helicity difference cross sections in the $\gamma n \rightarrow \pi^{-} p$ channel from the SAID and MAID multipole analyses [25] are shown in upper part of Figure 1.16. The two partial wave analyses are similar below $800 \mathrm{MeV}$ but show different trends at higher energies. We observe that both the SAID solution [25], as well as the helicity-dependent total absorption data from Bonn [26], suggest a positive rise in the difference cross section from a neutron target at high energies, not seen in proton data [27], as shown in the the lower part of Figure 1.16. This could indicate the contributions from not yet identified resonances that may be weakly coupled to $\gamma \mathrm{p}$ but strongly coupled to $\gamma \mathrm{n}$ states.

Another -not less important- motivation to perform measurements both on proton and neutron targets is that, so far, only few meson production reactions off the neutron have been measured and, in general, the data have an inferior quality than the corresponding reactions off the free proton; this is due to the complications related to the neutron detection and on the kinematics obeyed by bond nucleons. 

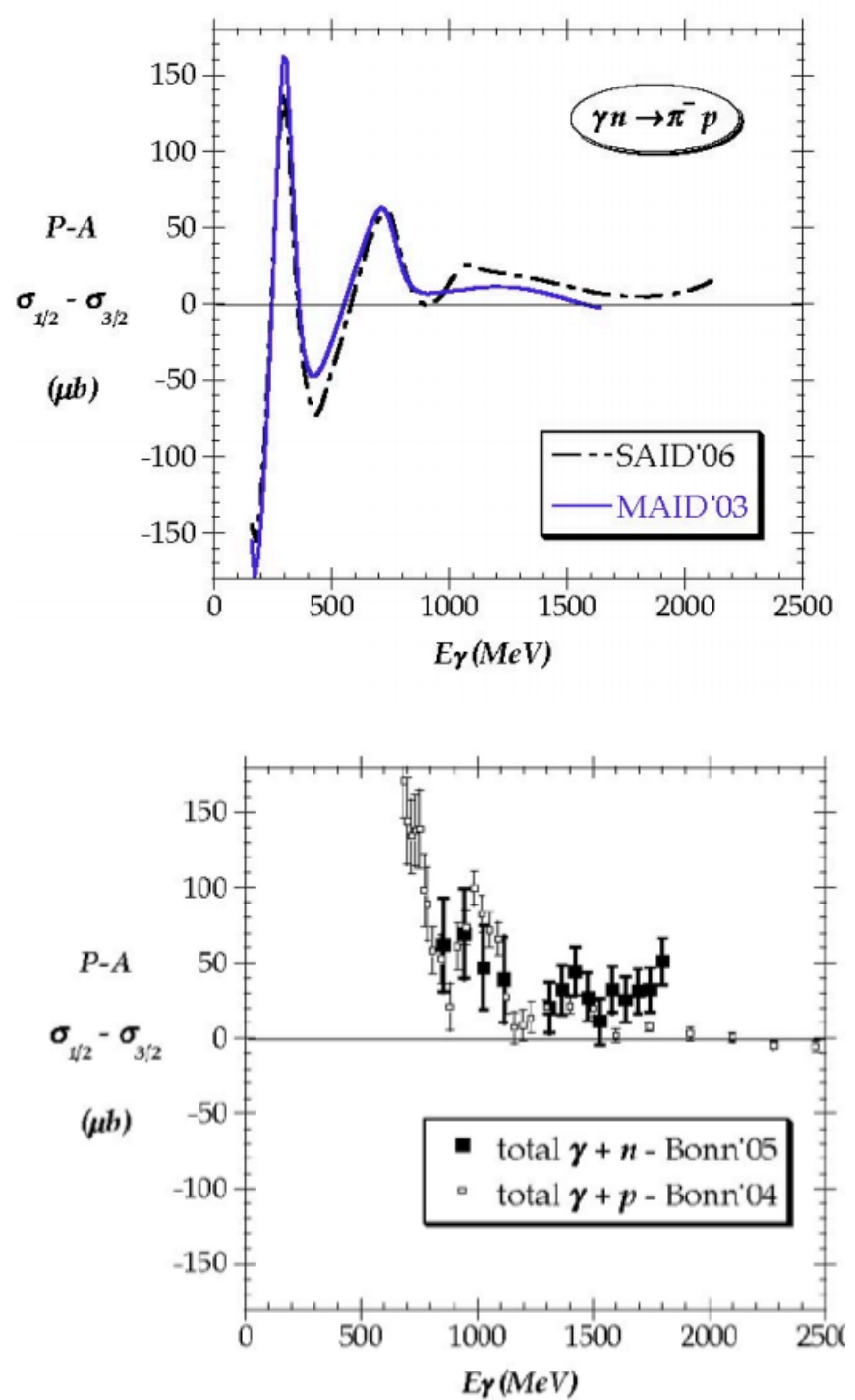

Figure 1.16: Left: helicity difference cross section for the reaction $\gamma n \rightarrow \pi^{-} p$ predicted by SAID and MAID PWA [25, 28]:. Right: helicity difference for $\mathrm{n}$ and $\mathrm{p}$ targets measured in Bonn [26, 28]. 


\subsection{Motivation for this analysis}

For this thesis work I used the data provided by the g14 experiment, which took place in the experimental Hall B of the Thomas Jefferson National Laboratory (JLab) between November 2011 and May 2012.

As it will be described with more details in chapter 2, the g14 experiment used the CLAS spectrometer, a beam of circularly or linearly polarized photons and a longitudinally polarized Hydrogen-Deuteride frozen spin target where both the proton and the neutron were polarized. The presence of polarized photons and polarized HD target give access to the measurement of several single and double polarization observables on both proton and neutron targets.

The present work is focused on the extraction of two of the polarization observables from the two-pion photoproduction reaction on the nucleon: the photon polarization asymmetry $I^{\odot}$, for both the reactions $\gamma p \rightarrow \pi^{+} \pi^{-} p$ and $\gamma n \rightarrow \pi^{+} \pi^{-} n$ and the helicity difference $P_{z}^{\odot}$, for the reaction $\gamma p \rightarrow \pi^{+} \pi^{-} p$. The results, where available, will be compared with data form two previous experiments, both performed at the JLab, such as g1c [71] and [72] and with the result of the model by A. Fix [73]. Finally, very preliminary results for the double polarization beam-target asymmetry E for the reaction $\gamma p \rightarrow \rho^{0} p$ will be shown.

This theses is organized as follows: chapters 2 and 3 will be dedicated to the description of the experimental setup used for the g14 experiment and of the HD-ice polarized frozen spin target, respectively. In chapter 4 the procedure followed for the selection of the final states $\pi^{+} \pi^{-} p$ and $\pi^{+} \pi^{-} n$ will be described. In the first part of chapter 5 the results for the extraction of the polarization observables $I^{\odot}$ and $P_{z}^{\odot}$ will be shown; in the second part the event selection for the reaction $\gamma p \rightarrow \rho^{0} p$ will be discussed and very preliminary results for the E asymmetry will presented. Chapter 6 will be dedicated to the conclusions. 


\section{Chapter 2}

\section{The experimental apparatus}

The data used for this work were taken from the g14 run period, which started on November 2011 and finished on May 2012 in Hall B at the Thomas Jefferson National Accelerator Facility, also known as Jefferson Laboratory (JLab), in Newport News, Virginia, USA. The g14 run period corresponds to the experiment E06-101 " $N^{*}$ Resonances in Pseudoscalar-meson photo-production from Polarized neutrons in $\vec{H} \vec{D}$ and a complete determination of the amplitude $\gamma n \rightarrow K^{0} \Lambda^{\prime \prime}[28]$. The experiment used a photon-tagging system, a Hydrogen-Deuteride (HD) polarized frozen-spin target and the CEBAF Large Acceptance Spectrometer (CLAS); it was the last of a long series of experiments performed during the so-called $6 \mathrm{GeV}$ era (being $6 \mathrm{GeV}$ the maximum energy for the electro-beam). In the tables 2.1 and 2.2 all the experiments performed during the $6 \mathrm{GeV}$ period are reported. In 2012 the upgrade of the facility to get a maximum electron energy of $12 \mathrm{GeV}$ started and a new detector, CLAS12, is under construction; a series of new experiments which will use this new experimental setup has been already proposed to start in 2016. In this chapter the various detector components used for the g14 running period will be described in details. 


\begin{tabular}{|l|l|}
\hline Run period & Title \\
\hline g1 & $\mathrm{N}^{*}$ and Mesons \\
\hline g2 & Deuterium Target \\
\hline g3 & Few Body and Nuclei \\
\hline g5 & Photoabsorption and Photofission in Nuclei \\
\hline g6 & $\mathrm{N}^{*}$ and Meson Properties \\
\hline g7 & Vector Mesons in Nuclei \\
\hline g8 & Vector Mesons with Polarized Photons \\
g9-Frost & Mesons with polarized target and photon beams \\
\hline g10 & Search for $\Theta^{+}$Pentaquark on Deuterium \\
\hline g11 & Search for $\Theta^{+}$Pentaquark and excited states on Hydrogen \\
\hline g12 & Hybrid Mesons and Exotic Baryon Search \\
\hline g13 & Kaon Production on the Deuteron using Polarized Photons \\
\hline g14 & Search for neutron resonances in hyperon production from polarized HD target \\
\hline
\end{tabular}

Table 2.1: Experiments performed at JLab using a photon beam.

\begin{tabular}{|l|l|}
\hline Run period & Title \\
\hline e1 & $\mathrm{N}^{*}$ and Mesons \\
\hline e1-dvcs & Deeply Virtual Compton Scattering on the proton \\
\hline e1-6 & $\mathrm{N}^{*}$ and Mesons at $6 \mathrm{GeV}$ \\
\hline e2 & Multi Nucleon \\
\hline e3 & $\mathrm{N}^{*}$ and Mesons with polarized targets \\
\hline e5 & $\mathrm{G}_{M}^{N}$ from electron-proton and electron-neutron scattering in Deuterium \\
\hline e6 & High Momentum Nucleons in Few Body System \\
\hline e8 & Barely off-shell Nucleon Structure \\
\hline eg1 & Polarized Target with electron beam \\
\hline eg1-dvcs & Deeply virtual processes with polarized targets \\
\hline eg2 & Quark propagation and Color Transparency in Nuclei \\
\hline eg3 & Search for cascade Pentaquarks \\
\hline eg4 & Gersaimov-Drell-Hearn sum \\
\hline eg5 & Two photon exchange processes \\
\hline eg6 & Hybrid meson search and DVCS in ${ }^{4} H e$ \\
\hline ET-1 & DVCS and SIDIS on transverse polarized target \\
\hline Primex & Pimakoff Experiment \\
\hline RadPhi & Radiative Phi Decay \\
\hline
\end{tabular}

Table 2.2: Experiments performed at JLab using an electron beam 


\subsection{CEBAF: Continuous Electron Beam Accelerator Fa- cility}

The Continuous Electron Beam Accelerator facility (CEBAF) is located at the Thomas Jefferson National Accelerator Facility, in Newport News, Virginia. There are currently three experimental areas, called Hall A, B, and C, respectively. The machine could deliver electrons to the three experimental areas at any multiple of one-fifth of the end point energy. The accelerator consisted of two antiparallel superconducting linear accelerator (LINACS) of $0.6 \mathrm{GeV}$ energy gain, connected at each end by nine recirculation arcs, capable to accelerate the electrons energies up to $6 \mathrm{GeV}$. The schematic view of the CEBAF is shown in Figure 2.1.

The electron beam was produced in an electron gun through photo-emission of electrons from a GaAs photocathode. The photo-emission process took place by illuminating the cathode with three RF gain-switched lasers independently pulsed at $499 \mathrm{MHz}$ and $120^{\circ}$ out of phase. This produced three independent electron beams, one for each experimental Hall. The three beams were then combined through a rotating-disk with three slits into a $1497 \mathrm{MHz}$ pulse train of electrons. The combined beam was then accelerated to $67 \mathrm{MeV}$ by two cryomodules and injected into the accelerator; passing through a linac the electron beam was accelerated up to 0.6 $\mathrm{GeV}$, gaining a total of $1.2 \mathrm{GeV}$ after one complete loop. Since the two linacs are connected to each other by nine recirculation arcs, the electron beam could pass five times through the linacs gaining a maximum energy of $6 \mathrm{GeV}$. The beam for each experimental Hall is then extracted through five sub-harmonic separator cavities operating at $499 \mathrm{MHz}$. The phasing of the separator cavities allowed to extract the beam in the following way: the four separator placed at the lower energy recirculating arcs where phased in such a way that only a portion of the beam was allowed to be steered to one of the experimental areas, while the remaining is recirculated back to the linacs. The phasing of the fifth separator on the high energy leg allowed to split the beam into three direction to be delivered to the three halls for simultaneous data taking. The electron beam had a $2.004 \mathrm{~ns}$ bunch structure with an energy spread of $\Delta E / E \leq 10^{-4}$. Hall $\mathrm{A}$ and $\mathrm{C}$ were equipped with two-arm spectrometers which allowed high-resolution measurements but a limited acceptance while Hall B was instrumented with a large-acceptance detector for multi-particle detection but had lower resolution. Since the g14 experiment took place in the Hall B, the rest of the chapter is entirely dedicated to the Hall B experimental setup. 


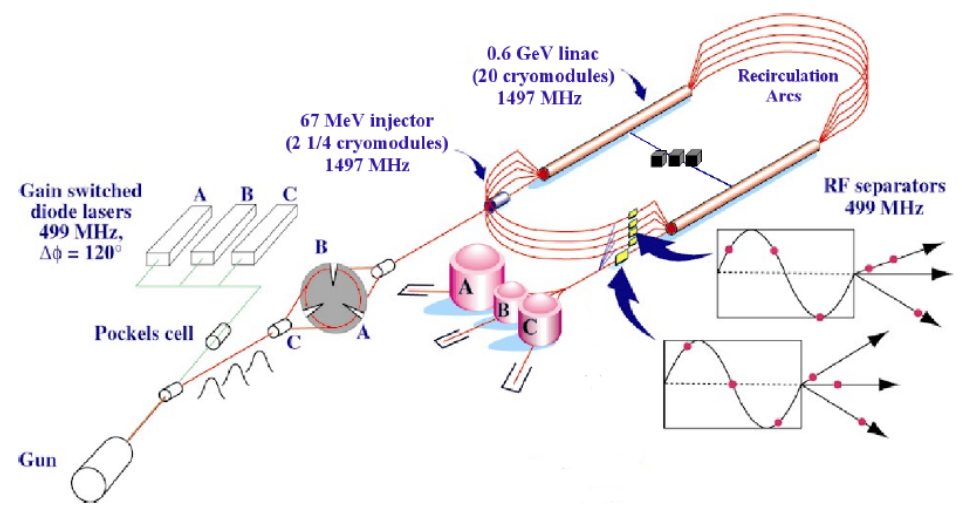

Figure 2.1: The Continuous Electron Beam Accelerator Facility at JLab.

\subsection{Experimental Hall B}

Hall B, where the g14 run has been performed, is the smallest of the four experimental areas at JLab. It housed the CEBAF Large Acceptance Spectrometer, known as CLAS, used for the g14 experiment. A schematic of Hall B is given in Figure 2.2. The experimental hall contained also the photon tagging spectrometer, the beamline, two pair spectrometers, two beam position monitors and the devices used for photon normalization.

In the following the various detector systems will be described in details. 


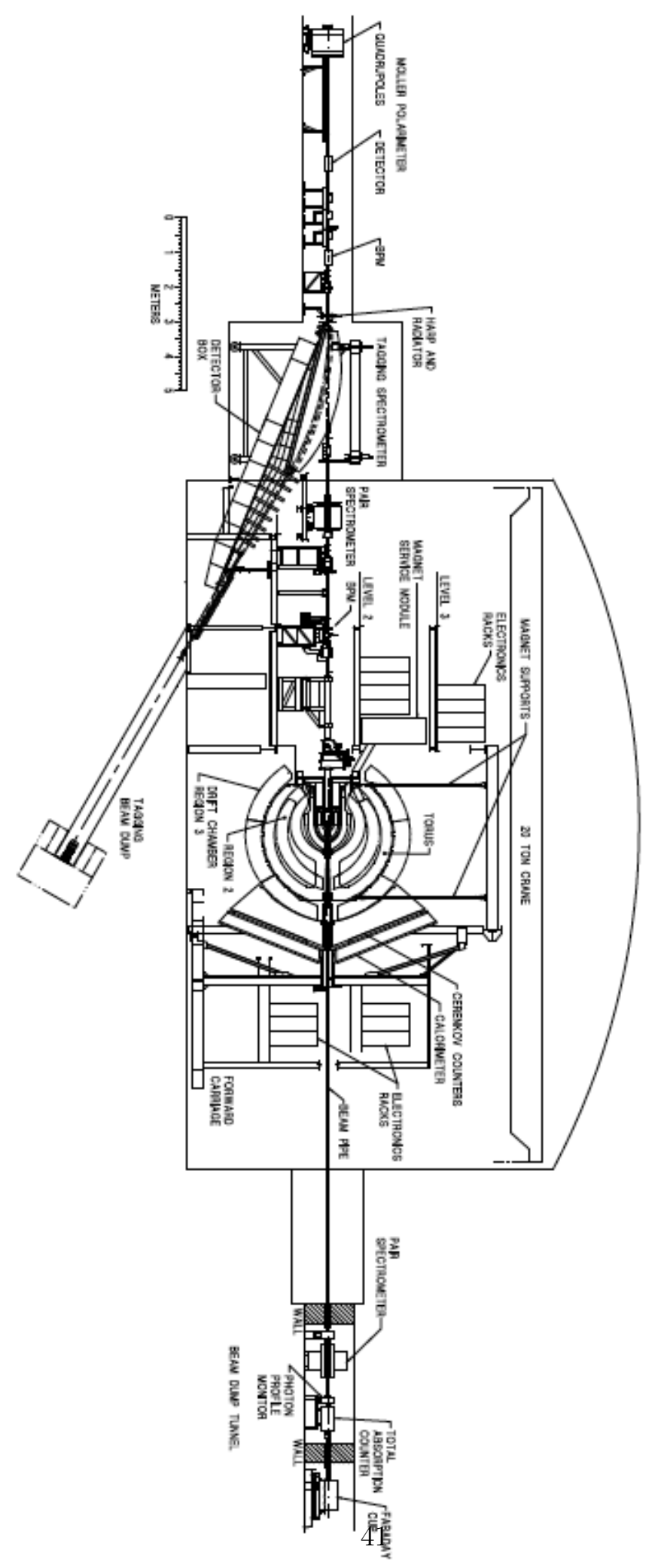

Figure 2.2: Schematic view of Hall B [30]. 


\subsection{Beamline Devices}

Several detectors placed along the CLAS beamline where used to monitor the electron and photon beam during the HDice experiment. These devices were used to measure the transverse position, the intensity of the electron beam and the intensity of the photon beam. The electron beam current, which was dumped after passing through the tagger and the detector, was measured by a Faraday cup, built with a 4 Ton block of lead placed on ceramic supports inside vacuum, at the end of the beamline.

\subsubsection{Beam Position Monitors}

Beam Position Monitors (BPM) were used to monitor the beam position during the data taking. The BMP operated using currents induced by the electron beam on wires, that was proportional to the distance of the beam from the wires; it allowed to measure the $\mathrm{x}$ and $\mathrm{y}$ position of the beam. The beam position was adjusted through a feedback loop during data-taking.

\subsubsection{Beam Profile Monitors}

The electron beam profile had to be determined whenever any changes were made to the beam, such as in the beam current for example. The monitoring of the beam profile was done during dedicated runs, since it's a destructive technique. At this scope, Hall B was equipped with three profilers, called harp scanners, positioned along the beamline. Each harp consisted of a set of thin wires $(20$ and $50 \mu \mathrm{m}$ tungsten and $100 \mu \mathrm{m}$ iron) orientated along the two directions perpendicular to the beam. During monitoring measurement, the harps were slid into the beamline and moved at $45^{\circ}$ through the beam by using a computer-controlled stepping motor to allow to take measurement at regular intervals across the beam. A small fraction of the electrons was scattered by the wire and the signal from the resulting Cerenkov light produced in the glass windows of the photomultiplier tubes (PMTs), which were positioned forwards from the harps, was used to map out the beam profile.

\subsubsection{Møller polarimeter}

For measuring the degree of polarization of circularly polarized photons it is necessary to measure the degree of longitudinal polarization of the electron beam. These measurement were carried out using the Møller polarimeter [38], which was moved into the beamline during dedicated measurements. The Møller polarimeter was a magnetic spectrometer consisting of a $25 \mu \mathrm{m}$ thick Permendur foil target magnetized to produce a $8 \%$ polarization of surface electrons along the plane of the foil, two quadrupole magnets downstream of the foil and two scintillator detectors coupled to PMTs. The value of the beam polarization could be obtained by measuring, with different target polarization orientations, the asymmetry of the scattered electrons 
resulting from elastic $M \oslash l l e r$ electron-electron scattering, which takes place in the foil. The magnets separated the scattered MØller electrons from the unscattered beam and directed them to a pair of lead/scintillator fibre composite detectors, positioned at both sides of the beam line. The longitudinal electron beam polarization is calculated as:

$$
P_{e}=\frac{A}{A_{z}} P_{T}
$$

where $\mathrm{A}$ is the asymmetry as measured by the Møller polarimeter, $A_{z}$ is the analyzing power of the target and $P_{T}$ is the target foil polarization.

\subsubsection{Pair Spectrometer}

The Pair Spectrometer was located $10 \mathrm{~m}$ downstream of the photon beam radiator, just upstream of CLAS; it consisted of a dipole magnet and two planes of scintillator counters, positioned symmetrically to the left and right of the beam axis, in the horizontal plane and downstream of the magnet. This device was used to measure the relative tagged photon flux over a wide range of intensities and operated continuously during the g14 experiment. During the linear running period it was useful for monitoring the flux stability of the tightly collimated photon flux.

\subsubsection{Total Absorption Shower Counter}

The total absorption shower counter was a photon detector used during dedicated normalization runs, to measure the tagging efficiency of the tagger hodoscope Tcounters. Its detection efficiency was almost 100\%. The Shower Counter, placed downstream of CLAS, had four lead-glass blocks coupled to a Photo-multiplier. Special runs operated with a $100 \mathrm{pA}$ electron beam and a thinner radiator to produce fewer photons than used during production runs. The ration of photons detected in the shower counter to "good" electrons provided the inverse of the tagging efficiency, necessary to determine the photon flux for the experiment. "Good" electrons were then correctly selected (in time) by the tagger system, as described in section 2.3.7.

\section{Circularly polarized photons}

Circularly-polarized photons of the g14 run period were produced via Bremsstrahlung reaction of longitudinally polarized electrons from a thin gold foil radiator of thickness $10^{-4} X_{0}$, where $X_{0}$ is the gold radiation length $\left(6.46 \mathrm{gm}^{-2}\right.$ or $\left.0.3344 \mathrm{~cm}\right)$. Gold was chosen because of its high atomic number $(Z=79)$, which resulted in a reduction of the background from electron-electron scattering in Bremsstrahlung reaction. The photons produced via a Bremsstrahlung reaction will have a degree of circular polarization proportional to the longitudinal polarization of the electron beam, according to the formula [31]: 


$$
P_{\odot}=P_{e} \frac{4 x-x^{2}}{4-4 x+3 x^{2}}
$$

where $P_{e}$ is the degree of longitudinal polarization of the electron beam calculated as in 2.1 and

$$
x=\frac{E_{\gamma}}{E_{e}}
$$

corresponds to the fraction of electron energy carried by the photon. In Figure 2.3 the degree of circular polarization of the photon beam as a function of the variable $\mathrm{x}$ it is shown; it can be seen that it is almost $100 \%$ when the photons carry off the maximum available energy, but it decreases as the fraction of the available energy given to the photon reduces. Figure 2.4 shows the Bremsstrahlung photon energy spectrum from one of the target period of g14 data, (see chapter 4).

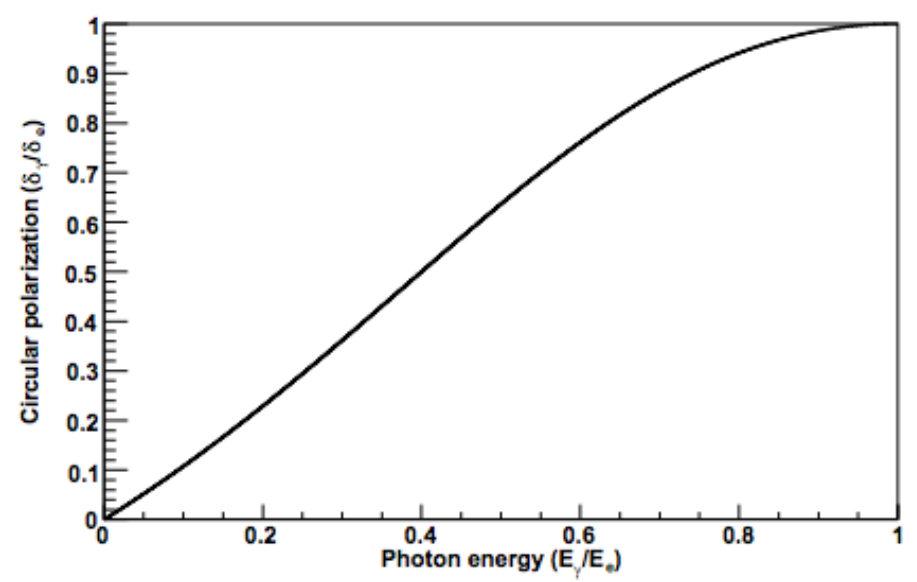

Figure 2.3: Degree of polarization as a function of the fraction of the available energy carried by the Bremsstrahlung photon.

\section{Linearly polarized photons}

Linearly polarized photons used in the g14 experiment were produced using coherent Bremsstrahlung from a thin oriented diamond radiator of $50 \mu \mathrm{m}$ thickness. The diamond was chosen because of its small lattice constant and high Debye temperature $(2200 \mathrm{~K})$ [32], [33], which result in a reduced thermal motion of the atoms in the lattice. When the diamond lattice is aligned relative to the electron beam direction, the photons produced via the remsstrahlung reaction will have discrete fraction energies, 


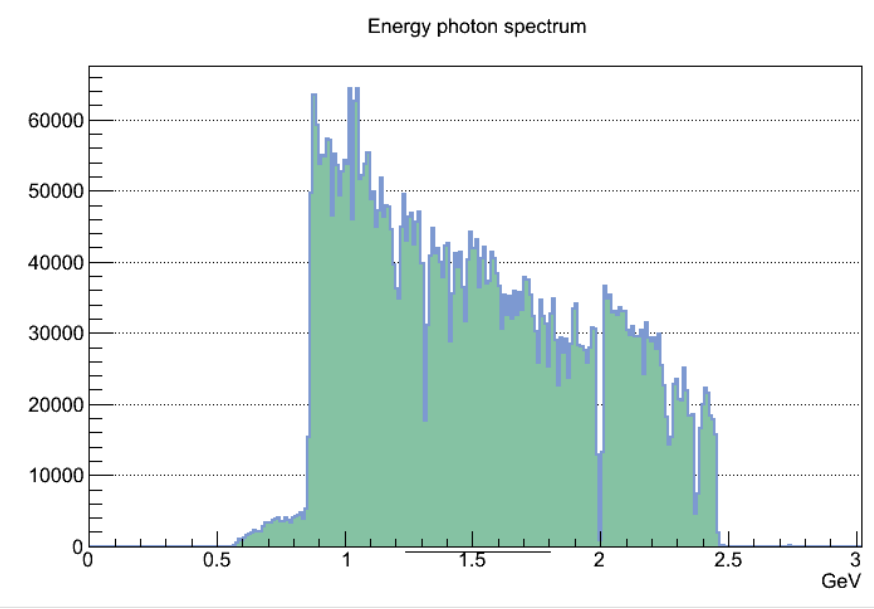

Figure 2.4: The tagged photon energy spectrum for circularly polarized photon beam from the "gold2" (see table reftab1 of ) period of g14 data. The corresponding average photon polarization is $P_{\gamma}=88 \%$.

corresponding to specific momentum transfers $\vec{q}$ of the electrons to the crystal nuclei, according to the Laue condition $\vec{q}=\vec{g}$, where $\vec{g}$ is the reciprocal-lattice vector of the crystal; this will result in sharp coherent peak structures at the energies that correspond to the crystal lattice orientations, as shown in Figure 2.5. The produced photons, in contrast to the spectra associated with incoherent Bremsstrahlung, will have a high degree of linear polarization in the coherent peak regions [34]. Since the coherent photons were produced by momentum transfer along the fixed direction of the reciprocal lattice vector, the photon polarization vector will lie predominantly in a single plane resulting in linearly polarized photons at that energy. It has been found that the highest photon polarization is achieved by scattering from the planes described by the [022] and [022] reciprocal lattice vectors. An enhancement in the scattering, however, occurs not only from the primary reciprocal lattice vector $([02 \overline{2}]$ in this case) but to a gradually decreasing extent also from higher order planes such

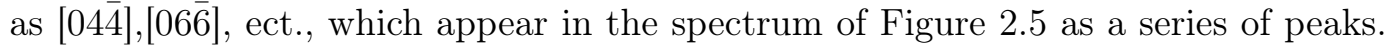
The total photon spectrum, shown in the lower figure, has been divided by the one obtained using an amorphous radiator to remove the characteristic Bremsstrahlung intensity dependence.

In order to control the alignment of the crystal relative to the electron beam, the diamond radiator was mounted on a goniometer $(44 \mathrm{~cm} \times 20 \mathrm{~cm} \times 76 \mathrm{~cm})$ [35]. This device allowed to move the diamond in horizontal and vertical directions and rotate it around all three axes with high accuracy. The goniometer was located $22 \mathrm{~m}$ upstream the tagger magnetic spectrometer inside vacuum chambers and it is shown in Figure 2.6. 

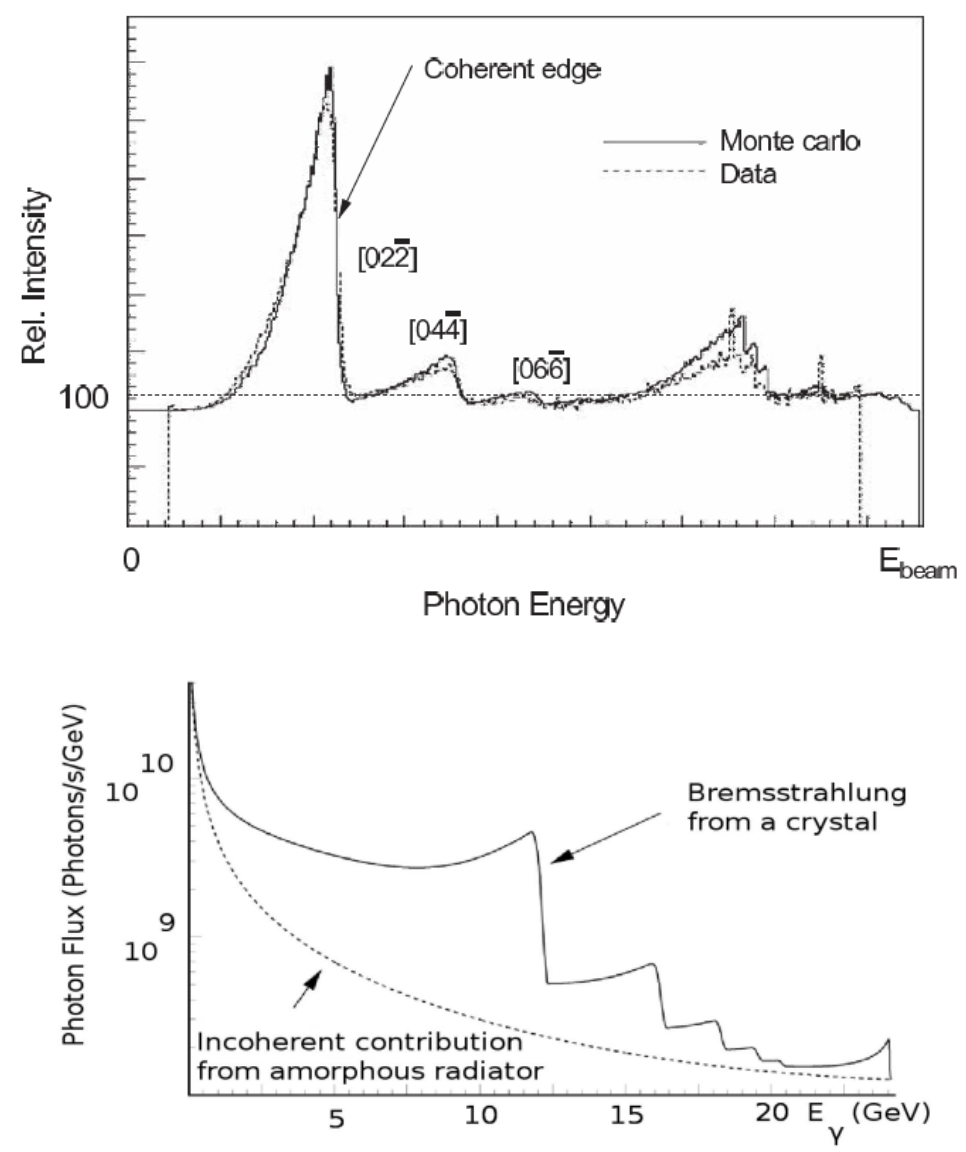

Figure 2.5: Upper Figure: data and Monte Carlo simulation of the photon intensity spectrum produced by the coherent Bremsstrahlung [35] normalized to the incoherent spectrum from an amorphous radiator. Lower Figure: dashed line represent the incoherent Bremsstrahlung contribution from an amorphous radiator. The continuous line shows coherent Bremsstrahlung from a diamond crystal [36]. 


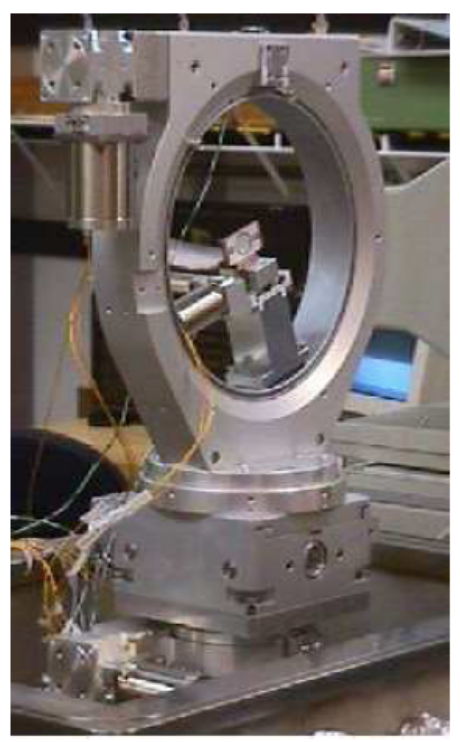

Figure 2.6: The Hall B goniometer. Diamond radiators are mounted on the target ladder centered in the cradle [30].

\subsubsection{Photon Beam Collimation}

The photon beam collimators were placed downstream of the tagger magnet to allow for removal of the photon beam halo.

The choice of the width of the collimator strictly depends on the type of photon polarization used. Linearly polarized photons produced via coherent Bremsstrahlung possess an emission angle relative to the incident beam smaller than for incoherent Bremsstrahlung, being this latter undesired background. It follows that, in order to increase the degree of linear polarization, the best choice is using collimators with small width, which will significantly reduce the contribution from incoherent background. However, at the same time, the small width of the collimator could result in a significantly reduced photon transmission, making this type of collimator appropriate for determination of polarization observables like photon beam asymmetry, but not of cross sections. For this reason during the g14 data taking with linearly polarized beam two collimator $2.0 \mathrm{~mm}$ wide were used, made from nickel diskettes with a small hole in their center; they were placed in a cylindrical sheath of stainless steel with four $4 \mathrm{~mm}$ diametric cubic scintillator between the first two diskettes to measure the rate of off-centered photons hitting the front face of the collimators. As for circularly polarized photons, they are emitted isotropically in the $\phi$ angle describing, a cone-like shape around the incident beam axis such that, when reaching the target the photon beam, can become size variable relative to the target width. 
The number of photons hitting the target will be large but there can be background related to interactions with the target walls, so the collimator width in this case must be chosen as a compromise between the photon flux and the background; this kind of collimator will be suitable for high-quality cross sections measurements. During the g14 data taking with circularly polarized beam, a collimator $2.6 \mathrm{~mm}$ wide was used. Interspaced sweep magnets permitted to clean up any charged particle background generated in the collimator walls.

\subsubsection{The photon tagger}

The photon-tagging system allowed for the investigation of real photon-induced reactions and was based upon the Bremsstrahlung tagging technique for event by event measurement of incident photon energy in photo-nuclear interaction. The geometry of the system is sketched in Figure 2.7.

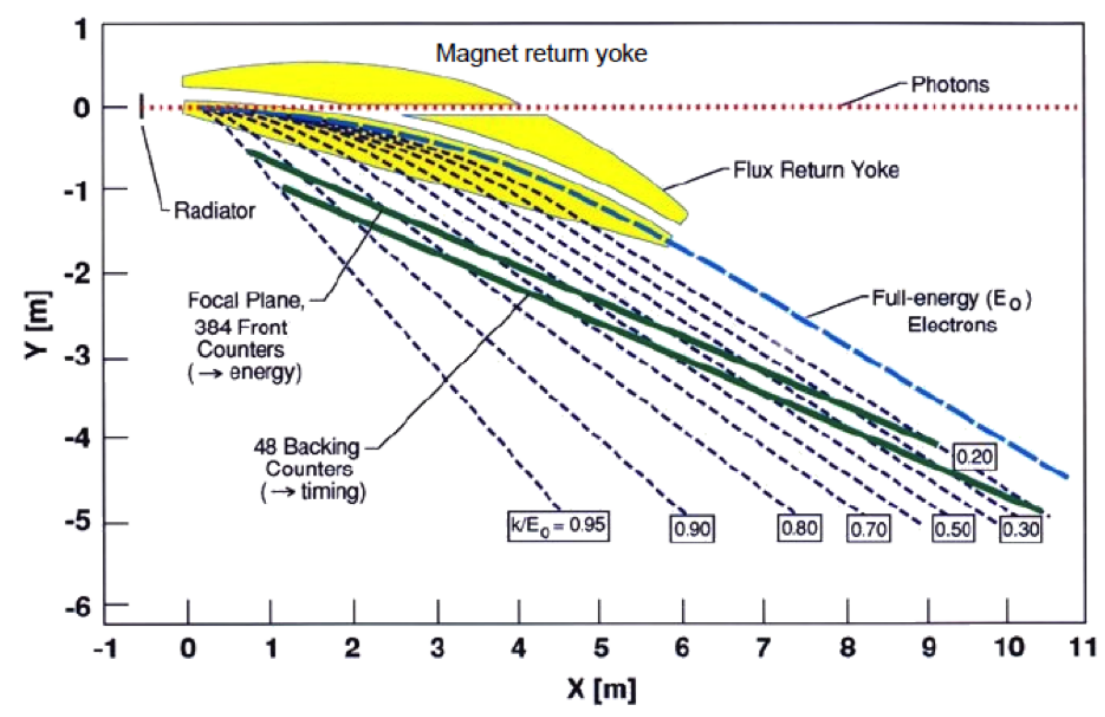

Figure 2.7: The tagging spectrometer sketched in cross-section, showing the photon beam, the paths of the scattered electrons deflected in the tagger magnetic field and the two planes of the scintillator hodoscope [37].

Electrons from the CEBAF accelerator struck the radiator (a gold foil or a diamond crystal, depending on which type of polarization is used during the data taking, see sections 2.3.5 and 2.3.5) and produced photons via Bremsstrahlung reaction. Once the electron has radiated a photon, it lost part of its energy; since the energy transferred to the nucleus in negligible, the associated photon energy can be calculated as: 


$$
E_{\gamma}=E_{0}-E_{e}
$$

where $E_{0}$ is the incoming electron beam energy, determined only by the CEBAF accelerator parameters, $E_{e}$ is the energy of the outgoing electron and $E_{\gamma}$ is the energy of the emitted photon. The angular distribution of the photons is contained within a characteristic angle:

$$
\theta_{c}=\frac{1}{\gamma}=\frac{m c^{2}}{E_{0}}
$$

where $\mathrm{m}$ is the electron rest mass. The electron scattering angle is a function of the photon scattered angle and the electron energies, as follows:

$$
\theta_{e}=\theta_{c} \frac{E_{\gamma}}{E_{e}}=\frac{m c^{2} E_{\gamma}}{E_{0} E_{e}}
$$

At JLab energies $(>800 \mathrm{MeV})$ both of these angles are $\leq 1 \mathrm{mr}$, so in first approximation both the photons and the electrons are still traveling along the original beam direction. Any photons produced in the interaction with the radiator thus continued straight ahead through the tagger magnet, toward the target farther downstream in the experimental Hall. Downstream the target a collimation system was placed to further define the photon beam (see section 2.3.6). The field setting of the magnet was matched to the incident beam energy so that the trajectory of those electrons which didn't radiate losing their energy was a circular arc, just inside the curved edge of the pole face, directed into a shielded beam dump below the floor of the experimental hall. Electrons which emitted a photon, instead, experienced greater curvature in the tagger field, and merged from the magnet somewhere along the straight edge of the pole gap. A scintillator hodoscope along the flat focal plane, downstream from this straight edge, detected these electrons which had between $5 \%$ to $80 \%$ of the maximum energy $E_{0}$, and allowed for the determination of the energy of the radiated photons.

The hodoscope had two planes: the upper one referred to as E-plane, was equipped with 384 plastic scintillator paddles that were $20 \mathrm{~cm}$ in length, $4 \mathrm{~mm}$ thick and widths between 6 and $18 \mathrm{~mm}$, in order to cover approximately the same energy fraction of the incident electron beam. Each scintillator paddle, referred to as E-counter, was readout via a photomultiplier tube (PMT) and connected to a multi-hit TDC. The E-counters were arranged to be partially overlapping to create 767 E-channels, each providing an energy resolution of $0.001 E_{0}$ per bin. The lower plane of the hodoscope, referred to as T-plane, was instrumented with 61 scintillator paddles, overlapped by about $10 \%$ of their width, forming 121 T-channels. Each T-counter had a doublesided PMT readout, with each PMT connected to a discriminator and a pipeline TDC. The T-counters were $2 \mathrm{~cm}$ thick and had a timing resolution of $110 \mathrm{ps}$. This 
good timing resolution permitted to identify the correct beam bucket to which associate every photon. The RF time for that bucket may then be correlated with a hadron detected in CLAS to calculate the correct event vertex time. Further details about the photon tagger system can be found in Ref [37].

\subsection{The HD frozen spin polarized target}

The HD target was a solid state target made of $99 \%$ of pure HD molecules, where both proton and deuteron were polarized. The HD target was polarized by the "brute force method" [44] and it required about 3 months to reach the "Frozen Spin" mode necessary to acquire a polarization lifetime long enough to perform the g14 experiment. It was placed inside CLAS at an offset of $-7.5 \mathrm{~cm}$ with respect to the CLAS centre. The target, along with the method used to polarize it, will be described in detail in chapter 3.

\subsection{The CLAS detector}

The CLAS (CEBAF Large Acceptance Spectrometer), housed in Hall B, was a nearly $4 \pi$ detector designed for studying the electromagnetic interactions with nucleons and nuclei. The CLAS detector inside of Hall B is shown in Figures 2.8 and 2.9. Further details may be found in Ref. [38]. The particle detection system used for the HDice experiment consisted of:

- Start Counter

- Superconducting Toroidal Magnet

- Drift Chambers to determine charged particles trajectories

- Scintillation counters for time-of-flight (TOF) measurement

- Electromagnetic calorimeter to identify showering particles such as electrons and photons.

The various component of the detectors will be described in the next sections.

\subsubsection{Start counter}

The start counter surrounding the HD target was the first detector encountered by a particle produced in a photo-reaction with the HD target. It provided the most accurate timing information for the event; in particular its information was crucial to synchronize the particle detected by CLAS with the tagged beam that started the reaction. 


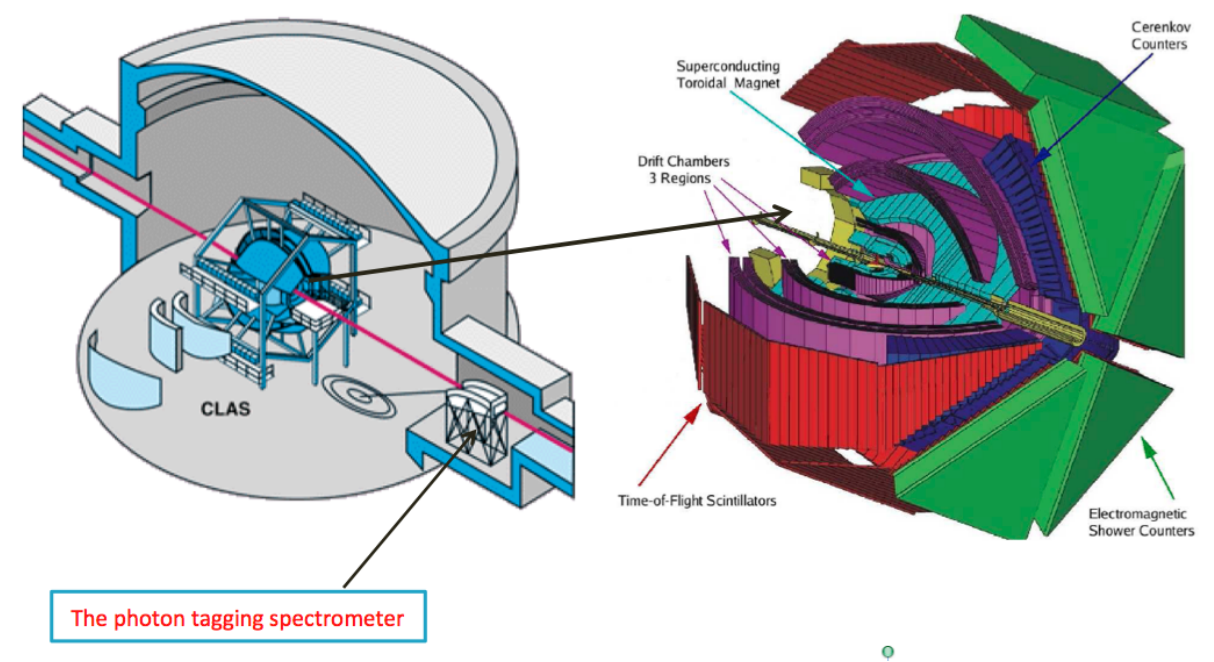

Figure 2.8: Schematic view of the CLAS detector along with the beam line and the photon tagging spectrometer [38].
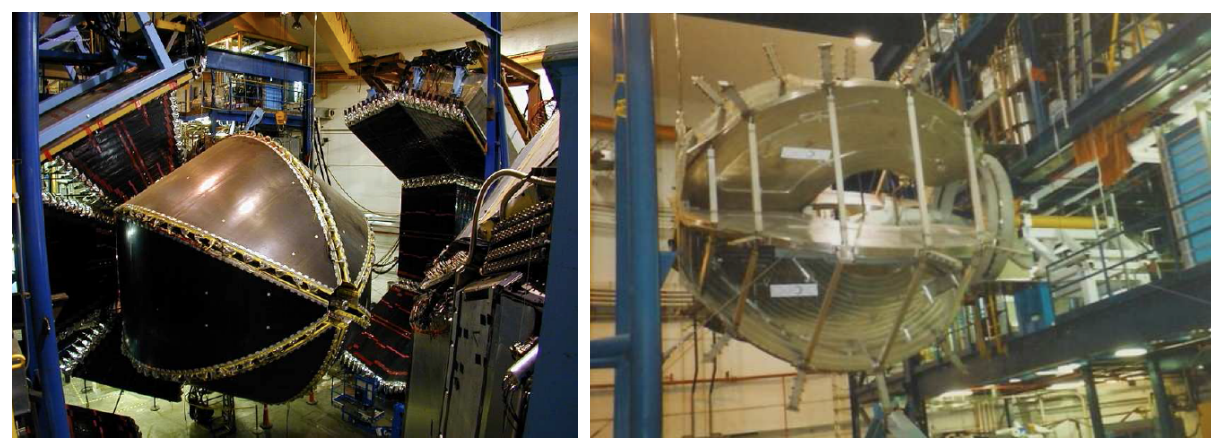

Figure 2.9: Left: the inside of Hall B; the detector CLAS was open for servicing [30]. Right: the CLAS torus coils before the detector installation [30]. 
It was structured in 6 sectors arranged azimuthally around the target, each consisting of four EJ-200 organic plastic scintillators paddles with acrylic light guides. Each paddle had a straight rectangular section (leg) with a length of $502 \mathrm{~mm}$ and a triangular nose region of $93 \mathrm{~mm}$, as shown in Figure 2.10. They were $2.15 \mathrm{~mm}$ thick and $29 \mathrm{~mm}$ wide; their thickness was chosen in order to minimize multiple scattering effects. This setup allowed for a coverage of the full acceptance of CLAS, from $7^{\circ}<\theta<145^{\circ}$ and $-29^{\circ}<\phi<29^{\circ}$ for each sector.

In order to provide light isolation, the paddles were wrapped in radiant mirror film (VM-2000), with alternate ones being additionally covered in black Tedlar film. At the upstream end of each paddle, a $127 \mathrm{~mm}$ long acrylic guide coupled the scintillator to a Hamamatsu 10-stage R4125Ha photomultiplier tube, $15 \mathrm{~mm}$ in diameter. The PMT signals were digitized by LeCroy FASTBUS time-to-digital converters (TDCs) and analogue-to-digital converters (ADCs) before being read out into the data stream. The timing resolution of each paddle depended strictly on the region, varying from $292 \mathrm{ps}$, in the leg region, to $324 \mathrm{ps}$ in the nose region. Further details about the start counter can be found in Ref. [40].

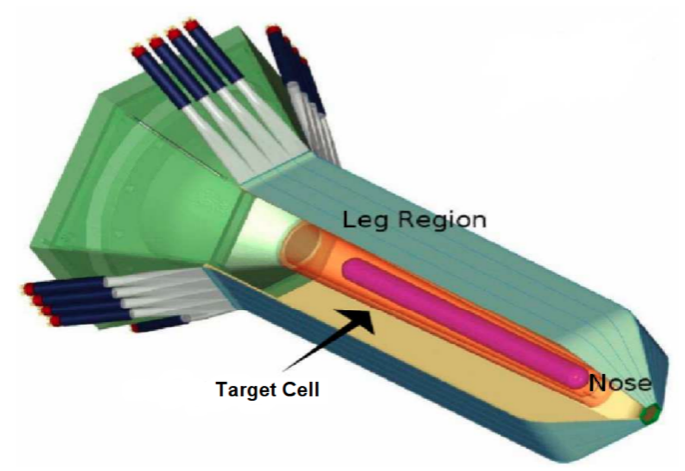

Figure 2.10: Schematic view of the Start Counter.

\subsubsection{Superconducting Toroidal Magnet}

The magnetic field of CLAS was generated by six superconductor coils arranged in a toroidal geometry around the beam line; it bended charged particles passing through the drift chambers allowing for momentum measurements, since the particles path was recorded by the drift chamber system and this information was used to fit the trajectory and momentum of each particle.

The magnet was $5 \mathrm{~m}$ in diameter and $5 \mathrm{~m}$ in length. For a fixed target experiment the highest momentum particles are generally emitted at forward angles, in order to provide optimum degrees of curvature in the tracks of charged particles, the coils were 
designed to have a kidney-shape (see Fig. 2.11) allowing to have a high magnetic field at forward angles and lower field at larger angles, where particle momentum are typically smaller. At the same time, this geometry preserved a central field-free volume allowing for the use of a polarized target and causing negligible deflections to a charged beam. The only drawback was that the width of the coils and cryostats reduced the CLAS acceptance to about $80 \%$ of the $4 \pi$ solid angle.

Each coil had four layers of 54 turns of aluminum-stabilized niobium-titanium/copper $(\mathrm{NbTi} / \mathrm{Cu})$ wire and was fixed to a $4.5 \mathrm{~K}$ super-critical He cooling cryostat.

At the maximum designed current of $3860 \mathrm{~A}$, the magnetic field could reach value of $2.5 \mathrm{~T} \cdot \mathrm{m}$ in the forward regions and drop to $0.6 \mathrm{~T} \cdot \mathrm{m}$ at a scattering angle of $90^{\circ}$, and was concentrated mostly in central region of the Drift Chambers. During the g14 running period the magnetic field alternated positive and negative polarities by passing a current of $1918 \mathrm{~A}$ and -1495 A, respectively. All PMT's from electromagnetic calorimeter and the time-of-flight system, as well as as the Drift Chambers supports system, were located in correspondence to the shadow of the torus coils maximizing the angular acceptance. Further information about the torus can be found in Ref. [39].

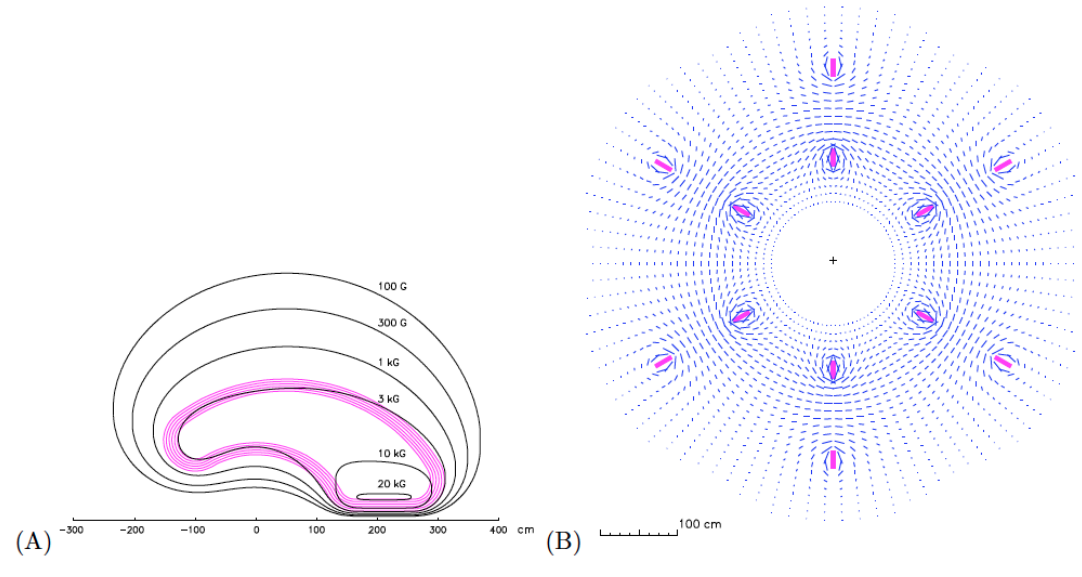

Figure 2.11: Left: Contours of the magnetic field generated by a kidney-shaped torus in the mid plane between two coils. Right: Magnetic field vectors for the CLAS torus transverse to the beam in a plane centered on the target [38].

\subsubsection{Drift chambers}

The determination of the momentum of charged particles was performed by tracking the particles as they travelled through the field generated by the torus magnet.

There were 18 drift chambers arranged concentrically in three regions around the 
target. Each region, referred to as Region 1, 2 and 3, respectively, consisted of six drift chambers located at the same radius in the six sector of CLAS. Region 1 was located inside the torus coil, just outside the Start Counter, where the magnetic field was weaker. Region 2 was mounted directly to the magnet's cryostat, where the magnetic field was the strongest. Region 3 was located instead outside the torus coils, where, again, there was a weak magnetic field. The relative position of the three regions can be seen in Figure 2.12.

Each drift chamber was divided into two super layers, one with axially orientated wires (relative to the magnetic field direction) and one with wires orientated at 6 stereo angle. Each super layer consisted of six layers of hexagonal drift cells arranged such that neighboring layers were offset by a half cell width. Each cell had a $20 \mu \mathrm{m}$ gold-plated tungsten sense wire located at its centre, surrounded by six $140 \mu \mathrm{m}$ goldplated aluminum alloy field wires. The sense wire possessed a positive potential, while the field wires were kept at negative high voltage. Signals from the sense wire were passed to preamplifiers, then to amplifier discriminator boards (ADBs) and finally to TDC's to obtain timing informations.

The drift chambers contained a mixture of $90 \%$ Argon $10 \% \mathrm{CO}_{2}$, chosen because of its ionization properties and because it's non-flammable. More information can be founded in [41].
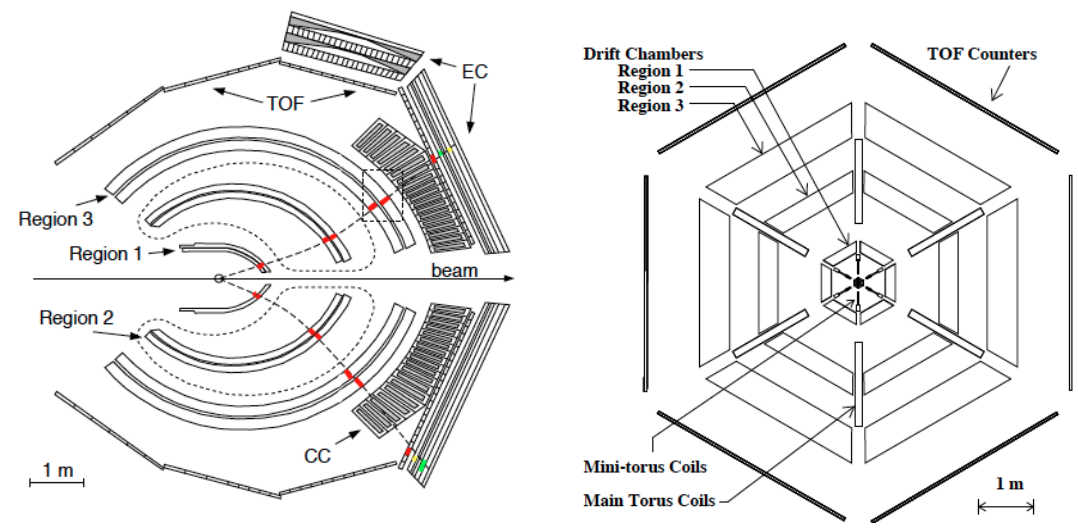

Figure 2.12: Left: schematic view of the CLAS detector showing the position of the three Drift Chambers region. Tracking reconstruction for positively and negatively charged particle is shown. Right: schematic view of the CLAS six sectors showing the Torus coils positions [38]. 


\subsubsection{Time-of-flight scintillators}

After passing through the drift chambers, particles hit the time-of-flight detector (ToF). The time-of-flight detector measured the flight time of particles from the interaction vertex in the target to the ToF counters. This timing information, along with tracking information from the Drift Chambers, was crucial to perform charged particle identification.

The ToF system was arranged in six sectors, each one equipped with double-ended scintillators placed between the Cherenkov counters and the forward electromagnetic calorimeter. It covered the CLAS detector in the entire azimuthal region, while the polar angle varied between $8^{\circ}$ and $142^{\circ}$. A schematic of one of the sectors is shown in Figure 2.13.

The scintillator wall in each sector was made up of 57 scintillator paddles, fabricated from Bicron BC-408 material, of varying lengths and widths. Most of the produced tracks travel through the forward region of CLAS, at angles $<45^{\circ}$ from the beamline, where the scintillators where $15 \mathrm{~cm}$ wide. At angles greater than $45^{\circ}$ the scintillator bars were $22 \mathrm{~cm}$ wide. The length varied across the azimuth of the detector from 32 to $445 \mathrm{~cm}$. The thickness of the scintillators was instead fixed to $5.1 \mathrm{~cm}$, allowing to maximize the detection efficiency of minimum ionizing particles.

The signal from the scintillator were collected and amplified by photomultiplier tubes (PMTs) attached at each end; the outputs were fed to a Fan In/ Fan Out and then to both ADC and TDC boards. The output from the TDC board is an important part of the Level 1 trigger and, used in conjunction with the time information from the Start Counter: it allowed to calculate the $\beta$ of charged particles. The timing resolution of each scintillator was between 60 and 160 ps for the forward counters and between 100-160 for the large angle counters. More information may be found in [42].

\subsubsection{Forward electromagnetic calorimeter}

The forward electromagnetic calorimeter was a sampling calorimeter made of alternating layers of scintillator strips and lead sheets with a total thickness of 16 radiation length $X_{0}$. A lead:scintillator thickness ratio of 0.2 was used, requiring 40 $\mathrm{cm}$ of scintillator and $8 \mathrm{~cm}$ of lead per module. With this ratio approximately $1 / 3$ of the energy in a shower is deposited in the scintillator.

In order to match the hexagonal geometry of the CLAS apparatus, the lead-scintillator sandwich was contained within a volume having the shape of an equilateral triangle, as shown in Figure 2.14. There were 39 layers in the sandwich, each one consisting of a $10 \mathrm{~mm}$ thick BC412 scintillator, followed by a $2.2 \mathrm{~mm}$ thick lead sheet.

The calorimeter design was based on a projective geometry, according to which the area of each successive layer increases; this design allowed to minimize shower leakage at the edge of the active volume and minimize the dispersion in arrival times of 


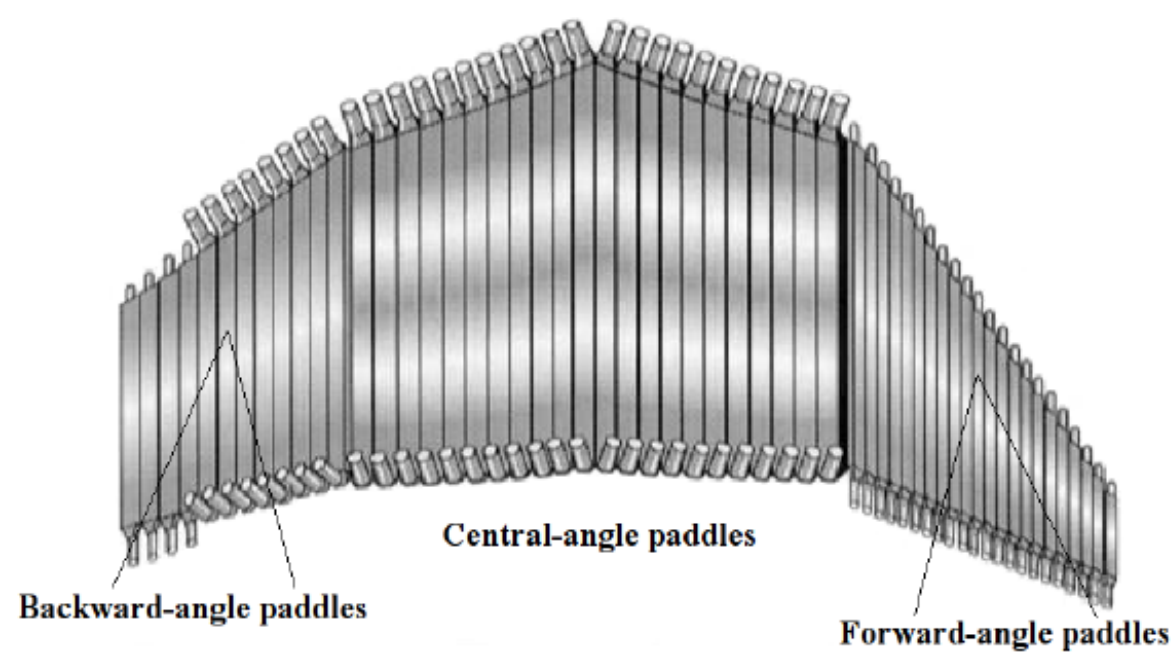

Figure 2.13: Schematic view of one of the sector of the Time-of-Flight system. It shows the 56 paddles divided into backward angles (12 paddles), central angles (22 paddles), and forward angles (22 paddles) [42].

the signals originating in different scintillator layers.

The active volume of the sandwich formed a truncated triangular pyramid with a projected vertex at the CLAS target point, $5 \mathrm{~m}$ away, and with an area at the base of $8 \mathrm{~m}$.

Each scintillator was made up of 36 strips parallel to one side of the triangle and the orientation of the strips was rotated by 120 in each successive layer. This defined three orientation or views, U,V and Z, each containing 13 layers, providing thus stereo information on the location of energy deposited. Each view was divided into a inner (5 layers) and outer (8 layers) Stack, to provide longitudinal sampling of the shower for improved hadron identification. There were a total of 6 modules, meaning that there were a total of 1296 strips (36 (strips) x 3 (views) x 2 (stack) $\mathrm{x}$ 6 (modules)).

A fiber optic readout system was used to transmit the scintillation light to PMTs. The PMTs and the light guide adapter were coupled using optical grease, while the light guide adapter was glued to the fiber readout bundle with a UV curing compound. Other information may be found in Ref. [43]. 


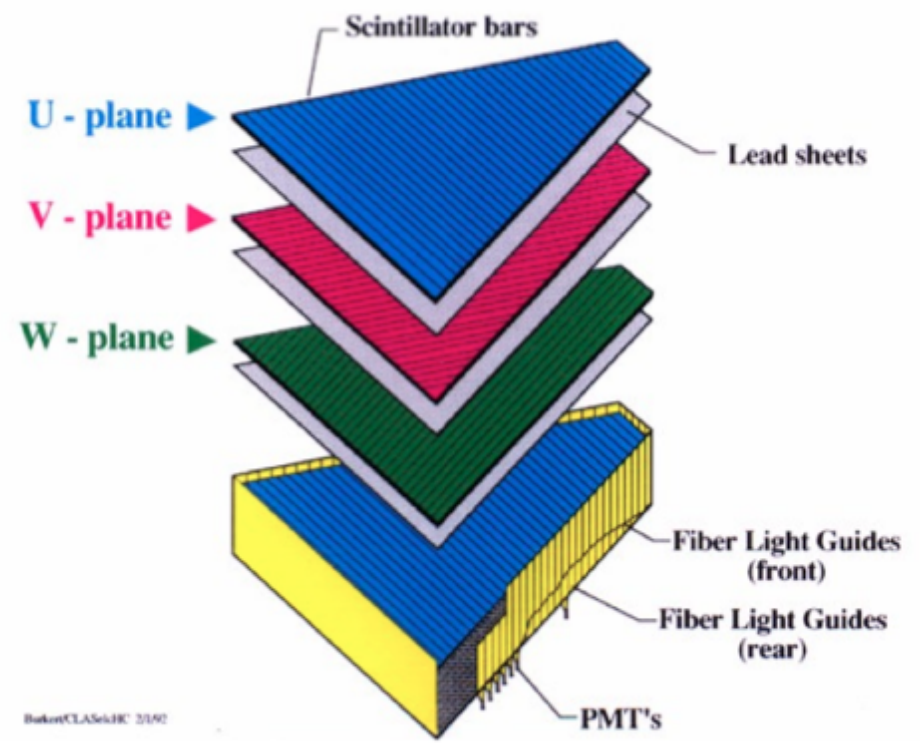

Figure 2.14: One of the six modules of the CLAS Forward Electromagnetic Calorimeter [43]. 


\section{Chapter 3}

\section{The HD-ice target}

\subsection{Introduction}

The target used for the g14 experiment was a Hydrogen-Deuteride (HD) polarized target; it was made of HD molecules in the solid state including $\mathrm{H}_{2}$ and $\mathrm{D}_{2}$ impurities, whose presence was essential for polarizing the target, as we will see in section 3.3 .

The HDice target offered the possibility to investigate at the same time many photoproduction reaction channels on both neutron and proton targets. Another peculiar feature of the HDice target was that it contained only protons and deuterons which can both polarized with a high degree of polarization $(90 \%$ for $\mathrm{H}$ and $60 \%$ for $\mathrm{D}$, in principle). Other type of targets, for example ammonia targets $\left(\mathrm{NH}_{3}\right.$ or $\left.\mathrm{ND}_{3}\right)$, contain instead additional non-polarizable nuclei which constitute a main background source. Another very attractive property of the HD target is that it was polarized in a "frozen spin mode", which means that its polarization can be kept for months under experimental conditions $(\mathrm{B}=1 \mathrm{~T}$ and $\mathrm{T}<500 \mathrm{mK})$.

The price to pay is that the target handling procedures are very complex and that the production of a polarized HD target in frozen spin mode requires at least three months.

This chapter is organized as follows: in sections 3.2 and 3.3 the target geometry and the physics concepts behind it, respectively, will be described. Section 3.4 is dedicated to the Raman spectroscopy technique we used for target characterization purpose. In section 3.6 the equipment of the HD-ice target is described; in section 3.7 we will give a description of the typical sequence used to produce a frozen-spin polarized HD target and transport it to an In-Beam Cryostat located in the experimental hall. 


\subsection{HD-ice Target geometry}

In Figure 3.1 a typical target cell is shown, together with a view of its components. The target cell had approximately a cylindrical shape and consisted of an outer shell, an inner cell, aluminum wires and a copper ring. The shells (or walls) were made of a fluoropolymer, PCTFE (PolyChloroTriFluoroEthylene, $\mathrm{C}_{2} \mathrm{ClF}_{3}$ ) known as Kel-F, a hydrogen-free polymer that does not add background to the $\mathrm{H}$ or $\mathrm{D}$ signals measured with the Nuclear Magnetic Resonance (NMR). Other interesting properties of Kel-F at very low temperatures $(\mathrm{T}=10 \mathrm{mK})$ are: low permeation, low deformation and good mechanical, chemical and radiation resistance. The side walls of the Kel-F cylinders were $0.5 \mathrm{~mm}$ thick, while the faces through which the beam passed were thinned to $0.1 \mathrm{~mm}$. The shells were glued on the copper ring and, inside its cylindrical part, the outer cell was filled with HD that, once solidified, took the form of a $50 \mathrm{~mm}$ long and $15 \mathrm{~mm}$ diameter cylinder.

The downstream face of the copper ring had 60 holes, into which 36 bundles of aluminum cooling wires $(99,9998 \% \mathrm{Al})$ of $50 \mu \mathrm{m}$ diameter were soldered, for a total of 750 wires. One of the bundle it's shown in Figure 3.1. The aluminum wires were placed in the target cell to enhance the heat conductivity. They allowed to conduct out of the HD crystal the heat generated in the polarization process, as well as the heat produced by beam interaction or energy level transitions. As part of the wires are in the beam trajectory, the Aluminum was a good choice to reduce background from event production with the beam, still guarantying thermal conductivity, because of its low density and low mass numbers.

The target's copper cooling ring had right-hand threads along its outer surface and left-hand threads along it's inner bore. These were used in the process of moving the target between dewars. The outer threads maintained thermal contact with any of the cryostats that held the target in the production and transfer phases. The threads along the inner bore allowed for the HD target extraction during transfer operation. These operations will be explained in detail in section 3.6. 

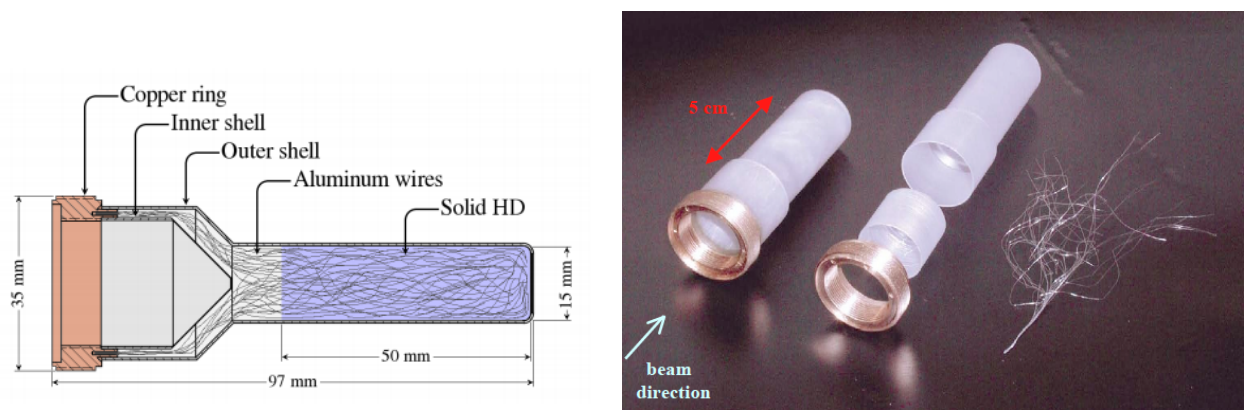

Figure 3.1: Typical HD target used during the E06-101 (HD-ice) experiment at JLab. A shell of pCTFE is attached to a copper target ring that has an interior lefthanded thread (engaged by tools to move the target cell) and exterior right-handed thread (used to mount the target in a dewar). Several hundred thin aluminum wires span the length of the target cell and are soldered at one end into the target ring. A $50 \mathrm{~mm}$ long cylindrical crystal of HD is grown in the target cell by condensing and then freezing distilled HD gas. The embedded aluminum cooling wires allow heat, generated in the HD crystal during the polarization process and photo nuclear interaction, to escape from the target and into the mixing chamber of a dilution fridge.

\subsection{HD-ice Target physics}

The active material of the g14 experiment was composed by solid Hydrogen-Deuteride (HD). The specific choice of the ethero-nuclear HD molecule can be understood considering the symmetry constraints that the homo-nuclear $H_{2}$ and $D_{2}$ molecules must obey to. In fact, a $\mathrm{H}_{2}$ molecule consists of two identical protons, which are fermions; it follows that the molecular wave-function must be anti-symmetric under the exchange of two identical nuclei. On the contrary, $D_{2}$ contains two deuterons composed of 1 proton and 1 neutron, so the molecular wave-function must be symmetric under the exchange of spin 1 nuclei. In the Born-Oppenheimer approximation the molecular wave-function can be written as the following product of wave-functions, that depend on different degrees of freedom:

$$
\Psi_{\text {mol }}=\Psi_{\text {electronic }} \times \Psi_{\text {vibrational }} \times \Psi_{\text {rotational }} \times \Psi_{\text {nuclear }}
$$

The exchange of the two nuclei corresponds to space inversions for electronic and space variables. Therefore the wave-function symmetry is directly related to its parity. The vibrational ground state is symmetric with respect to space inversion and so is also the electronic wave function, since $P=(-1)^{L}$ where $\mathrm{L}$ is the electronic orbital eigen-value. $\Psi_{\text {rotational, }}$, the rotational states, are symmetric for even values of the eigen-values $\mathrm{J}$ and anti-symmetric for odd-values $\left(P=(-1)^{J}\right)$. In the case 
of $\mathrm{H}_{2}, \Psi_{\text {nuclear }}$ is symmetric when the two nuclear spins couple to a total value $\mathrm{I}=1$ (ortho-hydrogen) and anti-symmetric for $\mathrm{I}=0$ (para-hydrogen) and we have that $P_{H_{2}}=(-1)^{J}(-1)^{I+1}$ in the electronic and vibrational ground state. It follows that the wave-function symmetry limits the rotational and nuclear states to have the following combination:

- J odd rotational eigen-values must couple to ortho- $H_{2}$ states

- $\mathrm{J}$ even rotational eigen-values must couple to para- $H_{2}$ states

The $\mathrm{I}=0$ and $\mathrm{J}=0$ being the ground state and $\mathrm{I}=1$ and $\mathrm{J}=1$ the first metastable excited state.

As for $D_{2}$ molecules the nuclear wave-function is symmetric when the two spins couple to $\mathrm{I}=0,2$ (ortho-deuterium) and it is anti-symmetric for $\mathrm{I}=1$ (para-deuterium). In this case $P_{D_{2}}=(-1)^{J}(-1)^{I}$ and we have:

- J odd rotational eigen-values must couple to para- $D_{2}$ states

- $\mathrm{J}$ even rotational eigen-values must couple to orto- $D_{2}$ states

The $\mathrm{I}=0,2 \mathrm{~J}=0$ state being the ground state and $\mathrm{I}=1, \mathrm{~J}=1$ being the metastable excited state. Decays from $\mathrm{I}=1$ and $\mathrm{J}=1$ to $\mathrm{I}=0$ and $\mathrm{J}=0$ are inhibited because two transitions must happen simultaneously in the same molecule: a E1 molecular transition to change the rotational state and a M1 nuclear spin-flip to preserve the symmetry of the wave-function. Among those states the $\mathrm{I}=1$ excited molecular nuclear states are the only polarizable.

As a consequence, homo-nuclear $H_{2}$ and $D_{2}$ molecules cannot be normally used to produce polarized targets.

The HD molecule, instead, has no limitation of symmetry and H and D nuclei may be independently oriented in the molecular ground state.

The maximum degree of polarization that can be obtained at thermal equilibrium is given by the Brillouin function:

$$
P=B_{I}(x)=\left(\frac{2 I+1}{2 I}\right) \operatorname{coth}\left[(2 I+1) \frac{x}{2 I}\right]-\left(\frac{1}{2 I}\right) \operatorname{coth}\left(\frac{x}{2 I}\right)
$$

where:

$$
x=\left(\frac{\mu B}{K_{B} T}\right)
$$

and it depends on the the magnetic field $\mathrm{B}$, the Temperature $\mathrm{T}$, the nuclear magnetic moment $\mu$ and the Boltzmann constant $K_{B}$. For maximum available values of $\mathrm{B}$ and the minimum values of $\mathrm{T}$ obtainable in a magnetic dilution refrigerator $(\mathrm{B}=15$ 


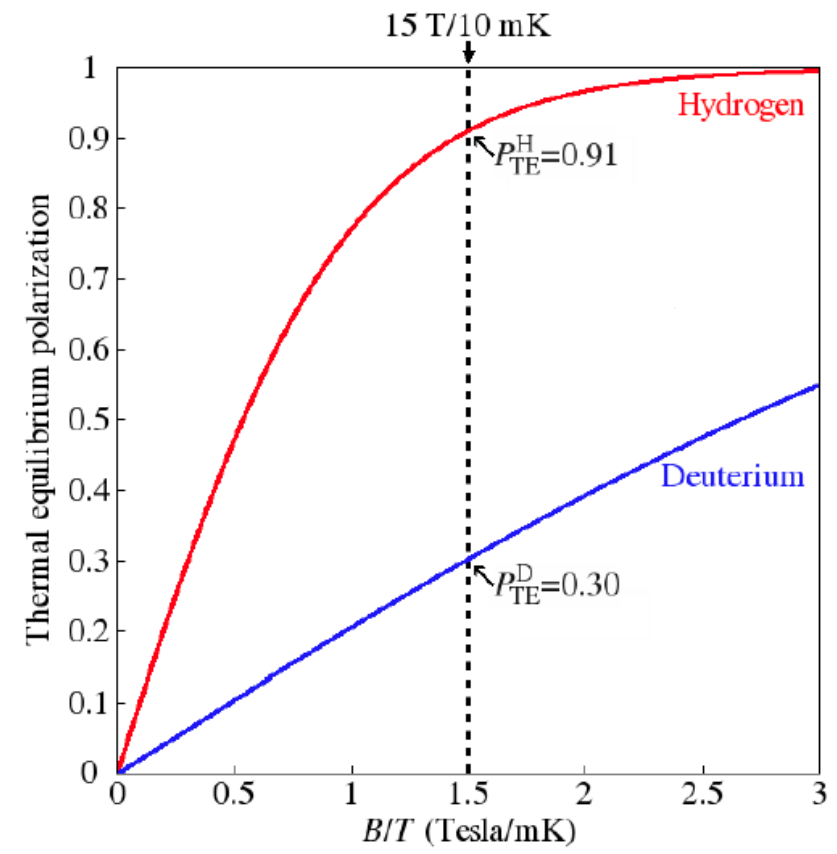

Figure 3.2: Thermal equilibrium polarization (TE) for hydrogen and Deuterium vs $\mathrm{B} / \mathrm{T}$ ratio $[47]$. 
$\mathrm{T}$ and $\mathrm{T}=10 \mathrm{mK}$ ), one reaches maximum values of polarization degrees: $\mathrm{P}=0.91$ for hydrogen and $\mathrm{P}=0.30$ for deuterium, as can be seen in Figure 3.2.

Unfortunately these extreme conditions, called "Brute Force condition" [44], are not compatible with any particle detector of nuclear and sub-nuclear experiments available at the moment.

Besides it was found [53] that, for pure HD solid, direct spin-lattice relaxation mechanisms are suppressed and longitudinal relaxation time for both hydrogen $\left(T_{1}^{H}\right)$ and deuterium $\left(T_{1}^{D}\right)$ in HD solid are extremely long (of the order of several years). However doping the solid HD with small amount of ortho $-H_{2}$ molecules [54], an important relaxation mechanism occurs, due to cross-relaxation of polarized nuclei in $\mathrm{H}_{2}$ with neighbor protons in HD, which have the same Larmor frequency. This effect leads to a faster polarization build-up. The longitudinal relaxation time $T_{1}^{H}$ strongly depends on the concentration of ortho $-H_{2}$ contaminants, being as low as few minutes for ortho $-H_{2}$ concentration of the order of $10^{-3}$ and increases to the order of months for concentration of $10^{-6}$.

Since the energy difference between ortho- and para $-H_{2}$ states is $\Delta T=172 \mathrm{~K}$ (see Figure 3.3), if the HD sample is kept at low temperatures the ortho $-H_{2}$ impurities decay into the para $-H_{2}$ state with a decay time $\tau_{H}=6.3$ days. If we added the initial concentration of ortho $-\mathrm{H}_{2}$ of the order of $10^{-4}$, the relaxation time may be kept short enough to reach the equilibrium polarization value in few days. If, afterwards, the HD is kept at low temperatures and under a high magnetic field (Brute Force condition) for a period of time longer than four times the ortho $-\mathrm{H}_{2}$ decay time (1-2 months), the concentration of impurities will decrease by two orders of magnitude and the relaxation time $T_{1}^{H}$ increases to values of few months. This procedure, based on the spin-lattice relaxation switch, is called "aging" and it allows to achieve a "frozen-spin" mode for the polarized HD target.

In this way we get a short relaxation time during the polarization phase and once the HD is polarized, and after the "aging" period, the target gained a long relaxation time.

In principle, the same procedure could be used to polarize deuterium nuclei by introducing para $-D_{2}$ contaminants in the HD gas, but unfortunately the decay time for para $-D_{2}$ is $\tau_{D}=18.6$ days, resulting in a very long and impractical aging time and the final thermal equilibrium polarization degree would be quite low.

The alternative is to use ortho $-H_{2}$ to build up the proton polarization and then, applying the technique of "adiabatic fast passage", it is possible to transfer the polarization from the hydrogen to the deuteron. The interested reader is referred to reference [55] for further details.

\subsubsection{HD gas distillation}

As it has been stated in the previous section, the polarization procedure strongly depends on the initial concentration of ortho $-\mathrm{H}_{2}$ and para $-\mathrm{D}_{2}$ in the HD gas. 


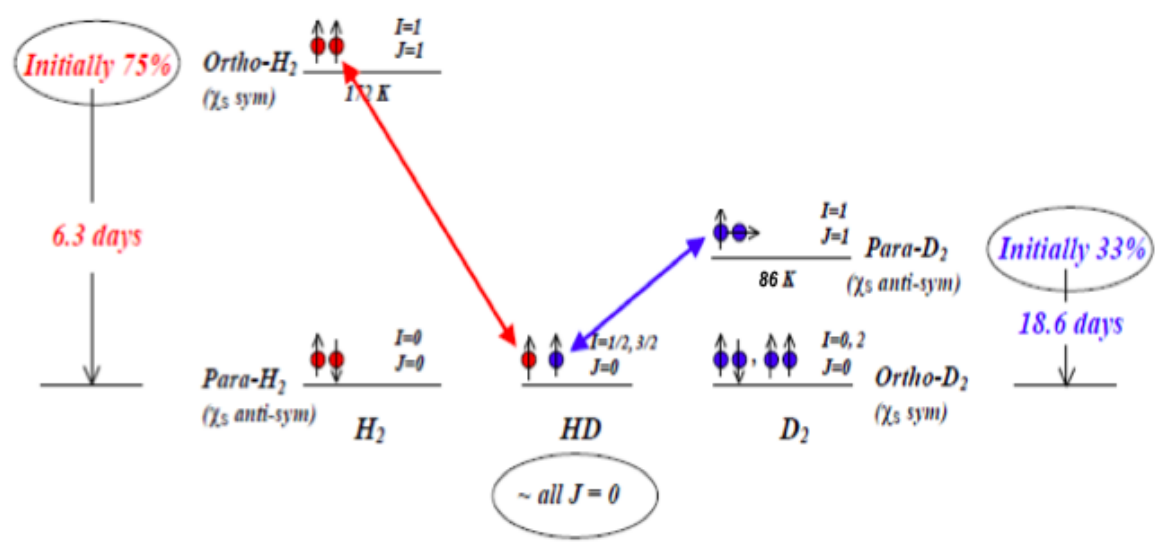

Figure 3.3: Spin exchanges between ortho $-H_{2}\left(\right.$ para $\left.-D_{2}\right)$ and $H(D)$ nuclei in the HD molecule.

The commercial HD gas available is $98 \%$ pure and it contains concentrations of $\mathrm{H}_{2}$ and $D_{2}$ contaminants of the order of $1.5 \%$ and $<0.5 \%$, respectively. The optimal concentrations to achieve a frozen-spin target must be two order of magnitudes smaller. For this aim, a distillation procedure has been developed at the James Madison University (JMU) [49].

The purification procedure is based on the fact that at low temperatures the vapor pressures of $H_{2}, D_{2}$ and $\mathrm{HD}$ are different. The distiller operates at $\mathrm{T}=20 \mathrm{~K}$; it is cooled by a Gifford-McMahon refrigerator and a temperature gradient is set along the distiller tube between the lower still pot, connected to a boil-up heater, and the upper cold finger. A Stedman packaging, consisting in 30 double layers stainless steel mesh located along the distillation tube, amplifies the vapor pressure difference among the different hydrogen isotopes and enables the purification of contaminants by one order of magnitude for each distillation cycle.

The gas is distilled in batches of 12 moles. When the system reaches a steady state, the gas stratifies in the distiller column and can be extracted at a rate of 1 mole/day. The first 3 extracted moles consist of $H_{2}$ enriched HD gas, 6 moles of purified HD gas follow, while the 3 moles remaining in the still pot consist of $D_{2}$ enriched HD gas. The purified gas is then stored in tanks each containing 2 moles of gas. A double-distillation process is required to reduce contaminants to the optimal level of few hundred parts per million.

A residual gas analyzer (RGA) completes the system, which serves to monitor the gas. It uses an electric quadrupole field to momentum analyze ionized particles flowing at a fixed velocity, to determine their mass. Since for hydrogen isotopes the molecular dissociation energy is lower than ionization energy, introducing pure HD 
gas in the RGA results in some recombination of dissociated $\mathrm{H}$ and $\mathrm{D}$ atoms into $\mathrm{H}_{2}$ and $D_{2}$, and a small fraction of $H_{2}$ and $D_{2}$ is always observed.

The sensitivity of this device is limited to some percents, so it can be effectively used only to monitor the extraction of the first $\mathrm{H}_{2}$ enriched moles of HD gas.

A schematic of the distillery is provided in Figure 3.4.

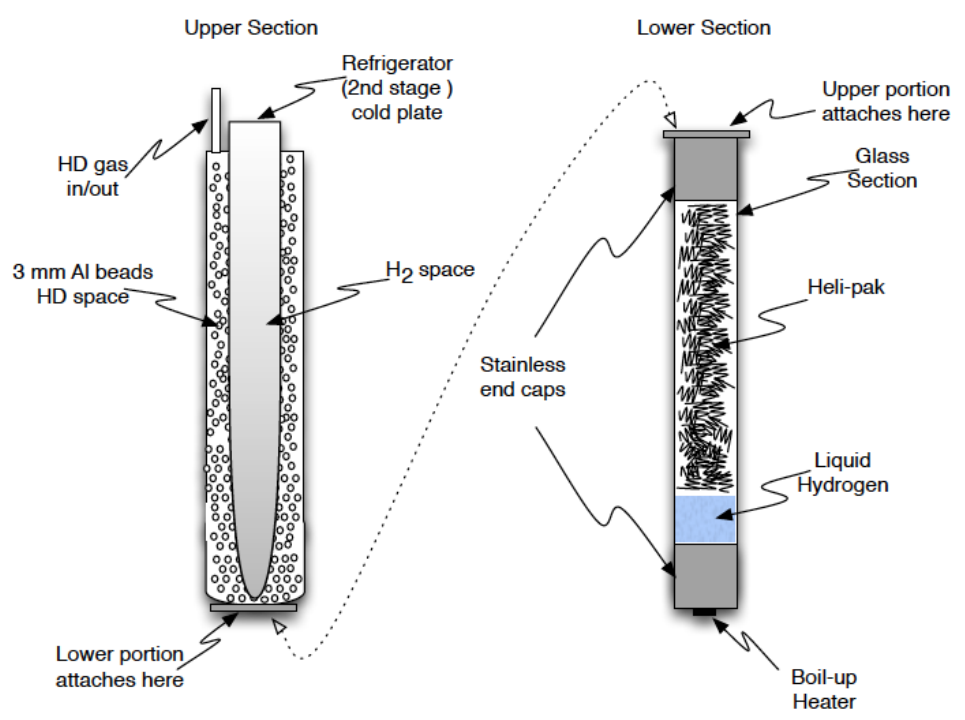

Figure 3.4: Schematic of the distillery. On the left the upper portion is shown; the packed column is shown on the right; the liquid hydrogen isomer mixture is boiled at the bottom of the lower portion of the column [49]. 


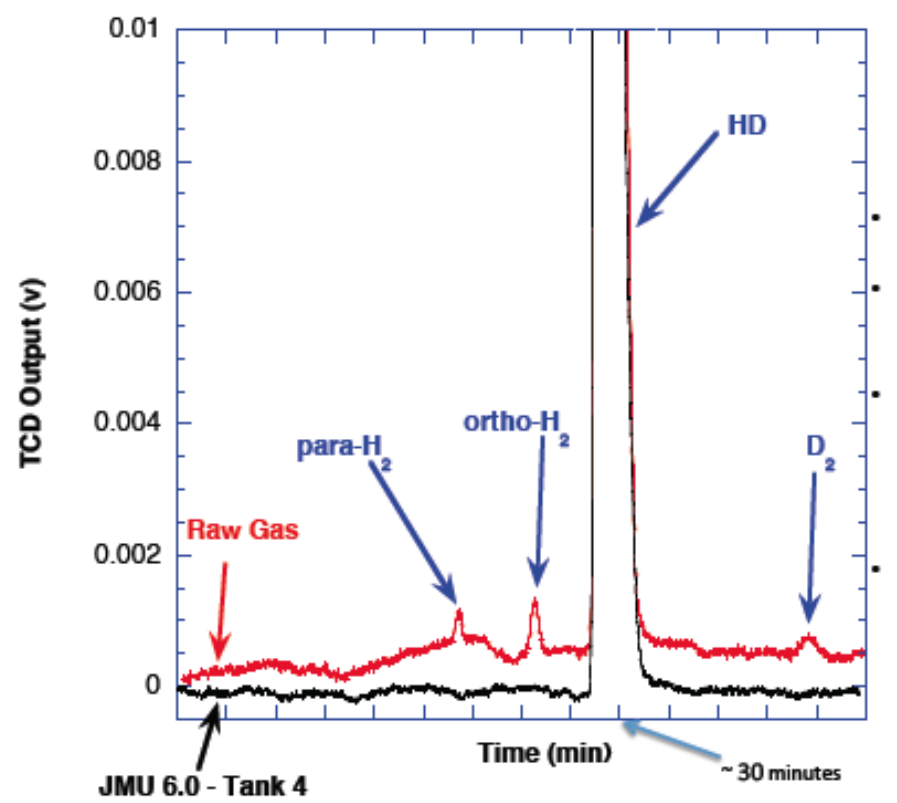

Figure 3.5: A comparison of the chromatogram of the raw, commercial gas and distilled gas. Note that in the distilled gas the $H_{2}$ and $D_{2}$ peaks are absent [49]. 


\subsection{HD Gas characterization}

To quantify the content of hydrogen isotopes with higher sensitivities, gas chromatography and Raman spectroscopy techniques may be employed.

The former technique consists in the measurement of the thermal conductivity difference of the gas to be analyzed with respect to a high purity Neon $(\mathrm{Ne})$ gas carrier, as a function of the retention time in a capillary column.

At JMU [50], a commercial instrument for gas chromatography was used, and sensitivities of the few parts $10^{-3}$ were reached for the hydrogen isotopes separation. This sensitivity, although sufficient to verify that the distillery is working correctly, is not high enough to measure contaminants concentrations at the level of $10^{-4}$, necessary for producing polarized "frozen spin" targets.

An example of a typical chromatogram is shown in Figure 3.5, where the comparison between the relative concentrations of $H_{2}$ and $D_{2}$ impurities in the raw gas and in the distilled gas is shown [49].

A valid approach to quantify with higher sensitivity the relative content of hydrogen isotopes is the Raman spectroscopy, which exploits the inelastic scattering of light from molecular hydrogen. For this purpose a Raman set-up was built at the University of Rome Tor Vergata, where our group carried out the measurements on a regular bases.

In the following sections the physics of the Raman scattering along with a brief description of the experimental setup used and the analysis method will be described.

\subsubsection{Rotational Raman spectroscopy}

When an electromagnetic radiation hits an atomic or molecular sample three scenarios are possible: if the energy of the incident radiation corresponds to the separation of two energy levels of the atoms or molecules the radiation may be absorbed, otherwise the radiation can be either transmitted or scattered by the sample. Most of the scattered radiation has an unchanged wavelength $\lambda$ and this corresponds to the elastic Rayleigh scattering. It can be proved that the intensity of the scattered light is related to $\lambda$ by the following relation:

$$
I_{s} \propto \lambda^{-4}
$$

In 1928 Raman and Krishnan showed experimentally that a small amount of radiation scattered by a gas, liquid or solid is of increased or decreased wavelength with respect to the original radiation. This is called the Raman effect and the scattered radiation having the decreased or the increased wavelength is referred to as Stokes or Anti-Stokes Raman scattering, respectively. 


\subsubsection{Theory of rotational Raman scattering}

An important property related to a macroscopic sample is the polarizability $\alpha$ from which the degree of scattering of an incident radiation depends. The polarizability may be intended as a measure of the degree to which the electrons in the molecule can be displaced relative to the nuclei; it is an anisotropic property, since at equal distances from the centre of the molecule it may have different magnitudes if measured in different directions. A pictorial description of the polarizability may be introduced, considering the surface each point of which is distant from a common origin $\alpha^{-\frac{1}{2}}$. This surface forms the polarizability ellipsoid, as shown in Figure 3.6. Like other anisotropic properties such as the moment of inertia of a molecule, polarizability is a tensor property and it may be expressed by the following matrix:

$$
\left(\begin{array}{lll}
\alpha_{x x} & \alpha_{x y} & \alpha_{x z} \\
\alpha_{y x} & \alpha_{y y} & \alpha_{y z} \\
\alpha_{z x} & \alpha_{z y} & \alpha_{z z}
\end{array}\right)
$$

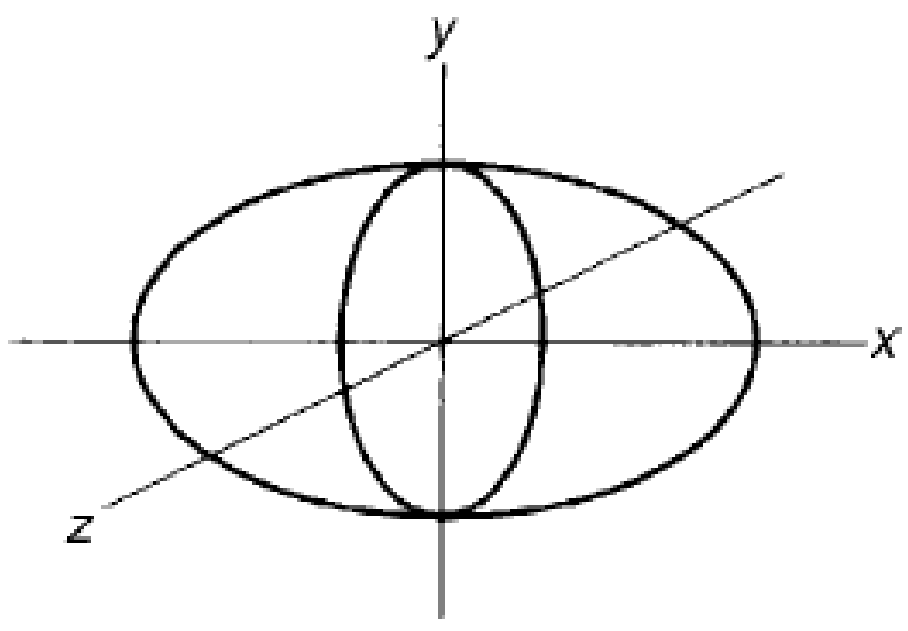

Figure 3.6: The polarizability ellipsoid [51].

where the diagonal elements are the values of $\alpha$ along the $\mathrm{x}, \mathrm{y}$ and $\mathrm{z}$ axes of the molecule, respectively. The matrix is symmetrical, so there are only 6 independent elements: $\alpha_{x x}, \alpha_{y y}, \alpha_{z z}, \alpha_{x y}, \alpha_{x z}$ and $\alpha_{y z}$.

When a monochromatic radiation hits a molecular sample in the gas phase without being absorbed, the oscillating electric field $\mathbf{E}$ of the radiation induces an electric dipole $\mu$ in the molecule, which is related to $\mathbf{E}$ by the following relation: 


$$
\boldsymbol{\mu}=\boldsymbol{\alpha E}
$$

where $\boldsymbol{\mu}$ and $\mathbf{E}$ are vector quantities. The magnitude $\mathrm{E}$ of the electric field as a function of time may be written:

$$
E=A \sin (2 \pi c \tilde{\nu} t)
$$

where $\mathrm{A}$ and $\tilde{\nu}$ are the amplitude and the wavenumber of the monochromatic incident radiation, respectively, and $\mathrm{c}$ is the light speed.

The magnitude of the polarizability will vary as the molecule rotates. The polarizability ellipsoid rotates with the molecule at a frequency $\nu_{\text {rot }}$ but the radiation sees the polarizability changing at twice the frequency of rotation since, as can be seen from Figure 3.6, the ellipsoid appears to be the same for a rotation of $\pi$ radians about any of the cartesian axes. The variation of the polarizability $\alpha$ with rotation is given by:

$$
\alpha=\alpha_{0, r}+\alpha_{1, r} \sin \left[2 \pi c\left(2 \nu_{\text {rot }} t\right)\right]
$$

where $\alpha_{0, r}$ is the average polarizability and $\alpha_{1, r}$ is the amplitude of the change of polarizability during rotation.

If we substitute equations 3.8 and 3.7 into equation 3.6 we get the magnitude of the induced dipole moment:

$$
\mu=\alpha_{0, r} A \sin (2 \pi c \tilde{\nu} t)-\frac{1}{2} \alpha_{1, r} A \cos \left[2 \pi c\left(\tilde{\nu}+2 n \tilde{u_{\text {rot }}} t\right]+\frac{1}{2} \alpha_{1, r} A \cos \left[2 \pi c\left(\tilde{\nu}-2 \tilde{\nu}_{\text {rot }} t\right]\right.\right.
$$

The terms in equation 3.9 represent scattering of the radiation. In particular, the first terms corresponds to Rayleigh scattering of unchanged wavenumber $\tilde{\nu}$, the second and third terms correpond to anti-Stokes and Stokes Raman scattering, with associated wave-numbers $\left(\tilde{\nu}+2 \tilde{\nu_{\text {rot }}}\right)$ and $\left(\tilde{\nu}-2 \tilde{\nu_{\text {rot }}}\right)$, respectively. It is of primary importance to note that, while in a classical system $\nu_{\text {rot }}$ may take any values, in a quantum mechanical system only certain values are possible, as it will be explained in the next section.

\section{Rotational Raman spectra of diatomic molecules}

In a diatomic (or linear polyatomic) molecule rotational Raman scattering obeys to the following selection rules:

$$
\Delta J= \pm 2
$$

( $\Delta \mathrm{J}=0$ transitions would correspond to the Rayleigh scattering). Besides, the molecule must have an anisotropic polarizability, so that $\alpha$ is not the same in all directions. This is not a very stringent requirements since all molecules except spherical 
rotors (whose polarizability is in the form of a sphere) have this property. As a result, all diatomic molecules, whether or not they have an inversion centre i, show a rotational Raman spectrum. The resulting Raman spectrum is shown in Figures 3.7 and 3.8 shows the process involved in the first Stokes and anti-Stokes transition and in the Rayleigh scattering.

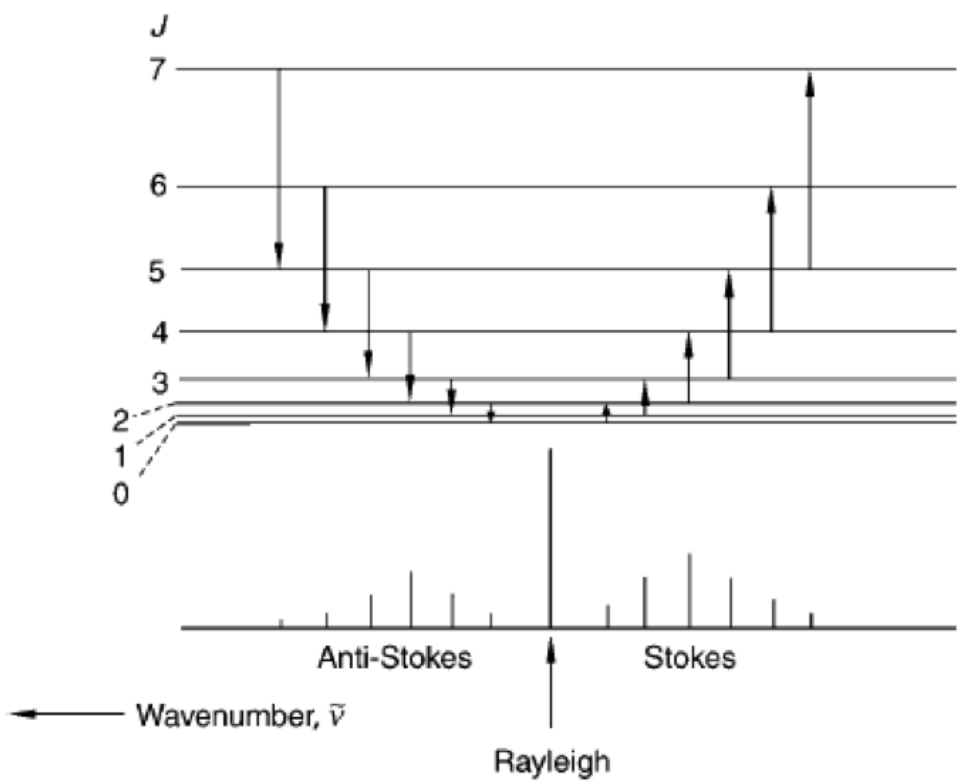

Figure 3.7: Rotational Raman spectrum of a diatomic or linear polyatomic molecule [51].

A molecule which is found initially in a $\mathrm{J}$ rotational state encounter an intense, monochromatic radiation, of wavenumber $\tilde{\nu}$. It may change its level, according to the section rules 3.10 while the scattered radiation is shifted in energy. The process is interpreted saying that the molecule is excited and, when scattering occurs, the molecule will decay to another rotational state under the condition $\Delta J=2$ and the radiation wave-length is changed by:

$$
\Delta \tilde{\nu}=\tilde{\nu}-\tilde{\nu}_{L}
$$

and

$$
|\Delta \tilde{\nu}|=F(J+2)-F(J)
$$

where $\mathrm{F}(\mathrm{J})$ is the molecular energy level change: 


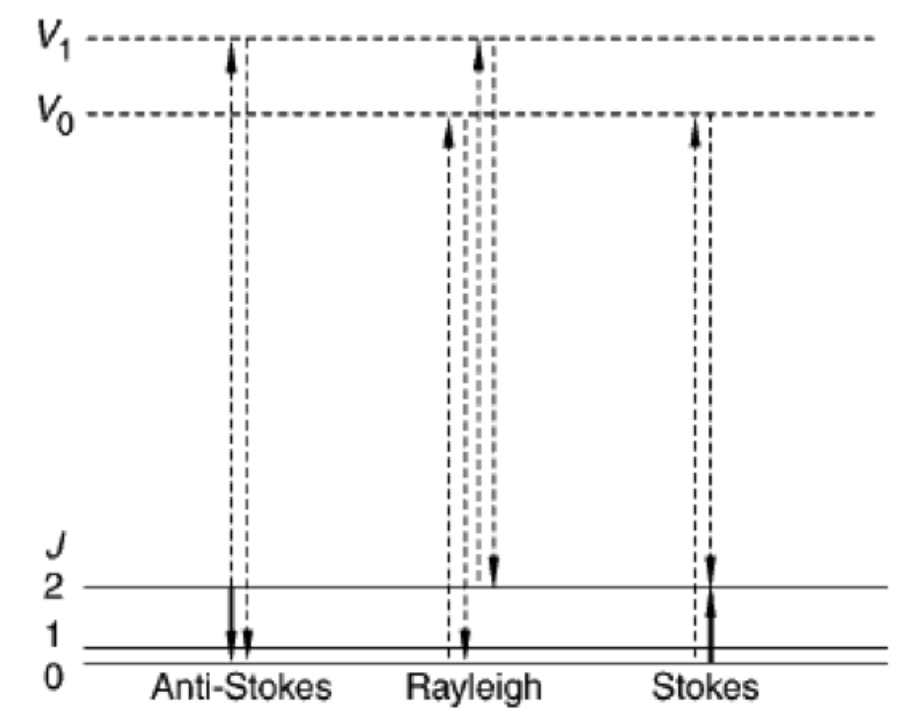

Figure 3.8: Raman and Reyleigh scattering processes involving virtual states V0 and V1 [51].

$$
F(J)=\frac{E_{r}}{h c}=\frac{h}{8 \pi^{2} c I} J(J+1)=h c B J(J+1)
$$

where $E_{r}$ are the rotational energy levels of a diatomic molecule, I is the moment of inertia and $\mathrm{B}$ is the rotational Raman constant:

$$
B=\frac{h}{8 \pi^{2} c I}
$$

$\tilde{\nu}_{L}$ is the wavenumber of the exciting laser radiation. $\Delta \tilde{\nu}$ is positive for antiStokes and negative for Stoke lines. At a first approximation, the energy shift of the Raman scattered radiation is given by:

$$
|\Delta \tilde{\nu}|=4 B_{0} J+6 B_{0}
$$

where centrifugal distortion in neglected. This relation holds for molecules in the zero-point vibrational states. The spectrum shows two sets of equally spaced lines with a spacing of $4 B_{0}$ and a separation of $12 B_{0}$ between the first Stokes and anti-Stoke lines.

Series of rotational transitions are called branches and they are labelled with a letter, according to the value of $\Delta J$ as shown in table 3.1: 


\begin{tabular}{|l|l|l|l|l|l|}
\hline$\Delta J$ & -2 & -1 & 0 & +1 & +2 \\
\hline Branch & $\mathrm{O}$ & $\mathrm{P}$ & $\mathrm{Q}$ & $\mathrm{R}$ & $\mathrm{S}$ \\
\hline
\end{tabular}

Table 3.1: Rotational transitions (branches) labelled according to $\Delta J$ values [51].

\subsubsection{Raman spectroscopy set-up and procedures}

The Raman measurements were performed in the Raman laboratory at the University of Rome Tor Vergata. The main components of the Raman apparatus consist of a high intensity Argon-Ion Sabre Inova Laser (15 W), a target cell specifically designed to perform Raman measurements on a gaseous sample and a spectrometer, made up by a monochromator and a CCD (Charged Coupled Device). A schematic view of the Raman apparatus is provided in Figure 3.9.

The light emission from the Laser is set to a wavelength of 514 nanometer, which is the highest intensity line available.

The light passes through an optical beam expander in order to produce a parallel beam. The beam is focused in the center of the target by a 1 inch focusing lens.

The geometry of the apparatus is designed in such a way that the beamline passing through the cell is directed at $90^{\circ}$ with respect to the entrance window of the monochromator. This minimized the amount of Laser light entering the spectrometer. Since the intensity of the Raman scattered light is very low, two concave mirrors (1 and 2 inches, respectively) are used to double the intensity of the incoming Laser light and scattered Raman light. The 1 inch mirror is placed along the beamline immediately after the target cell (see Figure 3.9) in order to focus back the laser light in the center of the target. The two inches mirror is placed at $90^{\circ}$ with respect to the beamline (see Figure 3.9), in order to collect the Raman light and reflects it towards the spectrometer. The Raman scattered light exits through the target cell window which faces the monochromator entrance and passes through a 2 inches convex-plano lens, which allows to the light to be propagated as a parallel beam.

Before reaching the monochromator entrance window, the beam passes through a second 2 inches plano-convex lens which focuses the light towards the first mirror inside the monochromator.

The light inside the monochromator passes through a diffraction lattice, where different wavelengths follow different optical paths, and is focused towards the CCD. The CCD, made up by a silicon pixel matrix, allows to acquire the spectrum as a function of the wavelength, because the monochromator focuses light of different wavelengths towards CCD pixels in different positions. In order to reduce thermal background noise the CCD is cooled using liquid Nitrogen (LN2). 


\section{The target cell and the filling procedure}

The target cell, specifically designed to perform Raman spectroscopy measurements of gaseous samples, is made of a cubic stainless steel core. A schematic drawing and pictures of the cell are shown in Figures 3.10 and 3.11.

The cubic core possesses four windows through which the laser and Raman scattered light passes through. The available volume for the gas inside the cubic core is 17 cc and it is internally shaped as follows. Each window is the basis of a cone whose vertex lies in the center of the cell: in this way the four cones (one for each window) intercept each other in the center of the cell. This particular shape allows to avoid accidental reflections inside the cell, which could interfere with the Raman light.

On the top of the cell, a stainless steel pipe is mounted, through which the HD gas can flow during the filling procedures. The pipe forms a "T-link" with another stainless steel pipe where a two-way stainless steel valves is placed and isolates the cell from the pipes. The cell can be easily removed from its support in the Raman apparatus to fill it with the HD gas sample. In the filling procedure, one output of the T-link is connected to a high vacuum pump and the other to the sample gas bottle. The high-vacuum pump, consists of a rotative pump, which can reach a vacuum up to $10^{-3}$ mbar, and a turbo-molecular pump, which can reach a vacuum up to $10^{-7}$ mbar.

At each filling the cell is pumped to vacuum up to the HD bottle valve. Then the pump valve is closed and the gas valve is opened for few seconds to allow gas expanding from the bottle into the cell. The HD gas bottle valve and the cell valve are closed to isolate the cell and disconnect it from the filling manifold.

It is of primary importance to fill the cell just before performing the Raman measurements, since it has been experimentally observed that the HD gas recombine in $\mathrm{H}_{2}$ and $\mathrm{D}_{2}$ more frequently in the target cell than in the storage bottle. 


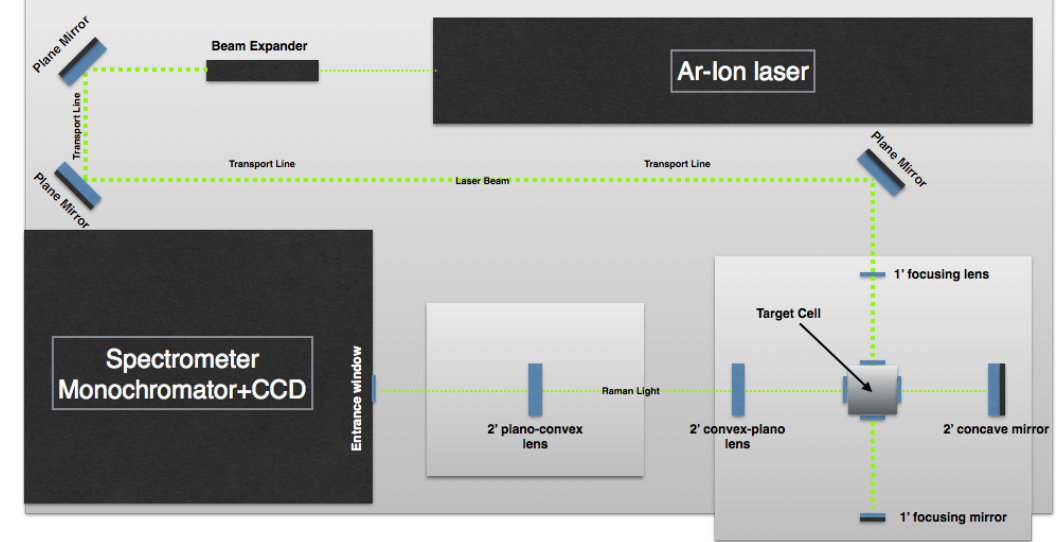

Figure 3.9: Right: schematic of the Raman experimental apparatus.
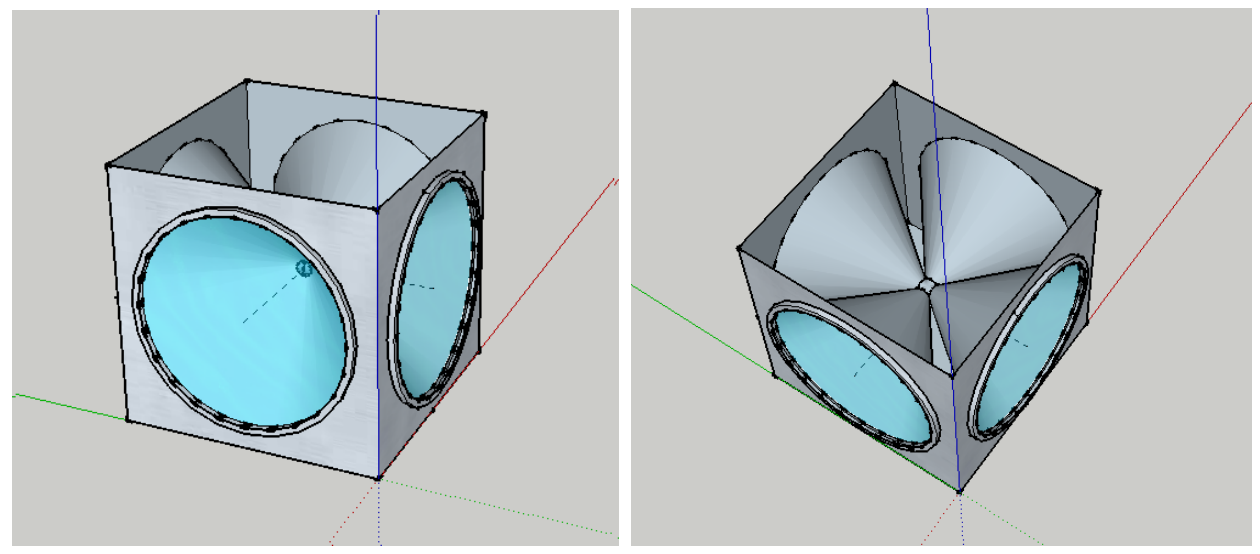

Figure 3.10: Sketch of the Raman Target cell. 

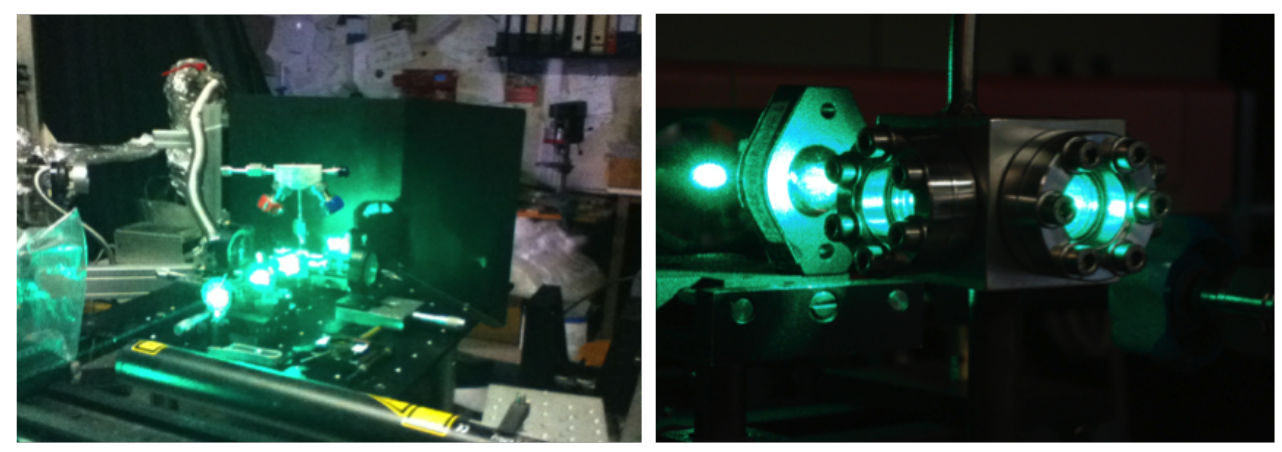

Figure 3.11: Raman Target cell filled with HD gas hit by the high intensity green light of the laser to perform Raman spectroscopy measurement.

\subsection{Raman analysis results}

The Raman data analysis consists of the experimental determination of the Raman peaks intensities, as a function of the rotational eigen-value $\mathrm{J}$ of each species and the gas temperature, which are described by the following relation:

$$
I(J, T)=I_{0} A(\nu) \nu^{3} f(J) \gamma^{2} \frac{45 \pi^{4}}{7} \frac{N}{Q(T)} g_{s}(J)(2 J+1) \frac{3(J+1)(J+2)}{2(2 J+1)(2 J+3)} e^{-\frac{h c b_{0} J(J+1)}{K T}}
$$

where $I_{0}$ is the Laser intensity, $A(\nu)$ is the spectral response of the spectrometer as a function of the light frequency $\nu, \mathrm{f}(\mathrm{J})$ is the anharmonicity correction of the rotational energy level $\mathrm{J}, \gamma^{2}$ is the anisotropic matrix element. The product:

$$
C=I_{0} A(\nu) \nu^{3} f(J) \gamma^{2} \frac{45 \pi^{4}}{7}
$$

is approximately constant for all lines and species, for a specific spectrum. $\mathrm{N}$ is the number of molecules of the species we want to determine, $\mathrm{Q}(\mathrm{T})$ is the partition function at temperature $\mathrm{T}$ and it is given by the following relation:

$$
Q(T)=\sum_{J} g_{s}(J)(2 J+1) e^{-\frac{h c b_{0} J(J+1)}{K T}}
$$

$g_{s}(J)$ is the nuclear spin multiplicity $2 \mathrm{I}+1$; its dependency on the rotational level and on the molecular species is illustrated in table 3.2. The quantity $E_{R}(J)=$ $h c b_{0} J(J+1)$ is the rotational energy level, being $b_{0}=\frac{K}{8 \pi^{2} I}$ the Raman constant, that we have already encountered in section 3.4.2 (formula 3.13). 


\begin{tabular}{|l|l|l|l|}
\hline Gas & $\left(2 I_{H}+1\right) \times\left(2 \mathrm{I}_{D}+1\right)$ & $\mathrm{g}_{s}(J)$ & \\
\hline HD gas & & $I_{H}=1 / 2 I_{D}=1$ & $g_{s}(J)=6$ \\
\hline Ortho- $H_{2}$ & J odd & $I_{H_{2}}=1$ & $g_{s}(J)=3$ \\
\hline Para- $H_{2}$ & J even & $I_{H_{2}}=0$ & $g_{s}(J)=1$ \\
\hline Para- $D_{2}$ & J odd & $I_{D_{2}}=1$ & $g_{s}(J)=3$ \\
\hline Ortho- $D_{2}$ & J even & $I_{D_{2}}=0,2$ & $g_{s}(J)=6$ \\
\hline
\end{tabular}

Table 3.2: Dependency of the nuclear spin multiplicity, $g_{s}(J)$ on the rotation level and molecular species.

In Figure 3.12 the measured Raman spectrum in logarithmic scale is shown. The peaks of the HD, $H_{2}$ and $D_{2}$ are clearly visible, and we can see that they correspond to transitions between ortho-ortho and para-para states, as allowed by the Raman scattering selection rules $\Delta J= \pm 2$ (see section 3.10).

Raman spectra are in general very clean and peaks are well described by a Gaussian fit on a constant background. So every single Raman peak is fitted using the function:

$$
f(x)=A e^{\frac{-\left(x-x_{0}\right)^{2}}{s^{2}}}+B
$$

where $x_{0}$ gives the peak position, the width is given by the parameter $\mathrm{s}$ and the amplitude over the background $\mathrm{B}$ is given by $\mathrm{A}$. The peak intensity is obtained by the relation:

$$
I(J)=\sqrt{\pi} A s
$$

Figure 3.14 shows typical fits of the five HD Raman lines.

One can rearrange the terms in formula 3.16 and using the semi-logarithmic scale we get:

$$
\ln \frac{I_{\text {meas }}(J)}{h(J)}=\ln \frac{C N}{Q(T)}-\frac{h c b_{0} J(J+1)}{K} \frac{1}{T}
$$

and we can fit the ratio:

$$
\ln \frac{I_{\text {meas }}(J)}{h(J)}
$$

as a function of the term:

$$
\frac{h c b_{0} J(J+1)}{K}
$$

from this we get a straight line from whose slope we get the Temperature and the intercept gives $\mathrm{CN} / \mathrm{Q}(\mathrm{T})$, as shown in Figure 3.14. 


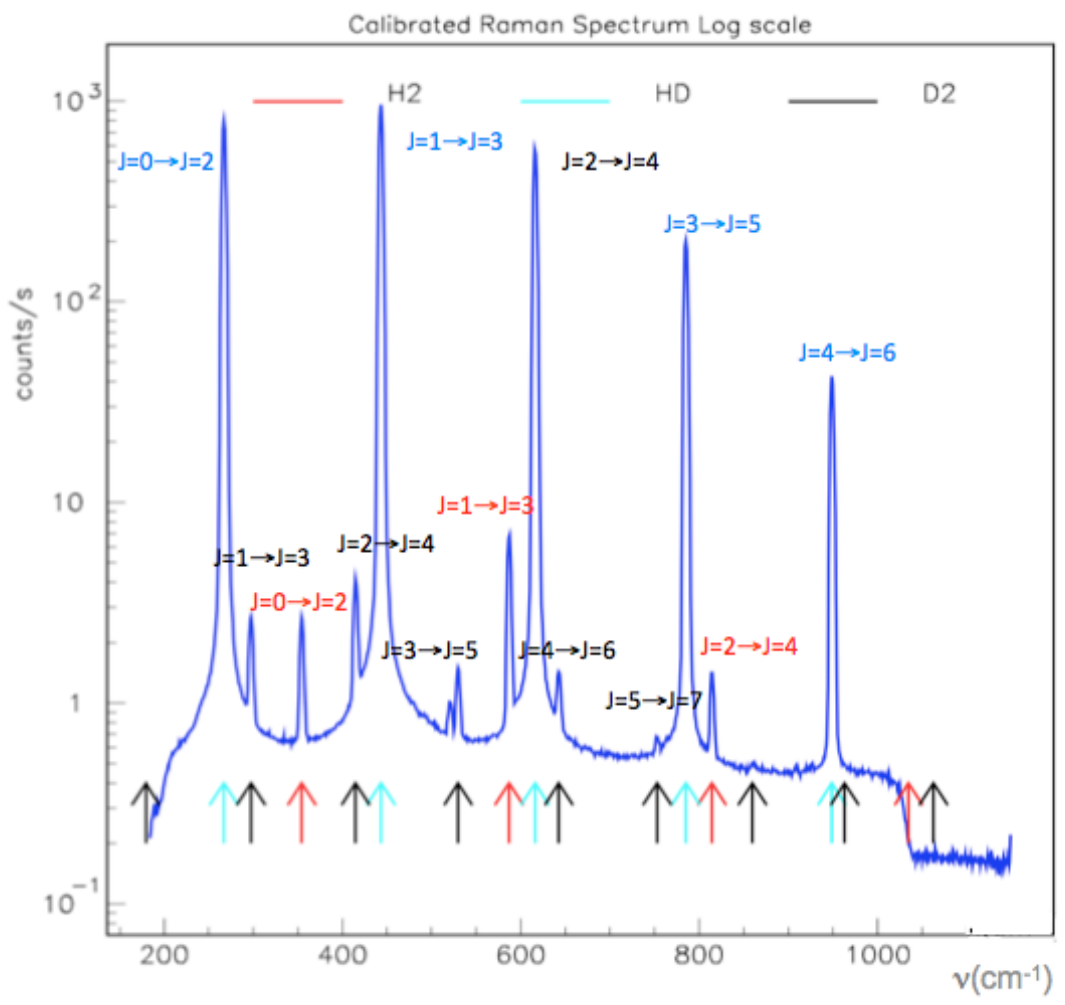

Figure 3.12: An example of calibrated Raman spectrum for HD JMU-III sample. Light blue arrows point to HD lines. Red arrows point to $H_{2}$ lines. Black arrows point to $D_{2}$ lines. The first five HD lines corresponding to transition among pure rotational states are visible. The first $\mathrm{H}_{2}$ lines corresponding to transition among para $(\mathrm{J}=0$ to $\mathrm{J}-2)$ ortho $(\mathrm{j}=1$ to $\mathrm{J}=3)$ and para- $(\mathrm{j}=2$ to $\mathrm{J}=4)$ states are visible. Five $D_{2}$ lines are visible starting from the first transition among para-states ( $\mathrm{J}=1$ to $\mathrm{J}-3$ ). The first $D_{2}$ line among ortho-states $(\mathrm{J}=0$ to $\mathrm{J}=2)$ is not covered by the recorded spectrum. 

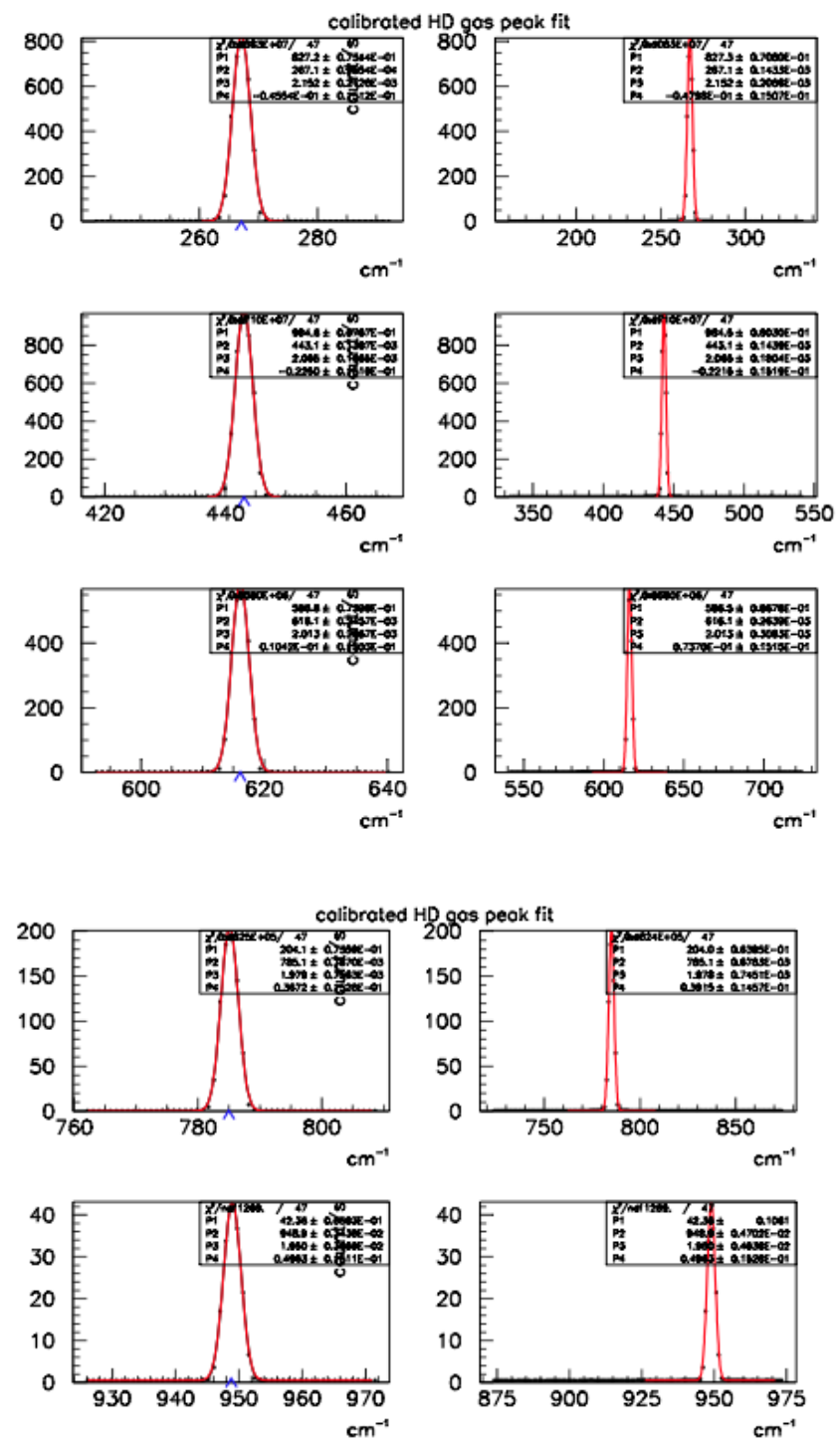

Figure 3.13: Gaussian plus constant background fit of the HD Raman peaks, shown in logarithmic vertical scale. 


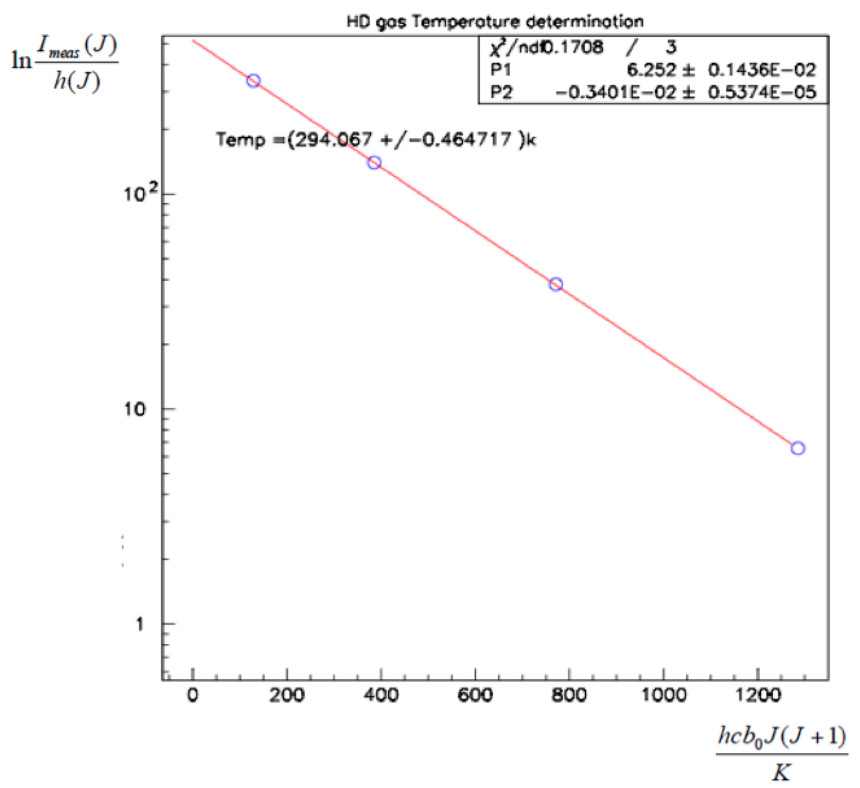

Figure 3.14: Linear fit of the ratio $\mathrm{I}(\mathrm{J}) / \mathrm{h}(\mathrm{J})$ in logarithmic scale as a function of $\mathrm{E}(\mathrm{J}) / \mathrm{K}$ for three $\mathrm{HD}$ peaks. The slope provides $1 / \mathrm{T}$ and the intercept provides $\ln (\mathrm{CN} / \mathrm{Q})$. 
Once the temperature value is extracted, it is possible to calculate the partition function $\mathrm{Q}(\mathrm{T})$ and determine for each peak the product:

$$
C N=\frac{I_{\text {meas }}(J)}{h(J)} Q(T) e^{-\frac{\left(h c b_{0} J(J+1)\right)}{K T}}
$$

which, at thermal equilibrium, should be independent from $\mathrm{J}$; a fit (weighted average) of the results provides the average value of the product for the single gas species. The same procedure is applied for each gas. it is also possible to separately analyze the content of ortho- and para- species separately; they correspond to the J-odd $\rightarrow$ J-odd and J-even $\rightarrow$ J-even Raman lines, respectively for $H_{2}$ and J-even $\rightarrow$ J-even and J-odd $\rightarrow$ J-odd Raman lines, respectively for $D_{2}$. The procedure is very similar, only the value of the partition function $\mathrm{Q}(\mathrm{T})$ must be appropriately calculated to include only the energy levels corresponding to J-odd (or J-even) rotational states.

In that way we get the values of $C N_{\text {tot }}, C N_{\text {para }}$ and $C N_{\text {orto }}$, as shown in Figure 3.15 , where $N_{\text {tot }}, N_{\text {para }}$ and $N_{\text {orto }}$ are the numbers of molecules of ortho, para and total respectively. $\mathrm{C}$, as said before, has the same values for the different species. At this point the relative content of the isotope can be evaluated calculating the ratios:

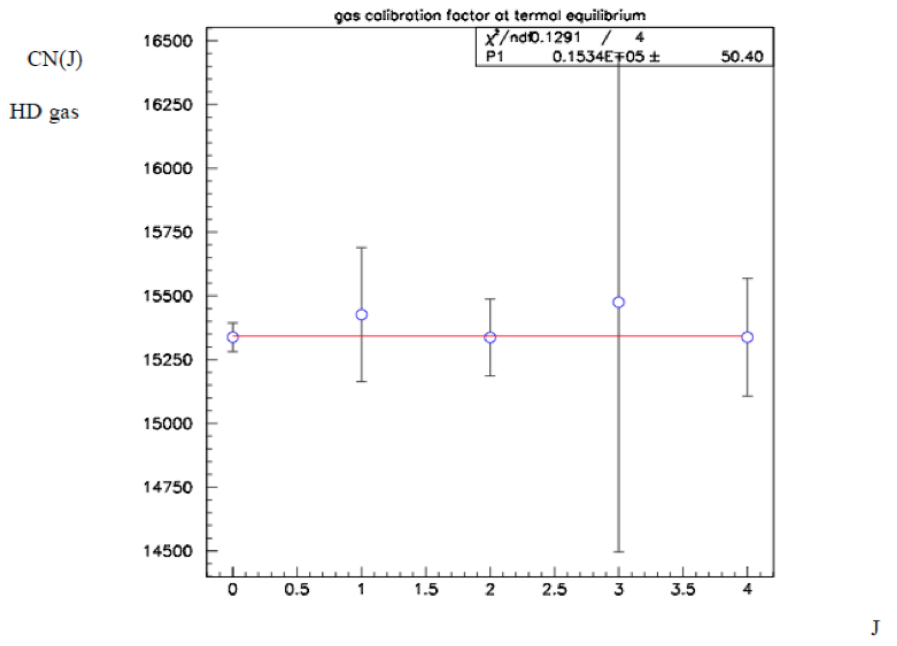

Figure 3.15: Weighted average of the five values of the product CN extracted from each of the five HD Raman peaks

$$
c_{\text {orto }}=\frac{C N_{\text {orto }}}{C N_{\text {tot }}}=\frac{N_{\text {orto }}}{N_{\text {tot }}} \quad c_{\text {para }}=\frac{C N_{\text {para }}}{C N_{\text {tot }}}=\frac{N_{\text {para }}}{N_{\text {tot }}}
$$

Using the information from the literature [52] on relative peak intensities of an isotopic equilibrated mixture of $H_{2}-H D-D_{2}$, we can extract the ratio of the 
constant $\mathrm{C}$ for different gasses, at room temperature. The intensity formula for each peak reduces to:

$$
I(J, T)=C N f(J, T)
$$

where

$$
f(J, T)=\frac{h(J)}{Q(T)} e^{\frac{-E_{R}(J)}{K T}}
$$

may be calculated for each peak at a fixed temperature, for each gas type. Imposing $N_{H D}=N_{H 2}[52]$ one obtains:

$$
\frac{I_{H D}(J=1)}{I_{H_{2}(J=1)}}=0.58=\frac{C_{H D} N_{H D} f_{H D}(J=1, T)}{C_{H_{2}} N_{H_{2}} f_{H_{2}}(J=1, T)}=\frac{C_{H D} f_{H D}(J=1, T)}{C_{H_{2}} f_{H_{2}}(J=1, T)}
$$

from this relation it is possible to extract the correction factor, among the different gases:

$$
\frac{C_{H D}}{C_{H_{2}}}=\frac{I_{H D}}{I_{H_{2}}} \frac{f_{H_{2}}(J=1, T)}{f_{H D}(J=1, T)}=0.58 \cdot \frac{f_{H D}(J=1, T)}{f_{H_{2}}(J=1, T)}=0.98756
$$

In a similar way:

$$
\begin{array}{r}
\frac{I_{D_{2}}(J=1)}{I_{H D}(J=1)}=\frac{I_{D_{2}}(J=1)}{I_{H 2}(J=1)} \cdot \frac{I_{H_{2}}(J=1)}{I_{H D}(J=1)}=\frac{0.47}{0.58}=0.8103 \\
=\frac{C_{D_{2}} N_{D_{2}} f_{D_{2}}(J=2, T)}{C_{H_{2}} N_{H_{2}} f_{H_{2}}(J=1, T)} \cdot \frac{C_{H_{2}} N_{H_{2}} f_{H_{2}}(J=1, T)}{C_{H D} N_{H D} f_{H D}(J=1, T)}=\frac{C_{D_{2}} f_{D_{2}}(J=2, T)}{C_{H D} f_{H D}(J=1, T)}
\end{array}
$$

from this relation one may extract the correction factor for $D_{2} / H D$ :

$$
\frac{C_{D_{2}}}{C_{H D}}=\frac{I_{D_{2}}(J=1)}{I_{H_{2}}(J=1)} \frac{I_{H_{2}}(J=1)}{I_{H D}(J=1)} \cdot \frac{f_{H D}(J=1, T)}{f_{D_{2}}(J=2, T)}=0.8103 \cdot \frac{f_{H D}(J=1, T)}{f_{D_{2}}(J=2, T)}=1.0343
$$

Finally it is possible to determine the $N\left(H_{2}\right) / N(H D)$ and $N(D 2) / N(H D)$ ratios as follows:

$$
\frac{N\left(H_{2}\right)}{N(H D)}=\frac{C N_{H 2}(f i t)}{C N_{H D}(f i t)} \cdot \frac{C_{H D}}{C_{H_{2}}}=0.98756 \cdot \frac{C N_{H_{2}}(f i t)}{C N_{H D}(f i t)}
$$

and

$$
\frac{N\left(D_{2}\right)}{N(H D)}=\frac{C N_{D 2}(f i t)}{C N_{H D}(f i t)} \cdot \frac{C_{H D}}{C_{D_{2}}}=1.0343 \cdot \frac{C N_{D_{2}}(f i t)}{C N_{H D}(f i t)}
$$




\begin{tabular}{|l|l|l|}
\hline$H 2 / H D$ & JMU-II & USC \\
\hline Raman & $0.00472 \pm 0.00004$ & $0.00387 \pm 0.00004$ \\
\hline GC & $0.0049 \pm 0.0002$ & $0.0034 \pm 0.0007$ \\
\hline
\end{tabular}

Table 3.3: Relative concentration $H_{2} / H D$ determined through Raman spectroscopy and Gas Chromatography (GC) measurements.

\begin{tabular}{|l|l|l|}
\hline$D 2 / H D$ & JMU-II & USC \\
\hline Raman & $0.00416 \pm 0.00008$ & $0.00442 \pm 0.00008$ \\
\hline GC & $0.0014 \pm 0.0002$ & $0.0033 \pm 0.0032$ \\
\hline
\end{tabular}

Table 3.4: Relative concentration $D_{2} / H D$ determined through Raman spectroscopy and Gas Chromatography (GC) measurements.

The described procedure was applied to determine the relative concentration of ortho $-\mathrm{H}_{2}$ and para $-\mathrm{D}_{2}$ in the HD for the two gases USC e JMUII which have been used for the silver 5 and gold 2 target, respectively (see table 4.1 of section). The results of the measurements for these two gases are shown in table 3.3 and 3.4. The results obtained with Raman Spectroscopy technique are compared with those obtained with Gas Chromatography measurements (GC) (see section 3.4); it can be seen that these latter are not sensitive enough to measure the optimal concentration of ortho $-H_{2}$ and para $-H_{2}$ impurities required to polarize the HD crystal.

\subsection{The HD-ice Target equipment: cryostats}

If, from one side, the polarized target frozen-spin HD has many attractive features, from the other side its production can be considered a very hard task, considerably more complex than that of conventional polarized nuclear targets.

One aspect of this complexity involves the need of several specifically designed and constructed cryostats and target manipulation tools, as well as a number of commercial dewars, magnetic systems and dilution refrigerator. The cryogenic devices needed for the HDice target were:

- The Production Dewar (PD): used for target condensation and polarimetry measurements with a temperature of $2 \mathrm{~K}$ and a magnetic field up to $3 \mathrm{~T}$. 
- The Transfer Cryostat: used to extract the solid HD target from the PD and transfer it to the other cryostats with a temperature of $2 \mathrm{~K}$ and a magnetic field of $0.1 \mathrm{~T}$.

- The Dilution Fridge: used to polarized the target with a temperature of 10 $\mathrm{mK}$ and a magnetic field of $15 \mathrm{~T}$.

- The Storage Dewar: used for the storage and the transportation of the target with a temperature of $1.7 \mathrm{~K}$ and a magnetic field of $6 \mathrm{~T}$

- The In-Beam Cryostat: used to operate the target under a particle beam with a temperature of $50 \mathrm{mK}$ to $300 \mathrm{mK}$, a longitudinal magnetic field up to $0.9 \mathrm{~T}$ and a transverse magnetic field up to $0.07 \mathrm{~T}$.

They will be discussed in the next sections.

\subsubsection{The Production Dewar}

The Production Dewar (PD) cryostat was used for the production of the target cell, to perform NMR measurement as well as cell characterization. The cell was mounted on a copper pedestal in the cold finger of the $\mathrm{PD}$, which also contained a pair of radio frequency could used for polarimetry.

The cooling of the cell was provided by liquid helium contained in a space called "VariTemp" (see Figure 3.16), fed by the helium bath through a needle valve. The operation temperature of the cell in the PD was about $4 \mathrm{~K}$, but it could be lowered below $2 \mathrm{~K}$ by pumping on the surface of the helium in the VariTemp space. Since the needle valve was manual, the filling was done once a day from the target group both for liquid nitrogen and liquid helium. The magnetic field, up to $3 \mathrm{~T}$, was provided by a superconducting magnet.

\subsubsection{The Transfer Cryostat}

The Transfer Cryostat (TC) [46], was needed for transferring the cell from a cryostat to another one and for transporting the target from the HDice lab, where the targets were produced, to the experimental hall, where the targets were used for conducting the g14 experiment.

The Transfer Cryostat consisted of a liquid helium tube $3 \mathrm{~m}$ long, that can be pumped and cooled down to $2 \mathrm{~K}$.

The tube allowed to access the cell in the other cryostats through the left-handed threaded "coldhead" on its end. The coldhead was a threaded piece of copper that was screwed into the inner thread of the target cell ring.

Pre- cooling was provided by a double-walled liquid nitrogen filled tube. A radiation shutter was also available to reduce the liquid helium consumption. 


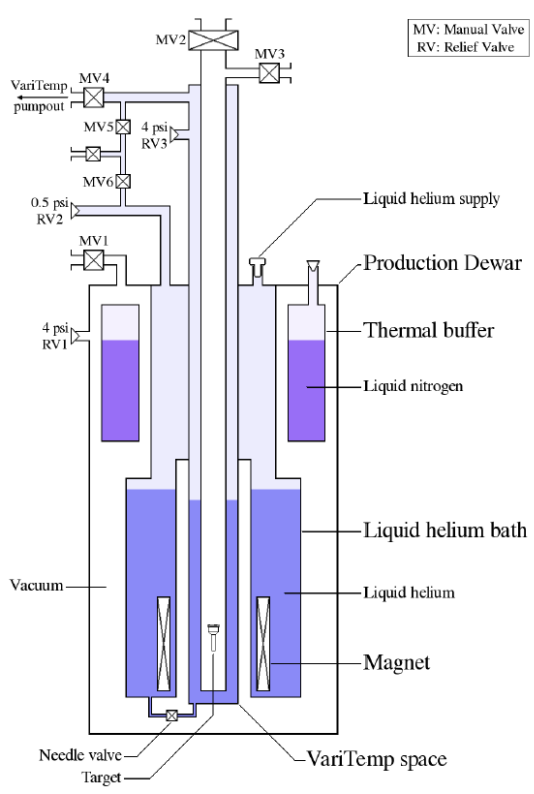

Figure 3.16: The Production Dewar used for the HD-ice target [47].

A picture and a sketch of the transfer cryostat are provided in Figure 3.17 and 3.18, respectively. When the cell was mounted in the TC, the holding magnetic field, necessary to preserve the target polarization, was provided by a Hallbach cylinder which produced a permanent transverse magnetic field of $0.1 \mathrm{~T}$. The transverse direction of the magnetic field allowed for the extraction and the insertion of the cell regardless the orientation of the longitudinal magnetic field of the cryostat. Indeed, if the TC had instead a longitudinal magnetic field, the orientation of all magnetic fields should match or a zero field region would exist on the path of the cell transfer. Because of the relatively low magnetic field and the relatively high temperature, the polarized cell could not remain in the TC for too long as the relaxation time decreases considerably in these condition. Typical transfer period could vary from 40-60 minutes, during which the HD target showed very little polarization loss, $\mathrm{H}$ $(-1 \% \pm 2$, relative $)$ or $\mathrm{D}(0 \% \pm 3$, relative $)[46]$.

\subsubsection{The Dilution Fridge}

The Dilution Fridge (DF) was used to polarize the HD-ice target; its design permitted to polarized up to 3 target cells simultaneously. A superconducting magnet provided a magnetic field of $15 \mathrm{~T}$ when it was cooled down at $4.2 \mathrm{~K}$ and up to $17 \mathrm{~T}$ at 2.2 $\mathrm{K}$. The cooling of the DF down to $10 \mathrm{mK}$, was achieved with a mixture of ${ }^{3} \mathrm{He}$ and ${ }^{4} \mathrm{He}$, which undergoes spontaneous phase separation when cooled below a critical 


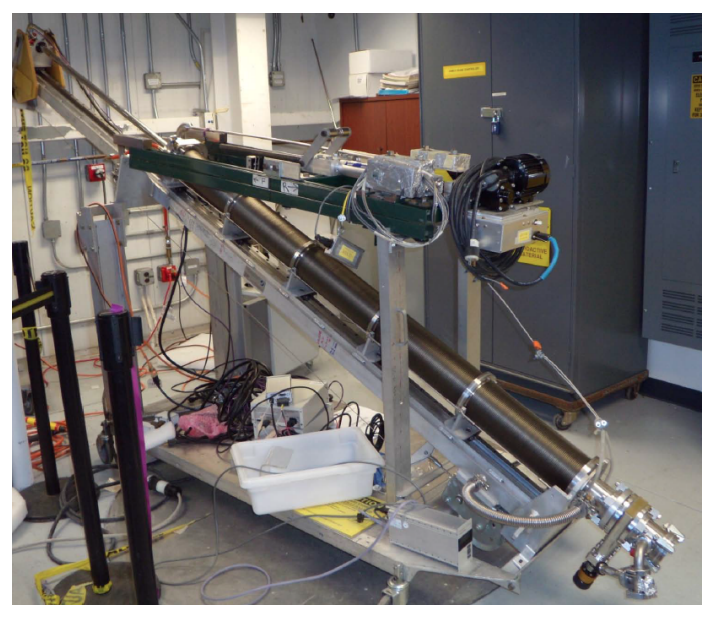

Figure 3.17: The Transfer Cryostat used for the HD-ice target.

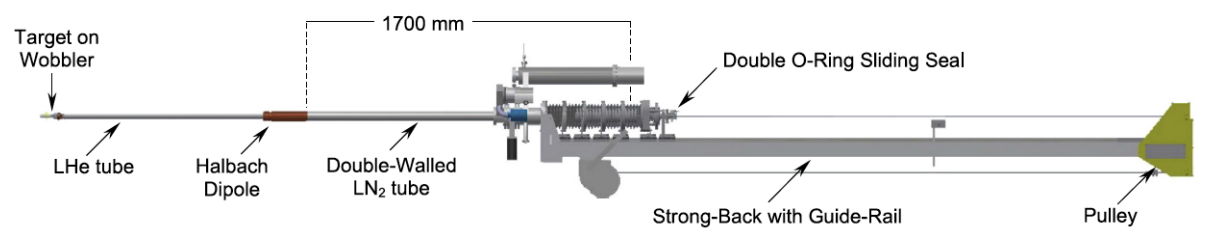

Figure 3.18: Schematic of the Transfer Cryostat in its extended configuration used to reach the cold-bore of another cryostat when coupled thorough a vacuum gate valve [46]. 
temperature $(0.86 \mathrm{mK})$ to form a lighter concentrated phase rich in ${ }^{3} \mathrm{He}$ (almost $\left.100 \%{ }^{3} \mathrm{He}\right)$ and a dilute phase rich in ${ }^{4} \mathrm{He}\left(\sim 6.6 \%{ }^{3} \mathrm{He}\right.$ and $\left.93.4 \%{ }^{4} \mathrm{He}\right)$. The concentration of ${ }^{3} \mathrm{He}$ in each phase is temperature-dependent. Since the enthalpy of the ${ }^{3} \mathrm{He}$ in the two phases is different, the evaporation of ${ }^{3} \mathrm{He}$ from the concentrated phase (where the ${ }^{3} \mathrm{He}$ is liquid) into the dilute one (where the ${ }^{3} \mathrm{He}$ is a gas) provides effective cooling power.

The initial ${ }^{3} \mathrm{He} /{ }^{4} \mathrm{He}$ gas mixture was stored in a gas tank. It was first purified by a liquid nitrogen trap, pre-cooled by the main helium bath and then condensed by the 1 Kpot in the dilution unit.

The $1 \mathrm{~K}$ pot was a pumped helium volume, similar to the VariTemp space of the Production Dewar. The flow of liquid helium was adjusted by a needle valve to maintain temperature of $1.3 \mathrm{~K}$. The final cooling of the mixture (down to the critical temperature) was operated by the still before entering the heat exchangers and the mixing chambers. Gradually the pure ${ }^{3} \mathrm{He}$ dissolves into the diluted phase, the molecules cool down as they expand freely into the superfluid ${ }^{4} H e$. This allowed to cool down the mixing chambers and the target cells since they were thermally connected with copper parts. The ${ }^{3} \mathrm{He}$ was distilled out at the still and eventually moved back to the condensing side to rejoin the process. On its way out, to increase the efficiency of the system, the cold mixture cools down the incoming mixture going through the heat exchanger and the still. A schematic and a picture of the Dilution Fridge is provided in Figures 3.19.

\subsubsection{The Storage Dewar}

The Storage Dewar (SD) was used for the storage of three target cells simultaneously which were in a frozen spin state, freeing the DF unit and allowing to start the production of other 3 cells. Its structure was pretty similar to the one of the Production Dewar (see section 3.6.1). In Figure 3.20 a picture of the SD is shown. The SD could be cooled down to $1.6 \mathrm{~K}$ by pumping a VariTemp space, in a process remotely controlled by a computer. The main features of the SD were its outstanding cryogenic capacity since it needed to be filled every two days and its very strong holding magnetic field of $\sim 6 T$. The SD could also be used to transport the polarized targets among different laboratories.

\subsubsection{The In-Beam Cryostat}

The In-Beam Cryostat (IBC) was a dilution refrigerator purpose-built to operate under a particle beam. For this scope, the IBC was built with the minimum amount of material around the cell to reduce the energy loss of the particles coming in and out of it; for example, the walls were made of a layer of aluminum $1 \mathrm{~mm}$ thick and the two superconducting magnets have a limited number of layers of superconducting wires. The IBC structure permitted to rotate it between vertical and horizontal orientations, 

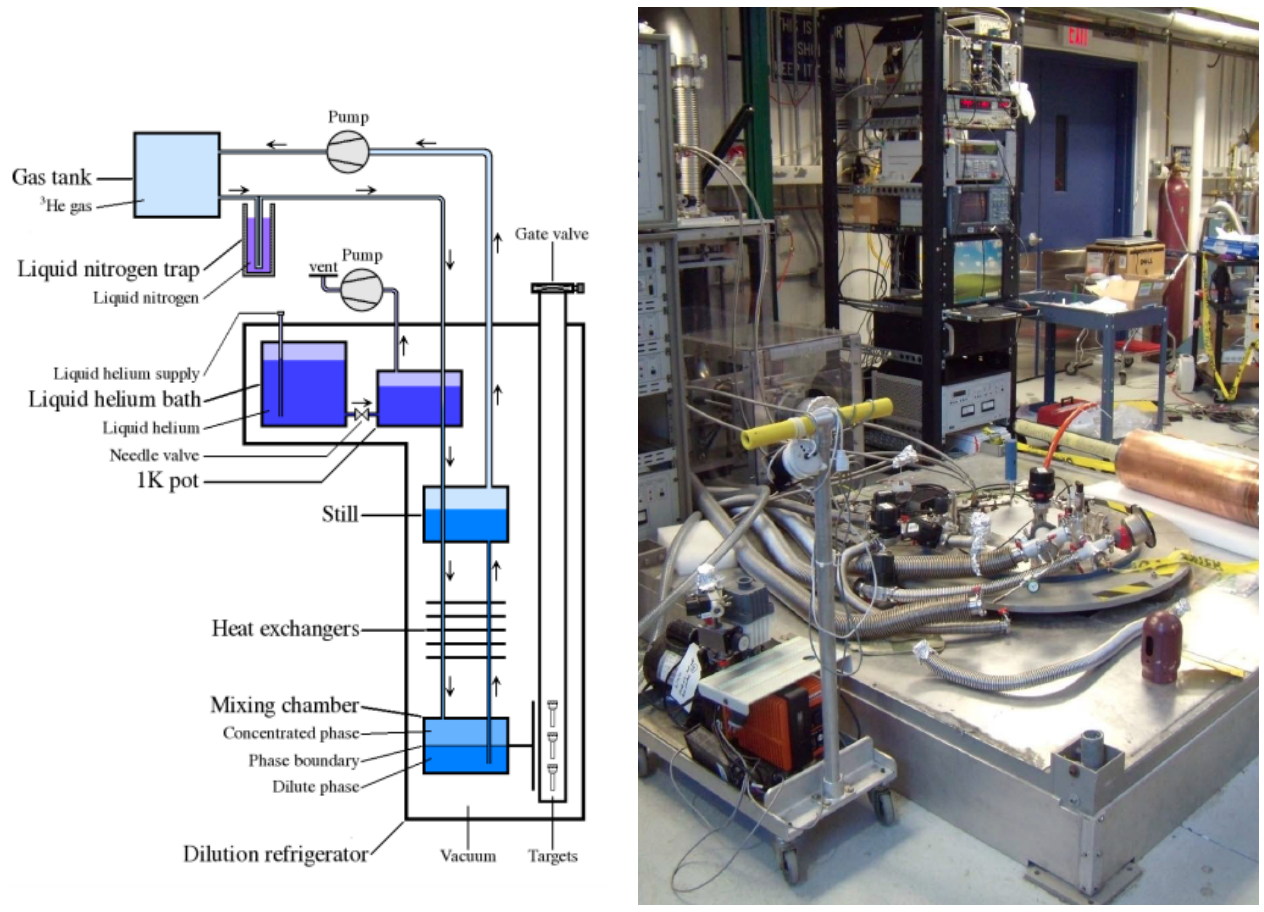

Figure 3.19: Left: Schematic of the dilution refrigerator [47]. Right: Picture of the dilution refrigerator in the HDice laboratory. 


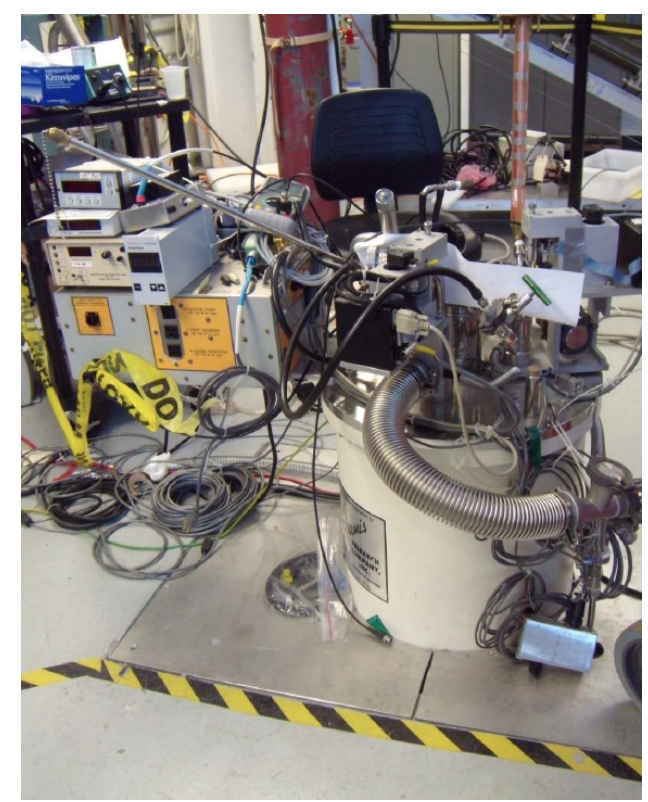

Figure 3.20: The Storage Dewar used for the HD-ice target

allowing for the transfer of the target cell into the IBC while was vertical, and for the alignment of the target cell with the beam to conduct the experiment while was horizontal (see Fig. 3.21). The cooling system of the IBC was similar to the one of the $\mathrm{DF}$ and could reach temperatures down to $50 \mathrm{mK}$.

The IBC was remotely controlled by computer and the cryogenic filling could be done automatically. The IBC was equipped with two superconducting magnets, a solenoid that provided a longitudinal magnetic field of $0.9 \mathrm{~T}$ and a so-called "saddle coil" magnet that provided a transverse magnetic field of $0.07 \mathrm{~T}$. This latter also allowed the rotation of the longitudinal magnet field which permitted to flip the polarity without creating a zero field period during the process, which could cause the loss of polarization of the cell. To preserve the polarization in case of a magnet quench a non-superconducting coil was necessary. For this purpose a "back-up" coil of $0.01 \mathrm{~T}$ cooled down with water, was wound on the outside of the cryostat. A sketch and a picture of the IBC is shown in Figures 3.21. 

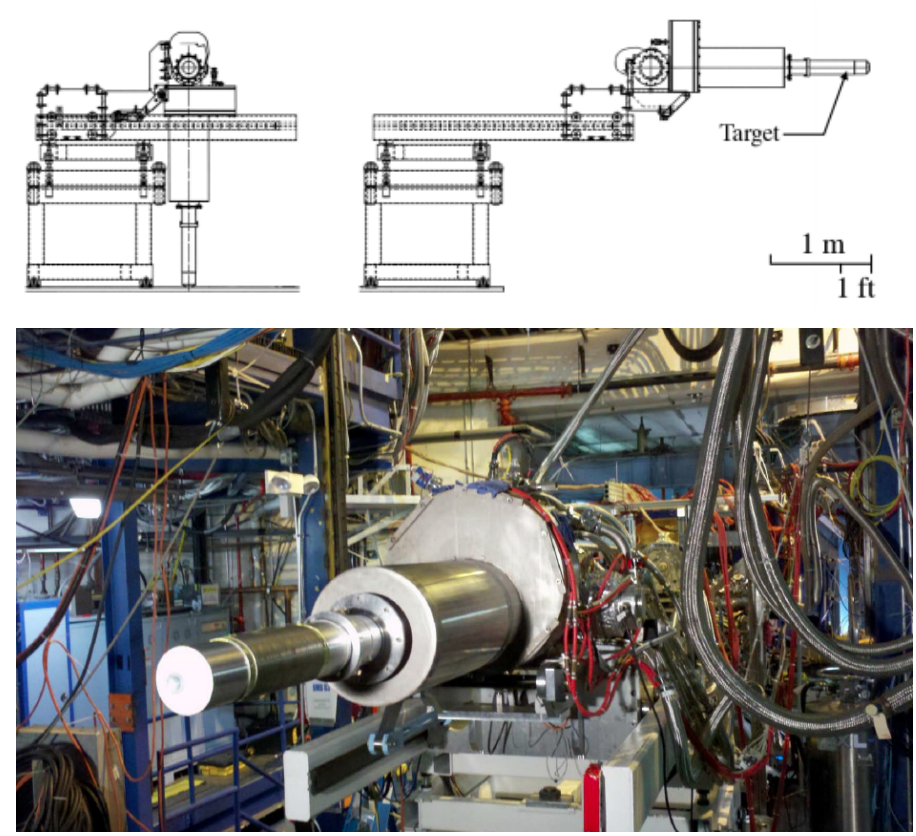

Figure 3.21: Left: The IBC in transfer configuration (vertical) [47]. Left: The IBC in the experimental configuration (under the beam, horizontal). 


\subsection{The HD-ice Target production}

In the following sections we will describe the typical sequence of operations necessary to create a frozen-spin polarized HD target and to transport it the In-Beam Cryostat located in Hall-B for the g14 experiment.

\subsubsection{Condensing the HD gas}

The very first step for the production of an HD target consisted in the condensation of the HD gas in the target cell. We remind that the target cell, whose shell was made of pCTFE, was fixed to a copper ring, possessing an interior left-handed thread and and exterior right-handed thread, through which the cell could be unscrewed from one cryostat and screwed it into another one. An empty target cell was installed on the end of a capillary tool and lowered into the cold-bore of the Production Dewar (PD) (see section 3.6.1)

The target cell was cooled down to a temperature just above the triple-point temperature of HD ( $\mathrm{T}=16.6 \mathrm{~K})$, and distilled HD gas (see section 3.3.1) was condensed through the capillary and into the target cell. The HD gas contained small amounts of $\mathrm{H}_{2}$ and $\mathrm{D}_{2}$ impurities with the optimal concentrations needed for the polarization process (as previously explained in section 3.3).

Once the target cell has been filled with liquid HD the temperature was lowered down, creating a solid HD crystal. The cell was then lowered to the $4 \mathrm{~K}$ copper threads of the PD where the capillary tool is removed.

\subsubsection{Thermal equilibrium calibration}

The second stage was the calibration, consisting in the measurement of the target polarization at the thermal equilibrium. For this purpose the HD target was positioned within a set of crossed coils located inside the PD used for NMR studies [56]. The polarization is proportional to the area of a NMR measurement:

$$
\text { Polarization }=C \cdot \text { Area }
$$

The calibration procedure consisted of determining the constant $\mathrm{C}$ of the target cell, which can be found by knowing the expected polarization and by measuring the area of the signal at thermal equilibrium. Since the cells were different (having different amounts of HD an different number aluminum wires) the calibration was done fore every cell separately. The calibration was done at thermal equilibrium because this is the predictable known polarization [47]. The thermal equilibrium was reached within few relaxation times, typically (before aging) this was of the order of seconds for $\mathrm{H}$ and $10 \mathrm{~s}$ to $100 \mathrm{~s}$ of $\mathrm{D}$, depending on the concentration of impurities as well as the temperature and the magnetic field in the PD; consequently $\mathrm{H}$ and D quickly reached their respective thermal equilibrium polarizations. To reduce the 
systematic errors on the calibration constant, multiple NMR measurements have been made. Since the thermal equilibrium signals are relatively small (of the order of $0.01 \%$ ) several measurements have been done, typically 400 for $\mathrm{H}$ and 200 for $\mathrm{D}$; in particular the thermal equilibrium signal for $\mathrm{D}$ is smaller but the $\mathrm{H}$ signal could be used to calibrate $\mathrm{D}$ since the ratio between the two signals is well-known.

\subsubsection{Transferring the target to the Dilution Fridge}

The HD target was extracted from the Production Dewar by using the Transfer Cryostat and transferred to the Dilution Fridge. A schematic for this operation shown in Figure 3.22. The entire process usually took about 10 hours.

The first step for extracting the cell was to connect the Transfer Cryostat to the Production Dewar. The Transfer Cryostat tube was lowered until it reached the cell, then the tube was rotated counterclockwise to screw the TC cold head into the cell copper ring and to unscrew the cell from the cryostat. This rotation had to be done very slowly because the friction when screwing and unscrewing could result in a quick heat up of the cell.

Once the cell was unscrewed from the Production Dewar it was lift up in the Transfer Cryostat; in the meanwhile the Dilution Fridge's transfer magnet was switched on ensuring a continuos magnetic field coverage. At this point the TC could be connected to the Dilution Fridge, and the cell was lowered to the bottom of this new cryostat.

Note that the Production Dewar and the Dilution Fridge were arranged in pits, as seen in Figure 3.22, so that their attachment points were at a common level.

Once the target cell was fully engaged into the cold-bore, continued clockwise rotation screwed the cell into the Dilution Fridge and, simultaneously, unscrewed the TC coldhead from the target copper ring.

\subsubsection{Polarization of the HD-ice Target}

As soon as the three cells (being three the maximum number that the DC cryostat could contain, (see section 3.6.3)) have been transferred to the Dilution Fridge the polarization process could be started. As we saw in section 3.3 the HD-ice target was polarized by using the brute force method which we remark it means raising the magnetic field to $15 \mathrm{~T}$ and lowering the temperature to $10 \mathrm{mK}$. Stabilizing the temperature down to $10 \mathrm{mK}$ requires several days because of the heat released while the HD polarizes and $\mathrm{J}=1 \mathrm{H}_{2}$ and $\mathrm{D}_{2}$ species decay to their ground states. This means that the optimal thermal equilibrium polarization condition cannot be achieved immediately. The polarization process is more efficient when the relaxation time is short, in fact the longer is the relaxation time the longer it takes to reach the thermal equilibrium. Since the relaxation time starts to increase as soon as the HD is condensed, all cells must be transferred quickly. 


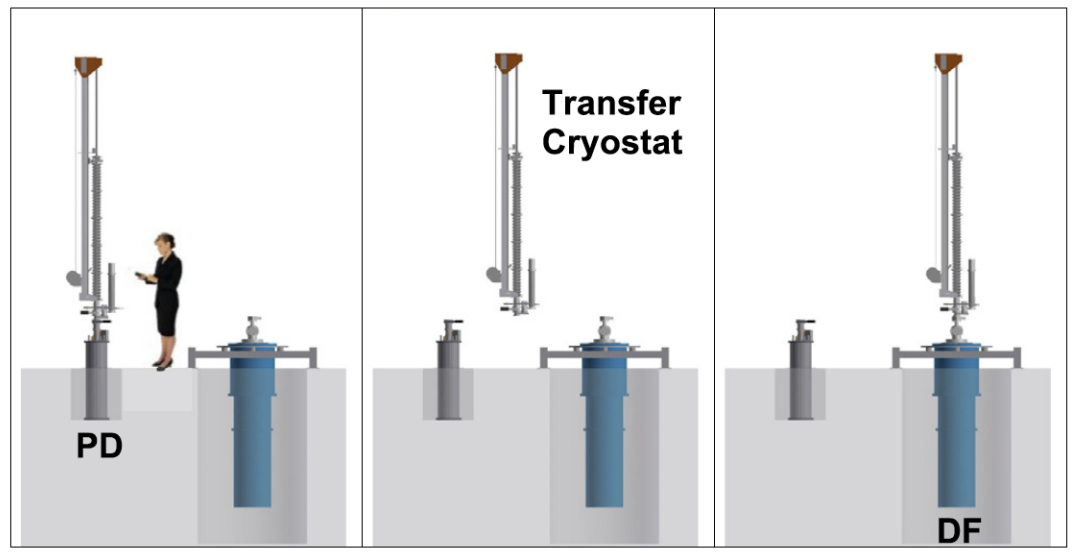

Figure 3.22: Schematic of the stages for the HD-ice target [46]

The whole process required about one week. The cell remains in the DF polarizing and aging for about three months.

\subsubsection{Measuring the HD-ice Target polarization}

After polarization, the target were extracted from the Dilution Fridge and inserted back into the Production Dewar to measure the polarization of $\mathrm{H}$ and $\mathrm{D}$. Target transfer operation proceeded analogously as described in section 3.7.3, but even more care is needed not to loose polarization during transfer. If the measured polarization value was satisfactory, its stability was monitored for a couple of hours; this guaranteed that the relaxation times for $\mathrm{H}$ and $\mathrm{D}$ were long enough for the use of the target in the experiment. Conversely, if the relaxation time of $\mathrm{H}$ and/or of $\mathrm{D}$ was found to be short, which would have meant that the initial relaxation time was to short and that the target did not age long enough; the cell could be transferred back to the DF to continue its aging and increase in this way the polarization level.

\subsubsection{Transporting the HD-ice Target to Hall B}

The transportation of the cell from the hd-ice lab to the hall $b$ was done with a truck . Once the cell was installed to the IBC, it remains there for the duration of the period assigned to that specific target. After that the cell was transferred back to the PD or the SD and transported back to the HDice lab. 


\subsubsection{Measuring the final HD-ice Target polarization}

The polarization values of the $\mathrm{H}$ and $\mathrm{D}$ were measured again after the end of datataking, to compare their current values with the initial one and with the thermal equilibrium measurement. At this point the remaining gas could be used for further characterization studies, as seen in sections 3.4 and 3.4.3.

\subsubsection{HD gas storage}

The HD can be retrieved by evaporating it in the PD. In order to do that the needle valve of the $\mathrm{PD}$ was closed to raise the temperature; when the valve was closed the VariTemp space run out of liquid helium and the temperature of the cold-finger increased above $50 \mathrm{~K}$, being $16 \mathrm{k}$ the boiling point of the $\mathrm{HD}$ at $1 \mathrm{~atm}$. Then the gas was pumped out of the cold finger by condensing it in a cold trap slowly lowered in a liquid helium supply dewar. After condensing and evaporation back and forth into smaller and smaller volume the HD gas can be stored in a tank with minimum gas loss. 


\section{Chapter 4}

\section{$\gamma N \rightarrow \pi^{+} \pi^{-} N$ event selection from the g14 data}

In this chapter we will describe the procedure used to identify the following reactions:

a) $\gamma \mathrm{p} \rightarrow \pi^{+} \pi^{-} \mathrm{p}$

b) $\gamma \mathrm{p}(\mathrm{n}) \rightarrow \pi^{+} \pi^{-} \mathrm{p}(\mathrm{n})$

c) $\gamma \mathrm{n}(\mathrm{p}) \rightarrow \pi^{+} \pi^{-} \mathrm{n}(\mathrm{p})$

The reactions a) and b) occur on free and quasi-free proton target. In this analysis the same selection criteria will be applied for the two reactions on the proton, regardless of the initial target momentum.

The special case where the two pions are coming from the strong decay of the $\rho$ vector meson, will be treated separately in chapter 5 for the reaction $\gamma \mathrm{p} \rightarrow \rho^{0} \mathrm{p}$.

\subsection{Running condition}

The data for the g14 experiment were taken between November 2011 and May 2012 in the experimental Hall B at the Thomas Jefferson National Laboratory (JLab) (see chapter 2).

During the g14 run different experimental settings were used, varying the energy of the incident electron beam, the beam current, the polarity and magnitude of the torus magnetic field and the type of polarization of the photon beam, which could be either circular or linear. Three different polarized HD frozen-spin targets were used, which had different polarization values for the Hydrogen and Deuterium.

These different experimental settings resulted in seventeen different periods, that is group of runs taken with the same experimental conditions.

In tables 4.1 and 4.2 the seventeen periods are listed along with the experimental 


\begin{tabular}{|l|l|l|l|l|l|l|}
\hline Period name & Beam Energy & Run Range & Date Range & Torus Current & Target Cell & Target Pol. \\
\hline Silver 1 & $2280.96 \mathrm{MeV}$ & $68021-68092$ & $12 / 1-12 / 6$ & $+1920 \mathrm{~A}$ & 21 a & $+\mathrm{D}(26.5 \%)+\mathrm{H}(14.8 \%)$ \\
\hline Silver 2 & $2280.96 \mathrm{MeV}$ & $68094-68176$ & $12 / 6-12 / 11$ & $+1920 \mathrm{~A}$ & 21 a & $+\mathrm{D}(21.7 \%)-\mathrm{H}(12.5 \%)$ \\
\hline Silver 3 & $2280.96 \mathrm{MeV}$ & $68188-68230$ & $12 / 12-12 / 16$ & $-1500 \mathrm{~A}$ & 21 a & $+\mathrm{D}(21.6 \%)+\mathrm{H}(0.3 \%)$ \\
\hline Silver 4 & $2257.75 \mathrm{MeV}$ & $68232-68305$ & $12 / 16-1 / 4$ & $-1500 \mathrm{~A}$ & 21 a & $-\mathrm{D}(18.0 \%)-\mathrm{H}(0.6 \%)$ \\
\hline Silver $5 \mathrm{a}$ & $2257.75 \mathrm{MeV}$ & $68335-68526$ & $2 / 4-1 / 19$ & $-1500 \mathrm{~A}$ & 21 a & $-\mathrm{D}(18.0 \%)-\mathrm{H}(0.6 \%)$ \\
\hline Silver $5 \mathrm{~b}$ & $2257.75 \mathrm{MeV}$ & $68533-68769$ & $1 / 19-2 / 5$ & $-1500 \mathrm{~A}$ & 21 a & $-\mathrm{D}(16.3 \%)-\mathrm{H}(0.9 \%)$ \\
\hline Gold 2 & $2541.31 \mathrm{MeV}$ & $69227-69364$ & $4 / 10-4 / 18$ & $-1500 \mathrm{~A}$ & $19 \mathrm{~b}$ & $+\mathrm{D}(26.9 \%)+\mathrm{H}(27.6 \%)$ \\
\hline Gold 3 & $2541.31 \mathrm{MeV}$ & $69365-69369$ & $4 / 19-4 / 19$ & $-1500 \mathrm{~A}$ & $19 \mathrm{~b}$ & $+\mathrm{D}(16.2 \%)+\mathrm{H}(18.9 \%)$ \\
\hline
\end{tabular}

Table 4.1: The dataset of the g14 experiment listed according to different parameters of the experiment such as the electron beam energy, torus current and the target polarization. The periods in this list are all associated with circularly-polarized photon beam. Periods names silver and gold are associated with two distinct HD targets.

\begin{tabular}{|c|c|c|c|c|c|c|}
\hline Period name & Beam Energy & Run Range & Date Range & Torus Current & Target Cell & Target Pol. \\
\hline Last tgt 3 & $1800 \mathrm{MeV}$ & $69476-69543$ & $5 / 3-5 / 9$ & $-1500 \mathrm{~A}$ & $22 \mathrm{~b}$ & $+\mathrm{D}(24.5 \%)+\mathrm{H}(22.9 \%)$ \\
\hline Last tgt 4 & $1800 \mathrm{MeV}$ & 69544-69578 & $5 / 9-5 / 12$ & $-1500 \mathrm{~A}$ & $22 \mathrm{~b}$ & $-\mathrm{D}(17.0 \%)+\mathrm{H}(18.1 \%)$ \\
\hline Last tgt $2 \mathrm{a}$ & $2000 \mathrm{MeV}$ & 69398-69419 & $4 / 27-4 / 28$ & $-1500 \mathrm{~A}$ & $22 \mathrm{~b}$ & $+\mathrm{D}(26.9 \%)+\mathrm{H}(27.6 \%)$ \\
\hline Last tgt $2 b$ & $2000 \mathrm{MeV}$ & $69420-69474$ & $4 / 29-5 / 3$ & $-1500 \mathrm{~A}$ & $22 \mathrm{~b}$ & $+\mathrm{D}(24.5 \%)+\mathrm{H}(22.9 \%)$ \\
\hline Last tgt 5 & $2000 \mathrm{MeV}$ & $69583-69616$ & $5 / 12-5 / 15$ & $-1500 \mathrm{~A}$ & $22 \mathrm{~b}$ & $-\mathrm{D}(17.0 \%)+\mathrm{H}(18.1 \%)$ \\
\hline Gold 1 & $2200 \mathrm{MeV}$ & 69194-69225 & $4 / 6-4 / 9$ & $-1500 \mathrm{~A}$ & $19 \mathrm{~b}$ & $+\mathrm{D}(26.9 \%)+\mathrm{H}(27.6 \%)$ \\
\hline Gold 4 & $2200 \mathrm{MeV}$ & 69370-69372 & $4 / 21$ & $-1500 \mathrm{~A}$ & $19 \mathrm{~b}$ & $+\mathrm{D}(16.2 \%)+\mathrm{H}(18.9 .6 \%)$ \\
\hline Last tgt 1 & $2200 \mathrm{MeV}$ & $69375-69397$ & $4 / 25-4 / 27$ & $-1500 \mathrm{~A}$ & $22 \mathrm{~b}$ & $+\mathrm{D}(24.5 \%)+\mathrm{H}(26.1 \%)$ \\
\hline Last tgt 6 & $2200 \mathrm{MeV}$ & $69618-69636$ & $5 / 16-5 / 17$ & $-1500 \mathrm{~A}$ & $22 \mathrm{~b}$ & $-\mathrm{D}(17.0 \%)+\mathrm{H}(18.1 \%)$ \\
\hline
\end{tabular}

Table 4.2: The dataset of the g14 experiment listed according to different parameters of the experiment such as the electron beam energy, torus current and the target polarization. The periods in this list are all associated with linearly-polarized photon beam. Period names gold and last are associated to two distinct HD targets.

settings used in each period. The tables are separated according to the type of the photon beam polarization: table 4.1 refers to data taken with circularly-polarized photon, table 4.2 refers to data taken with linearly-polarized photon. In this work, only datasets from table 4.1 will be considered.

\subsection{Organization of the g14 data: collection, calibration and cooking}

The event files collected during on-line data acquisition were stored on the JLab tape silo system; these files contained informations about the detector ADC and TCD readouts at the time the event was recorded in CLAS, and are called raw data files. These data were organized in sequence of banks which are accessed and 
managed using a Fortran 77 dynamic memory allocation system structure known as BOS (Bank Operating System) [58], [59]. To convert these raw data into interpretable physical quantities such as particle IDs, positions, angles and four-momenta, a multi-step procedure was followed.

First, after the experiment finished running, the raw, unprocessed, data are calibrated; each subsystem had a number of calibration constants, associated to it, and a calibration had to be performed for every detector. A precise order in the detectors calibration had to be followed and the calibration cycle iterated several times, since the measurements from different systems are not independent.

In the meanwhile the quality of the reconstructed data was monitored: if the result was not satisfying, the constant were recalculated and successive passes of reconstruction were performed until the quality of monitored quantities could be considered acceptable.

The author was responsible for the timing calibration of the forward electromagnetic calorimeter, which will be described in the next section.

When adequate constants have been obtained, the data were processed ("cooked") using the CLAS reconstruction package. In the final "cooked" files, the BOS format raw and cooked banks are both present.

The cooked data are converted to a ROOT file format; ROOT is an object oriented framework used in large-scale analysis developed at CERN, which allows for the utilization of standard libraries for data analysis procedure [60].

At this point, the different user-customised off-line analysis could start. For this thesis work the ROOTBEER package (ROOT Bank Event Extraction Routines) developed by the Glasgow University was used [61].

In order to optimize the disk space requirements for the off-line analysis, a filtering package was used on the reconstructed data set, resulting in a set of reduced files called "skimmed files" for each physical channel of interest, which will contain a reduced set of banks. The flow-chart showing this procedure it's shown in Figure 4.1, for both experimental and simulated data.

\subsubsection{The forward electromagnetic calorimeter timing calibration}

The final step of every calibration cycle was the timing calibration of the forward electromagnetic calorimeter.

This calibration depended very much on the quality of the Time-Of-Flight calibration. As we saw in section 2.5.5, the main task of the electromagnetic calorimeter was the detection and energy determination of neutral particles and, in particular, the discrimination between neutrons and photons, based on their time-of-flight information. It follows that the TDC timing calibration was crucial to perform such a separation. The calibration was performed using electrons and charged pions, for which the time of flight could be easily established. The time-of-flight from the elec- 


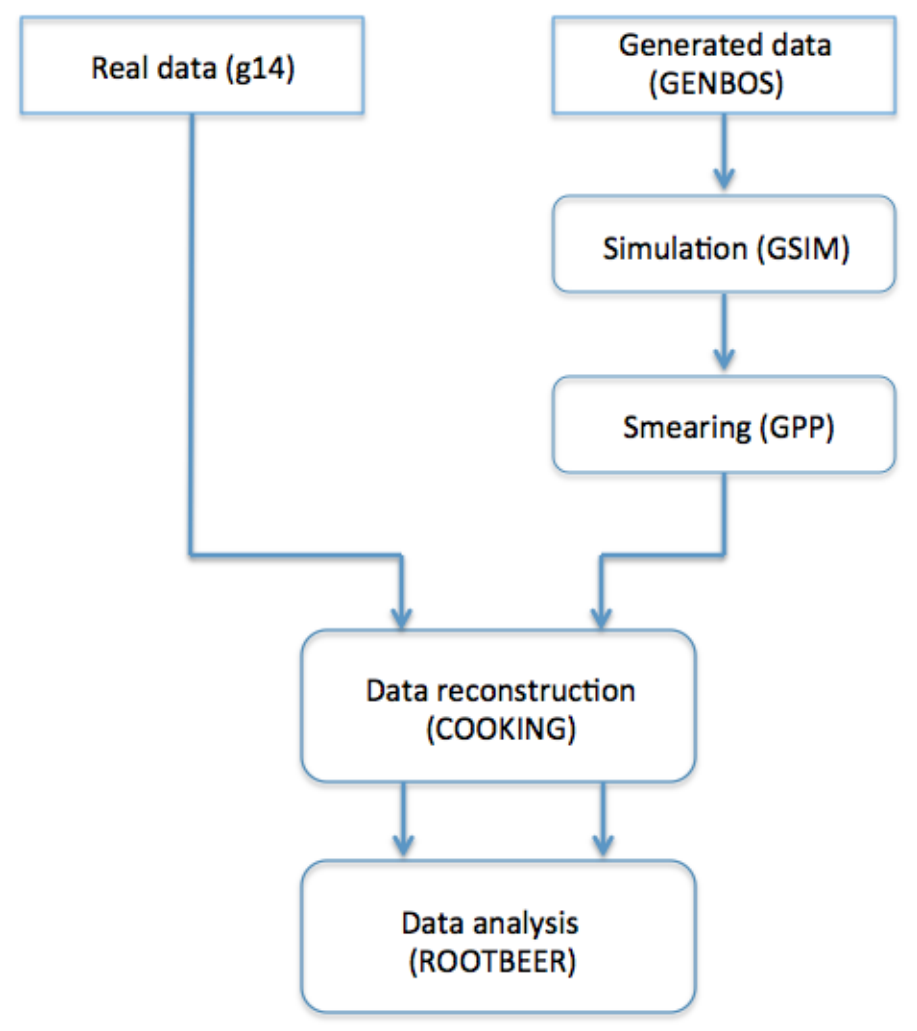

Figure 4.1: Flow chart of the program software for simulated (right) and measured (left) data. 
tromagnetic calorimeter can be evaluated using a semi-empirical model, which uses a set of five parameters denoted $a_{k}$ where $\mathrm{k}=0, \ldots, 4$ :

$$
T_{\text {model }}=a_{0}+a_{1} T D C+a_{2} \frac{1}{\sqrt{A D C}}+a_{3} l^{2}+a_{4} l^{3}
$$

where:

- $a_{0}$ is the constant terms and includes all constant times (i.e. delays due to cable length)

- $a_{1} T D C_{i}$ is the TDC conversion term in ns.

- $a_{2} \frac{1}{\sqrt{A D C_{i}}}$ is the time - walk correction term

- $a_{3} l_{i}^{2}$ and $a_{4} l i^{3}$ are the light attenuation terms

For each PMT, indexed by $\mathrm{j}$, we have a set of $a_{j, k}$ parameters; the aim of the calibration process was to adjust them so that $T_{\text {model }}$ matched as close as possible the time-of-flight measured from the scintillator counters which will be indicated by $T_{\text {measured }}$ in the following. In order to find the best calibration constants, a chisquare minimization was performed, to compare the timing from the time-of-flight detectors to that of the EC described by the five parameters model $T_{\text {model }}$ :

$$
\chi_{j}^{2}=\sum_{i=1}^{N_{j}} \frac{\left|T_{s c}^{\prime}-T_{\text {model }}\right|^{2}}{\sigma_{j, i}^{2}}
$$

where the index $\mathrm{i}$ is on the number of hits recorded on the PMTs and $N_{j}$ is their total number, $\sigma_{j, i}$ denotes the time resolution due to statistical and systematical errors. The term $T_{s c}^{\prime}$ corresponds to:

$$
T_{s c}^{\prime}=T_{s c}+\frac{d i s t_{e c-s c}}{\beta}(n \cdot v)
$$

where $T_{s c}$ is the time-of-flight measured from the scintillator counter subsystem, $d_{i s t_{e c} c}$ is the perpendicular distance between the scintillator counter and the electromagnetic calorimeter and $(n \cdot v)$ is a factor which takes into account the actual path of the tracks. In Figures 4.2 and 4.3 some monitoring histograms for the calibration of the electromagnetic calorimeter are shown. In order to study the stability of the calibration of the electromagnetic calorimeter, we studied the trend of the five parameters as a function of the run number and the strip number, as shown in Figure 4.4. For convenience, the strip numeration goes from 1 to 70 , where the strips from 1 to 36 belong to the inner part of the calorimeter whereas those from 37 to 70 belong to the outer part. One can see that for all the $a_{i}$ parameters the trend is quite stable along both the strip and the run number, indicating the good quality of the electromagnetic calorimeter calibration. This quite flat trend is interrupted only in correspondence of few regions that we identified as associated to non working strips. 

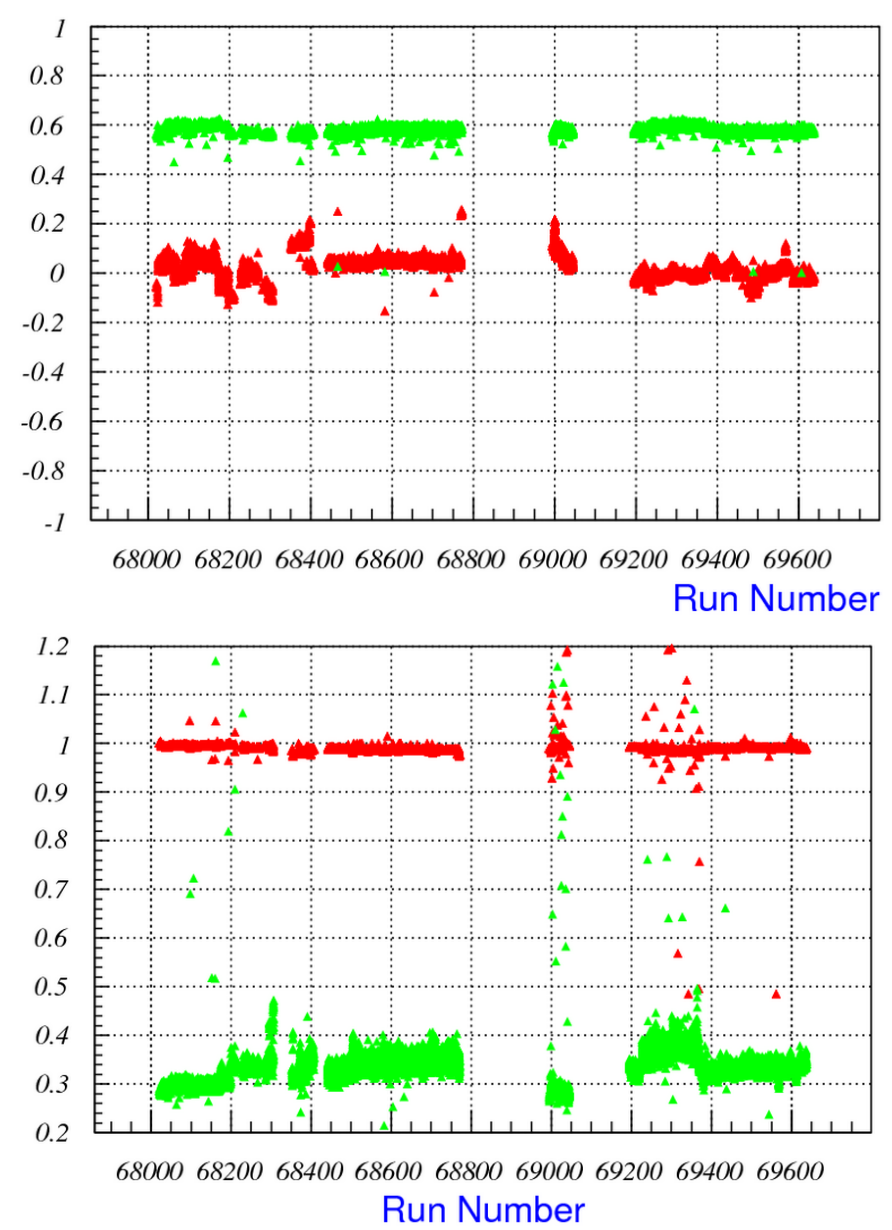

Figure 4.2: Top: Mean of the difference between the calibrated time-of-flight measured from the electromagnetic calorimeter and the time-of-flight measured from the scintillator counters subsystem; bottom: calibrated $\beta$ for photons; red triangles represent the mean values, green triangles represent the sigma. 

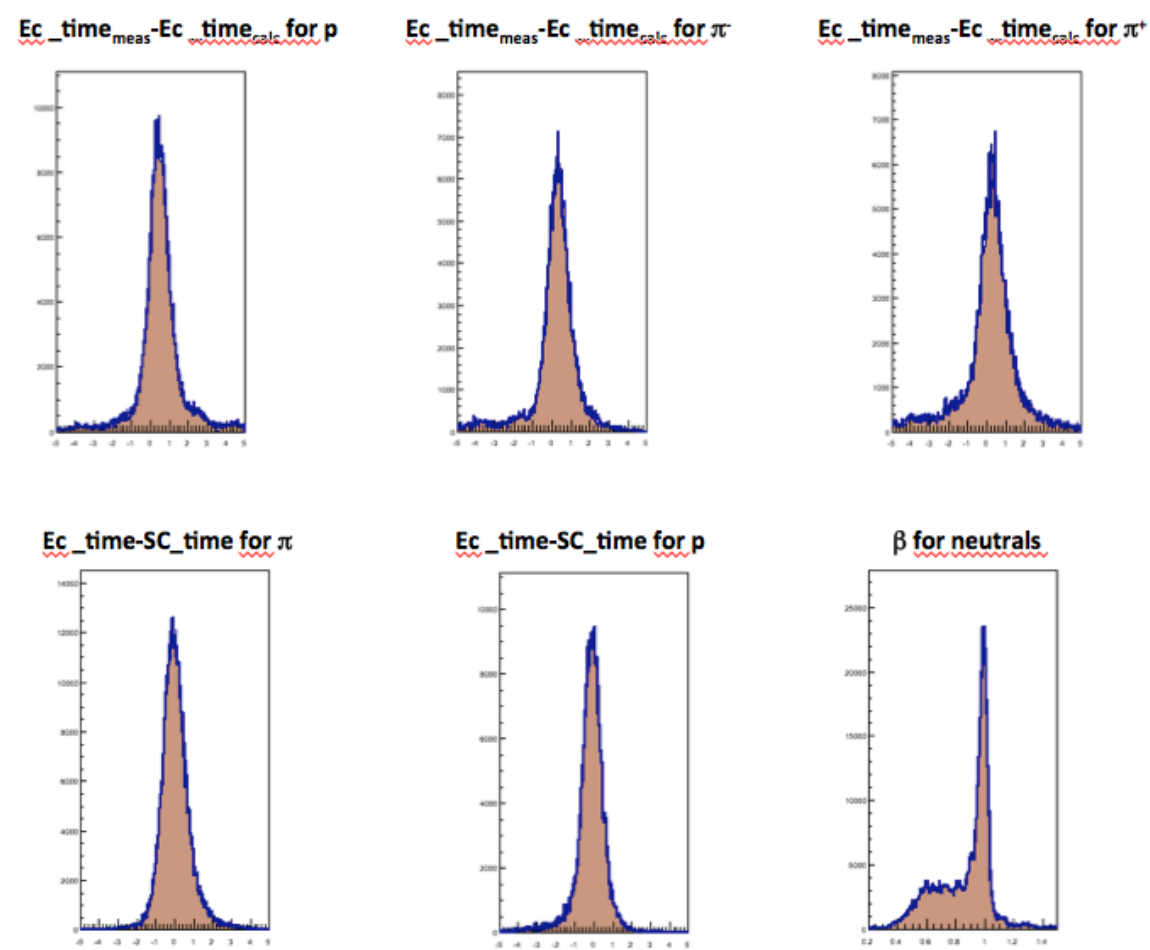

Figure 4.3: Top: difference between the calibrated time-of-flight measured from the electromagnetic calorimeter and the calculated time-of-flight; starting from upper left: the distributions for protons, $\pi^{+}$and $\pi^{-}$. The difference between the calibrated time-of-flight measured from the electromagnetic calorimeter and the time-of-flight measured from the scintillator counters subsystem is plotted in the bottom line; the last plot on the right shows beta for neutral particles. 


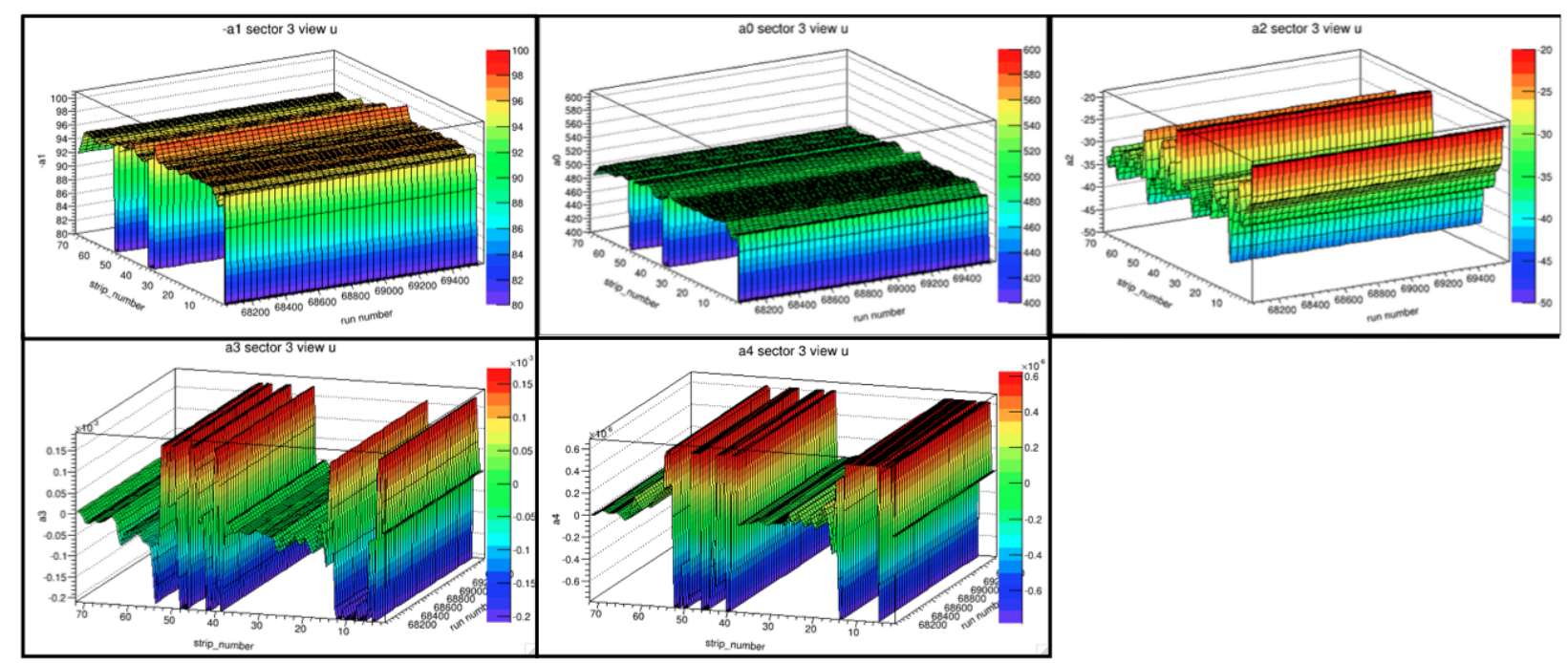

Figure 4.4: The five parameter $a_{i}$ as a function of the run number and strip number; on the $\mathrm{x}$-axis is the run number, on the $\mathrm{y}$-axis the strip number.

\subsubsection{Simulation chain}

The simulation chain program starts with a dedicated even generator, GENBOS [62], developed for CLAS. An event is generated among 37 possible reactions on the nucleon for photon energies from pion production threshold up to $10 \mathrm{GeV}$. For each hadronic event the program selects one or more reaction channels, generates the kinematic quantities of final particles according to the tabulated cross sections for two-body channels or though the CERN routine GENBOD for multi-body channels, checking for conservation of four-momenta and takes into account the $\Delta$ and $\rho$ decays, if needed. Once the event has been generated, the interaction of each particle with the CLAS detector is simulated with a package GEANT based (GSIM), which simulates the geometry of each element making up the CLAS detector. All the simulation options are written in a text file called ffread card, where a set of FORTRAN77 subroutines are configured using parameters appropriate for the g14 experiment. The output of GSIM is still unrealistic, being the result of simulating the CLAS detector working at full efficiency. For this reason, the output of GSIM is fed into GPP, the GSIM Post-Processor, which simulates inefficiencies of the CLAS detector, taking into account the subdetectors resolution. For example, GPP removes hits from known dead detection elements, such as dead TOF photomultipliers or dead wires in the DC system. It also smears the timing output from the Scintillator Counters and the drift chambers according to a Gaussian distribution, consistent with the overall resolution of the various detector components. The output from GPP is 
then analyzed by RECSIS, the same program also used for the reconstruction of the experimental data. RECSIS is controlled by a tcl file, which allows to handle simulated data with a specific set of instructions, appropriate for the g14 experiment. Finally, as it happens for real data, the output from RECSIS is analyzed with the ROOTBEER package, customized by the user for the reaction of interest.

\subsection{Data banks and first data skimming}

As explained in the previous section, the information for each event has been organized in data banks; these latter contain the properties of the particles involved in the reaction and the information about the detector hits. The first important information on the particles, determined during the cooking, was the particle's identification, associated to a number named PID. This quantity allows to filter only events containing the final state particles of interest in the analysis. In this work, the PID contained in the GPID bank was used [63]. The momentum of detected particle is measured using the tracking of the trajectories bent by the torus field in the drift chambers; sequentially trial values of $\beta$, the particle velocity in units of light speed, are calculated for all possible particle identities. Each one of the possible identities is tested comparing the difference between the trial value of $\beta$ and its measured value from time-of-flight information. The particle is assigned the identity that provides the closest trial value to the empirically measured one.

Other information contained in the GPID bank and used in this analysis, is: fourvectors, charge, $\beta$ of the particle both from the scintillator counter and the drift chambers information, the vertex time of the particle's track, particle masses, the incident photons energy and time.

In addition to the GPID, the following banks are used in this thesis work:

- TAGR: Contains informations recorded by the tagger system: the energy of all recorded photons, the raw and the corrected photon timing, taking into account the RF phase.

- TBER: Contains the tracking resolution errors associated with the tracking measurements of the particles on the event.

- MVRT: Contains the vertex information for the event.

- ECHB: Contains information about the relevant quantities associated with hits in the electromagnetic calorimeter.

In order to speed up the analysis and to deal with a reduced set of data, many banks were dropped and different topologies were selected. This filtering was done by placing loose initial requirements based on the PID, the $\mathrm{z}$ coordinate of the vertex and the $\beta$ of the particle (from the Drift Chambers) as determined during the cooking. 
The skimmed set of data used in the present analysis are the n2pi and 2pos1neg skims.

The $n 2 p i$ skim contains at least one neutral particle and at least two charged pions, the 2pos1neg skim contains 2 positive tracks, one negative track and any number of neutrals.

The n2pi skim requires:

- GPID[track number] $=8,9,13$.

- number of protons $=0$

- number of neutrals $>0$

- number of positive pions: $0<\pi^{+}<3$

- number of negative pions: $0<\pi^{-}<3$

- the distance of z vertex coordinates for at least one $\pi^{+}$and $\pi^{-}$pair $<2.0 \mathrm{~cm}$

- An additional window of allowed values for $\beta$, calculated as:

$$
\beta=\frac{p}{\sqrt{p^{2}+m_{P D G}^{2}}}
$$

is set, using a wide mass interval $\left(m_{\min }, m_{\max }\right)$ around the particle mass value, as reported from the Particle Data Group [7].

- $\beta_{\min }=\frac{p}{\sqrt{p^{2}+0.3^{2}}}-0.03$ and $\beta_{\max }=\frac{p}{\sqrt{p^{2}+0.05^{2}}}+0.03$;

The 2pos1neg skim requires:

- number of positive tracks $=2$

- number of negative tracks $=1$

- any neutrals

- the distance of $\mathrm{z}$ vertex coordinates for all tracks $<2.0 \mathrm{~cm}$

- Acceptance windows for particle velocity $\beta$ is defined as follows for different charged particles:

- Pions: $\beta_{\min }=\frac{p}{\sqrt{p^{2}+0.3^{2}}}-0.03$ and $\beta_{\max }=\frac{p}{\sqrt{p^{2}+0.05^{2}}}+0.03$

- Kaons: $\beta_{\min }=\frac{p}{\sqrt{p^{2}+1.1^{2}}}-0.06$ and $\beta_{\max }=\frac{p}{\sqrt{p^{2}+0.8^{2}}}+0.06$ 
- Protons: $\beta_{\min }=\frac{p}{\sqrt{p^{2}+0.6^{2}}}-0.05$ and $\beta_{\max }=\frac{p}{\sqrt{p^{2}+0.4^{2}}}+0.05$

Since for this work the reactions of interest are $\gamma n(p) \rightarrow \pi^{+} \pi^{-} n(p), \gamma p \rightarrow \pi^{+} \pi^{-} p$ and $\gamma p(n) \rightarrow \pi^{+} \pi^{-} p(n)$, the very first requirements imposed by the author were the presence in the final state of exactly 1 neutron, $1 \pi^{+}, 1 \pi^{-}$or 1 proton, $1 \pi^{+}, 1 \pi^{-}$for the two channels, respectively.

\subsection{Applied corrections to the data}

For the g14 experiment some data corrections were developed by our team and applied in the present work; the corrections will be described in the next sections.

\subsection{Kinematic Fitting}

The kinematic fitting is an iterative procedure, based on the least squares method; it uses physical constraints of energy and momentum conservation, along with the knowledge of detector uncertainties, to improve the precision of measured quantities. The software used in this analysis, developed at the Carnagie Mellon Universities, performs the least squares fit using the Lagrange Multipliers method to impose the energy and momentum conservation. Details are shown in Appendix A.

Measured quantities may be written as:

$$
\vec{\eta}=\vec{y}+\vec{\epsilon}
$$

where $\vec{y}$ are the estimators variables, as given by the least squares fit and $\vec{\epsilon}$ are the set of the deviations needed to shift the observed values $\vec{\eta}$ to satisfy physical constraints.

In the ideal case, where the fit estimator $\vec{y}$ are close to the measured quantities $\vec{\eta}$, the shifts $\vec{\epsilon}$ should be normally distributed around zero. In order to check the $\vec{\epsilon}$ distribution at each iteration, the pulls at each iteration, defined as:

$$
z=\frac{\eta_{i t}-y_{i t}}{\sigma_{\eta_{i t}}^{2}-\sigma_{y_{i t}}^{2}}
$$

are examined. The pulls z measure the difference between the quantities $\eta$ and the estimators given by the fit, $y_{i t}$, relative to their errors $\sigma_{\eta_{i}}^{2}$ and $\sigma_{y_{i}}^{2}$.

The procedure is iterated until the pulls distributions are reasonably close to the ideal Gaussian distribution, centered around zero, with $\sigma^{2}=1$, for each event and each physical quantity.

The goodness of the fit with respect to the constraint hypothesis, is given in terms of confidence level (CL), which gives the probability of error if the hypothesis is discarded. Small values of CL correspond to events which do not satisfy the physical hypothesis and can be discarded. 


\subsubsection{CLAS tracking parameters}

There are three coordinate systems defined for the CLAS detector: the lab system, the sector system and the tracking system. In the lab system the $z_{l a b}$ axis is along the beam line, the $x_{l a b}$ axis passes through the center of sector 1, and the $y_{l a b}$ axis points vertically upwards, between sector 2 and 3. The lab system is the one used for physics analysis. The tracking coordinates in each sector are defined such that the $x_{\text {track }}$ axis lies along the beam line, the $y_{\text {track }}$ axis pass through the center of the sector and the $z_{\text {track }}$ axis is aligned with the average magnetic field direction in that sector. The track reconstruction in CLAS is carried out in the tracking system. The lab and track system are represented in Figure 4.5. The sector system is not discussed here, not being relevant for this theses.

The tracking coordinates are related to the lab coordinates by the following relations:

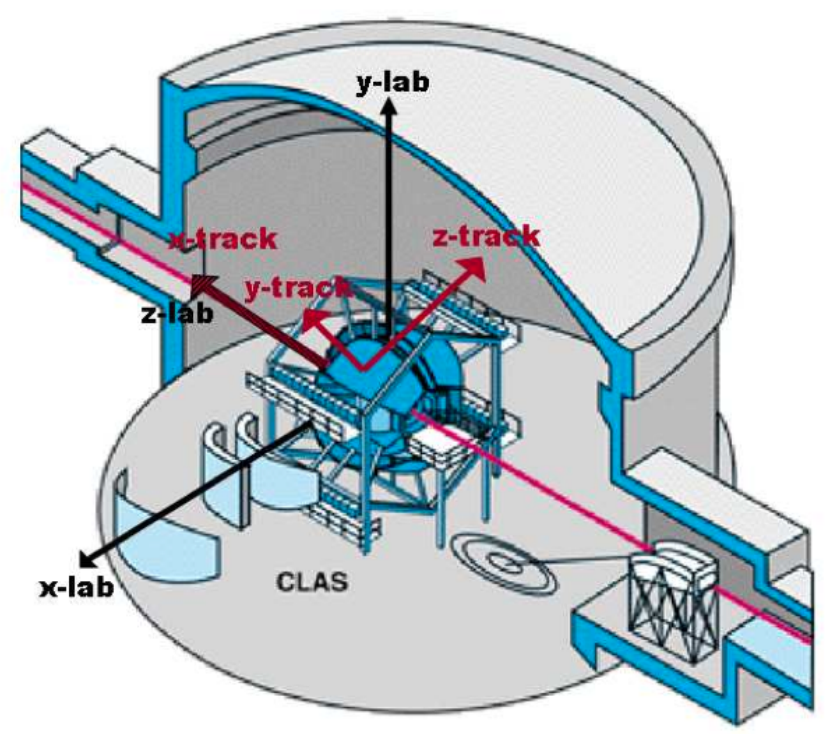

Figure 4.5: The lab coordinate system along with the tracking coordinates for sector 2 [67].

$$
\left(\begin{array}{c}
x_{\text {track }} \\
y_{\text {track }} \\
z_{\text {track }}
\end{array}\right)=\left(\begin{array}{c}
z_{\text {lab }} \\
\cos (\alpha) x_{l a b}+\sin (\alpha) y_{l a b} \\
-\sin (\alpha) x_{l a b}+\cos (\alpha) y_{l a b}
\end{array}\right)
$$

where $\alpha=\frac{\pi}{3}\left(N_{\text {sector }}-1\right)$ 
The track momenta may be reconstructed in terms of the ratio $\mathrm{q} / \mathrm{p}$, where $\mathrm{q}$ is the charge and $\mathrm{p}$ is the momentum magnitude, the dipolar angle relative to the sector's $\left(x_{\text {track }}, y_{\text {track }}\right)$ plane $\lambda$, and the angle in the sector's $\left(x_{\text {track }}, y_{\text {track }}\right)$ plane relative to the $x_{\text {track }}$ axis $\phi$. The momenta in the lab frame may be written in terms of these tracking parameters as:

$$
\left(\begin{array}{c}
p x_{l a b} \\
p y_{l a b} \\
p z_{l a b}
\end{array}\right)=\left(\begin{array}{c}
p(\cos (\lambda) \sin (\phi) \cos (\alpha)-\sin (\lambda) \sin (\alpha)) \\
p(\cos (\lambda) \sin (\phi) \sin (\alpha)+\sin (\lambda) \cos (\alpha)) \\
p(\cos (\lambda) \cos (\phi))
\end{array}\right)
$$

\subsection{Energy and Momentum Corrections}

Both the tagger and momentum corrections in this analysis were derived using the reaction $\gamma p \rightarrow p \pi^{+} \pi^{-}$and the kinematic fitting procedure described in the previous section. The procedure used to calculate the tagger and momentum corrections is the following. First the tagger corrections are obtained; these corrections are then applied to obtain the momentum corrections. Since the presence of a systematic offset in the momenta could affect the tagger corrections, an iterative approach must be used. For each iteration, we apply the previous iteration momentum corrections, then calculate the tagger correction. Next, we apply the new tagger correction and recalculate the momentum corrections. In the sections below the procedure used during a single iteration is reported.

\subsubsection{Energy Loss correction}

As charged particles travel from the target cell to the Drift Chambers, they lose part of their energy passing through the target material, the target cell walls, support structures, the beam pipe, the start counter and the air gap between the start counter and the Region 1 of the Drift Chambers. As a result, the reconstructed particle's momentum determined by the Drift Chambers is smaller then the particle's momentum at the production vertex. To account for this effect, the four-vectors of the final state particles were corrected event-by-event by using the eLoss software package [64].

The particle's four-momentum, as measured by the Region1 Drift Chambers, was used to track the particle back to the interaction vertex in the target cell; the different materials and the distances it travels are considered and the energy loss of the detected particle is calculated. Knowing the energy loss, the four-vector of the particles can be corrected. In Figure 4.6 the effect of the energy loss correction on the Missing Mass from the reaction $\gamma \mathrm{p} \rightarrow \pi^{+} \pi^{-}(\mathrm{p})$ is shown, where the blue histogram is shifted to a narrower red histogram, centered at the proton PDG mass after correction. Energy loss is the largest of the applied corrections and it is done before any kinematical fitting. 


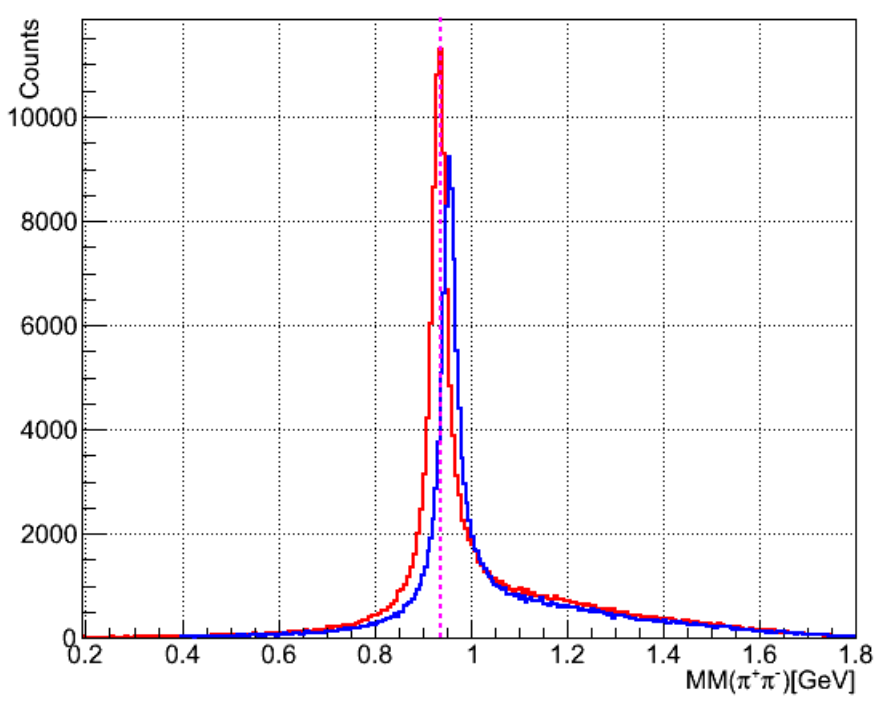

Figure 4.6: Missing Mass $M M\left(\pi^{+} \pi^{-}\right)$from the reaction $\gamma \mathrm{p}->\pi^{+} \pi^{-}$(p) before (blue histogram) and after (red histogram) energy loss correction. The Magenta line indicates the proton mass value from PDG. 


\subsubsection{Tagger correction}

Alignment issues in the photon tagger's focal plan lead to an inaccurate photon energy reconstruction from the raw tagger information. It is possible to obtain an empirical correction for this effect by studying the channel $(\gamma) \mathrm{p} \rightarrow \mathrm{p} \pi^{+} \pi^{-}$, where one proton, one $\pi^{+}$and one $\pi^{-}$in the final states were selected. The energy loss was applied before calculating the Tagger correction. The event is then kinematically fitted to the hypothesis $(\gamma) \mathrm{p} \rightarrow \mathrm{p} \pi^{+} \pi^{-}$, where the information on the measured photon energy is not used. Only events with a confidence level greater than $10 \%$ are used to determine the correction. For each event the kinematic fit estimate for the tagger correction is:

$$
\Delta E_{\text {gamma }}=E_{\gamma}^{k f i t}-E_{\gamma}^{\text {meas }}
$$

The two-dimensional histogram of the relative tagger corrections $\Delta E_{\gamma} / E_{\gamma}^{\text {meas }}$ for each tagger E-counter is shown in the upper part of Figure 4.7. For each E-counter the Gaussian means is extracted, as plotted in the lower part of Figures 4.7.

\subsubsection{Momentum correction}

Due to unknown variations in the magnetic field provided by the Torus Magnet and to inefficiencies and misalignments of the Drift Chambers, a momentum correction was necessary for the g14 run period. The channel $\gamma \mathrm{p} \rightarrow \mathrm{p} \pi^{+} \pi^{-}$was used to obtain empirical corrections for the reconstructed particles momenta. One $\pi^{+}$, one $\pi^{-}$and one proton were selected in the final state. The energy loss correction and the previous iteration's tagger correction are applied to all three final state particles Then the event is kinematically fitted [65],[66] sequentially to the three hypothesis: $\gamma \mathrm{p} \rightarrow \mathrm{p} \pi^{+}\left(\pi^{-}\right), \gamma \mathrm{p} \rightarrow \mathrm{p}\left(\pi^{+}\right) \pi^{-}$and $\gamma \mathrm{p} \rightarrow(\mathrm{p}) \pi^{+} \pi^{-}$. Using the formalism introduced in section 4.5, for each iteration we have a vector of measured quantities $\vec{y}^{T}=\left(p_{i 1}, \lambda_{i 1}, \phi_{i 1}, p_{i 2}, \lambda_{i 2}, \phi_{i 2}\right)$ and a vector of parameters $\vec{x}=\left(p_{x}, \lambda_{x}, \phi_{x}\right)$, where the indexes i1, i2 represent the considered particles for each fit and p, $\lambda, \phi$ are the tracking parameters, as described in section 4.5.1.

Only events for which the final confidence level is greater than $10 \%$ are used for determining the corrections. The kinematic correction are then computed in the following way:

$$
\begin{aligned}
& \Delta p_{x}=p_{x}^{k f i t}-p_{x}^{\text {meas }} \\
& \Delta \lambda_{x}=\lambda_{x}^{k f i t}-\lambda_{x}^{\text {meas }} \\
& \Delta \phi_{x}=\phi_{x}^{k f i t}-\phi_{x}^{\text {meas }}
\end{aligned}
$$

where $\mathrm{x}=\left(\mathrm{p}, \pi^{+}, \pi^{-}\right)$denotes the excluded particle. For example $\Delta_{p_{p}}$ was obtained using $p_{p}^{k f i t}$ from the kinematic fit to the $\gamma p \rightarrow(p) \pi^{+} \pi^{-}$reaction, whereas $\Delta_{p_{\pi^{-}}}$was 

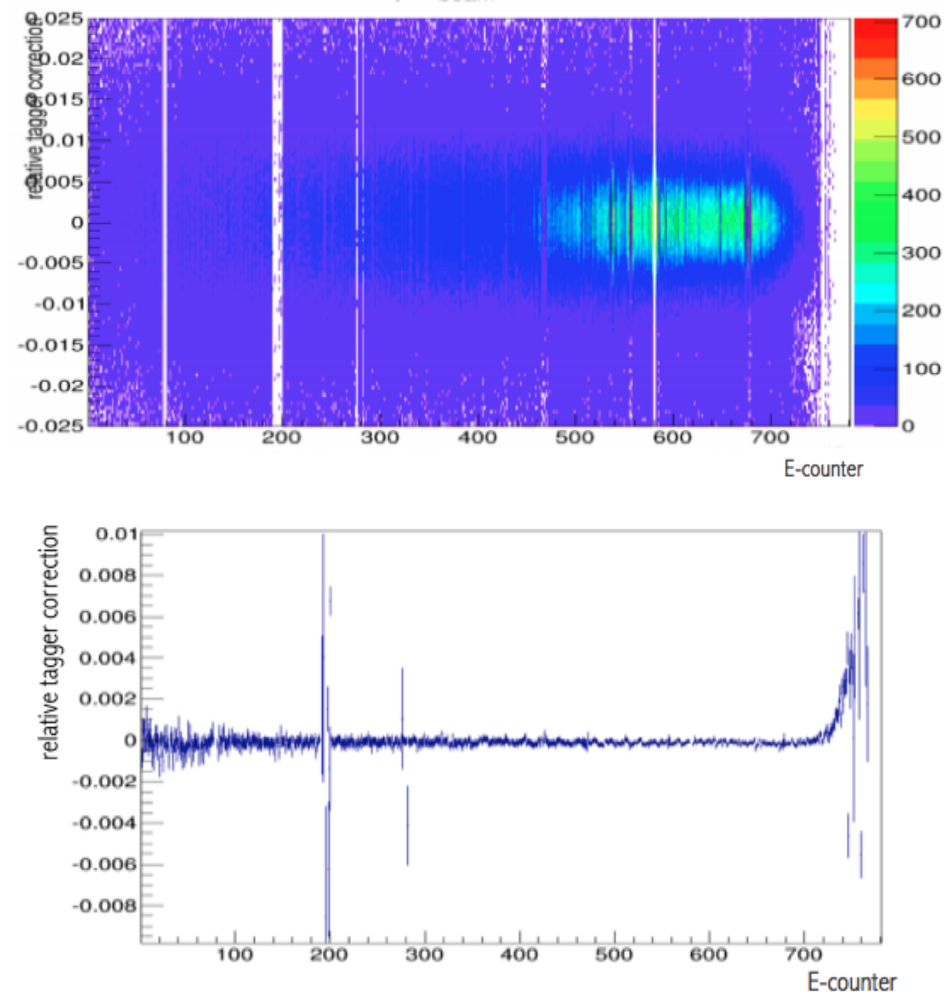

Figure 4.7: Tagger Corrections. Top: $\Delta E_{\gamma} / E_{\gamma}^{\text {meas }}$ vs E-counter for $(\gamma) p \rightarrow p \pi^{+} \pi^{-}$ events. Bottom: Gaussian mean extracted for each E-counter from top plot. 
obtained using $p_{p}^{k f i t}$ from the kinematic fit to the $\gamma p \rightarrow p \pi^{+}\left(\pi^{-}\right)$reaction.

Each quantity in formulas (4.10) is a function of the particle's momentum magnitude, orientation in the lab coordinates $\theta$ and $\phi$, charge and CLAS sector number. Therefore the corrections were obtained for 1080 bins $=6$ CLAS sectors $\times 15 \theta \times 12 \phi$. A correction can be calculated for the quantities (4.10) as a polynomial function of $\mathrm{p}$, in each bin; two-dimensional histograms $\Delta X_{q}$ vs $p_{q}$, where $X_{q}$ denotes one of the quantities in (4.10), are plotted; the momentum magnitude $\mathrm{p}$ is binned in equal sized $1 / \mathrm{p}$ bins, since the tracking is done in terms of $1 / \mathrm{p}$. The Gaussian means are extracted for each $\mathrm{p}$ bin and fit to a polynomial function of p. In Figure 4.8 an example of this procedure is shown, for the three tracking variables of the proton for the bin $($ sector $, \theta, \phi)=(1,4,5)$. Note that the momentum correction depends only on the particle charge and not on its type, for this reason the correction for proton and $\pi^{+}$is exactly the same.
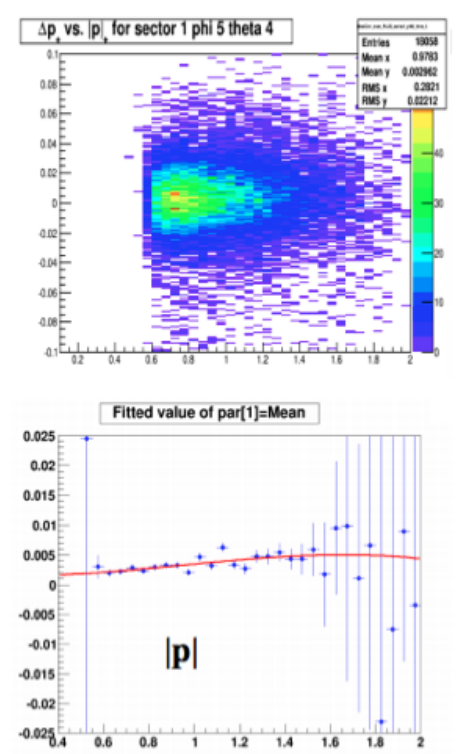
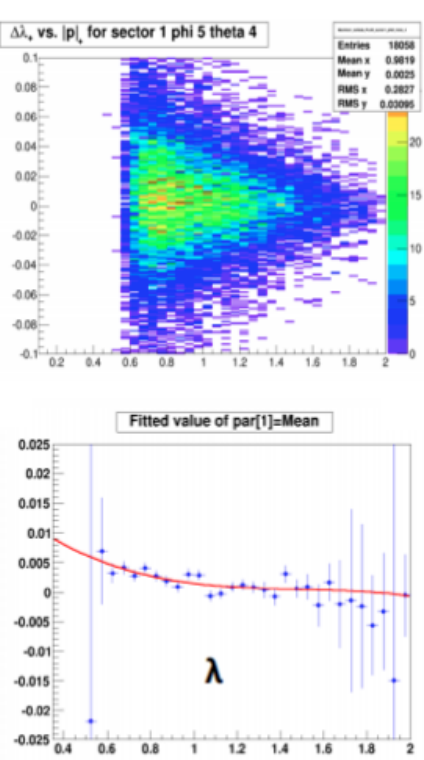
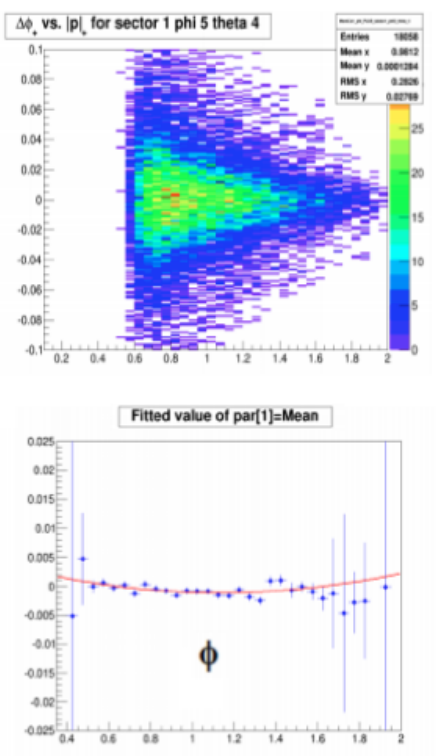

Figure 4.8: Momentum Corrections. Top, from left: $\Delta \mathrm{p}$ vs $p_{\text {measured }}, \Delta \lambda$ vs $p_{\text {measured }}$ and $\Delta \phi$ vs $p_{\text {measured }}$ distributions for the proton, for bin (Sector $1,26^{\circ}<\theta<48^{\circ}$, $\left.90^{\circ}<\phi<120^{\circ}\right)$. Bottom, from left: Gaussian means extracted from the above distributions fitted with a polynomial fit used to correct $\mathrm{p}$. 


\subsubsection{Time-of-flight of neutral particles correction}

Since one of the reactions of interest is $\gamma n(p) \rightarrow \pi^{+} \pi^{-} n(p)$, it is of primary importance for this analysis to correctly identify neutrons and determine their energy. Neutral particles were detected in CLAS as clusters in the electromagnetic calorimeter (EC, in the following) not associated with any reconstructed charged track from the drift chambers.

As we saw in section 2.5.5, the electromagnetic calorimeter was made up by six triangular sectors composed of alternating lead and scintillator strips that run parallel to the three $\mathrm{U}, \mathrm{V}$ and $\mathrm{W}$ axes of the triangle.

The reconstruction software recognized a cluster by identifying a collection of strips in each of the three views. The software required the PMT response to be above a threshold and the strips to be contiguous.

The centroid and root mean square (RMS) for each group was then calculated and, during the cooking of the data (see section 4.2), their values were registered in the ECHB bank along with the associated hit time for the event, as measured from the electromagnetic calorimeter. The hit time, or time-of-flight, is defined as the time from the event start time to the first PMT signal over threshold, simultaneously seen in all three views.

The ECHB bank also includes two flags (MatchID1 and MatchID2) which contain the information about whether the centroid has been reconstructed using only the outer stack of the EC (consisting of 8 layers of 3 views of scintillator) or both the inner stack (consisting of 5 layers of 3 views of scintillator) and the outer stack. The reconstructed cluster position, cannot provide the information about the exact location of the neutron interaction in the calorimeter, therefore the hit is assumed to be on the EC face (towards the target) of whichever of the two stacks the hit is contained in.

The information about the particle's vertex in this analysis is contained in the MVRT bank, which uses a multi-track-vertex fitting routine, giving a very accurate vertex for multiple charged particles that all come from the same vertex. During the cooking, the vertex of the fastest particle in the event (a pion in this analysis) is attributed also to the neutrals. Since in the reactions of our interest the neutron is coming directly from the primary vertex, this information is already accurate.

Using the reaction vertex coordinates $\left(x_{v}, y_{v}, z_{v}\right)$ and the EC cluster position $\left(x_{E C}, y_{E C}, z_{E C}\right)$, the directional components in terms of the polar and azimuthal angles $\theta$ and $\phi$ are calculated. The path length of the neutron is determined from the cluster position and the vertex position in the target. Using the EC time-of-flight, the momentum is then calculated. Neutral particles are tagged as neutrons if they have $\beta<0.9$, being $\beta$ calculated as:

$$
\beta=\frac{L_{\text {neutral }}}{c\left(T o F_{E C}-T_{v}\right)}=\frac{\sqrt{\left(x_{E C}-x_{v}\right)^{2}+\left(y_{E C}-y_{v}\right)^{2}+\left(z_{E C}-z_{v}\right)^{2}}}{c\left(T O F_{E C}-T_{v}\right)}
$$


where $T O F_{E C}$ is the neutron time time of flight, $T_{v}$ is the time at which the event occurs and $L_{\text {neutral }}$ is the neutral path length. The distribution of the values of $\beta$ for the neutrons are shown in Figure 4.9. To take into account a possible common offset of the time of flight measurements, the following procedure has been applied. The neutron time-of-flight registered in the ECHB bank is compared to the value $T_{\text {exp }}$, calculated as:

$$
T_{\text {exp }}=\frac{L_{\text {neutral }}}{c \beta_{\text {missing }}}+T_{\text {propagation }}+T_{\gamma}
$$

where the $L_{\text {neutral }}$ is the corrected neutron path, calculated as described before, $\beta_{\text {missing }}$ is the particle velocity $\left(\beta=p_{\text {exp }} / E_{\text {exp }}\right)$ calculated using the missing momentum and energy of the neutron from the reaction $\gamma d \rightarrow \pi^{+} \pi^{-} p(n), T_{\text {propagation }}$ is the time it takes for the photon to propagate from the center of CLAS to the vertex and $T_{\gamma}$ is the arrival time of the photon at the center of CLAS. Three different distributions $T_{\exp }-T_{E C}$, have been calculated for each stack combination (inner only, outer only and both inner and outer), and a different correction is found for each case.

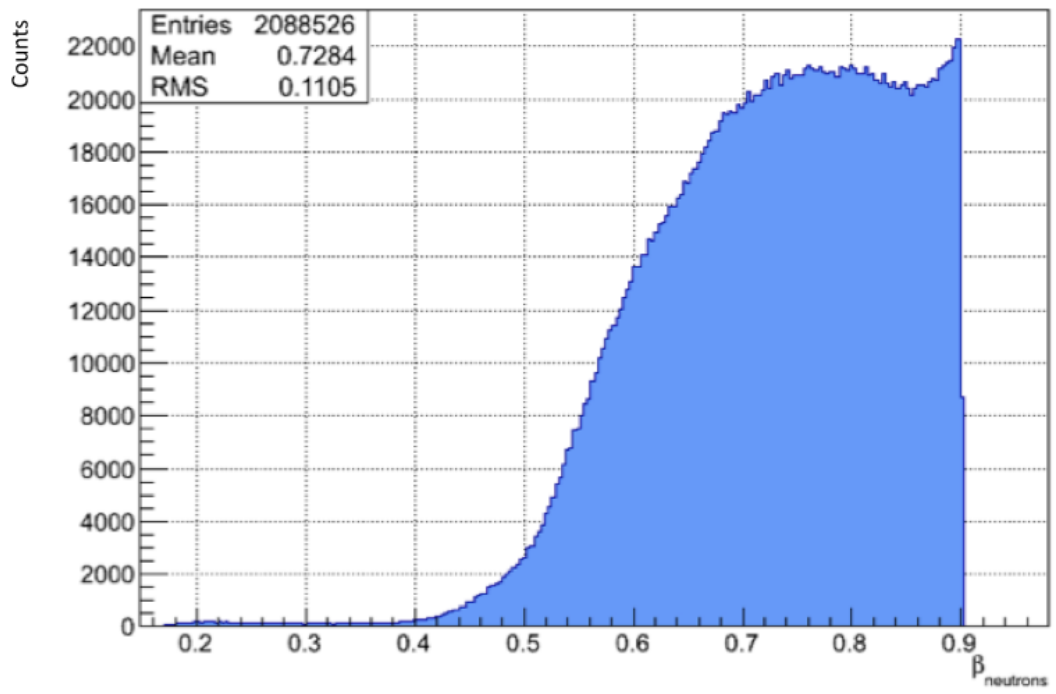

Figure 4.9: $\beta$ distribution for neutrons.

The calculated corrections for g14 were very small, due to the shortness of the HD-ice target, see section 3.2. 


\subsection{Neutron mis-identification}

One major problem that come out analyzing the data, was the presence of tracks erroneously attributed to neutrons. Comparing the polar angle of the detected neutron, $\theta_{\text {measured }}^{n}$, with the polar angle of the missing neutron, $\theta_{\text {expected }}^{n}$, from the reaction $\gamma n \rightarrow \pi^{+} \pi^{-}(n)$, we found that in many cases the direction of the detected neutron did not match the direction of the expected one. This can be seen in Figure 4.10, which shows the distribution of the difference $\Delta \theta^{n}=\theta_{\text {measured }}^{n}-\theta_{\text {expected }}^{n}$.
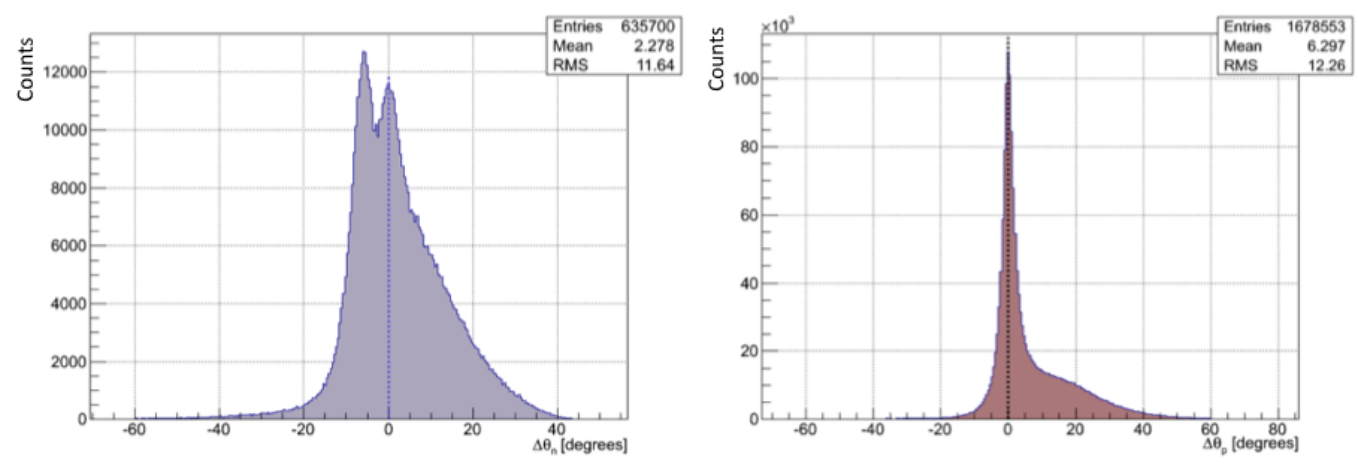

Figure 4.10: Left: distribution $\Delta \theta^{n}=\theta_{\text {measured }}^{n}-\theta_{\text {expected }}^{n}$ where $\theta_{\text {expected }}^{n}$ is calculated from the reaction $\gamma n \rightarrow \pi^{+} \pi^{-}(n)$; a double-peak structure is seen. The peak on the left side is due to protons which went through a low efficiency region of the drift chambers (see text for explanations). Right: distribution $\Delta \theta^{p}$ where $\theta_{\text {expected }}^{p}$ is calculated from the reaction $\gamma p \rightarrow \pi^{+} \pi^{-}(p)$; the double-peak structure is not seen here. The dataset used to make these plots was gold2 (see table 4.1).

The $\Delta \theta^{n}$ distribution shows a double-peak structure, where the peak centered around zero is associated with well-detected neutrons; our hypotheses was that the peak at $\Delta \theta^{n} \sim-0.8^{\circ}$ is due to protons. These protons, passing through a low efficiency region of the drift chambers subsystem, are bent by the magnetic field provided by the torus magnet, and hit the electromagnetic calorimeter at a different polar angle with respect to the neutrons, which follow straight trajectories.

Note that the double-peak it is not observed in the $\Delta \phi^{n}$ distribution, because the magnetic field provided by the the Torus does not change the azimuthal angle of charged particles, as seen in Figure 4.11.

The $\Delta \theta^{n}$ distribution was calculated using the gold2 dataset, which has been taken by setting a negative polarity for the torus. The peak in the $\Delta \theta^{n}$ distribution appears at $\Delta \theta^{n} \sim-0.8^{\circ}$, on the left side with respect to the peak, centered around zero. We checked our hypothesis plotting the same histogram of $\Delta \theta^{n}$ for events from 


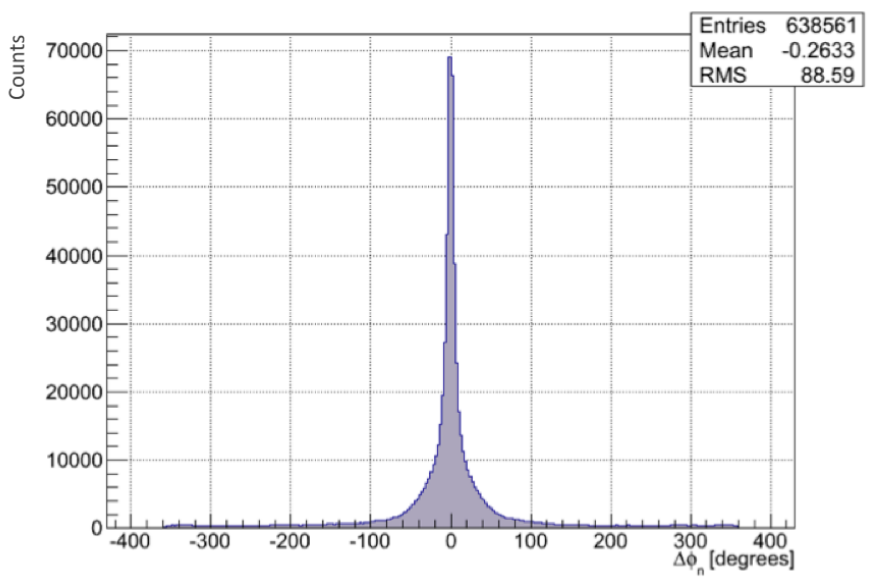

Figure 4.11: Distribution of $\Delta \phi^{n}$ for the reaction $\gamma n \rightarrow \pi^{+} \pi^{-}(n)$ : a double peak structure is not seen because the magnetic field is symmetric in the azimuthal angle.

datasets taken with positive currents for the torus (ex. silver 1 and silver 2).

The second peak due to the protons is expected to appear at the right of the peak centered around zero, as can bee seen in Figure 4.12.

In Figures 4.13 the angular distribution $(\theta$ vs $\phi)$ of the neutron, $\pi^{+}$and $\pi^{-}$are shown in the first row, and the corresponding plots of $\sin \theta \cos \phi$ vs $\sin \theta \sin \phi$ for the same particles are shown in the bottom row. The zones of low efficiencies in the Drift Chambers, correspond to holes in the angular distribution in the laboratory frame, of the $\pi^{+}$and the $\pi^{-}$mainly in sectors 3 and 5 . The same regions correspond to higher counts in the neutron plots.

In order to correctly identify neutrons, discarding events due to protons, we required that no hit is found in the scintillator counter subsystem in coincidence in the same timing window of a hit in the electromagnetic calorimeter; the events selected by this cut are shown in Figure 4.14.

In Figures 4.15 it is possible to see the effect of this cut on the 3-momentum conservation components, defined as follows:

$$
\begin{aligned}
& P_{x}^{r e a c}=-P_{x}^{\pi^{+}}-P_{x}^{\pi^{-}}-P_{x}^{n} \\
& P_{y}^{r e a c}=-P_{y}^{\pi^{+}}-P_{y}^{\pi^{-}}-P_{y}^{n} \\
& P_{z}^{r e a c}=P_{z}^{\gamma}-P_{z}^{\pi^{+}}-P_{z}^{\pi^{-}}-P_{z}^{n}
\end{aligned}
$$

After applying the described selection, the three distributions appear much narrower and correctly centered around zero. The tail on the left of the $P_{z}$ distribution is due to the fact that, at this point of the analysis, the kinematic is not closed yet. 

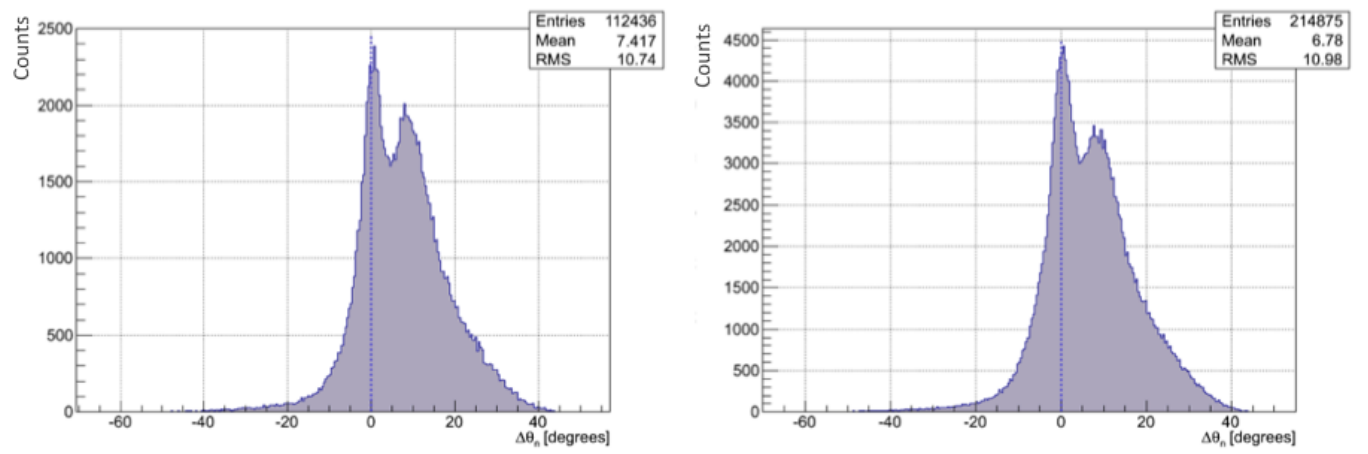

Figure 4.12: Left and right: distributions $\Delta \theta^{n}=\theta_{\text {measured }}^{n}-\theta_{\text {expected }}^{n}$ for the reaction $\gamma n \rightarrow \pi^{+} \pi^{-}(n)$ calculated using the datasets silver1 and silver2, respectively (see table 4.1), taken with a positive torus current. The peak not coming from wellidentified neutrons is seen on the right side of the distribution.
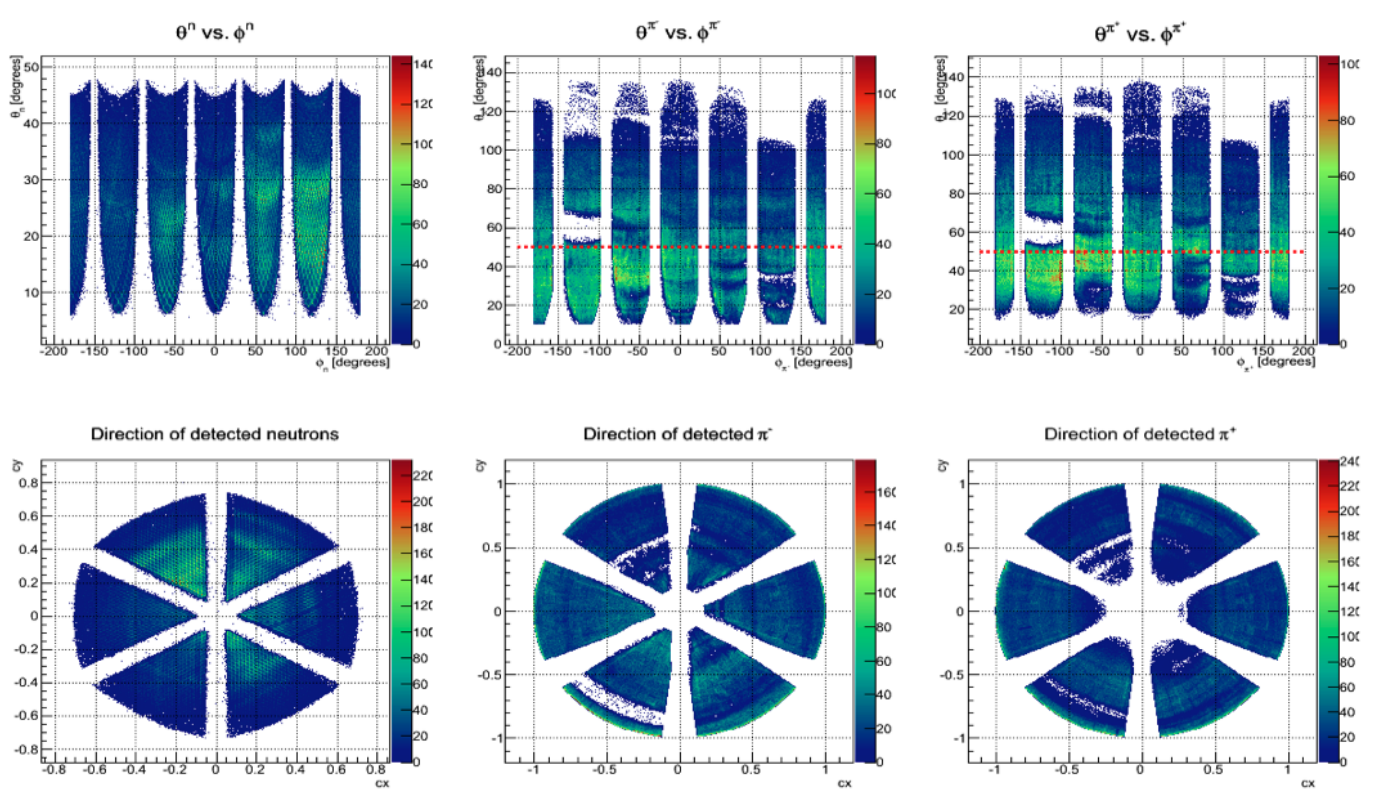

Figure 4.13: Angular distributions of neutrons and charged pions. The zones of low efficiencies in the Drift Chambers correspond to holes mainly in sectors 3 and 5. 


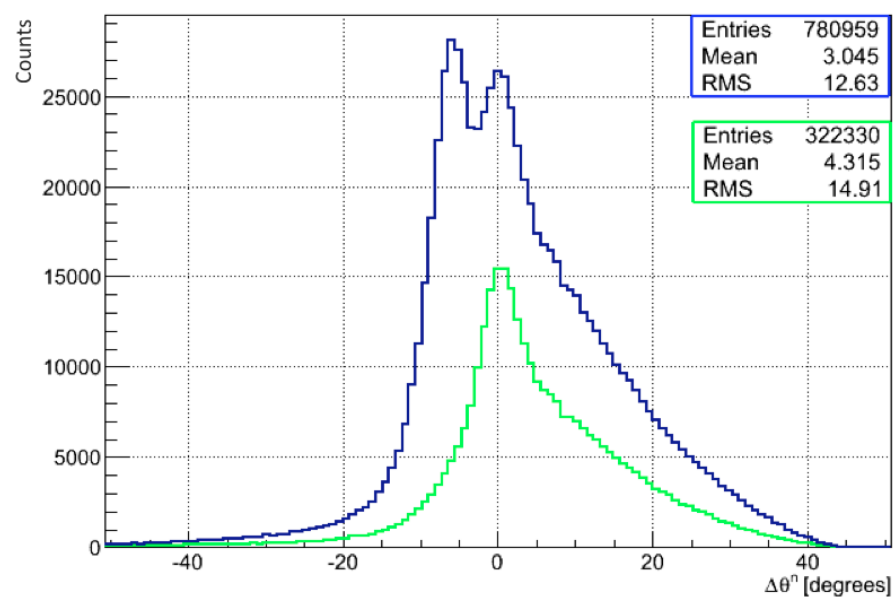

Figure 4.14: $\Delta \theta^{n}=\theta_{\text {measured }}^{n}-\theta_{\text {expected }}^{n}$ distribution before (blue histogram) and after (green histogram) imposing that there is no hit in the scintillator counter subsystem.
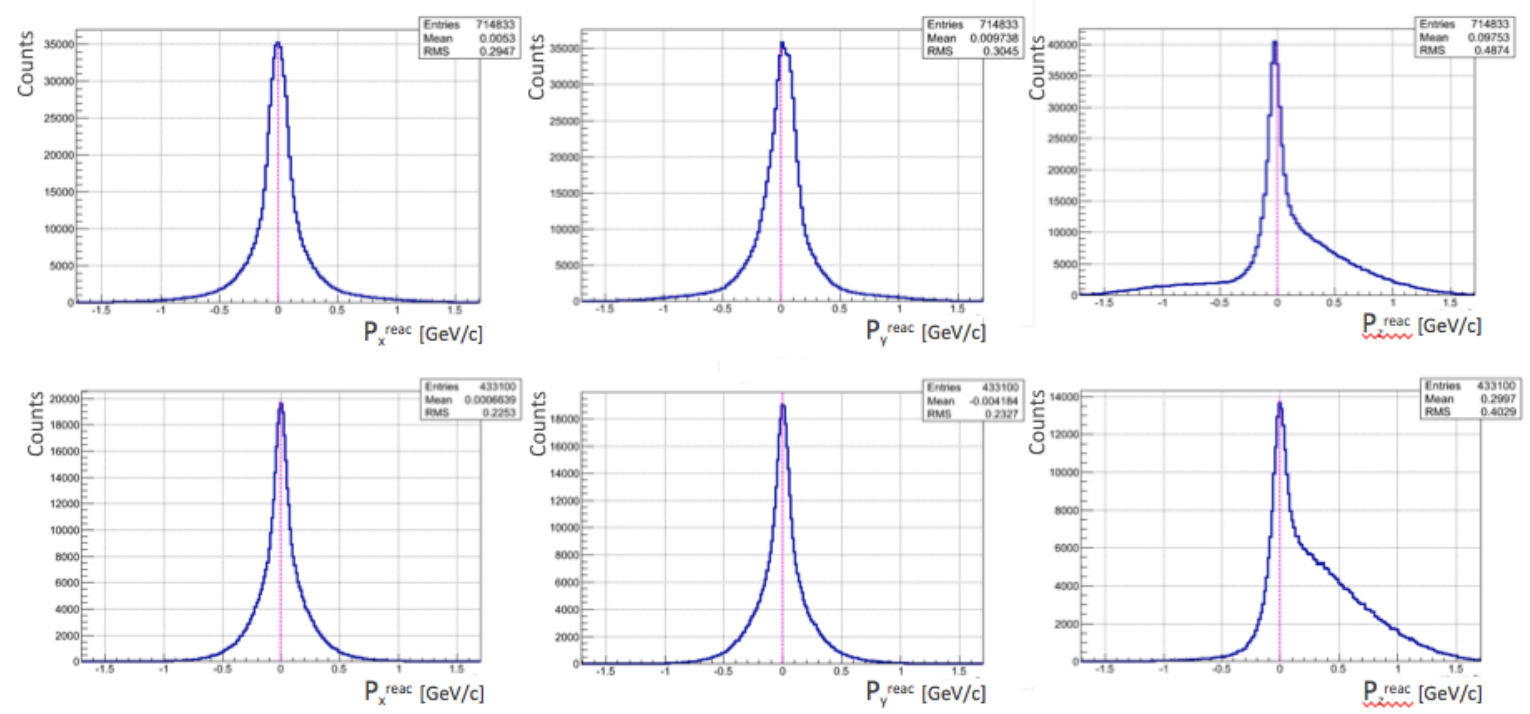

Figure 4.15: Tri-momentum conservation distributions, as defined in formulas 4.13, plotted before (top) and after (bottom) imposing the selection cut. 


\subsection{Event selection cuts}

The following sections describe the selection procedure applied to the skimmed and corrected events to identify those belonging to the reactions $\gamma \mathrm{p} \rightarrow \pi^{+} \pi^{-} \mathrm{p}$,

$\gamma \mathrm{p}(\mathrm{n}) \rightarrow \pi^{+} \pi^{-} \mathrm{p}(\mathrm{n})$ and $\gamma \mathrm{n}(\mathrm{p}) \rightarrow \pi^{+} \pi^{-} \mathrm{n}(\mathrm{p})$.

\subsection{1 z-vertex cut}

A cut was applied on the computed vertex of $\pi^{+}$and $\pi^{-}$for the reaction $\gamma n \rightarrow \pi^{+} \pi^{-} n$ and also on the proton for the reactions $\gamma p \rightarrow \pi^{+} \pi^{-} p$ and $\gamma p(n) \rightarrow \pi^{+} \pi^{-} p(n)$. The aim of this selection is to identify events produced in the HD cell volume and to exclude those originating from the cell windows and IBC Aluminum foils.

The vertex position of the particles for this analysis is taken from the MVRT banks, which is the result of a multi-track vertex fitting routine. This routine takes two tracks and finds the point midway on the shortest line segment between them, found with a least squares fit. If there is only one track, the interception between the track and the beamline coordinates is taken.

The cut imposed on the z-vertex coordinate is $-10 \mathrm{~cm}<$ z-vertex $<-6 \mathrm{~cm}$ (the g14 HD-ice target was located at $-7.5 \mathrm{~cm}$ from the CLAS centre); this interval was chosen after an accurate background subtraction study. The aluminum cooling wires placed inside the cell and the cell walls made of Kel-F (see section 3.2), contain the only unpolarizable nucleons of the target cell. In order to account for this background source, during the g14 running period few empty cell runs were acquired. In Figure 4.16 on the left, the z-vertex for all particles is shown; the red lines represent the cut imposed in this analysis. On the right the z-vertex distribution after imposing the cut is plotted (yellow histogram), superimposed to the vertex where no cut was applied (blue histogram). Figure 4.17 shows the z-vertex distribution for the empty target (green histogram) superimposed to the z-vertex distribution for the full target (red histogram); the z-vertex distribution for the full target subtracted for the scaled z-vertex distribution for the empty target is also shown (blue grid). The scaling factor was obtained from the ratio between the areas of the z-vertex histogram for the full target and the z-vertex histogram for the empty target.

\subsubsection{Incident Photon identification}

Another important step in the analysis, consists in the identification of the exact photon corresponding to the physics event. Since an event has multiple photons registered int the tagger bank, the actual photon is identified as the one whose time is closest to the event vertex time, according to the following formula:

$$
\Delta T=T_{\gamma}-T_{v}
$$



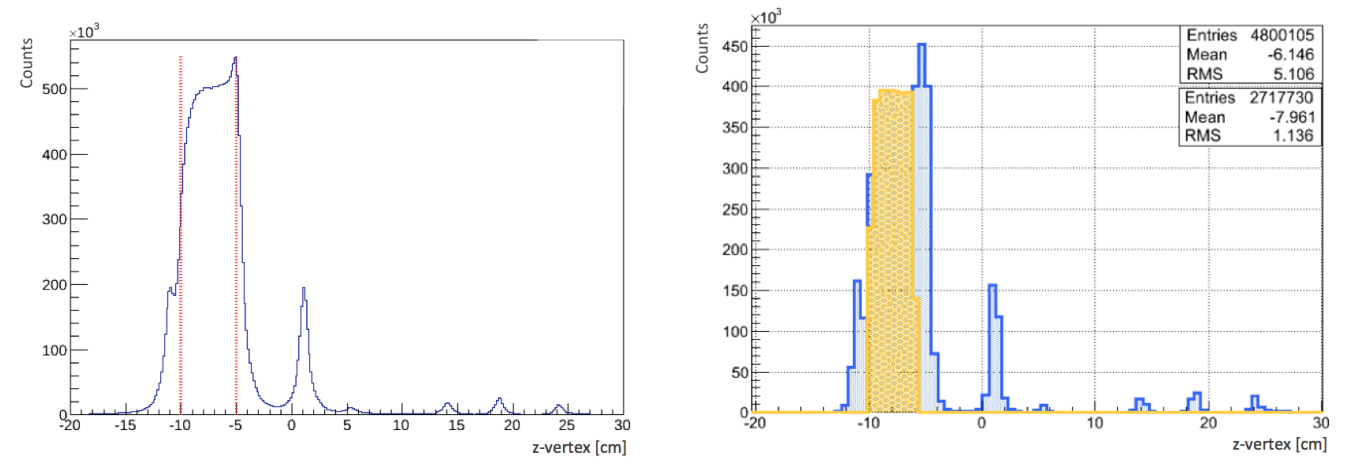

Figure 4.16: Left: z-vertex of all particles: the two vertical red lines represent the selection applied. Right: the yellow histogram is the z-vertex after the applied cut $-10 \mathrm{~cm}<\mathrm{z}$-vertex $<-6 \mathrm{~cm}$, the blue one is before the cut.

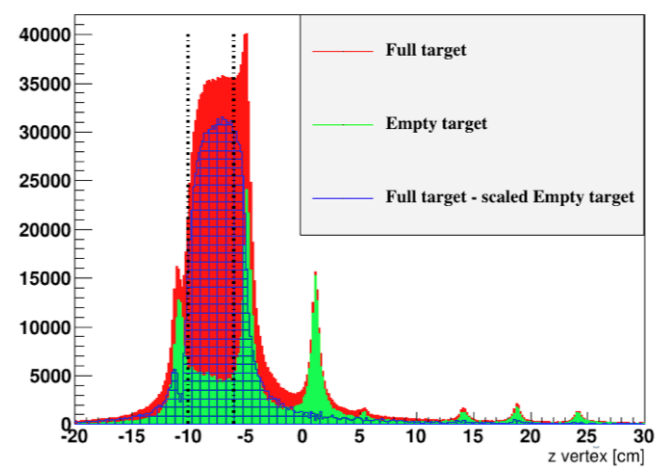

Figure 4.17: The zvertex for the full target (red histogram); superimposed are the empty target (green histogram) and the full target ? empty target scaled for the scaling factor (blue grid histogram) 
where $T_{v}$ is the time of the event vertex and is calculated from the charged hadron tracks as:

$$
T_{v}=T_{T o F}-\frac{L_{T o F}}{\beta c}
$$

where $T_{T o F}$ is the time at which the hadron was detected by the Time of Flight subsystem, $L_{T o F}$ is the calculated distance from the event vertex to the hit in the scintillator paddle, $\mathrm{c}$ is the speed of the light and $\beta$ is the hadron velocity calculated from the momentum, as measured in the Drift Chambers and assuming the nominal PDG mass. $T_{\gamma}$ is the arrival time of the photon at the event vertex, calculated as:

$$
T_{\gamma}=T_{\text {center }}+T_{\text {prop }}
$$

where $T_{\text {center }}$ is the time at which the photon arrives at the target centre, calculated from the tagger time information taking into account the electron path to the radiator and the photon path to the target cell. $T_{\text {prop }}$ is the photon propagation time from the nominal centre of the target to the interaction vertex. This latter may be calculated as:

$$
T_{\text {prop }}=\frac{z_{\text {vertex }}^{h}-z_{\text {target }}}{c}
$$

where $z_{\text {vertex }}^{h}$ is the z-vertex coordinate of the hadron $\left(\pi^{+}, \pi^{-}\right.$and $\mathrm{p}$, in this analysis) measured with respect to the centre of CLAS, $z_{\text {target }}$ is the exact target position (moved $-7.5 \mathrm{~cm}$ upstream of the CLAS detector in g14 experiment) and $\mathrm{c}$ is the speed of the light.

In order to define the selection cut, we studied the $\Delta T$ distributions, as expressed in formula 4.14, as a function of the final state charged particles momentum, separately for $\pi^{+}, \pi^{-}$and the proton, as shown in Figures 4.18. A $60 \mathrm{MeV} / \mathrm{C}$ momentum bin was used. For each momentum bin the $\Delta \mathrm{T}$ distribution was fitted with a Gaussian curve. The means and sigmas obtained from the gaussian fit, were, in turn, fitted by a third-order polynomial as a function of the particles momentum for values greater than $0.2 \mathrm{GeV}$, in the case of pions, and greater than $0.3 \mathrm{GeV}$, in the case of protons. A zeroth-order polynomial was used for lower momentum regions.

\subsubsection{Removing accidentals}

In order to remove accidentals, a cut is imposed on two variables from GPID banks, named NGRF and TAGRID. The NGRF variables indicates how many candidates photons the reconstruction code found, which passed the timing cut to find the incident photon. The TAGRID is an index which indicates the location of the photon related to a particle in the TAGR bank. In this analysis is required that, for all charged particles in the final state, NGRF must be equal to one; this means that for every particle in the final state there must be only one photon found which meets 

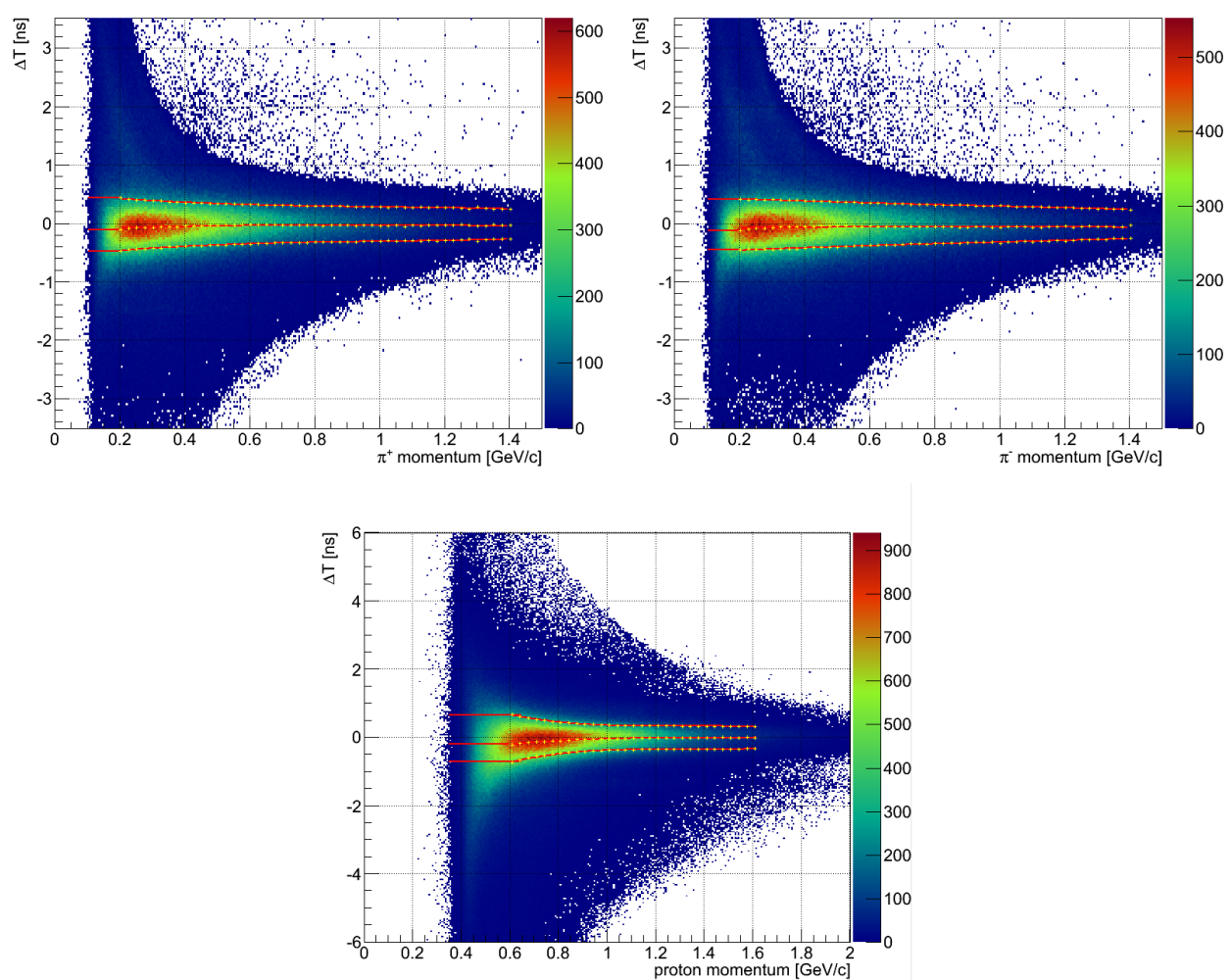

Figure 4.18: Upper Figure: Distribution $\Delta T=T_{\pi^{+}}-T_{\gamma}$ between all the candidate photons and the $\pi^{+}$as a function of the $\pi^{+}$momentum. Middle Figure: $\Delta T=T_{\pi^{-}}-$ $T_{\gamma}$ between all the candidate photons and the $\pi^{-}$as a function of the $\pi^{-}$momentum. Lower Figure: $\Delta T=T_{p}-T_{\gamma}$ between all the candidate photons and the proton as a function of the proton momentum. The yellow points are the means and the sigmas resulting from the gaussian fit as a function of the particles momentum; the red lines are the result of the polynomial fit on these points. The final cuts were chosen at $\pm 3 \sigma$. 
the timing requirements. As for the index TAGRID, in this work it is required that its values for all final state particles are the same; this means that the reconstruction code found the same code for all the particles in the final states. These cuts, along with the previous cut imposed to identify correctly the initial photon, ensures that the initial state is well-defined.

\subsubsection{Missing mass cut}

In order to identify the correct final state of the reactions of our interest, we applied a cut on the Missing Mass distribution $\operatorname{MM}\left(\pi^{+} \pi^{-}\right)$, to make sure that only the three particles $\pi^{+} \pi^{-} \mathrm{p}$ and $\pi^{+} \pi^{-} \mathrm{n}$ were produced. The distributions for proton and neutron targets are shown in Figure 4.19. The obtained distributions are fitted using a the total function (red curve) composed by a Breit-Wigner function (blue curve), a single gaussian (black curve) and a polynomial function (green curve).
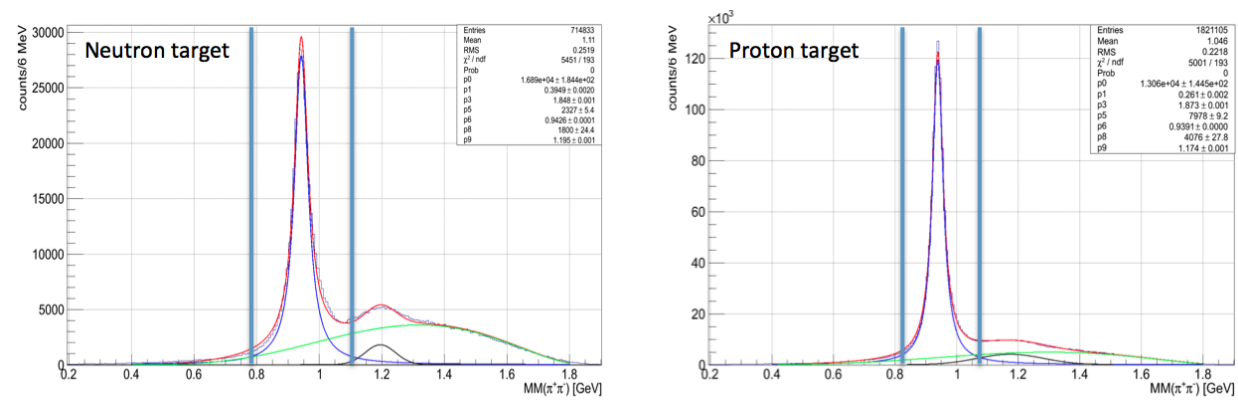

Figure 4.19: Left hand side: fit of the Missing Mass distribution $\operatorname{MM}\left(\pi^{+} \pi^{-}\right)$from a neutron target; right hand side: fit of the Missing Mass distribution $\operatorname{MM}\left(\pi^{+} \pi^{-}\right)$ from a proton target. The distributions are fitted with a function (red curve) which is the sum of a Breit-Wigner (blue-curve), a gaussian (black curve) and an exponential (green curve).

Good events are required to fall in a $200 \mathrm{MeV}(320 \mathrm{MeV})$ wide window around the nominal nucleon mass for the proton (neutron), respectively. These intervals correspond to approximately the $85 \%$ of the Breit-Wigner probably content for both target nucleons.

\subsection{5 $\Delta \beta$ cut}

In order to improve the identification of the charged particles in the final state $\left(\pi^{+}\right.$, $\pi^{-}$and the proton), a cut is imposed on the difference $\Delta \beta$, between $\beta$ measured from TOF, $\beta_{\text {ToF }}$, and $\beta$ calculated from momentum, $\beta_{p}$ : 


$$
\begin{gathered}
\beta_{T o F}=\frac{p a t h_{D C}}{c \cdot T_{t o f}} \\
\beta_{p}=\frac{p}{\sqrt{p^{2}+m_{P D G}^{2}}} \\
\Delta \beta=\beta_{T o F}-\beta_{p}
\end{gathered}
$$

where path $_{D C}$ is the charged pions or proton path from the interaction vertex to the scintillator counters tracked by the drift chambers, $T_{T o F}$ is the time measured in the scintillator counters, $\mathrm{p}$ is the pions or proton momentum and $m_{P D G}$ is the pions or proton PDG mass. The $\Delta \beta$ distributions as a function of the particles momentum are shown in Figures 4.20. The events were selected within a cut of $-0.05<\Delta \beta<0.05$ for the two charged pions and of $-0.02<\Delta \beta<0.02$ for the proton, as indicated by the straight red lines. 

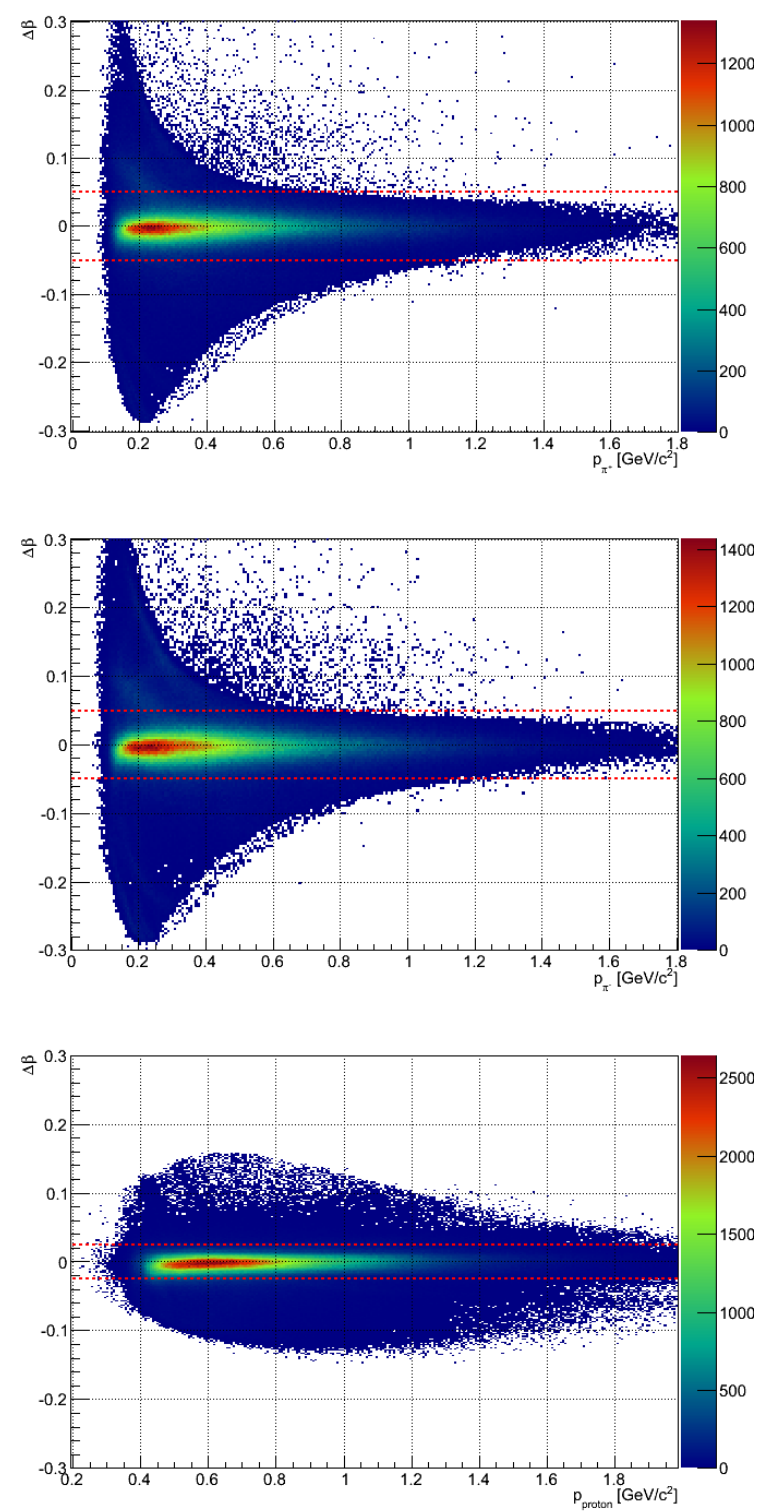

Figure 4.20: Loose cuts on the distributions $\Delta \beta$ vs particles momentum for $\pi^{+} \pi^{-}$ and proton; the selection is represented by the two red lines $(-0.05<\Delta \beta<0.05$ for the charged pions and $-0.02<\Delta \beta<0.02$ for the proton). 


\subsection{Final $\pi^{+} \pi^{-} p$ and $\pi^{+} \pi^{-} n$ sample}

In order to see the effect of all the selection cuts imposed, the distributions of $\beta_{\pi^{+}}$, $\beta_{\pi^{-}}$and $\beta_{\text {proton }}$ versus their respective particle momentum are shown in Figures 4.21 , before and after selection cuts. As can be seen, the applied cuts permitted to clean up the final sample very well. Figures 4.22 show monitoring plots of the event selection quality. The missing momentum of the reaction defined as:

$$
P_{\text {missing }}=\sqrt{\left(P_{x}^{\text {missing }}\right)^{2}+\left(P_{y}^{\text {missing }}\right)^{2}+\left(P_{z}^{\text {missing }}\right)^{2}}
$$

where

$$
\begin{aligned}
& P_{x}^{\text {missing }}=-P_{x}^{\pi^{+}}-P_{x}^{\pi^{-}}-P_{x}^{n} \\
& P_{y}^{\text {missing }}=-P_{y}^{\pi^{+}}-P_{y}^{\pi^{-}}-P_{y}^{n} \\
& P_{z}^{\text {missing }}=P_{z}^{\gamma}-P_{z}^{\pi^{+}}-P_{z}^{\pi^{-}}-P_{z}^{n}
\end{aligned}
$$

is shown in the upper part of the figures, for the proton and the neutron target events, before and after all selection cuts. Final histograms are compatible with the nucleon Fermi momentum distributions. The lower plots show the difference between the missing mass calculated from the incoming photon and the detected nucleon:

$$
M M(N)=\left(\tilde{P}_{\gamma}+\tilde{P}_{T}-\tilde{P}_{N}\right)^{2}
$$

and the invariant mass $I M\left(\pi^{+} \pi^{-}\right)$of the detected pions. As can be seen, the distributions differences $\mathrm{MM}(\mathrm{N})-I M\left(\pi^{+} \pi^{-}\right)$appear very narrow and well-centered around zero after all the cuts imposed in the analysis to select the final states $\pi^{+} \pi^{-} p$ and $\pi^{+} \pi^{-} n$. 

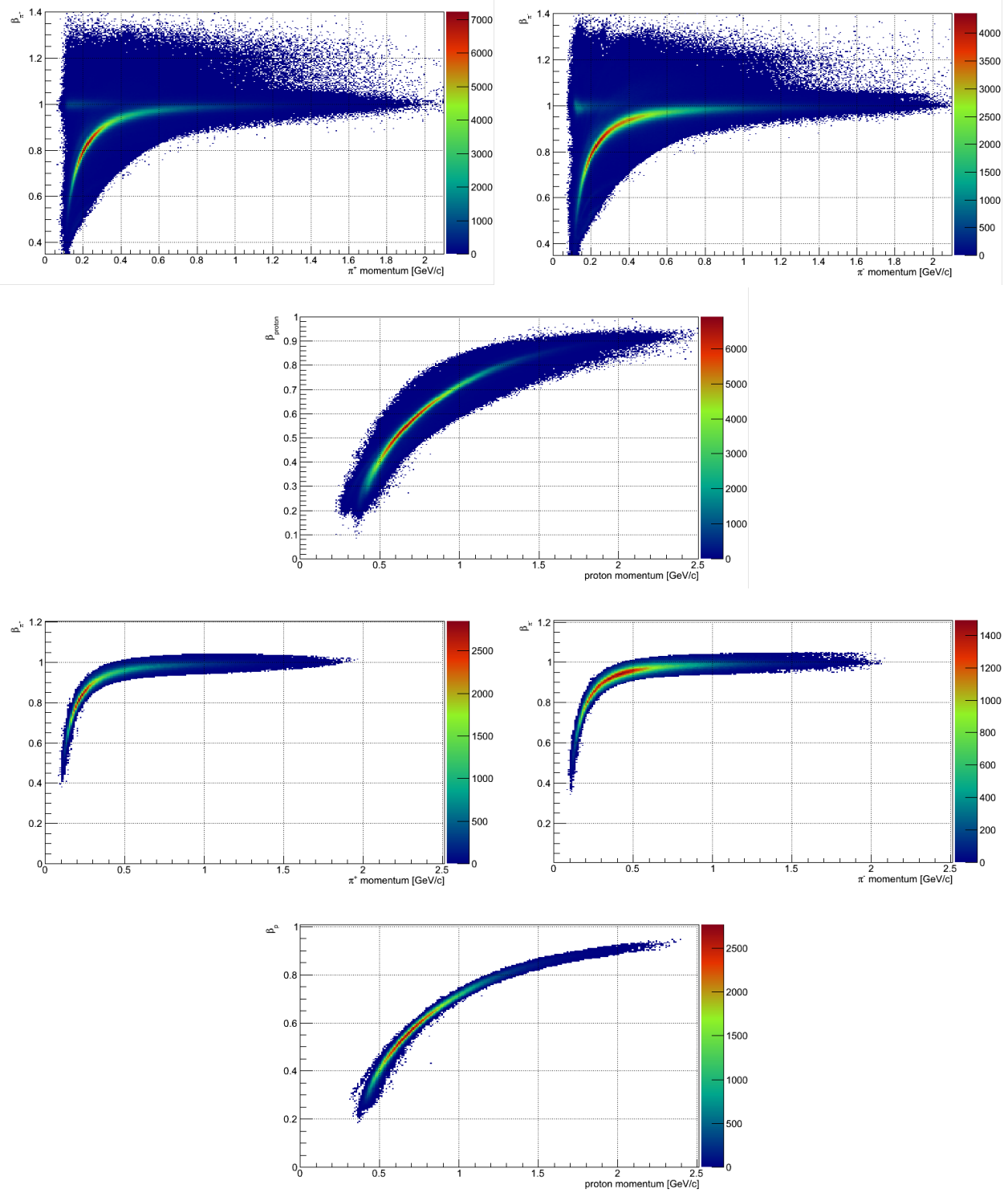

Figure 4.21: The distribution of $\beta_{\pi^{+}}, \beta_{\pi^{-}}$and $\beta_{\text {proton }}$ versus their respective particles momenta before (top) and after (bottom) all selection cuts applied. 

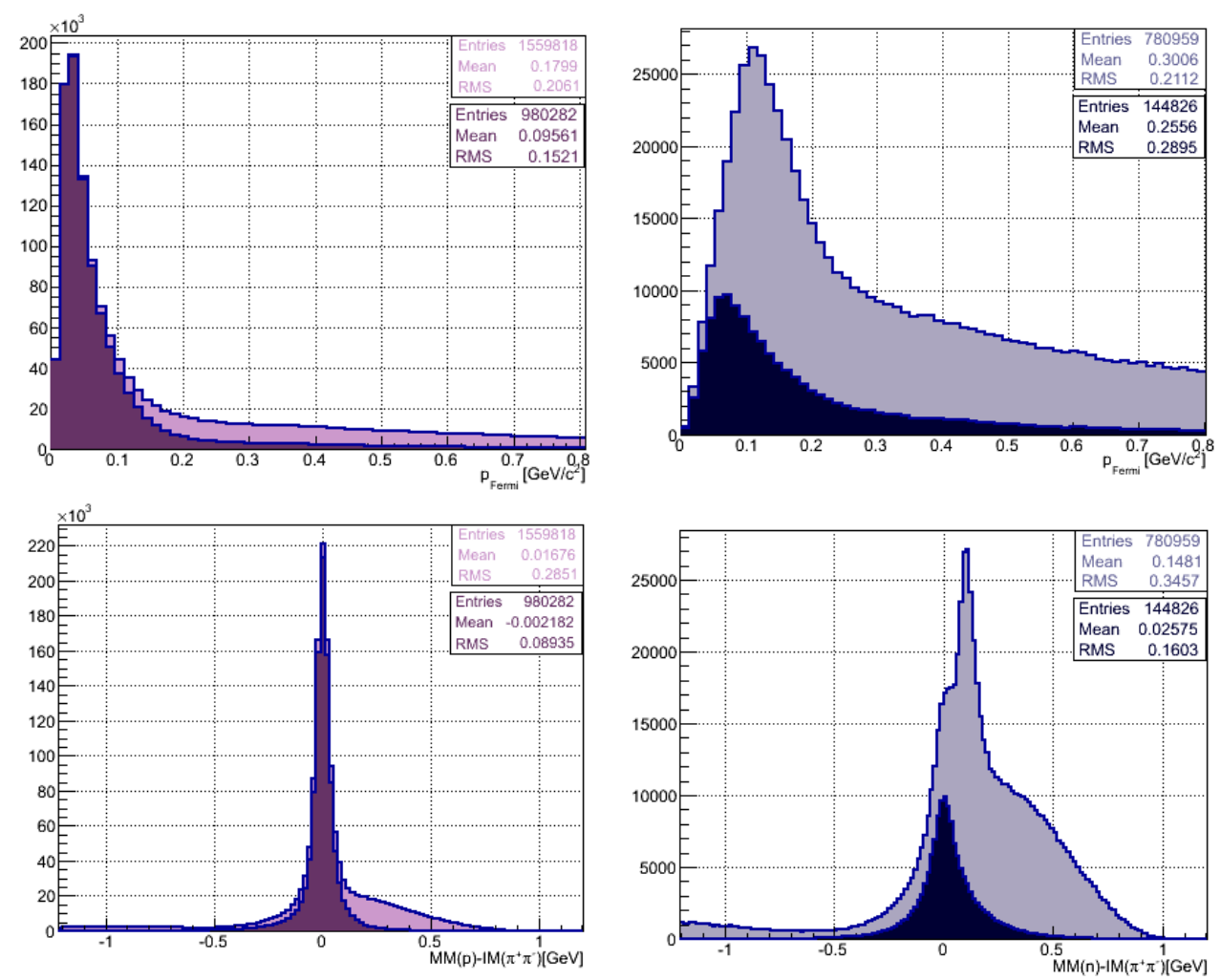

Figure 4.22: Left top and bottom: monitoring plots showing the Fermi momentum and the distribution difference $\operatorname{MM}(\mathrm{p})-I M\left(\pi^{+} \pi^{-}\right)$for the reaction $\gamma p \rightarrow \pi^{+} \pi^{-} p$. The distributions are shown before (pink histograms) and after imposing the selection cuts (dark pink histograms). Right top and bottom: same thing for the reaction $\gamma n \rightarrow \pi^{+} \pi^{-} n$; the distributions before imposing the cut are Violet; superimposed are the distributions after the imposition of the cuts, in black 


\section{Chapter 5}

\section{Extraction of polarization observables}

In this chapter we present the results of the polarization observables $I^{\odot}$ for the reactions $\gamma p \rightarrow \pi^{+} \pi^{-} p$ and $\gamma n \rightarrow \pi^{+} \pi^{-} n$ and $P_{z}^{\odot}$, for the reaction $\gamma p \rightarrow \pi^{+} \pi^{-} p$. In the last part of the chapter the selection procedure to identify the reaction $\gamma p \rightarrow \rho^{0} p$ is presented and the measurement of the double polarization observable $\mathrm{E}$ will be shown. A detailed explanation of the method used to extract these polarization observables from the experimental data will be provided.

\subsection{Angles definition and bin choice}

Before proceeding with the extraction of the polarization observables we describe the kinematic of the reaction $\gamma N \rightarrow \pi^{+} \pi^{-} N$ shown in Figure 5.1. The grey shadowed plane represents the center-of-mass production plane, defined by the incident photon and the recoiling nucleon momenta; the white plane represents the decay plane formed by the two final state pions. The angle $\phi_{\pi^{+}}^{\text {hel }}\left(\right.$ or $\phi_{\pi^{+}}^{\prime}$ ) is the azimuthal angle of the $\pi^{+}$meson in the rest frame of the $\pi^{+} \pi^{-}$system, also known as "helicity frame". It defines the orientation of the decay plane containing the two pions with respect to the production plane. The azimuthal angle $\phi_{\pi^{+}}^{\prime}$ can be determined from the pion momentum in the laboratory frame applying two boost and a rotation: a first boost along the beam line axis into the overall center-of-mass frame; a rotation around the direction perpendicular to the reaction plane transforms the $\mathrm{z}$ axis, formally aligned with the beam line, into the direction $z^{\prime}$, opposite to the recoil nucleon momentum. Finally, a second boost along $z^{\prime}$ results in the rest frame where the two pions are emitted back to back.

In this work the data are binned in two variables: $E_{\gamma}$, which is the incident photon energy in the Laboratory frame, and the azimuthal angle $\phi_{\pi^{+}}^{\text {hel }} . E_{\gamma}$ is divided into 7 bins $200 \mathrm{MeV}$ wide in the interval $E_{\gamma} \in[0.9,2.5] \mathrm{GeV}, \phi_{\pi^{+}}^{\text {hel }}$ is divided into 16 


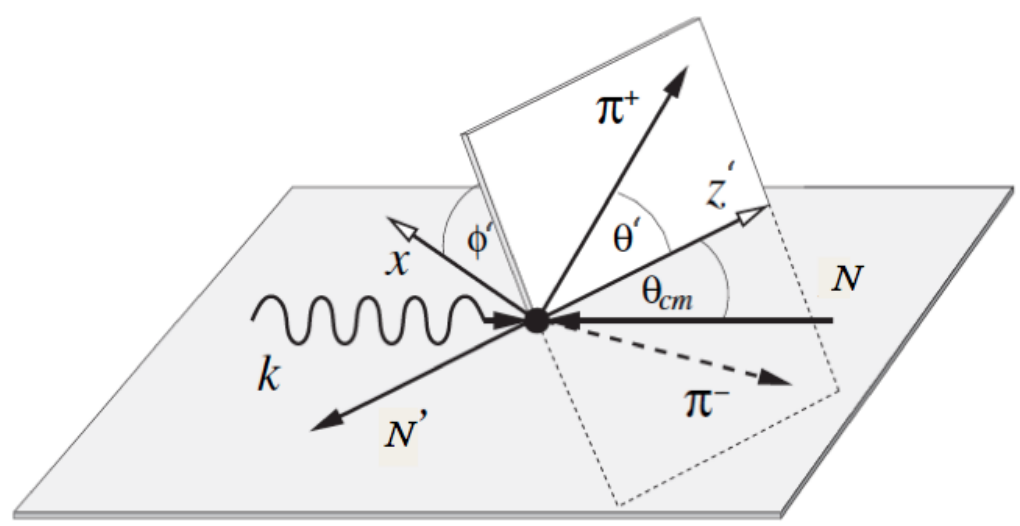

Figure 5.1: The grey shadowed plane represents the center-of-mass production plane formed by the incident photon and initial nucleon; the white plane represents the decay plane of the two final state pions. $\mathrm{K}$ denotes the initial photon and $\mathrm{N}$ the polarized target nucleon. $N^{\prime}$ is the final state nucleon, $\pi^{+}$and $\pi^{-}$are the two pions in the final state. $\theta_{c m}$ is the angle between the initial nucleon and the recoiling nucleon in the centre-of-mass system. $\phi_{\pi^{+}}^{\text {hel }}$ and $\theta_{\pi^{+}}^{\text {hel }}$ are the azimuthal and polar angle of the $\pi^{+}$in the helicity frame of the two pions.

bins of $\pi / 8$ width, covering a range $-\pi<\phi_{\pi^{+}}^{\text {hel }}<\pi$. We integrated over all the other independent kinematic variables.

\subsection{Observables for double pion photoproduction on the polarized protons and neutrons}

In the case of the $\gamma p \rightarrow \pi^{+} \pi^{-} p$ and $\gamma n \rightarrow \pi^{+} \pi^{-} n$ reactions, where the polarization of the recoiling nucleon is not measured, the differential cross section can be written as [21]:

$$
\begin{aligned}
& \frac{d \sigma}{d x_{i}}=\sigma_{0}(1+\vec{\Lambda} \cdot \overrightarrow{\mathbf{P}}) \\
& +\delta_{\odot}\left(\mathbf{I}^{\odot}+\vec{\Lambda} \cdot \overrightarrow{\mathbf{P}} \odot\right)+\delta_{l}\left[\sin 2 \beta\left(\mathbf{I}^{\mathbf{s}}+\vec{\Lambda}_{i} \cdot \overrightarrow{\mathbf{P}^{s}}\right)+\cos 2 \beta\left(\mathbf{I}^{\mathbf{c}}+\vec{\Lambda} \cdot \overrightarrow{\mathbf{P}^{c}}\right)\right]
\end{aligned}
$$

where $x_{i}$ are the kinematic variables of the reactions $\gamma N \rightarrow \pi^{+} \pi^{-} N$ and $\sigma_{0}$ is the unpolarized cross section. $\vec{\Lambda}$ is the polarization vector of the initial nucleon, $\delta_{\odot}$ and $\delta_{l}$ are the degree of circular and linear polarization of the photon beam, respectively. The polarization observables contained in 5.1 are a total of 15 and correspond to: 
- $\mathbf{I}^{\odot}, \mathbf{I}^{\mathbf{s}}$ and $\mathbf{I}^{\mathbf{c}}$ are observables which arise with a polarized photon beam. $\mathbf{I}^{\odot}$ is the beam asymmetry with an unpolarized target and a circularly polarized photon beam. $\mathbf{I}^{\mathbf{s}}$ and $\mathbf{I}^{\mathbf{c}}$ are obtained with a linearly polarized photon beams.

- $\overrightarrow{\mathbf{P}}$ describes the target asymmetry with a polarized target nucleon along the direction of $\Lambda$.

- $\overrightarrow{\mathbf{P}}$ and $\overrightarrow{\mathbf{P}}^{s, c}$ describe the double polarization observables measurable if both the target nucleon and the incoming photon are polarized, circularly or linearly, respectively.

In the present work the analyzed datasets were taken with circularly polarized photons and a longitudinal polarized target, in which case the cross section 5.1 reduces to:

$$
\frac{d \sigma}{d x_{i}}=\sigma_{0}\left\{\left(1+\Lambda_{z} \cdot \mathbf{P}_{\mathbf{z}}\right)+\delta_{\odot}\left(\mathbf{I}^{\odot}+\Lambda_{z} \cdot \mathbf{P}_{z}^{\odot}\right)\right\}
$$

The remaining measurable polarization observables are: $\mathbf{I}^{\odot}$ (beam-helicity asymmetry), $\mathbf{P}_{\mathbf{z}}$ (target asymmetry) and $\mathbf{P}_{\mathbf{z}}{ }^{\odot}$ (helicity difference).

The results of the polarization observable $\mathbf{I}^{\odot}$ for the reactions $\gamma N \rightarrow \pi^{+} \pi^{-} N$, from the g14 data, will be presented. The measurement of $\mathbf{I}^{\odot}$ from the double charged pion photoproduction off a proton target will be compared with the previous results published from CLAS g1c data [71] and CLAS g9 data [72] and with the result from the model of A.Fix [73].

The measurement of $\mathbf{I}^{\odot}$ from the double charged pion photoproduction off a neutron target are, instead, a first results available worldwide.

The measurement of $\mathbf{P}_{\mathbf{z}}{ }^{\odot}$ asymmetry from the double charged pion photoproduction off a proton target will be presented as well, and compared with the previous results from the CLAS g9 data [72] and with the model of A.Fix [73].

\subsection{Extraction of the Beam Helicity asymmetry $\left(I^{\odot}\right)$}

The differential cross section 5.2 may be written as:

$$
\frac{d \sigma}{d x_{i}}=\frac{N_{\text {events }}}{\epsilon \cdot F \cdot \rho \cdot \Delta x_{i}}=\frac{N_{\text {events }}}{\epsilon L \Delta x_{i}}
$$

being $N_{\text {events }}$ the number of events measured in the g14 experiment in the kinematical bin $\Delta x_{i}, \epsilon$ is the detection efficiency, $\mathrm{F}$ is the number of incoming photons, $\rho$ is the target area density and $L=F \cdot \rho$ is the integrated luminosity. Comparing equation 5.3 with the previous 5.2 we get:

$$
N_{\text {events }}=\sigma_{0}\left(\cdot L \cdot \Delta x_{i}\right)\left(1+\bar{\Lambda}_{z} \cdot \mathbf{P}_{\mathbf{z}}\right)+\bar{\delta}_{\odot}\left(\mathbf{I}^{\odot}+\bar{\Lambda}_{z} \cdot \mathbf{P}_{\mathbf{z}}^{\odot}\right)
$$


Since both the beam and the target are polarized in certain direction, in the following, we will use $\Rightarrow(\Leftarrow)$ to denote the target polarization parallel (antiparallel) to the beam axis and $\rightarrow(\leftarrow)$ to denote the beam polarization parallel (antiparallel) to the beam axis. In the following we will omit the terms $\epsilon$ and $\Delta \mathrm{x}_{i}$ since they will cancel out when considering the ratio of differences over sums of events in the same bin, to calculate the asymmetry. One can explicitly write down the equation 5.4 for the different combinations of target and beam polarization alignment:

$$
\begin{aligned}
& N_{\sigma(\rightarrow \Rightarrow)}=L(\rightarrow \Rightarrow)\left(1+\bar{\Lambda}_{z}(\Rightarrow) \mathbf{P}_{\mathbf{z}}+\bar{\delta}_{\odot}(\rightarrow)\left(\mathbf{I}^{\odot}+\bar{\Lambda}_{z}(\Rightarrow) \mathbf{P}_{\mathbf{z}}^{\odot}\right)\right. \\
& N_{\sigma(\leftarrow \Rightarrow)}=L(\leftarrow \Rightarrow)\left(1+\bar{\Lambda}_{z}(\Rightarrow) \mathbf{P}_{\mathbf{z}}-\bar{\delta}_{\odot}(\leftarrow)\left(\mathbf{I}^{\odot}+\bar{\Lambda}_{z}(\Rightarrow) \mathbf{P}_{\mathbf{z}} \odot\right)\right. \\
& N_{\sigma(\rightarrow \Leftarrow)}=L(\rightarrow \Leftarrow)\left(1-\bar{\Lambda}_{z}(\Leftarrow) \mathbf{P}_{\mathbf{z}}+\bar{\delta}_{\odot}(\rightarrow)\left(\mathbf{I}^{\odot}-\bar{\Lambda}_{z}(\Leftarrow) \mathbf{P}_{\mathbf{z}} \odot\right)\right. \\
& N_{\sigma(\leftarrow \Leftarrow)}=L(\leftarrow \Leftarrow)\left(1-\bar{\Lambda}_{z}(\Leftarrow) \mathbf{P}_{\mathbf{z}}-\bar{\delta}_{\odot}(\leftarrow)\left(\mathbf{I}^{\odot}-\bar{\Lambda}_{z}(\Leftarrow) \mathbf{P}_{\mathbf{z}} \odot\right)\right.
\end{aligned}
$$

In the case of the g14 experiment the polarization of the electron beam in each run period was flipped 960 times per second, while the target direction was kept fixed during each dataset. This means that for a single run period one has only events of the type $N(\rightarrow \Rightarrow)$ and $N(\leftarrow \Rightarrow)$ or $N(\rightarrow \Leftarrow)$ and $N(\leftarrow \Leftarrow)$. Therefore, in order to extract the polarization observable $I^{\odot}$ from the g14 data, it is necessary to combine different dataset taken with opposite target polarizations. We can assume that $L(\rightarrow)=L(\leftarrow)$ but $L(\Rightarrow)$ and $L(\Leftarrow)$ will differ for each run period; we will have to take into account the different luminosities when combining different periods.

In this work we combine the two datasets gold2 and silver5 (see table 4.1), for which the value of the luminosities ratio, the beam and target polarizations are:

- $\delta_{\odot}^{g 2}=83.4 \% \quad \delta_{\odot}^{s 5}=88.8 \%$

- $\Lambda(H)_{z}^{g 2}=27.6 \%, \Lambda(D)_{z}^{g 2}=26.9 \% \quad \Lambda(H)_{z}^{s 5}=-0.8 \%, \Lambda(D)_{z}^{s 5}=-6.0 \%$

- $\frac{L g 2}{L s 5}=0.36$

where the superscripts g2 and s5 are the short form for gold2 and silver5 run periods, respectively.

The $I^{\odot}$ asymmetry may be extracted from relations (5.5) as:

$$
\begin{gathered}
I^{\odot}=\frac{1}{\delta_{\odot}^{g 2}} \frac{\left[N(\rightarrow \Rightarrow)^{g 2}-N(\leftarrow \Rightarrow)^{g 2}\right]}{\left[N(\rightarrow \Rightarrow)^{g 2}+N(\leftarrow \Rightarrow)^{g 2}\right]+\frac{L^{g 2}}{L^{s 5}} \frac{\Lambda_{z}^{g 2}}{\Lambda_{z}^{s 5}}\left[N(\rightarrow \Leftarrow)^{s 5}+N(\leftarrow \Leftarrow)^{s 5}\right]}+ \\
+\frac{1}{\delta_{\odot}^{s 5}} \frac{L^{g 2}}{L^{s 5}} \frac{\Lambda_{z}^{g 2}}{\Lambda_{z}^{s 5}} \frac{\left[N(\rightarrow \Leftarrow)^{s 5}-N(\leftarrow \Leftarrow)^{s 5}\right]}{\left[N(\rightarrow \Rightarrow)^{g 2}+N(\leftarrow \Rightarrow)^{g 2}\right]+\frac{L^{g 2}}{L^{s 5}} \frac{\Lambda_{z}^{g 2}}{\Lambda_{z}^{s 5}} N\left[(\rightarrow \Leftarrow)^{s 5}+N(\leftarrow \Leftarrow)^{s 5}\right]}
\end{gathered}
$$


Figures 5.2 show the observable $I^{\odot}$ extracted from the analysis of the channel $\gamma p \rightarrow \pi^{+} \pi^{-} p$ from the g14 data. The expected odd symmetry for this variable is clearly observed in the data. This result is compared to the previous works of S. Strauch [71], who used the CLAS-g1c data [71] and S. Park [72], who used the CLAS-g9 data. The observable $I^{\odot}$ from the g14 experiment (blue dots) is in overall agreement with the previous works. The measurement of the observable $I^{\odot}$ is also compared to the results from the model of A. Fix [73] which are available for photon energies from threshold up $1.5 \mathrm{GeV}$. The calculation from A.Fix shows the expected odd asymmetry, however the agreement between the polarization observable extracted from the g14 data and the model is not very good.

Figures 5.3 show the observable $I^{\odot}$ extracted from the analysis of the channel $\gamma n \rightarrow \pi^{+} \pi^{-} n$. Currently, there are no models and, most of all no previous works with which compare this measurement to, since these are the first results for the extraction of the observable $I^{\odot}$ off a neutron target in the world. 

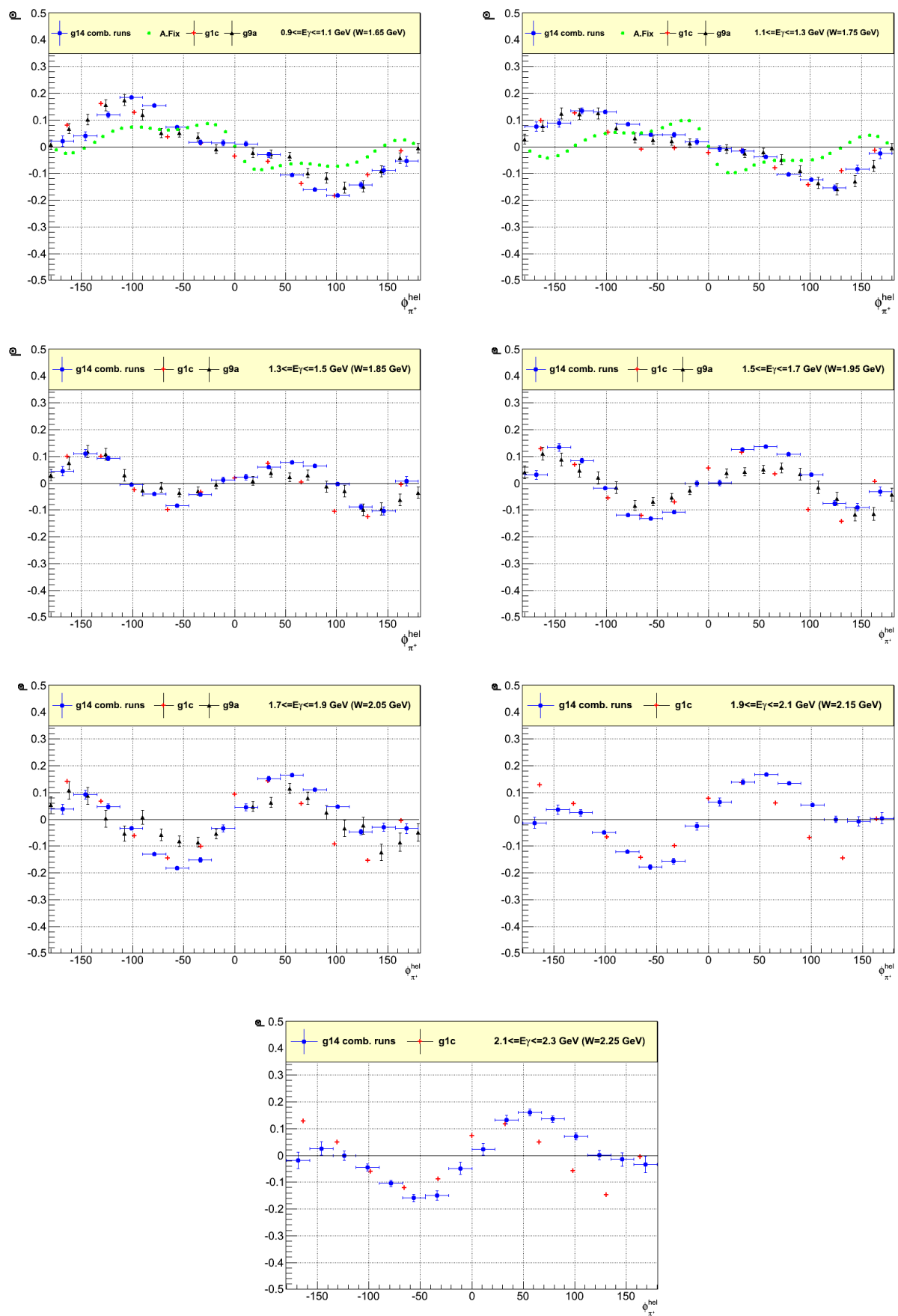

Figure 5.2: Measured beam-helicity asymmetry $I^{\odot}$ for the reaction $\gamma p \rightarrow \pi^{+} \pi^{-} p$, as a function of $\phi_{\pi^{+}}^{\text {hel }}$, in the Photon Energy Range $E_{\gamma} \in[0.9,2.3] \mathrm{GeV}$. Blue points are from the present work, black points from g9a analysis [72], red points from the g1c analysis [71]. Green points are theoretical calculation from A. Fix [73]. 

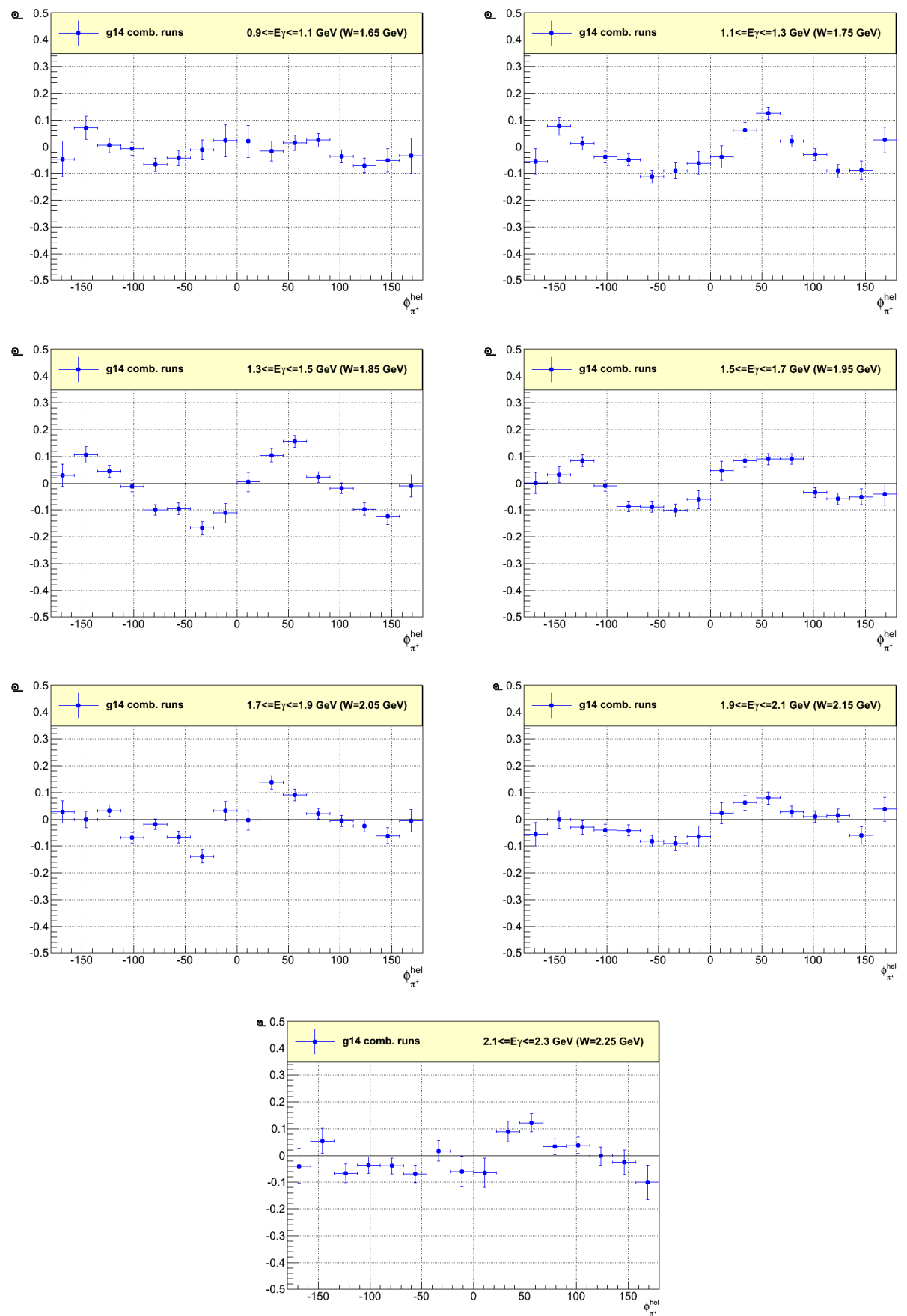

Figure 5.3: Measured beam-helicity asymmetry $I^{\odot}$ for the reaction $\gamma n \rightarrow \pi^{+} \pi^{-} n$, as a function of $\phi_{\pi^{+}}^{\text {hel }}$, in the Photon Energy Range $E_{\gamma} \in[0.9,2.3] \mathrm{GeV}$. 


\subsection{Extraction of the Helicity Difference $\left(P_{z}^{\odot}\right)$}

The extraction of the double polarization observable $\mathbf{P}_{\mathbf{z}}{ }^{\odot}$ requires data with a longitudinally polarized target and a circularly polarized beam. As in the previous case, it's necessary to combine datasets having opposite direction of the target polarization. The same considerations done before about the luminosities for different dataset apply. The final expression to extract the polarization observable $\mathbf{P}_{\mathbf{z}}{ }^{\odot}$ from the data is obtained solving the equations (5.5) as follows:

$$
\begin{aligned}
& P_{z}^{\odot}=\frac{1}{\delta_{\odot}^{g 2} \Lambda_{z}^{s 5}} \frac{\left[N(\rightarrow \Rightarrow)^{g 2}-N(\leftarrow \Rightarrow)^{g 2}\right]}{\left[N(\rightarrow \Rightarrow)^{g 2}+N(\leftarrow \Rightarrow)^{g 2}\right]+\frac{L^{g 2}}{L^{s 5}} \frac{\Lambda_{z}^{g 2}}{\Lambda_{z}^{s 5}}\left[N(\rightarrow \Leftarrow)^{s 5}+N(\leftarrow \Leftarrow)^{s 5}\right]}+ \\
& +\frac{1}{\delta_{\odot}^{s 5}} \frac{L^{g 2}}{L^{s 5}} \frac{1}{\Lambda_{z}^{s 5}} \frac{\left[N(\rightarrow \Leftarrow)^{s 5}-N(\leftarrow \Leftarrow)^{s 5}\right]}{\left[N(\rightarrow \Leftarrow)^{g 2}+N(\leftarrow \Leftarrow)^{g 2}\right]+\frac{L^{g 2}}{L^{s 5}} \frac{\Lambda_{z}^{g 2}}{\Lambda_{z}^{s 5}} N\left[(\rightarrow \Leftarrow)^{s 5}+N(\leftarrow \Leftarrow)^{s 5}\right]}
\end{aligned}
$$

where the notation has already been described in the previous section. Figures 5.4 show the measurement of the polarization observable $P_{z}^{\odot}$ for the reaction $\gamma p \rightarrow \pi^{+} \pi^{-} p$ from the g14 data; the measured observable shows the expected even symmetry. The result is compared with the model by A.Fix [73]; the overall agreement is good, in shape but not in amplitude. The comparison with the data from the g9 experiment [72] shows a good agreement. 

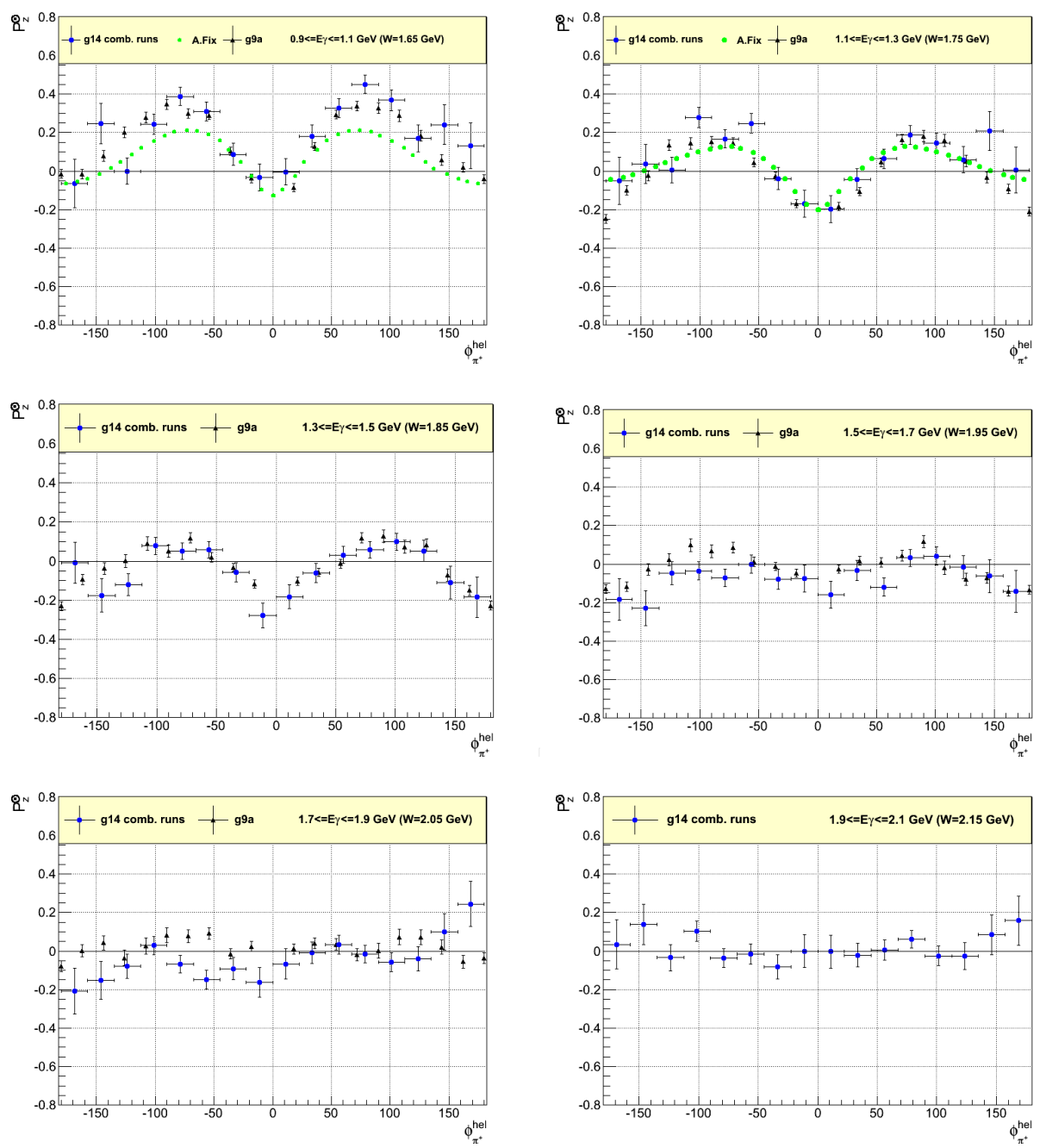

Figure 5.4: Measured helicity difference $P_{z}^{\odot}$ for the reaction $\gamma p \rightarrow \pi^{+} \pi^{-} p$, as a function of $\phi_{\pi^{+}}^{\text {hel }}$, in the Photon Energy Range $E_{\gamma} \in[0.9,2.1] \mathrm{GeV}$. Blue points are from the present work, black points from g9a analysis [72] (FROST), red points from the g1c analysis [71]. Green points are theoretical calculation from A. Fix [73]. 


\subsection{The reaction $\gamma \mathrm{p} \rightarrow \rho^{0} \mathrm{p}$}

\subsubsection{The $\rho$ vector meson}

The $\rho$ vector meson is a strongly interacting particle which exists in a triplet of isospin: $\rho^{0}, \rho^{+}$and $\rho^{-}$. It has a mass of $770 \mathrm{MeV}$ and a decay width of $\sim 145 \mathrm{MeV}$. A small mass difference between the $\rho^{+}$and the $\rho^{0}$ that can be attributed to the electromagnetic self-energy of the particle, as well as a small effect due to isospin breaking arising from the light quark masses; the current experimental limit is that this mass difference is less than $0.7 \mathrm{MeV}$. Like the other vector mesons, such as $\omega$ and $\phi$, the $\rho$ meson carries spin-parity quantum numbers $J^{P C}=1^{--}$and has the same quantum numbers of the photon. The differential cross sections for the $\rho$ photoproduction show an exponential fall-off at small value of the squared recoil momentum t, as shown in Figure 5.5. $\mathrm{t}$ is defined as:

$$
t=\left(\tilde{P}_{\gamma}-\tilde{P}_{\rho}\right)^{2}=\left(\tilde{P}_{N}-\tilde{P}_{N^{\prime}}\right)^{2}=m_{\rho}^{2}-2 E_{\gamma}\left(E_{\rho}-p_{\rho} \cos \theta_{\rho}\right)
$$

where $\tilde{P}_{\gamma}, \tilde{P}_{\rho}, \tilde{P}_{n}$ and $\tilde{P}^{\prime}$ are the four-vectors of the incident photon, the photoproduced $\rho$ vector meson, the neutron target and the neutron recoil in the final state, respectively; $E_{\gamma}$ is the energy of the incident photon and $E_{\rho}, p_{\rho}$ and $\theta_{\rho}$ are the energy, momentum and polar angle of the $\rho$ meson in the lab frame.

This trend implies that the process has more probability to happen at small values of $t$, or, equivalently at forward angles.

This is called diffractive behavior and it was explained in terms of the Vector Meson Dominance model (VDM), developed by Sakurai in 1960, according to which the interaction between the photon and the nucleon target is dominated by an inflight transformation of the photon into a vector meson which is scattered by the nucleon, as shown in Figure 5.6.

More recent versions of this model describe the diffractive behavior in terms of t-channel exchanges. The first term is the t-channel exchange of a particle having the same quantum numbers of the vacuum and it is called Pomeron; the exchange of the Pomeron occurs with parity $P=(-1)^{J}$, the so-called natural parity, being $\mathrm{J}$ the total angular momentum. In Figure 5.7 the Pomeron exchange contribution to the reaction amplitude is shown in the panel (a). The second term is the tchannel exchange of a pseudoscalar meson $\left(\pi^{0}\right.$ or $\left.\eta\right)$; the exchange occurs with parity $P=(-)^{J+1}$, the so-called unnatural parity. The contribution of the pseudoscalar meson exchange is shown in panel (b) of Figure 5.7. Contributions from s and $u$ exchange channels are shown in panels (c) and (d) of Figure 5.7, respectively, which include the contributions from baryon resonances.

In the previous chapter the procedure for obtaining a clean sample of events containing $1 \pi^{+}, 1 \pi^{-}$and 1 nucleon in the final state was fully explained. Our next goal is to identify the $\rho^{0}$ events and ultimately to disentangle them from the background, which in the case of a proton target is due to the following reactions: 

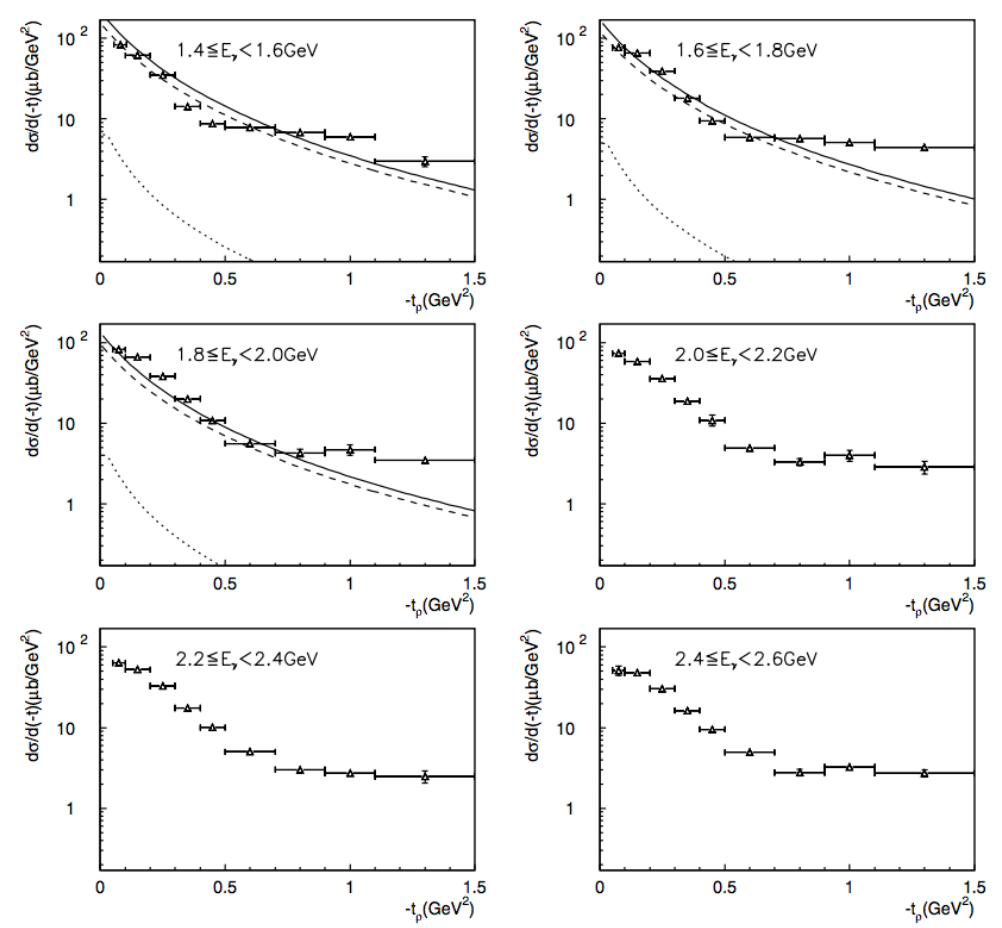

Figure 5.5: Differential cross-section of the reaction $\gamma \mathrm{p} \rightarrow \mathrm{p} \rho_{0}$ (Söding model extraction) from SAPHIR collaboration [68]. The curves are from [4]. Dashed lines: full model; dotted lines: $\pi$-exchange contribution; full lines: fit of this model to our data. 


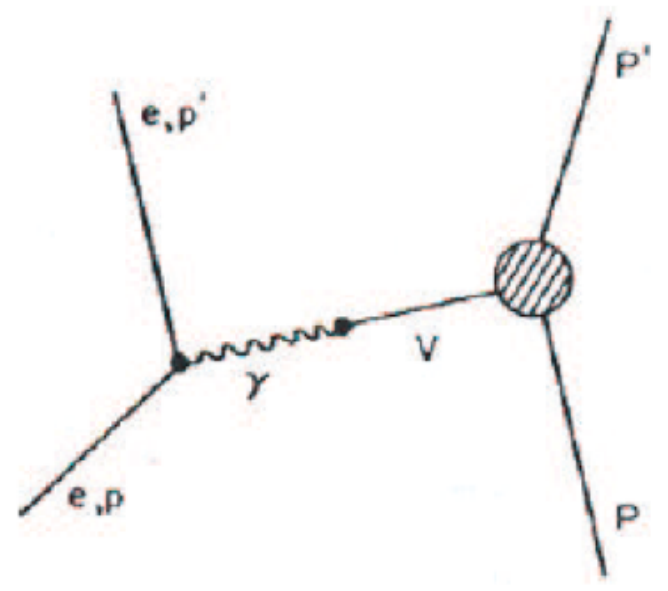

Figure 5.6: Vector Meson Dominance Model

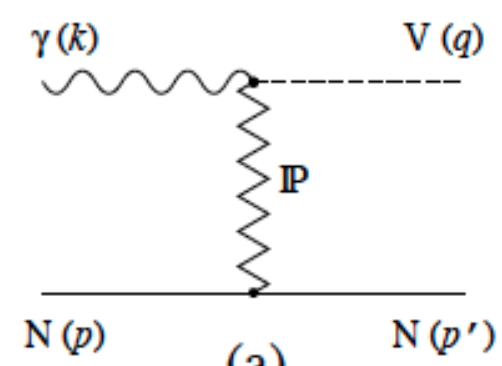

(a)

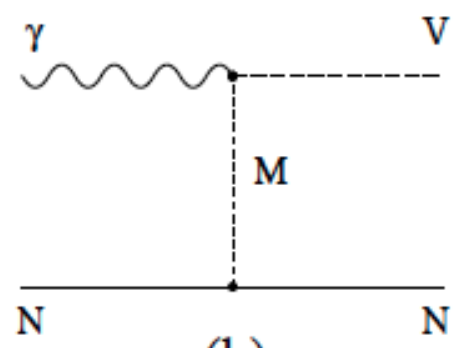

(b)

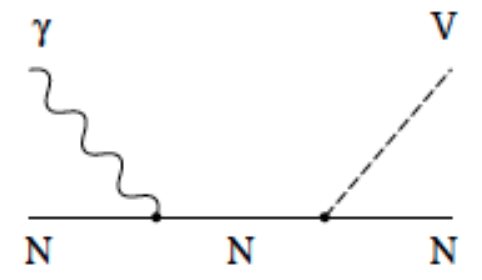

(c)

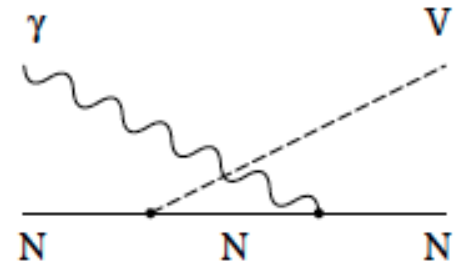

(d)

Figure 5.7: Diagrammatic rappresentation of $\rho$ photoproduction mechanism. (a,b) tchannel Pomeron and one-meson exchanges $\left(\mathrm{M}=f_{2}, \pi, \eta, \sigma\right)$, s- and $\mathrm{u}$-channel nucleon pole terms. 


\begin{tabular}{|l|l|l|l|l|l|l}
\hline & $I^{G}$ & $J^{P C}$ & Mass $(\mathrm{MeV})$ & Width $(\mathrm{MeV})$ & Decay mode & B.R. \\
\hline$\rho^{0}$ & $1^{+}$ & $1^{--}$ & $769.9+0.8$ & $151.2+1.2$ & $\rightarrow \pi^{+} \pi^{-}$ & $100 \%$ \\
\hline$\Delta^{++}$ & $\frac{3}{2}$ & $\frac{3}{2}^{+}$ & $1230.9+0.5$ & $112.2+1.5$ & $\rightarrow \mathrm{p} \pi^{+}$ & $>99 \%$ \\
\hline$\Delta^{0}$ & $\frac{3}{2}$ & $\frac{3}{2}^{+}$ & $1230.9+0.5$ & $112.2+1.5$ & $\rightarrow \mathrm{p} \pi^{-}$ & $>99 \%$ \\
\hline
\end{tabular}

Table 5.1: Properties of the $\rho$ and $\Delta^{\prime} s$ form PDG

- $\gamma \mathrm{p} \rightarrow \pi^{+} \pi^{-} \mathrm{p}$-non resonant photoproduction of $\pi^{+} \pi^{-}$a pair of

- $\gamma \mathrm{p} \rightarrow \pi^{-} \Delta^{++} \rightarrow \pi^{-} \pi^{+} \mathrm{p}$

- $\gamma \mathrm{p} \rightarrow \pi^{+} \Delta^{0} \rightarrow \pi^{-} \pi^{+} \mathrm{p}$

The properties of the $\rho$ meson and the $\Delta$ baryons are summarized in table 5.5.1.

Our aim is to identify the $\rho^{0}$ vector meson by separating it from the background as much as possible, and finally exploiting the extra informations provided by the vectorial meson. Any contributions of the interference between the $\Delta$ and the $\rho^{0}$ will not be explicitly identified, leaving this as the main topic of a future study based on Partial Wave Analysis technique.

\subsection{Invariant Mass distributions}

The information about the dominant reaction dynamics can be investigated by looking at the three different combinations of plots which correlate the invariant mass distribution of the couple $\pi^{+}-\pi^{-}, I M\left(\pi^{+} \pi^{-}\right)$with the invariant mass distributions from the couple $\pi^{+}-p$ and $\pi^{-}-p, I M\left(\pi^{+} p\right)$ and $I M\left(\pi^{-} p\right)$, respectively. These distributions are plotted after having applied the procedure discussed in chapter 4 to select the final state $p \pi^{+} \pi^{-}$. In Figure 5.8 the correlation plots for the entire photon energy range $\left(0.9 \mathrm{GeV}<E_{\gamma}<2.5 \mathrm{GeV}\right)$ of the gold2 dataset are shown; the different contributions from the $\rho$ vector meson and the $\Delta$ are pointed out by labels and green arrows. The entity of the different contributions can be better estimated by looking at the same correlation plots divided in eight energy bins $200 \mathrm{MeV}$ wide, as shown in Figures 5.9, 5.10 and 5.11. One observes that the reaction $\gamma \mathrm{p} \rightarrow \pi^{+} \pi^{-}$ $\mathrm{p}$ is dominated by the contribution from the $\Delta(1232)^{++}$and $\Delta(1232)^{0}$ for photon energies below $1.5 \mathrm{GeV}$ and by the $\rho$ photoproduction at higher energies.

A first cut that one can apply in order to reject most of the background source from the $\Delta^{+} \pi^{-}$and $\Delta^{-} \pi^{+}$is on the invariant mass distributions $I M\left(\pi^{+} p\right)$ and $I M\left(\pi^{-} p\right)$. The chosen cuts are $I M\left(\pi^{+} p\right)>1.3 \mathrm{GeV}$ and $I M\left(\pi^{-} p\right)>1.3 \mathrm{GeV}$ and their effect on the invariant mass distribution is shown in Figure 5.12. 

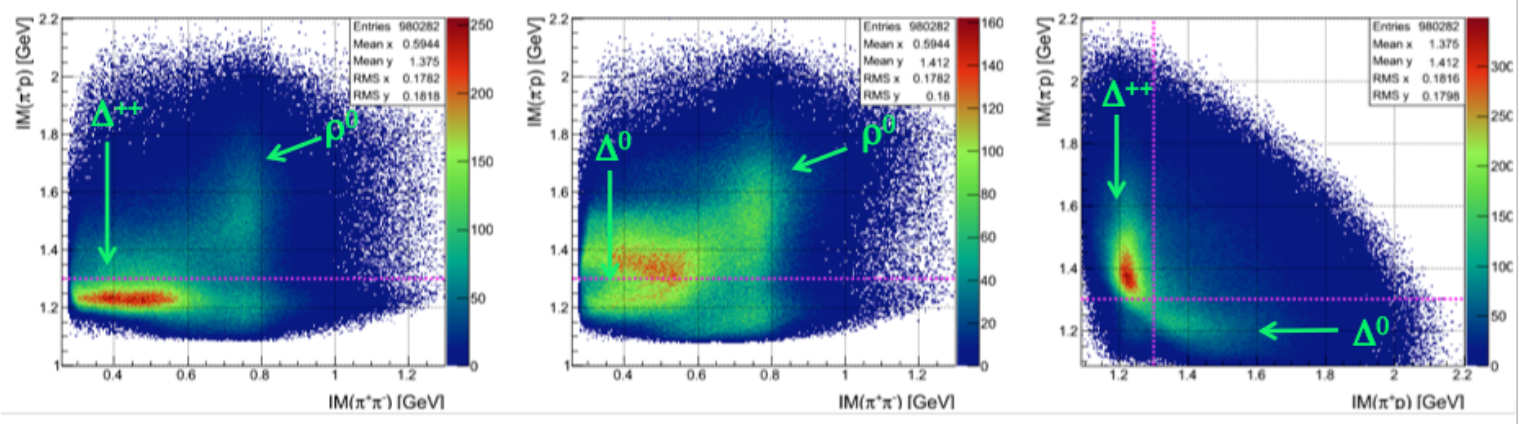

Figure 5.8: From the left: $I M\left(\pi^{+} p\right)$ vs $I M\left(\pi^{+} \pi^{-}\right), I M\left(\pi^{-} p\right)$ vs $I M\left(\pi^{+} \pi^{-}\right)$and $I M\left(\pi^{+} p\right)$ vs $I M\left(\pi^{-} p\right)$ for the entire photon energy range $E_{\gamma} \in[0.9,2.5]$ of the gold2 dataset; the different contributions from the $\Delta$ and the $\rho$ are indicated by the green arrows.

As explained above in section 5.5, the differential cross section for the $\rho$ photoproduction shows an exponential fall-off at small values of the squared recoil momentum $\mathrm{t}$, which implies that this process has more probability to happen at small values of $\mathrm{t}$, or, that is the same, at forward angles. The correlation of the transferred momentum - $t$ with the polar angle of the $\pi^{+} \pi^{-}$pair in the center-of-mass system is shown in Figure 5.13. To further isolate and emphasize the contribution of the $\rho$ photoproduction from the background, a selection cut on the transferred momentum $t$ is applied. The chosen cut is $-\mathrm{t}<0.5 \mathrm{GeV} / \mathrm{c}$, and we can see from table 5.2 that it corresponds to different forward angular ranges in $\theta_{\pi^{+} \pi^{-}}^{C M}$. In Figure 5.14 the transferred momentum -t versus the invariant mass distribution $I M\left(\pi^{+} \pi^{-}\right)$is shown. The cut applied is represented by the dotted magenta lines at $-\mathrm{t}=0.5 \mathrm{GeV}$. The projections corresponding to the selected and rejected events are also shown in the bottom set of plots.

Looking at both Figures 5.13 and 5.14 one can observe that most of the $\rho$ events are actually concentrated in the region selected by imposing the cut on the transferred momentum. The invariant mass distribution $I M\left(\pi^{+} \pi^{-}\right)$plotted after the selection on the transferred momentum is show in Fig. 5.15.

Finally we impose that the incident photon energy must be greater than $1.3 \mathrm{GeV}$; this cut helps to further clean up the invariant mass distribution of the $\pi^{+} \pi^{-}$pair; as one can observe by looking at Figures 5.16 and 5.17, the invariant mass $I M\left(\pi^{+} \pi^{-}\right)$ plotted after having applied the cut on the transferred momentum, only few events remain in the energy region below $E_{\gamma}<1.3 \mathrm{GeV}$ and they mostly come from the background, justifying our cuts rejecting the low photon energy region. 

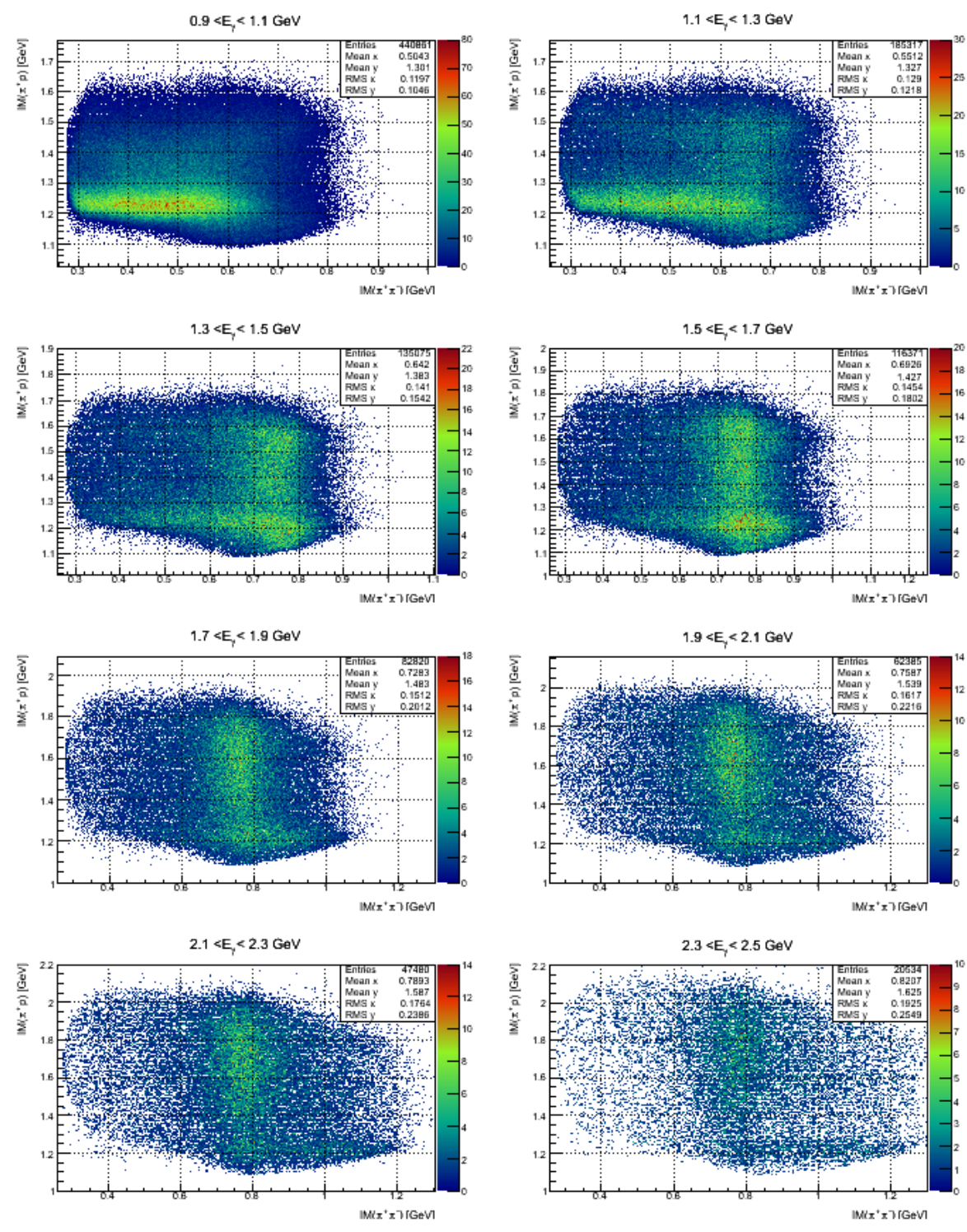

Figure 5.9: $I M\left(\pi^{+} p\right)$ vs $I M\left(\pi^{+} \pi^{-}\right)$for eight photon energy bin $200 \mathrm{MeV}$ wide. 

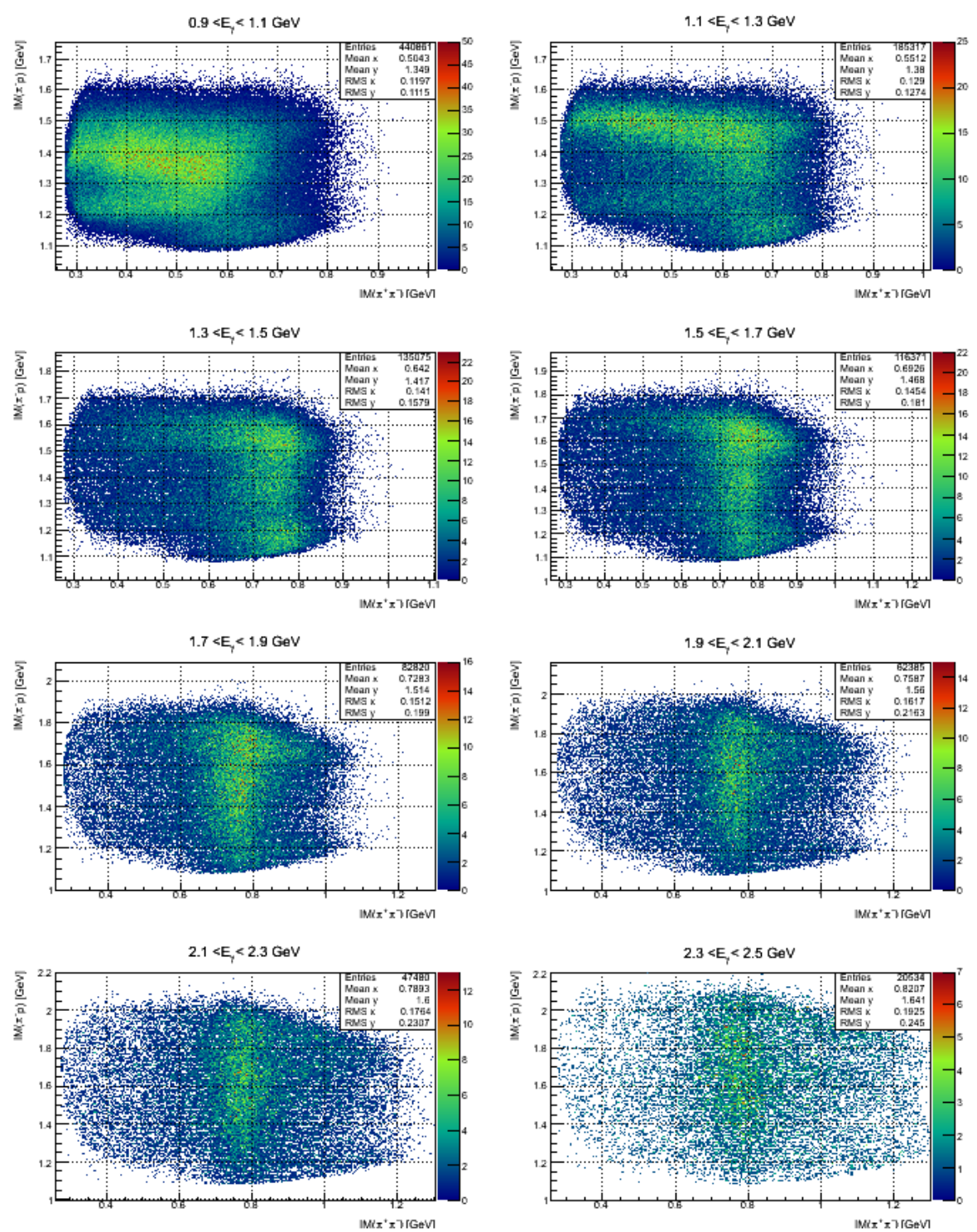

Figure 5.10: $I M\left(\pi^{-} p\right)$ vs $I M\left(\pi^{+} \pi^{-}\right)$for eight photon energy bin $200 \mathrm{MeV}$ wide. 

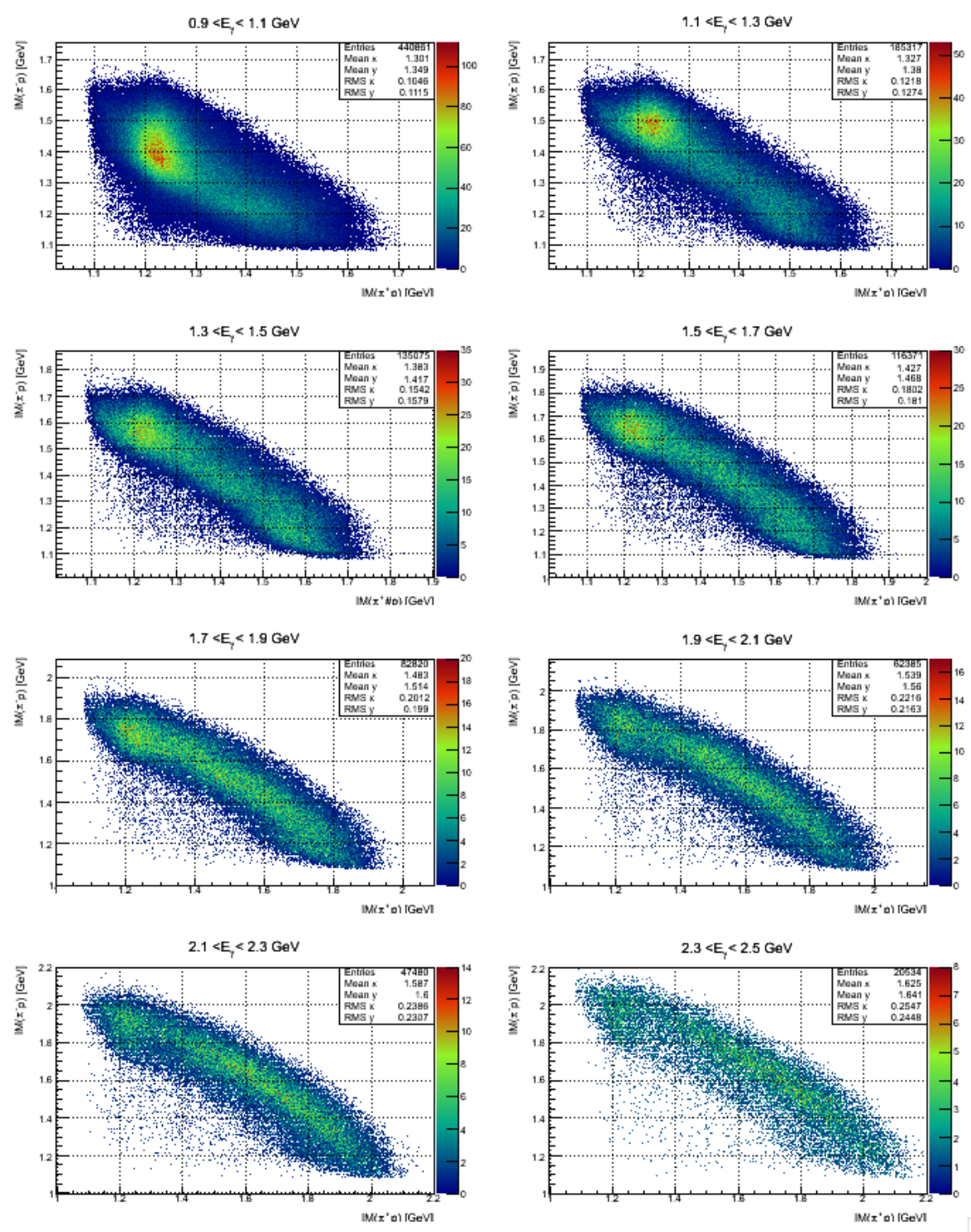

Figure 5.11: $I M\left(\pi^{+} p\right)$ vs $I M\left(\pi^{-} p\right)$ for eight photon energy bin $200 \mathrm{MeV}$ wide. 


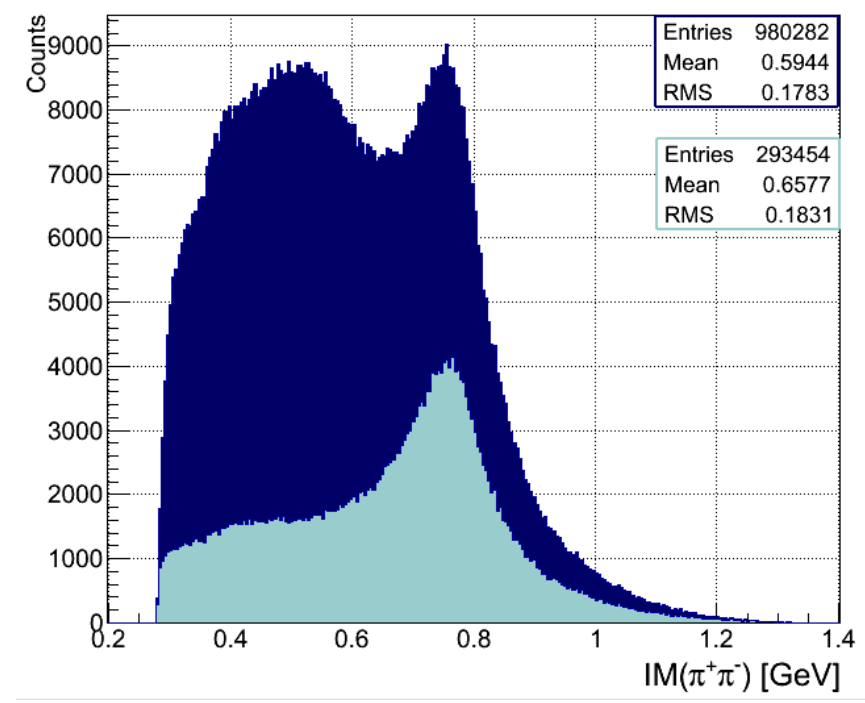

Figure 5.12: Invariant mass distribution $I M\left(\pi^{+} \pi^{-}\right)$; dark blue histogram: $I M\left(\pi^{+} \pi^{-}\right)$after the selection procedure described in chapter 4; cyan histogram (superimposed): $I M\left(\pi^{+} \pi^{-}\right)$after the cut on the invariant mass distributions $I M\left(\pi^{+} p\right)>1.3 \mathrm{GeV}$ and $I M\left(\pi^{-} p\right)>1.3 \mathrm{GeV}$.

\begin{tabular}{|l|l|}
\hline$E_{\gamma}[\mathrm{GeV}]$ & $\theta_{\pi^{+} \pi^{-}}^{C M}[$ degrees $](-t=0.5 \mathrm{GeV})$ \\
\hline 1.4 & 80 \\
\hline 1.5 & 70 \\
\hline 1.6 & 65 \\
\hline 1.7 & 63 \\
\hline 1.8 & 60 \\
\hline 1.9 & 57 \\
\hline 2.0 & 55 \\
\hline 2.1 & 52 \\
\hline 2.2 & 50 \\
\hline 2.3 & 48 \\
\hline 2.4 & 45 \\
\hline
\end{tabular}

Table 5.2: Values of $\theta_{\pi^{+} \pi^{-}}^{C M}$ as a function of the photon energies $E_{\gamma}$ for a fixed values of the transferred momentum $-\mathrm{t}=0.5 \mathrm{GeV}$. 


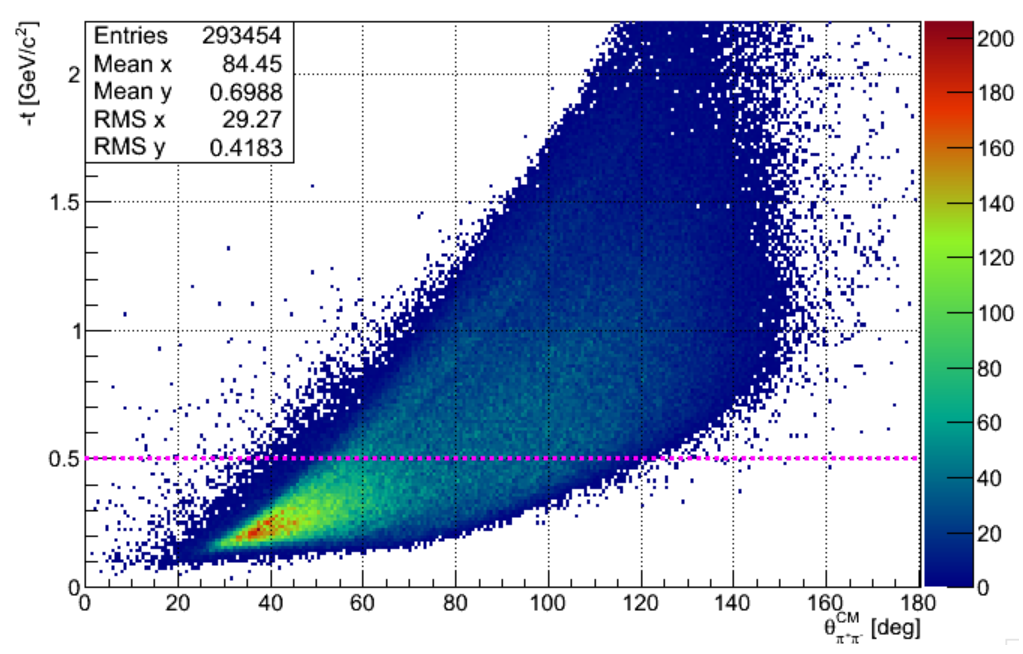

Figure 5.13: The momentum transfer to the proton, -t, versus the polar angle of the pair $\pi^{+}-\pi^{-}$in the center-of-mass frame. The cut imposed $(-\mathrm{t}<0.5 \mathrm{GeV} / \mathrm{c})$ is represented by the magenta dotted line.

The effect of the cuts on $\mathrm{t}$ and $E_{\gamma}$ can be also seen in Figure 5.18 which shows the $\theta_{C M}^{\pi^{+} \pi^{-}}$distribution after different cuts have been applied.

The final invariant mass $I M\left(\pi^{+} \pi^{-}\right)$after all the applied cuts is shown in Figure 5.19. It can be clearly seen that the remaining background is actually small and it is mostly due to non-resonant direct photoproduction of $\pi^{+} \pi^{-}$pairs and residual interference contributions from the $\Delta \pi$ production.

As can be seen in Figure 5.20 this is confirmed by looking at the invariant mass distribution $I M\left(\pi^{+} \pi^{-}\right)$from simulated background data: after applying all selection cuts for the $\rho$ photoproduction, a flat distribution of residual background events remains. 

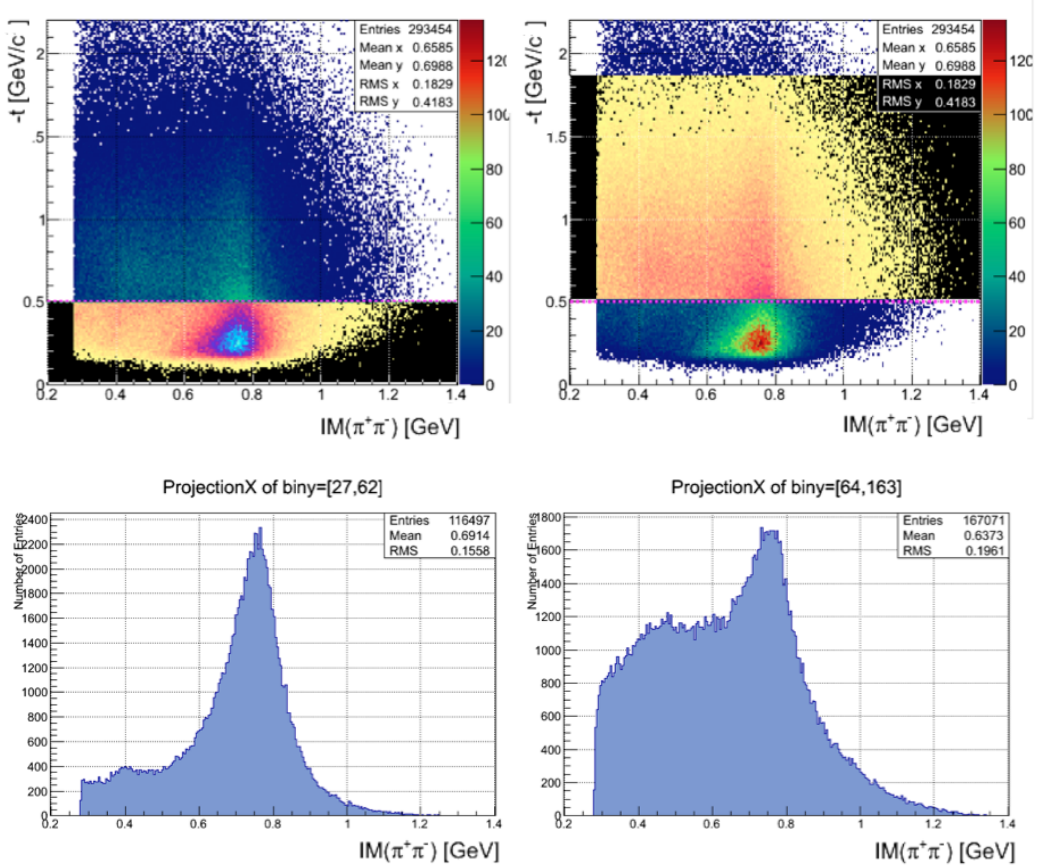

Figure 5.14: Top: transferred momentum -t vs the invariant mass distribution $I M\left(\pi^{+} \pi^{-}\right)$after applied cuts on the $I M\left(\pi^{+} p\right)$ and $I M\left(\pi^{-} p\right)$; the selection applied is represented by the magenta dotted line; highlighted regions correspond to selected $\rho$ events (left) and rejected events (right). Bottom: the projections $I M\left(\pi^{+} \pi^{-}\right)$of the selected and rejected events, respectively, corresponding to the highlighted regions of top plots. 


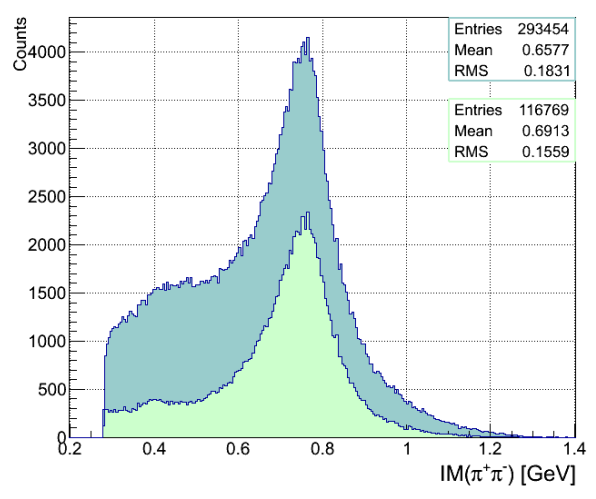

Figure 5.15: Left: the cyan histogram is the invariant mass distribution $I M\left(\pi^{+} \pi^{-}\right)$ obtained by applying the cuts $I M\left(\pi^{+} p\right)>1.3 \mathrm{GeV}$ and $I M\left(\pi^{-} p\right)>1.3 \mathrm{GeV}$; superimposed is the green histogram where a cut on the momentum transferred $t>0.5 \mathrm{GeV} / \mathrm{c}$ is also imposed.
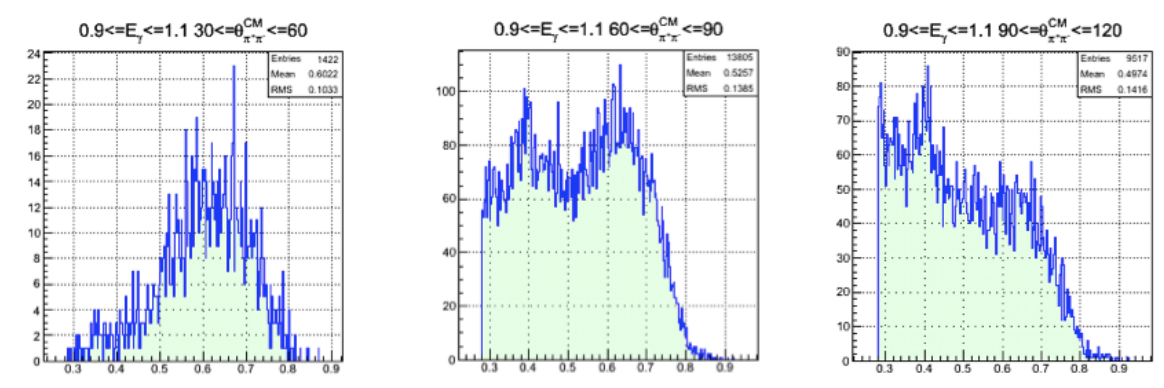

Figure 5.16: Invariant mass distribution $I M\left(\pi^{-} \pi^{-}\right)$for $0.9<=E_{\gamma}<=1.1 \mathrm{GeV}$ in three bins of the polar angle of the $\pi^{+} \pi^{-}$pair in the center-of-mass system $\left(\theta_{+\pi^{-}}^{C M}\right)$ after the cut on the transferred momentum $(\mathrm{t}<-0.5 \mathrm{GeV} / \mathrm{c})$; few events remaining and they mostly come from the background due to $\Delta^{++}$and $\Delta^{0}$. For angles $\theta_{\pi^{+} \pi^{-}}^{C M}<30$ and $\theta_{\pi^{+} \pi^{-}}^{C M}>120$ there are no selected events. 

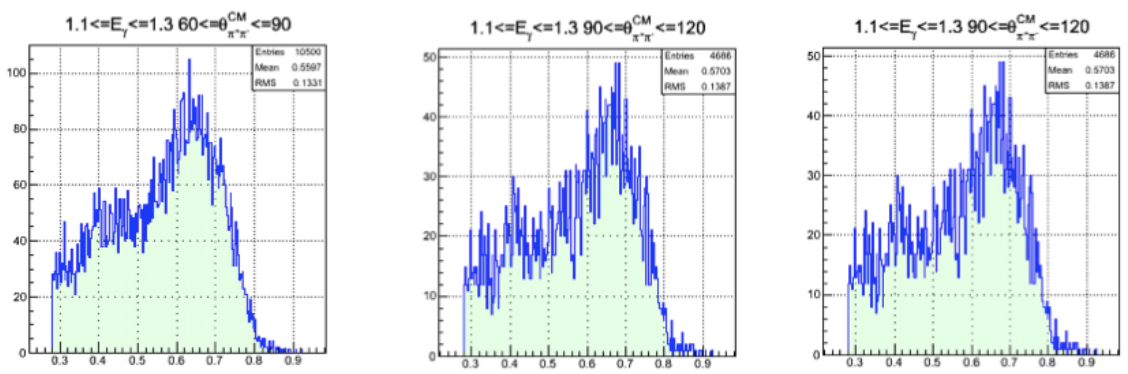

Figure 5.17: Invariant mass distribution $I M\left(\pi^{-} \pi^{-}\right)$for $1.1<=E_{\gamma}<=1.3 \mathrm{GeV}$ in three bins of the polar angle of the $\pi^{+} \pi^{-}$pair in the center-of-mass system $\left(\theta_{+}^{C M}\right)$ after the cut on the transferred momentum $(\mathrm{t}<-0.5 \mathrm{GeV} / \mathrm{c})$; few events remaining and they mostly come from the background due to $\Delta^{++}$and $\Delta^{0}$. For angles $\theta_{\pi^{+} \pi^{-}}^{C M}<30$ and $\theta_{\pi^{+} \pi^{-}}^{C M}>120$ there are no selected events.

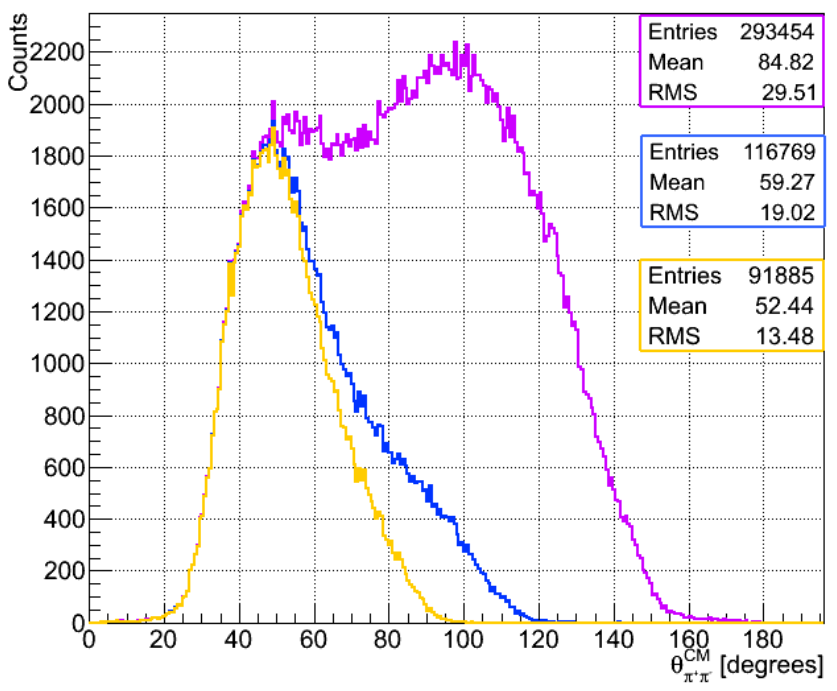

Figure 5.18: $\theta_{C M}^{\pi^{+} \pi^{-}}$after different cuts applied. Violet histogram is after the cut on $I M\left(\pi^{+} p\right)>1.3 \mathrm{GeV}$ and $I M\left(\pi^{-} p\right)>1.3 \mathrm{GeV}$, azure histogram has also the cut $\mathrm{t}>0.5$ and the yellow histogram has $E_{\gamma}>1.3 \mathrm{GeV}$. 


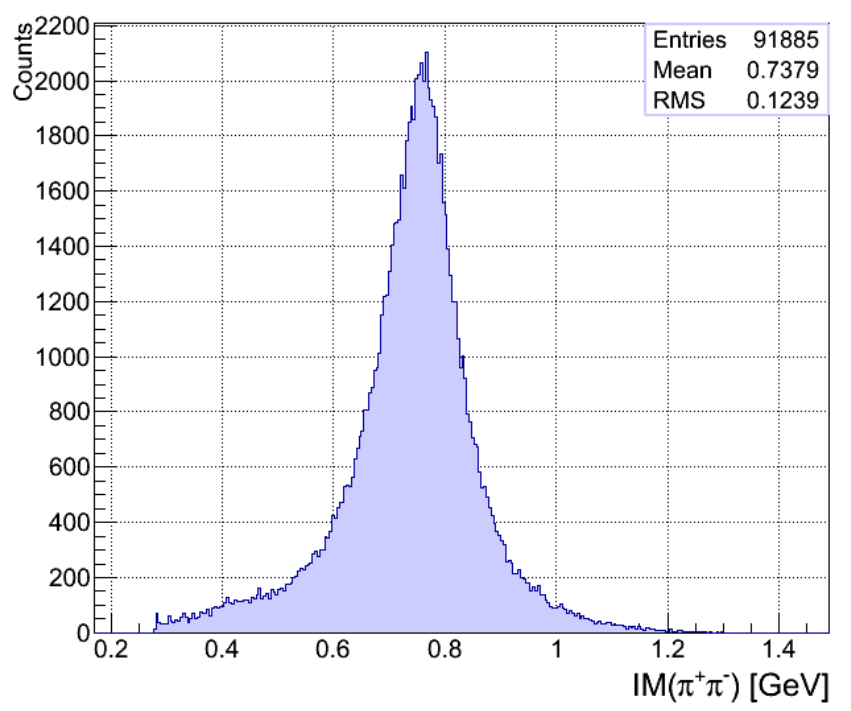

Figure 5.19: The final $I M\left(\pi^{+} \pi^{-}\right)$distribution plotted after the selection procedure. 


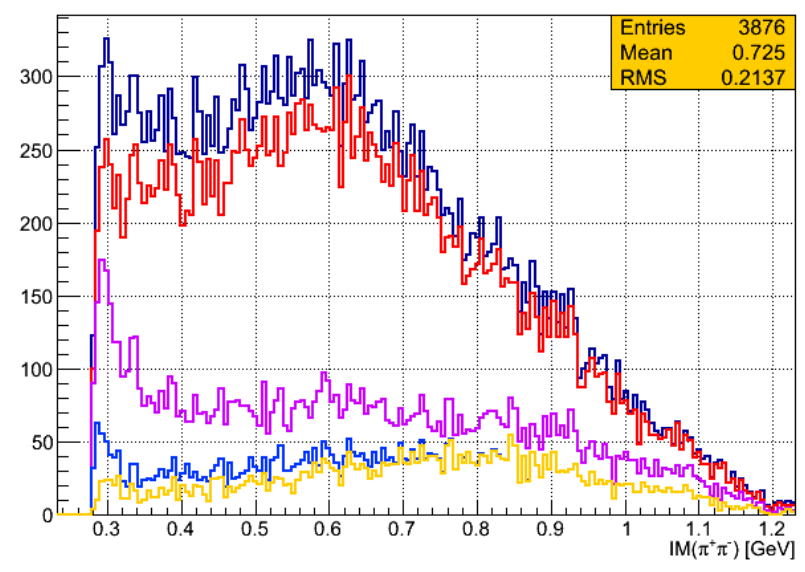

Figure 5.20: Invariant mass distribution $\operatorname{IM}\left(\pi^{+} \pi^{-}\right)$from simulated background data, for different cuts imposed in the analysis. The blue histogram is the $I M\left(\pi^{+} \pi^{-}\right)$ for all background events; the red histogram is the $I M\left(\pi^{+} \pi^{-}\right)$after applying a cut on the $M M\left(\pi^{+} \pi^{-}\right)$closing the $\gamma p \rightarrow \pi^{+} \pi^{-} p$ kinematics; the violet histogram is the $I M\left(\pi^{+} \pi^{-}\right)$after applying a cut on the invariant masses $I M\left(\pi^{+} p\right)>1.3 \mathrm{GeV}$ and $I M\left(\pi^{-} p\right)>1.3 \mathrm{GeV}$; the azure histogram is the $I M\left(\pi^{+} \pi^{-}\right)$after imposing a cut on $\mathrm{t}$, and finally the yellow histogram is the $I M\left(\pi^{+} \pi^{-}\right)$after imposing a cut on $E_{\gamma}>1.3$ $\mathrm{GeV}$. 


\subsection{Background estimation and sPlot method}

One possible approach to disentangle the $\rho$ events from the background contamination is the sPlot technique [69], which basically allows to unfold two statistical distributions such as those of signal and background events. The method is based on the idea that each physical event is characterized by a subset of variables which can be split into two components. The first component is a set of variables whose distributions are assumed to be known and are called discriminating variables; the second components are variables whose distributions are unknown or considered as such, and are called control variables. The sPlot technique allows to reconstruct the distributions for the control variables without any a a priori knowledge on these variables. The idea is to use the knowledge on the discriminating variables distributions, to infer the distributions of the control variables.

An important requirement to unfold the distributions of discriminating and control variables is that they must be independent. In our case the discriminating variable is the proton Missing Mass MM(p), while the control variables are the photon energy $E_{\gamma}$ and the polar angle of the pion pair in the center of mass, $\theta_{\pi^{+} \pi^{-}}^{C M}$ as a function of which the E asymmetry will be extracted. In particular we use a Breit-Wigner curve to model the signal distribution of the $\mathrm{MM}(\mathrm{p})$ (centered around the nominal mass of the $\rho$ vector meson) and a third order Chebycev polynomial for the background, as shown in Figure 5.21.

In this thesis work a preliminary application of the SPlot technique has been implemented. Further studies are necessary to check the systematic effects and the stability of the procedure, therefore it will not be used to extract the E polarization observables in the following. A first encouraging result comes from the fact that, as can be seen from Figure 5.21, the area below the background distribution is less than $5 \%$ of the signal area. We report the preliminary results of the E asymmetry for all selected events, where the residual background is estimated to be of the order of $5 \%$, less than the statistical error. 


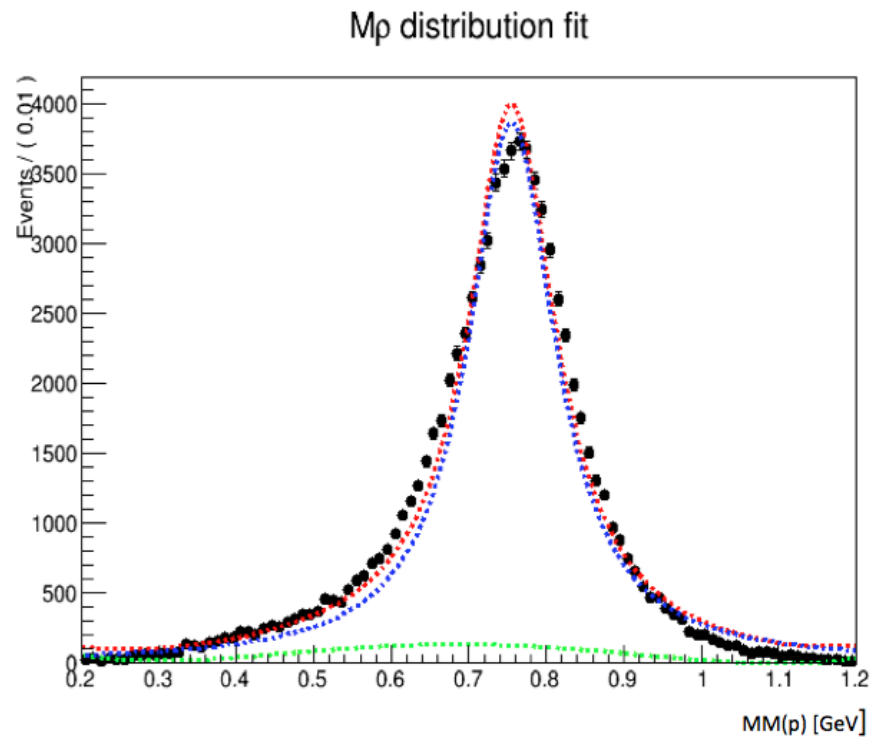

Figure 5.21: $\mathrm{MM}(\mathrm{p})$ distribution fitted through the Sweight technique. The total function (Red histogram) is composed of a Breit-Wigner for the signal (Blue histogram) and a Chebychev third order polynomial for the background (green histogram). Black points are experimental data. 


\subsection{Extraction of the Beam-Target asymmetry (E)}

The cross section for vector meson photoproduction can be written similarly to the pseudoscalar case. For the experimental condition of circularly polarized photons and a longitudinal polarized target, it has the form [70]:

$$
\frac{d \sigma}{d \Omega}=\frac{d \sigma_{0}}{d \Omega}\left(1-\Lambda_{z} \delta_{\odot} E\right)
$$

where we remind that $\sigma_{0}$ denotes the unpolarised cross section, $\delta_{\odot}$ denotes the degree of linear photon polarization, $\delta_{l}$ denotes the degree of circular photon polarization and $\Lambda_{z}$ denotes the longitudinal target polarization. Using the formalism developed in section 5.3, we can write the expression of the double polarization asymmetry E that allows us to extract it from the experimental data:

$$
E=\frac{1}{\delta_{\odot} \Lambda_{z}} \frac{[N(\rightarrow \Leftarrow)-N(\leftarrow \Leftarrow)]}{[N(\rightarrow \Rightarrow)+N(\leftarrow \Rightarrow)]}
$$

in this case only the gold2 dataset was used. 

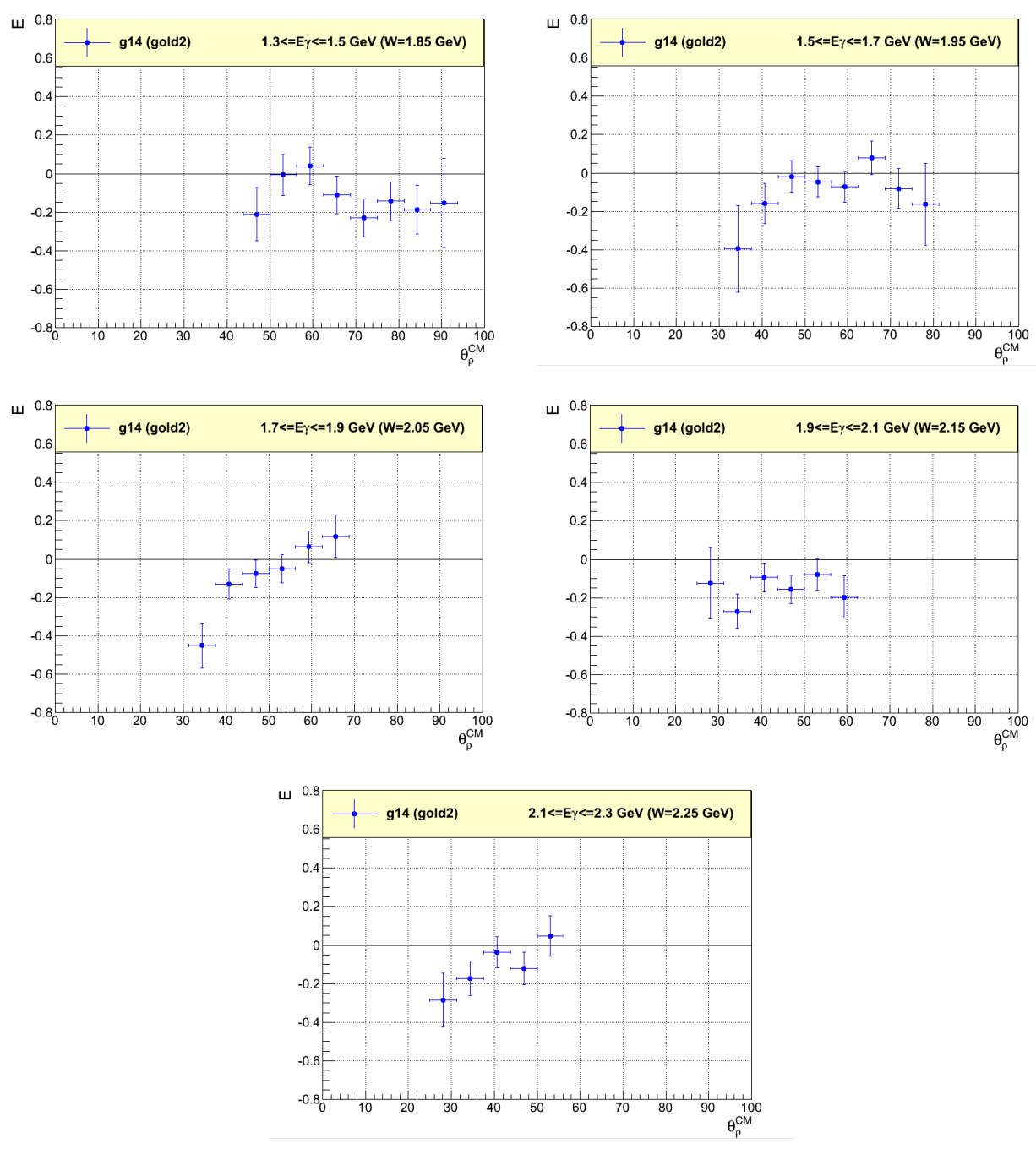

Figure 5.22: Measured Beam-target asymmetry E for the reaction $\gamma p \rightarrow \rho^{0} p$ in the Photon Energy Range $E_{\gamma} \in[1.3,2.3] \mathrm{GeV}$. 


\section{Chapter 6}

\section{Conclusions}

In this thesis work we present the extraction of two of the polarization observables for the double charged pion photoproduction both on proton and neutron target: the beam asymmetry $\left(I^{\odot}\right)$, which arises if the photon beam is circularly polarized, and the helicity difference $P_{z}^{\odot}$, which arises if the photon beam is circularly polarized and the target is longitudinal polarized.

The results of the observable $\left(I^{\odot}\right)$ for the reaction $\gamma p \rightarrow \pi^{+} \pi^{-} p$ is compared with the result from two previous experiments, both performed at the JLab, g1c [71] and [72] and with the model of A.Fix [73]. The observable $I^{\odot}$, extracted from the g14 data, shows the expected odd symmetry and is in overall agreement with the previous experimental works but not with the theoretical model. The observable $I^{\odot}$ extracted from the analysis of the channel $\gamma n \rightarrow \pi^{+} \pi^{-} n$ is also presented. Currently, there are no models and no previous works which compare this measurement to, since these are the first result for the extraction of the observable $I^{\odot}$ off a neutron target in the world.

The measurement of $\mathbf{P}_{\mathbf{z}}{ }^{\odot}$ asymmetry from the double charged pion photoproduction off a proton target is presented as well, and compared with the previous results from the CLAS g9 data [72] and with the model of A.Fix [73]. The very next step will be the evaluation of systematic uncertainties and a systematic check of the symmetry of the extracted observables. The agreement with the results from the g9 experiment is quite good. The agreement with the model is good in trend but not in amplitude. A first approach to disentangle the $\rho$ from the background concurrent reactions $\gamma p \rightarrow \pi^{+} \Delta^{0}, \gamma p \rightarrow \pi^{-} \Delta^{++}$is discussed and the possibility to apply the sPlot technique is investigated. The sPlot technique, along with other probabilistic methods (i.e. Qvalue [74], BDT [75]) for separating signals from backgrounds, will be investigated in deep in a future upcoming study. The first results available on the double polarization beam-target asymmetry $\mathrm{E}$, for the proton target are finally presented. Also in this case, the evaluation of systematic uncertainties will be the object of a future study. 


\section{Appendix A}

\section{Least squares fitting with Lagrange multipliers}

In this section the formalism used in the kinematic fitting using the Lagrange multipliers method will be developed. Let us denote the $\mathrm{m}$ unknown parameters with $\vec{x}$ and the n measured quantities with $\vec{y}$. The actual measured quantities and their errors are the n-vectors $\vec{\eta}$ and $\vec{\epsilon}$, respectively. We can write:

$$
\vec{\eta}=\vec{y}+\vec{\epsilon}
$$

The vectors $\vec{x}$ and $\vec{y}$ are related by the r constraint functions:

$$
f_{k}(\vec{x}, \vec{y})=0 \quad k=1,2, \ldots, r .
$$

The first approximation of the unknows is denoted as $\vec{x}_{0}$. We take $\vec{y}_{0}=\vec{\eta}$ and require the constraint functions to be approximately linear near $\left(\vec{x}_{0}, \vec{y}_{0}\right)$.

It is possible to Taylor expand to first order the constraint functions, as follows:

$$
f_{k}(\vec{x}, \vec{y}) \approx f_{k}\left(\vec{x}_{0}, \vec{y}_{0}\right)+\sum_{i}^{m}\left(\frac{\partial f_{k}}{\partial x_{i}}\right)\left(\vec{x}_{0}, \vec{y}_{0}\right)\left(x^{i}-x_{0}^{i}\right)+\sum_{i}^{n}\left(\frac{\partial f_{k}}{\partial y_{i}}\right)\left(\vec{x}_{0}, \vec{y}_{0}\right)\left(y^{i}-y_{0}^{i}\right)
$$

It is convenient to introduce the following matrices:

$$
a_{i j}=\left(\frac{\partial f_{i}}{\partial x_{j}}\right)\left(\vec{x}_{0}, \vec{y}_{0}\right), \quad A=\left(\begin{array}{cccc}
a_{1,1} & a_{1,2} & \cdots & a_{1, m} \\
a_{2,1} & a_{2,2} & \cdots & a_{2, m} \\
\vdots & \vdots & \ddots & \vdots \\
a_{r, 1} & a_{r, 2} & \cdots & a_{r, m}
\end{array}\right)
$$




$$
b_{i j}=\left(\frac{\partial f_{i}}{\partial y_{j}}\right)\left(\vec{x}_{0}, \vec{y}_{0}\right), \quad B=\left(\begin{array}{cccc}
b_{1,1} & b_{1,2} & \cdots & b_{1, m} \\
b_{2,1} & b_{2,2} & \cdots & b_{2, m} \\
\vdots & \vdots & \ddots & \vdots \\
b_{r, 1} & b_{r, 2} & \cdots & b_{r, n}
\end{array}\right)
$$

the vector of constraints equations:

$$
\vec{c}=\left(\begin{array}{c}
f_{1}\left(\vec{x}_{0}, \vec{y}_{0}\right) \\
f_{2}\left(\vec{x}_{0}, \vec{y}_{0}\right) \\
\vdots \\
f_{r}\left(\vec{x}_{0}, \vec{y}_{0}\right)
\end{array}\right)
$$

and the differential vectors:

$$
\begin{aligned}
& \vec{\xi}=\vec{x}-\vec{x}_{0} \\
& \vec{\delta}=\vec{y}-\vec{y}_{0}
\end{aligned}
$$

We can rewrite equation (A.3) as follows:

$$
A \vec{\xi}+B \vec{\delta}+\vec{c}=0
$$

The minimization quantity is given by:

$$
\vec{\delta}^{T} C_{\eta}^{-1} \vec{\delta}
$$

where $C_{\eta}$ is the covariance matrix of the measured quantities. In the particular case of independent measurements, the equation (A.10) takes the more familiar form:

$$
\sum_{j=1}^{n} \frac{\epsilon_{j}^{2}}{\sigma_{j}^{2}}
$$

We introduce the Lagrangian:

$$
L=\vec{\delta}^{T} C_{\eta}^{-1} \vec{\delta}+2 \vec{\mu}^{T}(A \vec{\xi}+B \vec{\delta}+\vec{c})
$$

- where $\vec{\mu}$ is the r-vector of the Lagrangian multipliers. Using the fact that $\vec{\delta}$ and $\vec{\xi}$ are independent variables, setting the total differential to zero yields:

$$
\frac{\partial L}{\partial \delta_{i}}=\frac{\partial L}{\partial \xi_{i}}=0
$$

Substituting the solution to equations (A.13) into (A.9) gives:

$$
\vec{\xi}=-\left(A^{T} C_{B} A\right)^{-1} A^{T} C_{B} \vec{c}
$$




$$
\vec{\delta}=-C_{\eta} B^{T} C_{B}(\vec{c}-A \vec{\xi})
$$

where $C_{B}=\left(B C_{\eta} B^{T}\right)^{-1}$. The least squares estimates for the parameters $\vec{x}$ and improved measurement $\vec{y}$ are then:

$$
\begin{aligned}
& \vec{x}=\vec{x}_{0}+\vec{\xi} \\
& \vec{y}=\vec{y}_{0}+\vec{\delta}
\end{aligned}
$$

When the constrain functions are not linear the process should be iterated. The estimates of the measurement errors are the n-vector:

$$
\vec{\epsilon}=\vec{\eta}-\vec{y}
$$

where $\vec{y}$ are the improved measurements obtained from the final iteration and $\vec{\eta}$ are the measured values.

If the errors are normally distributed and the constraints function are sufficiently linear near $\left(\vec{x}_{0}, \vec{y}_{0}\right)$, then $\vec{\epsilon}^{T} C_{\eta}^{-1} \vec{\epsilon}$ follow a $\chi^{2}$ distribution with r-m degrees of freedom. We can use propagation of errors to write the covariance matrix for the improved measurement as:

$$
C_{y}=C_{\eta}-C_{\eta} B^{T} C_{B} B C_{\eta}+C_{\eta} B^{T} C_{B} A\left(A^{T} C_{B} A\right)^{-1} A^{T} C_{B} B C_{\eta}
$$

and the covariance matrix for the parameters as:

$$
C_{x}=\left(A^{T} C_{B} A\right)^{-1}
$$




\section{Bibliography}

[1] W. E. Burcham, M. Jobes "Nuclear and Particle Physics" Longman Scientific Technical

[2] Johan Messchendorp for the BESIII Collaboration "Physics with Charmonium - A few recent highlights" http://arxiv.org/pdf/1306.6611.pdf

[3] M. S. Bhagwat, M. A. Pichowsky, C. D. Roberts and P. C. Tandy, "Analysis of a quenched lattice-QCD dressed-quark propagator" Phys. Rev. C 68, 015203 (2003)

[4] M. S. Bhagwat and P. C. tandy, "Analysis of full-QCD and quenched-QCD lattice propagators" aip conf. proc. 842, 225-227 (2006).

[5] P. O. Bowman et al., "Unquenched quark propagator in Landau gauge" Phys. Rev. D 71, 054507 (2005)

[6] "The history of QCD", CERN COURIER, Sept 27, 2012

[7] J. Beringer et al. [Particle Data Group Collaboration] Phys. ReV. D 86, 010001 (2012)

[8] K. Nakamura et al. [Particle Data Group Collaboration] J. Phys. GG 37, 075021 (2010)

[9] R. Gupta "Introduction to lattice QCD" http://arxiv.org/pdf/heplat/9807028.pdf

[10] http://pdg.lbl.gov/2012/reviews/rpp2012-rev-lattice-qcd.pdf

[11] Robert G. Edwards et al. "Excited state baryon spectroscopy from lattice QCD" Phys. Rev. D 84 (2011) 074508

[12] Lin, Huey-Wen "Review pf Baryon Spectroscopy in Lattice QCD" Chin.J.Phys. 49 (2011) 827

[13] E. Santopinto "Interacting quark-diquark model of baryons" Phys. Rev. C 72 $022201(2005)$ 
[14] R. Koniuk, N. Isgur "Where Have All the Resonances Gone? An Analysis of Baryon Couplings in a Quark Model with Chromodynamics" Phys. Rev. Lett. 44, 845 (1980)

[15] S. Hawking "A brief history of time"

[16] H. Huneke et al. BONN-IR-80-24 (1980)

[17] I. Arai, H.Fujii Nucl. Phys B194, 251

[18] K.Fujii et al. Nucl. Phys B197, 365

[19] R. Kajikawa: "Pion Photoproduction and Compton Scattering in the Resonance Region" International Symposium o Lepton and Photon Interactopm at High Energies, Bonn (1981)

[20] B. Krusche "Photoproduction of Meson off Nuclei - The Photonuclear Programs at ELSA and MAMI" Journal of Physics: Conference Series 239 (2012) 012003

[21] W. Roberts and T. Oed "Polarization observables for two-pion production off the nucleon" Phys. Rev. C71, 055201 (2005)

[22] W.-T. Chiang, and F. Tabakin, "Completeness rules for spin observables in pseudoscalar meson photoproduction" Phys. Rev. C 55, 2054 (1997).

[23] I.S. Barker, A. Donnachie and J.K. Storrow "Complete experiments in pseudoscalar photoproduction" Nuclear Physics B95 (1975) 347-356

[24] M. Pichowsky, C. Savkly and F. Tabakin "Polarization observables in vector meson photoproduction" Phys. Rev. C 531996

[25] R.A. Arndt, W.J. Briscoe, I.I Strakovsky and R.L. Workman Phys. Rev. C72, $058203(2005)$

[26] H. Dutz et al., Phys. Rev. Lett. 94, 162001 (2005)

[27] H. Dutz et al., Phys. Rev. Lett. 93, 032003 (2004)

[28] F.J. Klein, A.M. Sandorfi et al. " $N^{*}$ resonances in pseudoscalar-meson photoproduction from polarized neutrons in $\vec{H} \vec{D}$ and a complete determination of the $\gamma n \rightarrow K^{0} \Lambda$ amplitude" Jefferson Lab experiment proposal E06-101/g14.

[29] N. Bianchi et al., "Total hadronic photoabsorption cross section on nuclei in the nucleon resonance region" Phys. Rev. C 54 (1996) 1688

[30] http://www.jlab.org/photos. 
[31] H. Olsen and L.C Maximon "Photon and Electron Polarization in High-Energy Bremsstrahlung and Pair Production with Screening", Phys. Rev. 114, 887 (1957)

[32] M. M. Shukla and N.T. Padial "A Calculation of The Debye Characteristic Temperature of Cubic Crystals" Revista Brasileira de Fisica 3, 1 (1973)

[33] B. Mukherjee and A.J.F. Boyle, "On The Debye Characteristic Temperature of Diamond", Phys. Stat. Solidi 22, K131 (1967)

[34] F.H. Dyson "Anisotropy of Bremsstrahlung and Pair Production in Single Crystals" Phys. Rev. 99, 604 (1955)

[35] K. Livingston, Nucl. Instr. and Meth. A 603, 205 (2009).

[36] D. Marchand, A. Natter and R. Van de Vyver, "A High Energy, HighIntensity ad Highly Polarized Photon beam for ELFE@CERN using Coherent Bremsstrahlung" http : //www.ichdenkmal.de/hpan/singledocs/hapheep realrep - marchand + natter.pdf

[37] D.I. Sober et al. Nucl. Instrum. and Methods in Phys. research A 440 263-284 (2000)

[38] B.A. Mecking et al. Nucl. Instrum. and Methods in Phys. research A 503 513$553(2003)$

[39] J. O'Meara et al., "A Superconducting Toroidal Magnet for the CEBAF Large Acceptance Spectrometer", IEEE Trans. Mag. 25, 1902 (1989).

[40] S. Taylor et al., "The CLAS Start Counter", Nucl. Instr. Meth. A 462, 484 (2001).

[41] M.D. Mestayer D.S. Carman and the CLAS coll. "The CLAS Drift Chamber system" ,Nucl. Instr. Meth. A 449 (2000) 81-111

[42] E.S. Smith et al., "The time-of-flight system for CLAS", Nucl. Instr. Meth. A 432,265 (1999).

[43] M. Amarian et al., "The CLAS forward electromagnetic calorimeter" Nucl. Instr. and Meth. A 460, 239 (2001).

[44] A. Honig "Highly spin-polarized proton samples-large, accessible, and simply produced" Phys. Rev. Lett 19, 1009-10010 (1967)

[45] D.G. Crabb, W. Meyer "Solid polarized targets for nuclear and particle physics experiments" Annu. Rev. Nucl. Part. Sci. 47:67-109 (1997) 
[46] C.D. Bass et al. "A portable cryostat for the cold transfer of polarized solid HD targets: HDice" Nucl. Instrum. and Methods in Phys. research A 737(0): 107-116 (2014)

[47] V. Laine "Polarimetry of the polarized hydrogen deuteride HDice target under an electron beam" $\mathrm{PhD}$ theses of Vivien Laine, University Blaise Pascal

[48] A. D'Angelo et al. "HD gas distillation and analysis for HD frozen spin target" PoS (PSTP 2013) m07

[49] C.S. Whisnant et al. "HD gas purification for polarized HDice target production at Jefferson Lab" PoS (PSTP 2009) m07

[50] C. Steven Whisnant, Patrick A. Hansen and Travis D. Kelly "Measuring the Relative Concentration of H2 and D2 In HD Gas with Gas Chromatography"', Rev. Sci. Inst. 82, 024101 (2011).

[51] J. Michael Hollas "Modern Spectroscopy" Ed. Wiley John Wiley Sons, Ltd, Fourth Edition

[52] K. Okuno, T. Uda, S. O'Hira, Y. Naruse Journal of Nuclear Science and Technology 28 509-516 (1991)

[53] W.N. Hardy and J.R. Graines "Nuclear Spin Relaxation in Solid HD with H2 Impurity" Phys. Rev. Lett 17, 1278 (1966)

[54] M. Bloom Physica 23, 767 (1957).

[55] A. Honig, Q.Fan X. Wei A. M. Sandorfi and C. S. Whisnant "New investigations of polarized solid HD targets" Nucl. Instrum. and Methods in Phys. Research A 356, 39-46 (1995)

[56] C. Thorn, A. Caracappa AIP Conference Proceedings, 980397 (2008)

[57] M. Bellis, V. Crede, S.Strauch et al. "Measurement of $\pi^{+} \pi^{-}$photoproduction in double-polarization experiment using $C L A S^{\prime \prime}$ Jefferson Lab experiment proposal E06-013.

[58] D. Cords et al. "CLAS Event Format with BOS", CLAS NOTE 94-012, 1994

[59] V. Bobel et al. "The BOS System for CLAS Detector", Unpublished, 1995

[60] R. Brun et al., "ROOT Data Analysis Framework", http://root.cern.ch/drupal/

[61] K. Livingstone, Bank Event Extraction Routines http://nuclear.gla.ac.uk/ kl/rootbeer/ 
[62] A. S. Iljinov et al., "Extension of the intranuclear cascade model for photonuclear reactions at energies up to 10-GeV" Nucl. Phys. A 616, 575 (1997)

[63] E. Pasyuk "Brief User Guide to GPID" CLAS NOTE 2007-008

[64] E. Pasyuk "Energy loss corrections for charged particles in CLAS" CLAS NOTE 2007-016

[65] M. Williams, D. Applegate and C.A. Meyer "Determining Momentum and Energy Corrections for g1c Using Kinematic Fitting" CLAS NOTE 94-012

[66] D. Keller "Techniques in Kinematic Fitting" CLAS NOTE 2010 - 015

[67] M. Williams "Measurement of Differential Cross Sections and Spin Density Matrix Elements along with a Partial Wave Analysis for $\gamma p \rightarrow p \omega$ using CLAS at Jefferson Lab" Jlab Thesis

[68] The SAPHIR Collaboration (C. Wu et al.) Eur. Phys. J. A 23 (2) (2005)

[69] M. Pivk "sPlot: a quick introduction" http://arxiv.org/pdf/physics/0602023.pdf

[70] H. Eberhardt "Measurement of polarization observables in omegaphotoproduction" AIP Conf.Proc. 1432 (2012) 413-416 arXiv:1107.3966 [nucl-ex]

[71] S.Strauch, B.I Berman and the CLAS collaboration "Beam-Helicity Asymmetries in Double-Charged-Pion Photoproduction on the Proton" Phys. ReV. Lett 95 162003-1-16203-5 (2005)

[72] S. Park et al. "Polarization observables in $\gamma p \rightarrow p \pi^{+} \pi^{-}$using the $g 9$ a (FROST) target and CLAS spectrometer", CLAS NOTE 2013-012

[73] A. Fix et al. "Double pion photoproduction on nucleon and deuteron" Eur. Phys. J. A 25 (2005) 115

[74] M. Williams, M. Bellis, C. Meyer "Separating Signals from Non-Interfering Background using Probabilistic Event Weightings" http://arxiv.org/pdf/0804.3382.pdf

[75] C. Meyer "Boosted Decision Trees, a powerful event classifiers" http://www.curtismeyer.com/articles/bkgrnd/phystat05-proc.pdf 